\title{
The evolution of jaw mechanism and dental function in heterodont crocodyliforms
}

\author{
ATTILA ÖSI ${ }^{1}$ \\ ${ }^{1}$ Hungarian Academy of Sciences - Eötvös Loránd University, Lendület Dinosaur Research \\ Group, Pázmány Péter sétány 1/c, Budapest, 1117, Hungary; e-mail: hungaros@gmail.com
}

\begin{abstract}
Heterodont dentition sometimes including multicuspid crowns appeared in numerous fossil forms through all main lineages of the Crocodyliformes. Teeth in these complex dentitions frequently bear wear facets that are exclusive indicators of tooth-tooth occlusion. Besides dental features, specializations of the jaw apparatus, jaw adductors and mandibular movement can be recognized, all reflecting a high variability of jaw mechanism and of intraoral food processing. Comparative study of these features revealed four main types of jaw mechanism some of which evolved independently in several lineages of Crocodyliformes. Isognathous orthal jaw closure (precise jaw joint, rough wear facets) is characteristic for heterodont protosuchians and all forms possessing crushing posterior teeth. Proal movement (protractive powerstroke) occured independently in Malawisuchus and Chimaerasuchus is supported by the antagonistic, vertically oriented carinae. Developed external adductors are the main indicators of palinal movement (retractive powerstroke) that evolved at least two times in various South American taxa. The fourth type (in Iharkutosuchus) is characterized by lateromedial mandibular rotation supported by extensive horizontal wear facets. This evolutionary scenario resembles that of the masticatory system of mammals and suggests that the ecological roles of some mammalian groups in North America and Asia were occupied in Western Gondwana by highly specialized crocodyliforms.
\end{abstract}




\section{INTRODUCTION}

Generally, the feeding apparatus and feeding mechanism of crocodyliforms is regarded as conservative because it does not show radical changes during the evolution of the group. Among the few hundred valid species from protosuchians to the 23 extant taxa, tooth and jaw morphology, jaw articulation and adductor muscle architecture did not change significantly in most species. Their dentition is formed usually of widely-spaced, conical, monocusped teeth (although with highly variable in tooth count), dominantly with unserrated mesiodistal carinae mesiodistally. Jaw articulation is precise with quadrate condyles separated by a marked intercondylar groove and the condyles nicely fit into the glenoid cavity of the articular. This is a key feature (along with the wide pterygoid flanges preventing lateromedial movement) to ensure precise orthal movement during opening and closure of the jaws. Of the jaw adductor musculature, the main muscle groups are the pterygoid muscles (MPT) and the external adductors (parts of the MAME). Their state of development and importance in the jaw closure, however, alternate depending on skull architecture and the mode of prey capture. For example, in longirostrine forms (thalattosuchians, dyrosaurs, gavial) being dominantly aquatic piscivorous predators, the bundles of the external adductors responsible for rapid jaw closure (like the temporalis muscles in mammals, Smith 1993) are highly developed and play an extremely important role in food capture. These muscles have short bundles and are responsible for a relatively fast jaw closure (Iordansky 1964, Mueller-Töwe 2006). In small bodied, brevirostrine forms (e.g. Bernissartia, Allognathosuchus, Brachychampsa, Iharkutosuchus) often possessing bulbous crushing teeth the pterygoid muscles responsible for relatively slow but effective jaw closure (like the masseter and pterygoid muscles in mammals, Smith 1993) are the dominant adductors (Ösi and Weishampel 2009, Figure 1). 
In the last decades, however, new discoveries, especially in former Gondwanan landmasses, provided evidence for diverse and abundant assemblages of highly specialized crocodyliforms (Figure 2) that possess heterodont, sometimes mammal-like dentition (Figure 3) (e.g. Clark et al. 1989, Carvalho 1994, Buckley et al. 2000, Pol 2003, Nobre and Carvalho 2006, Marinho and Carvalho 2009, O’Connor et al. 2010, Iori and Carvalho 2011). These forms are usually characterized by complex jaw mechanism complemented by dental occlusion that refers to efficient oral food processing (Ösi 2010). The appearence of these fundamental craniodental features among Cretaceous Gondwanan notosuchians suggests that these small bodied forms „probably filled niches and inhabited ecomorphospace that were otherwise occupied by mammals on northern continents" (O' Connor et al. 2010:748). Nevertheless, crocodyliforms with mammal-like craniodental features were not restricted to the notosuchian clade and to the Cretaceous Gondwana but they also occured in the Early Jurassic of North America and in the Cretaceous of Europe and eastern Asia, where they were represented by both the protosuchian and modern, eusuchian clades (Young 1973, Li 1985, Clark and Fastovsky 1986, Sues et al. 1994, Wu et al. 1995, Pol et al. 2004, Ösi et al. 2007). In addition to these forms with multicusped teeth, the bulbous, crushing teeth in several lineages of the neosuchian crocodyliforms (e.g. Weitzel 1935, Buffetaut and Ford 1979, Buscalioni and Sanz 1990, Brinkmann 1992, Norell et al. 1994, Brochu 1999, Lucas and Estep 2000, Ösi 2010, Ősi and Barrett 2011) represent a further adaptation among the specialized feeding strategies of the group that, again, clearly emphasizes that feeding adaptations within the Crocodyliformes were much more diverse than previously thought.

The aim of this work is to collect, review and compare all these specialized heterodont crocodyliforms in a functional perspective completed with the descripton and detailed illustration of the cranial material and craniodental features. Reconstruction of the main cranial adductors and analyses of dental wear patterns are fulfilled to understand the process 
of dental occlusion and movements of the lower jaw during food processing in each taxon. Putting the taxa in a phylogenetic context, the evolution of craniodental characters and jaw mechanisms is discussed. Results of these investigations offer new insights into the evolution of crocodyliform trophic adaptations and in the case of Gondwanan notosuchians they help to reconstruct possible scenarios of competition, dietary overlap and partitioning between small bodied, heterodont crocodyliforms and mammals.

Institutional Abbreviations - ACAP Association Culturelle, Archéologique et Paléontologique de l'Ouest Biterrois, Cruzy, Hérault, France; BSM, Bayerische Staatssammlung für Paläontologie und Geologie, Münich, Germany; FMNH, The Field Museum, Chicago, USA; HLMD, Hessisches Landmuseum; Darmstadt, Germany; IPFUB, Institut für Paläontologie der Freien Universität, Berlin, Berlin, Germany; IRSNB, Institut Royal des Sciences Naturelles de Belgique, Bruxelles, Belgium; IVPP, Institute of Vertebrate Paleontology and Paleoanthropology, Beijing, People's Republic of China; MACN, Museo Argentino de Ciencias Naturales, Buenos Aires, Argentina; MAL, Malawi Department of Antiqities, Malawi; MCSNT, Museo Civico di Storia Naturale di Trieste, Italy; MN, Museu Nacional, Rio de Janeiro, Brasil; MNHN, Musée National d'Histoire Naturelle, Paris, France; MPMA, Museu de Paleontologia de Monte de Alto, Monte Alto, Brasil; MTM, Hungarian Natural History Museum, Budapest, Hungary; MZSP-PV, Museu de Zoologia, Universidade de São Paulo, Brazil; NHM, Natural History Museum, London, England; UA, University of Antananarivo, Antananarivo, Madagascar; UCMP, Museum of Paleontology, University of California, Berkeley, USA; UFRJ, Universidad Federal do Rio de Janeiro, Brazil. 
Anatomical abbreviations used in the figures:

$\begin{array}{ll}\text { a } & \text { anterior } \\ \text { acav } & \text { accessory cavities of the antorbital } \\ & \text { fossa } \\ \text { acc } & \text { accessory cusp } \\ \text { adt } & \text { anterior dentary tooth } \\ \text { aexa } & \text { attachment area of external } \\ & \text { adductors } \\ \text { agr } & \text { apical groove } \\ \text { aMAMP attachment surface for } \\ & \quad \text { MAMP } \\ \text { aMPTV } & \quad \text { attachment surface for } \\ \text { alv } & \text { alveolus } \\ \text { amx1 } & \text { first maxillary alveolus } \\ \text { an } & \text { angular } \\ \text { aof } & \text { antorbital fenestra } \\ \text { ap } & \text { apical } \\ \text { ar } & \text { articular } \\ \text { awf } & \text { apical wear facet } \\ \text { ba } & \text { basal } \\ \text { bc } & \text { broken crown } \\ \text { bit } & \text { bicusped teeth } \\ \text { bo } & \text { basioccipital } \\ \text { c } & \text { carina } \\ \text { ca } & \text { caniniform tooth } \\ \text { car } & \text { cervical armour } \\ \text { ced } & \text { distal carina of the central cusp } \\ \text { ch } & \text { choana } \\ \text { ci } & \text { cingulum } \\ \text { co } & \text { coronoid } \\ \text { coa } & \text { conical apex } \\ \text { cr } & \text { crest } \\ \text { crc } & \text { central row of cusps } \\ \text { d } & \text { dentale } \\ \text { de } & \text { dentine } \\ \text { d1-10 first to tenth dentary teeth } \\ \text { di } & \text { distal } \\ \text { do } & \text { dorsal } \\ \text { ec } & \text { ectopterygoid } \\ \text { edi } & \text { enamel-dentine interface } \\ \text { emf } & \text { external mandibular fenestra } \\ \text { ena } & \text { external nares } \\ \text { en } & \text { enamel } \\ \text { fm } & \text { foramen magnum } \\ \text { foramen } \\ & \end{array}$

icgr intercuspidate groove

if incisive foramen

imf caudal intermandibular foramen

j jugal

1 lateral

la labial

lacr lacrimal

lac labial cusp

lal last alveolus

larc labial row of cusps

lawf labial wear facet

lca lower caniniform tooth

lco lateral cotyle

ler longitudinal enamel ridges

li lingual

lic lingual cusp

lirc lingual row of cusps

liwf lingual wear facet

ls laterosphenoid

ltf lateral temporal fenestra

m medial

MAMEM Musculus adductor mandibulae externus medialis

MAMEP Musculus adductor mandibulae externus profundus

MAMES Musculus adductor mandibulae externus superficialis

MAMP Musculus adductor mandibulae posterior

mc main cusp

mcq medial condyle of the quadrate

me mesial

MI Musculus intramandibularis

mot molariform teeth

mpf maxilla-palatine foramen

MPSS Musculus pseudotemporalis superficialis

MPTD Musculus pterygoideus dorsalis

MPTV Musculus pterygoideus ventralis

mri median ridge

mut multicusped tooth

$\mathrm{mx}$ maxilla

mx1-10 first to sixth maxillary teeth 


$\begin{array}{ll}\text { mxsh } & \text { maxillary shelf } \\ \text { mxt } & \text { maxillary tooth } \\ \text { na } & \text { nasal } \\ \text { nlca } & \text { notch for the lower caniniform } \\ & \text { tooth } \\ \text { o } & \text { orbit } \\ \text { oc } & \text { occipital condyle } \\ \text { oct } & \text { occlusion of crushing teeth } \\ \text { oMAMEP origin surface for MAMEP } \\ \text { oMAMP origin surface for MAMP } \\ \text { oMPTD origin surface for MPTD } \\ \text { oMPTV origin surface for MPTV } \\ \text { osc } & \text { oblique scratches } \\ \text { ost } & \text { osteoderm } \\ \text { p } & \text { posterior } \\ \text { pa } & \text { parietal } \\ \text { pct } & \text { posterior crushing tooth } \\ \text { pet } & \text { penultimate tooth } \\ \text { pi } & \text { pit } \\ \text { pl } & \text { palatine } \\ \text { pmx } & \text { premaxilla } \\ \text { pmxt } & \text { premaxillary teeth } \\ \text { pm2 } & \text { second premaxillary tooth } \\ \text { ppm } & \text { posterior process of maxilla } \\ \text { pppt } & \text { posterior process of pterygoid } \\ \text { prf } & \text { prefrontal } \\ \text { po } & \text { postorbital } \\ & \end{array}$

\begin{tabular}{ll} 
pt & pterygoid \\
ptfl & perygoid flange \\
q & qudrate \\
qj & qudratojugal \\
ras & rugose apical surface \\
rma & right mandible \\
ro & root \\
rpr & retroarticular process \\
rt & replacement tooth \\
sa & surangular \\
sc & scratch \\
sof & suborbital fenestra \\
spl & splenial \\
sq & squamosal \\
stf & supratemporal fossa \\
sts & supratemporal shelf \\
sy & symphysis \\
t & tooth \\
tu & tubercle \\
tuc & tuberculated carina \\
uca & upper caniniform tooth \\
ucr & upper crown \\
ve & ventral \\
vsc & vertical scratches \\
wf & wear facet \\
wfc & wear facet on the carina \\
V & trigeminal foramen \\
& \\
\hline &
\end{tabular}

\section{MATERIAL AND METHODS}

\section{Material and illustration}

The following taxa are used in this study: Protosuchia: Edentosuchus tienshanensis Young, 1973; 'Kayenta form’ protosuchian (Clark 1986, Clark and Fastovski 1986, Sues et al. 1994).

Notosuchia: Candidodon itapecuruense Carvalho and Campos, 1988; Malawisuchus mwakasyungutiensis Gomani, 1997; Chimaerasuchus paradoxus Wu, Sues, and Sun, 1995; Simosuchus clarki Buckley, Brochu, Krause, and Pol, 2000; Libycosuchus brevirostris Stromer, 1914; Notosuchus terrestris Woodward, 1896; Sphagesaurus huenei Price, 1950; Sphagesaurus montealtensis Andrade and Bertini, 2008a; Caipirasuchus paulistanus Iori and 
Carvalho, 2011; Mariliasuchus amarali Carvalho and Bertini, 1999; Adamantinasuchus navae Nobre and Carvalho, 2006; Armadillosuchus arrudai Marinho and Carvalho, 2009; Yacarerani boliviensis Novas et al. 2009; Pakasuchus kapilimai O’Connor et al. 2010. Neosuchia: Bernissartia fagesii Dollo, 1883, Unasuchus reginae Brinkmann, 1992; Iharkutosuchus makadii Ösi, Clark, and Weishampel, 2007; Brachychampsa montana Gilmore, 1911; Allognathosuchus haupti Weitzel, 1935; Acynodon iberoccitanus Buscalioni, Ortega, and Vasse, 1997; Acynodon adriaticus Delfino, Martin and Buffetaut 2008; and Caiman latirostris Daudin, 1802.

Morrinhosuchus luziae Iori and Carvalho, 2009 and Labidiosuchus amicum Kellner, Figueiredo, Azevedo and Campos 2011 probably belong to the group of heterodont notosuchians but their remains including their dentition are too poorly preserved to make detailed observations, thus they are not included in this work. Inventory numbers used in the text refer to those specimens that were personally studied, and in most cases molds have been taken from the teeth.

Besides published references, specimens of the Osteological and Comparative Anatomical Collection of the Natural History Museum of Paris were used to compare the dentition of crocodyliforms and extant lizards.

All taxa and most of the specimens used in this study have already been illustrated in the original description of the taxa. In several cases, however, the illustrations are not informative enough, and numerous important details, especially those of the cranial and dental morphology are neither figured nor described. Thus, besides providing new, high resolution images and drawings of the specimens, the current study illustrates those morphological features that are of great importance regarding the functional aspects of these specimens.

\section{Definition of heterodont dention used in this study}


Generally, the dentition of most crocodyliforms can be regarded as heterodont because the shape, size and function of the teeth in each quadrant of the jaws are different; consequently true isodont dentition is rare in this archosaurian group. For example, most crocodyliforms possess in each jaw quadrant at least one hypertrophied caniniform tooth that is a perfect tool for prey capture.

Other, usually conical (mostly carinated) posterior teeth rather have the function of holding the prey in the mouth. A typical trend in the dentition of the group (and actually in most tetrapods) is that the posterior tooth crowns are usually lower, more stocky and massive to crush food item before swallowing it. The reason for that is, the more posterior position the teeth have, the greater bite force can be applied by them, because they are closer to the cranial adductor muscles and the jaw joint (rotation point). Advanced oral food processing is, however, not a characteristic feature of these forms.

Crocodyliforms with ziphodont dentition have more complex teeth with serrated mesiodistal carinae mesiodistally. In these usually terrestrial forms (see e.g. Willis and Mackness 1996) serrated carinae are suggested to help cutting smaller chunks off of the prey during feeding. However, due to the lack of precise occlusion and shearing of the upper and lower teeth, longlasting and efficient intraoral food processing was most probably not present in ziphodont forms.

This study deals mostly with those crocodyliforms which possess marked heterodonty in each quadrant of the jaws. Posterior teeth are usually multicusped or bear other kind of crown ornamentation (e.g. ridges, denticles, tubercles, apical grooves) and strongly differ from the simple conical tooth morphology. This kind of complex tooth morphology is indicative of some higher degree of oral food processing (e.g. shearing, crushing, chewing, grinding the food particles) that is achieved by precise dental occlusion in some forms and by special (not simply orthal) movement of the mandibles in various others. 


\section{Jaw adductor reconstruction}

For the reconstruction and rough comparison of the mass and architecture of cranial adductors among the different taxa, it is assumed that in fossil crocodyliforms the organization of the cranial adductor musculature was basically similar to those of extant ones, and the origin and insertion areas of these adductors were mainly on the same bony surfaces (Busbey 1989). Nevertheless, it has to be noted that the origin or insertion surfaces of some muscles migrated from one bone or region to the other during the evolution of the group. For example, Holliday and Witmer (2009) pointed out that whereas protosuchians and thalattosuchians possess osteological correlates indicative of several muscles (e.g. M. pseudotemporalis superficialis and M. adductor mandibulae externus profundus) that filled the supratemporal fossa („dorsotemporal fossa” of Holliday and Witmer 2009), in more advanced mesoeucrocodylians only M. adductor mandibulae externus profundus occupied the supratemporal fossa. They concluded that „Mm. pseudotemporalis profundus and superficialis must have uncoupled from their plesiomorphic bony attachments, the epipterygoid and dorsotemporal fossa, respectively, and shifted ventrally onto the lateral bridge and postorbital process of the laterosphenoid during mesoeucrocodylian evolution (Holliday and Witmer 2009:731). In addition, dental features, wear pattern and cranial anatomy along with the reconstructed direction of power stroke also suggest some different organization of cranial adductor muscles in some cases, compared to extant forms.

Reconstruction of the cranial adductors (mainly parts of the pterygoid and external adductor muscles, and M. adductor mandibulae posterior) in the fossil crocodyliforms was mainly accomplished by comparison of the position as well as the size and shape of muscle scars on the particular bones with those mentioned in extant crocodilians (Iordansky 1964; Schumacher 1973; Busbey 1982, 1989; Drongelen and Dullemeijer 1982; Cleuren and De 
Vree 2000; Endo et al. 2002; Holliday and Witmer 2007, Figure 4). Furthermore, earlier reconstructions for other extinct forms were also used for comparison (e.g., Mueller-Töwe 2006, Holliday and Witmer 2009, Ösi and Weishampel 2009). However, in contrast with e.g. various mammals (Gambaryan and Kielan-Jaworowska 1995), most muscle scars of the cranial adductors in crocodyliforms are not charaterized by well-developed crests that would clearly indicate the extent and sometimes direction of the attaching muscles or aponeuroses. In crocodyliforms three cranial adductor muscles (M. pseudotemporalis profundus, $\mathrm{M}$. pseudotemporalis superficialis, M. intramandibularis) either originate on very small bony areas or connects to the bundles of other muscles, and, at least in extant forms, they are too weak (see Busbey 1989) and do not play an important role in jaw closure.

In the case of M. pseudotemporalis profundus (MPP) a small slip originates from the lateral bridge of the laterosphenoid, ventral to M. pseudotemporalis superficialis, with some fibers attaching to the ventrolateral surface of the maxillary nerve. This muscle melds with the dorsal fibers of M. pterygoideus dorsalis near the caudodorsal surface of the angular (Holliday and Witmer 2007) so its attachment area cannot be directly studied.

Musculus pseudotemporalis superficialis (MPSS) originates from the posterior surface of the postorbital process of the laterosphenoid, rostral to M. adductor mandibulae externus profundus. It attaches ventrally to the dorsomedial surface of the cartilago transiliens, with some fibers merging with M. adductor mandibulae externus profundus. Finally, M. intramandibularis connects to the ventral portion of M. pseudotemporalis superficialis (Holliday and Witmer 2007, 2009), thus no bony origin surface can be determined in this muscle. In most cases, the epipterygoid-laterosphenoid region being the origin surface of the M. pseudotemporalis group is not well preserved (or covered with sediment), thus their mass and role in the studied fossil forms are more complicated to interpret compared to the pterygoid (MPT) or external adductor muscles (MAME group). Nevertheless, in those forms 
where the bony origin surfaces of M. pseudotemporalis superficialis and profundus are preserved, the significance of these muscles will be discussed.

Terminology of muscular reconstruction follows Holliday and Witmer (2007).

\section{Tooth wear analysis}

Analysis of wear patterns on the teeth of living and extinct vertebrates represents a method that is able to demonstrate possible jaw mechanism, the patterns of tooth-tooth or tooth-food contacts, and potentially the dietary preference of the animal (Greaves 1973, Rensberger 1973). On the wear facets, macrowear and microwear patterns were analyzed. Macrowear is defined here as a wear feature longer than $0.5 \mathrm{~mm}$, microwear features are under this size. Wear patterns of the teeth can mostly be characterized by the combination of two basic features, scratches and pits. Following Ungar (1996), pits are defined as having length-width ratio smaller than $4: 1$. In scratches, this ratio is greater than $4: 1$.

Mapping of macrowear features on the surface of the tooth crowns is applied to reconstruct how the lower tooth row moved against the upper one. Macrowear is informative either they preserved on the enamel or on the softer dentine. In addition, the structure of the enameldentine interface (EDI) is of great importance because it provides information on the direction of mandibular movement during occlusion (Weishampel 1984). A light microscope was used to detect gross macrowear patterns. To reveal the finer details of the macrowear (including EDI) and microwear patterns, a Hitachi S-2600N scanning electron microscope (SEM) was used (for technical details see Walker et al. 1978; Teaford 1988; Fiorillo 1998). In most studied specimens, fine resolution molds were taken from the teeth for SEM studies. Molds of in situ tooth crowns of the different taxa were prepared following the procedure described by Grine (1986) for hominid mammals. Specimens were first cleaned with cotton swabs soaked with ethyl alcohol. Impressions were then made using Coltene President Jet 
Regular (polysiloxane vinyl) impression material, and casts were made with EPOTEK 301 epoxy resin. This procedure reproduces features with a resolution of a fraction of a micron (Teaford and Oyen 1989; El-Zaatari et al. 2005). After light microscopy examination, specimens deemed suitable for further study were sputter-coated with approximately $5 \mathrm{~nm}$ of gold, and examined using SEM at 20-25 kV. Images from the macrowear and microwear sites were taken at magnifications of up to 500x for most specimens.

\section{Jaw mechanism and dental occlusion}

Study of the skull (especially the temporal cavity) and mandible architecture, jaw joint, dental morphology, tooth wear pattern, and the origin and insertion surfaces of the jaw adductors revealed four basic types of jaw mechanisms (powerstroke) among crocodyliforms. Most of these types of mechanisms have already been described or reconstructed in some other groups of vertebrates too (mostly in reptiles and mammals, for a summary of extant correlates see Figure 5), among which the extant forms provide perfect analogues in the reconstruction of jaw mechanism in crocodyliforms. The four main types of jaw mechnisms (Figure 6) and their extant analogues are as follows:

1, Orthal jaw movement. It is characteristic for most crocodyliforms including all extant forms. In this mechanism, the quadrate condyles precisely fit into the glenoid cavity of the articular, and the pterygoid flanges are wide and prevent the lateromedial sliding of the mandibles. Jaw closure works simply in the vertical plane, there is no anteroposterior or lateromedial shifting of the mandibles. The main adductors are the pterygoid muscles. Except for some forms (e.g. Araripesuchus wegeneri [Sereno and Larsson 2009]), dental occlusion does not exist and dental wear is exclusively due to tooth-food-tooth contact. Jaw closure is bilateral which means that the left and right mandibles moved synchronously in every cycle of jaw closure. 
Details of the feeding apparatus and jaw closure of extant forms has been studied in detail by many workers (e.g. van Drongelen and Dullemeijer 1982, Cleuren and De Vree 2000, Endo et al. 2002).

2, Proal jaw movement. One of the jaw closure mechanisms with an anteroposterior shifting of the mandibles. The glenoid surface of the articular is anteroposteriorly elongated and lies well below the level of the occlusal plane. The main adductors are the pterygoid muscles, especially MPTV. Besides the usual orthal mandibular movement, jaw closure is characterized by a protractive powerstroke in which the mandibles are pulled forward to bring the upper and lower teeth into a shearing contact. Occlusion is most probably bilateral. Among extant vertebrates, this type of jaw movement occurs in Sphenodon (e.g. Robinson 1976, Gorniak et al. 1982, Curtis et al. 2011, Jones et al. 2011, 2012) and rodents (e.g. Weijs 1975, Gorniak 1977). In Sphenodon the glenoid surface is anteroposteriorly elongated, the mandibular symphysis is flexible to help the glenoid surface to follow the distal articulation of the quadrate. During occlusion an interdigitating tooth-tooth contact is present when the lower tooth row is positioned between the two upper (maxillary and palatinal) tooth rows. In this phase the mandibles are pulled forward by the pterygoid muscles. This movement produces a shearing mechanism by the cutting edges of the particular teeth that is accompanied by a long axis rotation of the lower jaws (Gorniak et al. 1982, Jones et al. 2012, Figure 5(A-F)).

In rodents, the glenoid fossa (on the skull) is elongate and lateromedially slightly wide (among others this feature allows a lateromedial translation/rotation of the mandibles, see e.g. Gorniak 1977) and the teeth has horizontal or subhorizontal occluding surface. The anterior shifting of the mandibles is motorized by the medial and lateral masseters that have a great anteroposteior component in their orientation (Weijs 1975, Figure 5(G-I)). 
3, Palinal jaw movement. The other type of jaw closure with anteroposterior shifting of the mandibles. The glenoid surface is anteroposteriorly elongated and below or in the level of the occlusal plane. The main adductors are the external adductors (MAME) with their profundus part (MAMEP) especially developed. Jaw closure is charaterized by a retractive powerstroke in which the mandibles are pulled backward by the external adductors to bring the upper and lower teeth into a shearing contact. Similarly to the proal movement, the basic orthal component is also present here, so during occlusion the mandibles are pulled obliquely, i.e. simultaneously upwards and backwards. A semicircular mode of chewing interpreted from curved scratches on the wear facets reported in neoceratopsian dinosaurs (Varriale 2012) is not present in any known crocodyliform. Occlusion can be bilateral or unilateral. In the latter case, jaw closure is complemented with a lateromedial rotation of the mandibles.

Today, retractive powerstroke during jaw closure can be found in gopher tortoises (Bramble 1974). In these forms the glenoid surface is anteroposteriorly elongated and the mandibular symphysis is fused. The main adductors pulling the mandibles backward are parts of the external adductors that have an anteroventral-posterodorsal orientation. During occlusion the mandibles are shifted only backwards. The edentulous jaws are covered with rhamphoteca that is, in some cases, well serrated.

4, Lateromedial jaw movement. The glenoid surface is slightly elongated anteroposteriorly and lies well above the occlusal plane. The lateral pterygoid flanges are reduced. The main adductors are the pterygoid muscles and MAMP among which MPTV is responsible for the lateromedial rotation of the mandibles. Jaw closure starts with an orthal component and the powerstroke is complemented with a lateromedial rotation of the mandibles. In Iharkutosuchus occlusion is most probably unilateral anteriorly and bilateral posteriorly that is due to the size difference of the anterior and posterior teeth. 


\section{RESULTS}

\section{Heterodont protosuchians}

Systematic paleontology

Crocodyliformes Hay, 1930 (sensu Benton and Clark 1988)

Protosuchia Brown, 1934

Protosuchia indet. ('Kayenta form' Clark 1986)

(Figure 7-10)

Referred specimens-UCMP 97638 (?Edentosuchus wellesi of Clark 1986, but see Clark and Fastovsky 1986, Sues et al. 1994), skull with articulated right mandible, UCMP 125395 sectioned cranium with most of the outer surface eroded, UCMP 125871 skull with mandibles, UCMP 125359 eroded, compressed braincase, UCMP 125872 right jugal and maxilla, UCMP 125870 well-preserved braincase.

Locality_Pumpkin Patch locality, near Tuba City, Arizona, USA (Sues et al. 1994). Horizon—Early Jurassic (Late Sinemurian-Pliensbachian?, Sues et al. 1994), Glen Canyon Group, Kayenta Formation.

\section{Cranial and mandibular morphology}

Among the few non-mesoeucrocodylian crocodyliforms that are well represented by cranial material, the Early Jurassic Kayenta form (Clark 1986) and the Early Cretaceous Edentosuchus tienshanensis Young, 1973 possess heterodont dentition with multicusped teeth. As other protosuchians (e.g. Orthosuchus [Nash 1968, 1975], Protosuchus [Colbert and Mook 1951, Gow 2000], Gobiosuchus [Osmolska 1972]), the Kayenta form is small bodied 
with short skull and large, laterally-laterodorsally facing orbits. The rostrum is short, high and, most importantly, narrow mediolaterally (Figure 7). The consequence of the latter feature is that the tooth rows are situated relatively close to each other (Figure 7(D)), thus the oral cavity was quite narrow; a feature that is frequently seen in more derived notosuchians (e.g. sphagesaurids). One of the main differences between the protosuchian and mesoeucrocodylians rostrum is that the protosuchian secondary bony palate is usually not well-developed occupying only a short anterior segment of the snout, but the Kayenta form has a more pronounced secondary palate than other protosuchians. Ventrally the palatines and maxillae are not fused at mid-line, and the secondary choana and the posterior part of the narial passage is wedged between these bones (Clark 1986). In contrast to Orthosuchus (Clark 1986) and Protosuchus (Gow 2000), the palatine of the Kayenta form has a ventromedially extended, massive shelf that is thick laterally and sculptured medially by small pits similar to Shantungosuchus (Wu et al. 1994). These pits could have served as openings for nerves and blood vessels supplying the soft secondary palate (J. Clark 2010, pers. comm.). In the Kayenta form, this extended palatine covered by a soft but perhaps massive secondary palate might have played an important role during puncturing and crushing the food item.

The supratemporal fenestrae of the Kayenta form (UCMP 97638) are anteroposteriorly elongated, moderate in size and they well extend posteriorly above the basicranium to provide significant muscle attachments. As in Protosuchus but in contrast to mesoeucrocodylians, the braincase of the Kayenta form is strongly expanded ventrally and laterally due to the hollow, presumably pneumatized basisphenoid (Clark 1986). Furthermore, the posterodorsal end of the pterygoid attaching to the basisphenoid is well developed posteriorly; a feature rather similar to the pterygoid of modern lizards. Thus the posterior part of the pterygoid and the basisphenoid could play an important role (e.g. in the origin of jaw adductors, see below) in the architecture of the medial part of the adductor fossa in contrast to more advanced 
crocodyliforms. The epipterygoid is present in Edentosuchus (UCMP 125358; Clark, 1994, Holliday and Witmer 2009).

The occipital condyle is two times smaller than the foramen magnum (Clark 1986) and it is oriented posteriorly, slightly posteroventrally. Usually, in more advanced heterodont notosuchians, the occipital condyle faces more ventrally than in the Kayenta form.

The mandibles are short with robust, elevated post-dentary part, and the glenoid surface is in a high position, much higher than the occlusal plane (Figure 7(F)). The massive, elongate symphysis is fused up to the fourth post-caniniform tooth in the Kayenta form. External mandibular fenestrae are small.

In the Kayenta form, the quadrate-articular joint is preserved and the quadrate bears several important features: it is short anteroposteriorly not extending beyond the occiput, the medial and lateral condyles are not separated by a distinct groove as in Shantungosuchus (Wu et al. 1994) and in most mesoeucrocodylians. In addition, its "articulating surface is continuous medially with the otoccipital" (Clark 1986:261) and articulates with the very broad glenoid surface of the articular. This unusal, extended articulation between the medial part of the articular and the otoccipital is also present in Protosuchus haughtoni (Gow 2000) but not in Orthosuchus (Nash 1968). In additon, the glenoid surface of UCMP 97638 appears to be slightly longer anteroposteriorly than the anteroposterior length of the quadrate articulation suggesting the ability of the mandibles to move somewhat anteroposteriorly. Nevertheless, the region of the jaw joint even in the type specimen is too poorly preserved, thus it is not clear if the surangular forms a wall lateral to the glenoid surface to prevent lateromedial translation of the mandibles in contrast to mesoeucrocodylians, or whether this wall is simply not present. 
The Kayenta form possesses a complex, highly heterodont dentition including bicusped postcaniniform teeth (Clark 1986, Figure 8). The anterior teeth are poorly preserved. The four premaxillary teeth are conical, straight with relatively rounded tip and do not bear any serrations or keels. Their enamel surfaces bear longitudinal wrinkles (Clark 1986). The four anterior dentary teeth of the Kayenta form are poorly preserved but they are blunt and conical in shape, and slightly increase in size posteriorly. The fourth is the largest that fits into the much wider notch between the premaxilla and maxilla. The fourth dentary tooth does not appear to be as robust and large as the typical lower caniniforms seen in Edentosuchus or in Protosuchus, however, this might simply be a preservational artifact (J. Clark, 2012 pers. com.).

The post-caniniform teeth (Figure $8(\mathrm{~A}-\mathrm{C})$ ) are not so closely packed as in Edentosuchus. In the Kayenta form the first maxillary tooth, behind the anteroposteriorly enlarged notch between the maxilla and premaxilla, is small and conical (well observable in UCMP 97638). It is worn but the crown surface on its distal side is constricted indicating that this tooth was also bicusped with a smaller medial cusp. Posterior to this, the left and right tooth rows slightly diverge, and the teeth in this section are transversely widened, bicusped and similar in size except for the last one that is smaller than the rest. Both the upper and lower teeth are constricted at their base, and the labiolingual axis of the tooth crowns is slightly offset relatively to the transverse plane but is perpendicular to the lateral edge of the dentary and maxilla following their posterolateral curvature (J. Clark 2012 pers. com.).The orientation of the labiolingual axis of the tooth in the Kayenta form differs from that found in the extremely elongated symphysis of several notosuchian forms (e.g. Adamantinasuchus, Sphagesaurus, Armadillosuchus, see below) where this tooth-axis is oblique relative to the lateral surface of the dentary and maxilla. The occlusal surface of the upper teeth in the Kayenta form is directed slightly lingually, whereas that of the lower teeth has a 
complementary, labial inclination. A large gap (in some cases half of the width of a crown) is present between the bicusped teeth. In the symphyseal part of the lower jaw, the tooth rows are situated very close to each other, thus producing a relatively small space for the oral cavity and the tongue (Figure 7(E)).

During the evolutionary history of reptiles, transversely oriented, bicusped teeth occured numerous times, for example, in some therocephalian synapsids (Kemp 2005) and also in some procolophonians (King 1996, Fraser et al. 2005), although in these groups labial and lingual cusps are not always in the same plane and in some cases smaller cusps surround one of the main cusps. Dental morphology similar to that of the Kayenta form cannot be found among extant crocodylians. In lepidosaurs there is an extreme diversity in dental morphology, and dentition approximately similar to that of the Kayenta form (or any other crocodyliforms) can be found more easily. Polyglyphanodontines (Polyglyphanodon sternbergi Gilmore, 1940, Peneteius aquilonius Estes, 1969, and Bicuspidon numerosus Nydam and Cifelli, 2002, 2005; Nydam 1999, Nydam et al. 2000) from the Cretaceous of North America have transversely oriented, lingually expanded, and mesiodistally compressed teeth. They have been described as herbivorous or omnivorous forms. In these forms, however, the labial and lingual cusps are not as rounded as in the Kayenta form, and their teeth are more chisel-like with a developed, sharp crest between the two cusps. Among extant forms, the herbivorous Dicrodon guttalatum (Kosma 2004, Nydam 1999) possesses oval, transversely oriented, bicusped/multicusped teeth with two pyramid-shaped main cusps but these teeth are not as wide transversely as those of polyglyphanodontines. Other teiid lizards such as Teius teyou or Ameiva bifrontata have transversely oriented, bicusped teeth posteriorly (Kosma 2004, Brizuela and Albino 2009). Teius teyou feeds on insects and spiders but it is partly also frugivorous (Brizuela and Albino 2009). 
The posterior bicusped teeth of the Kayenta form are heavily worn (Sues et al. 1994, Figure 7, 8), which can be explained by occlusion of the upper and lower teeth during food processing. Unfortunately, the anterior dentary and premaxillary teeth are too poorly preserved to provide useful information on the wear in this region. All teeth in the maxilla and teeth posterior to the largest dentary tooth bear extensive wear facets and show different stages of wear. It is important to note that the enamel coat on the teeth is relatively thin thus the dentine is frequently exposed. Wear on the first, monocusped or perhaps bicusped conical maxillary tooth is due to horizontal erosion of the tip. Posterior to this tooth, wear facets are extended on both cusps, transversely wider than mesiodistally long, slightly concave in the middle of the crown and facing slightly lingually (Figure $8(\mathrm{E}, \mathrm{F})$ ). The wear facets on the bicusped dentary teeth are complementary to the maxillary teeth and face slightly labially-mesiolabially, and otherwise have similar wear facets. In the earlier stage of wear $\left(3^{\text {rd }}\right.$ right maxillary tooth, $5^{\text {th }}$, $6^{\text {th }}$ left dentary teeth of the UCMP 97638) the wear facet is V-shaped and wear facets appear only on the cusps; the ridge between them is only slightly eroded (Figure $8(\mathrm{D})$ ). On the labial cusp of the third, right maxillary tooth of UCMP 97638 the dentine of the cusps is exposed and it forms a slightly convex core that is more eroded marginally close to the enamel (Figure 8(D)). The enamel-dentine interface (EDI) is not flush but rather rugged, and a step morphology is present. The dentine surface bears some heavy pits. On the lingual cusp, the dentine is still not exposed only the enamel cover of the cusp is slightly eroded forming a labially facing surface. This enamel surface is irregular with deep pits rather than scratches. Wear at this stage is similar to that seen in some extant teiid lizards with bicusped posterior teeth (e.g. Dicrodon, Teius). Here, wear facets are found on tips and cusps of all tooth types and the cusps are worn down in a rugged manner (Brizuela and Albino 2009). 
In a more advanced stage of wear, cusps are completely eroded and the V-shaped facet becomes more uniform, extended and slightly concave facing very slightly lingually on the upper teeth as seen on the $4^{\text {th }}$ and $5^{\text {th }}$ right maxillary teeth of UCMP 97638. The EDI is also smoother on all sides of the crowns, but the eroded enamel surface is still slightly irregular. The eroded dentine surface is ornamented by a high number of irregular pits, and rarely some short scratches also occur. Some short, mesiodistally oriented scratches are present on the enamel of the mesial surface of the $4^{\text {th }}$ right maxillary tooth (Figure $8(E-G)$ ).

\section{Adductor muscle reconstruction}

Although there are some important differences between protosuchian and mesoeucrocodylian pterygoid bones, that of protosuchians in general was an anteroposteriorly extended element with medium-sized lateral flange which provided important attachment areas for the pterygoid muscles. This indicates that the typical crocodyliform adductor muscle construction, in which the pterygoid muscles are the dominant adductors (Figure 4), was already present in protosuchians (Iordansky 1964). In the Kayenta form, M. pterygoideus dorsalis (MPTD) appears to have been less developed than M. pterygoideus ventralis (MPTV) similarly to modern crocodylians (Busbey 1989). The bony origin surfaces of MPTD such as the maxillapalatine articulation, the dorsomedial surface of the palatine, the ventrolateral surface of the lacrimal, the dorsomedial surface of the maxilla-ectopterygoid articulation, the margin of the suborbital fenestra, the prefrontal pillar, the dorsolateral surface of the palatine and the ascending process of the pterygoid in modern forms (Busbey 1989, Holliday and Witmer 2007) appear to be relatively smaller in the Kayenta form than those seen in modern forms (e.g. Alligator mississippiensis). One reason for this is that the posterior part of the rostrum, especially at the level of the very small suborbital fenestra, is narrower than in platyrostral crocodyliforms (Ösi et al. 2010:fig. 4). The insertion area of MPTD (i.e. the ventromedial part 
of the angular and articular, ventral to the glenoid fossa in modern forms [Holliday and Witmer 2007]) is poorly preserved but does not seem to be particularly well developed below the glenoid surface and external mandibular fenestra.

MPTV could have been the dominant adductor as it is in extant crocodylians where it arises from the posterior rim and the caudolateral surface of the lateral pterygoid process (Holliday and Witmer 2007; Figure 4). The lateral pterygoid process in the Kayenta form is relatively wider lateromedially than that of lizards (compared, for example, with Polyglyphanodon sternbergi [Gilmore 1940]) but not as extended laterally and posteriorly as in modern crocodyliforms. Nevertheless, posteriorly the pterygoids are well extended, more similarly to lizards, forming a significant part of the expanded basicranium. This surface might have served as additional surface of origin for MPTV, in contrast to modern crocodylians (Figure 9). In modern forms, MPTV wraps around the retroarticular process to insert onto the caudolateral surface of the angular (Holliday and Witmer 2007). Clark (1986) noted that in the Kayenta form this part of the angular is flat and probably the attachment area of MPTV did not extend to its lateral surface. This region is not as extended and massive as in extant forms indicating the relatively smaller mass of MPTV compared to modern crocodylians. This is also in accordance with the small size of the lateral pterygoid processes.

M. adductor mandibulae posterior (MAMP) is an important, roughly vertically oriented adductor muscle in extant forms that arises from the central, posterior and medial parts of the ventral surface of the quadrate (Busbey 1989; Holliday and Witmer 2007). Its fibers attach to the anterior surface of the articular, the dorsal surface of the angular (Busbey 1989) and the medial/internal surface of the fascia covering the external mandibular fenestra (Holliday and Witmer 2007; Figure 4). In the Kayenta form, no crest like that seen in modern crocodylians (Iordansky 1973) occurs on the ventral surface of the quadrate but laterally the quadrate is bordered by a steep ridge the lateral surface of which leads to the supratemporal fossa. This 
ridge could have provided additional insertion surfaces for MAMP (Figure 9). These cranial features suggest that similar to extant forms, MAMP was an important but not an extraordinarily advanced adductor muscle in the Kayenta form.

Just anterolateral to the origin surface of MAMP on the anteroventral surface of the quadrate, and from the shallow trough leading dorsally to the supratemporal fossa arise the fibers of M. adductor mandibulae externus medialis (MAMEM) and they attach on the medial part of the surangular, just ventral to the insertion of MAMES (Busbey 1989; Holliday and Witmer 2007). In the Kayenta form, the origin surface of MAMEM is an almost vertically oriented, slightly convex surface leading into the supratemporal fenestra. The post-dentary part of the UCMP 97638 mandible is poorly preserved but based on the interpretation of Clark (1986: fig. 14) its dorsal margin is composed mainly of the surangular, that is anteroposteriorly elongated, which would provide extended attachment surface for MAMEM. In modern crocodylians the fibers of $\mathrm{M}$. adductor mandibulae externus superficialis (MAMES) arise from the ventrolateral surface of the quadrate and quadratojugal (Holliday and Witmer 2007; Figure 4) lateral to the attachment surfaces of MAMP and MAMEM, and also from the postorbital close to the postorbital bar (Busbey 1989). MAMES attaches to the dorsal margin of the surangular (laterodorsally to MAMEM). In the Kayenta form, the quadratojugal is lateromedially wide and posteriorly pronounced, indicating relatively large attachment areas for MAMES.

In modern crocodylians the fibers of the profundus part of M. adductor mandibulae externus (MAMEP) take their origin from the margin of the supratemporal fenestra (lateral, shelf-like surface of the parietal and anterior surface of the squamosal, Holliday and Witmer 2007; Figure 4). In the Kayenta form, the posterior and posterolateral surfaces of the supratemporal fenestra are not as extended as in Edentosuchus indicating moderate-sized insertion surfaces for MAMEP (Figure 9). In modern forms, cartilago transiliens (Busbey 1989) and the 
rostrodorsal edge of the surangular provide insertion for the tendinous MAMEP (Holliday and Witmer 2007). This part of the mandible is in a highly elevated position in the Kayenta form, and certainly had served as tendinous attachment areas, however, due to preservation no more details can be inferred.

Holliday and Witmer (2009) reported an anteroposteriorly extended laterosphenoid in UCMP 125358 with a large pseudotemporalis fossa on its posterodorsal surface. These features let them to conclude that, similarly to other protosuchians and some early mesoeucrocodylians, the Kayenta form also had a M. pseudotemporalis superficialis (MPSS) that occupied the rostral portion of the supratemporal fossa. This also indicates that this muscle, although not as significant as parts of MPT and MAME, could have been comparable in mass to that of extant forms.

To sum up, the origin and insertion surfaces of the jaw adductors in the Kayenta form indicate a mandibular adductor system basically similar to that of extant crocodylians. The roughly horizontally oriented pterygoid muscles could have been the most important adductors with the dominance of MPTV, although they were most probably not as developed as in modern forms. MAMP, MAMES, MAMEM and perhaps MPSScould have been proportionally similar to those of extant forms and MAMEP appears to have been a reduced muscle in the Kayenta form.

\section{Jaw mechanism and possible phases of chewing}

The transversely oriented, bicusped and heavily worn post-caniniform teeth of the Kayenta form indicate effective oral processing of food. Because of this, the Kayenta form represents the earliest crocodyliform with oral food processing and indicates that complex dentition and specialized feeding strategies appeared already during the very early divesification of the group; a major evolutionary pattern that similarly existed in early pterosaurs (Ösi 2011) and 
dinosaurs (e.g. Barrett 2000, Galton and Upchurch 2004). Reconstruction of the relative position of the lower and upper dentition in the Kayenta form clearly shows that they were in an overlapping and thus in occluding position. This is supported by the extensive, slightly concave wear facets of the posterior teeth. The occlusion surface is not completely horizontal but approximately $14^{\circ}$ relative to the horizontal plane (Figure 10). The lower tooth row was positioned slightly lingually compared to the upper one. The transversely oriented tooth crowns with one-one cusp labially and lingually connected by a short ridge are similar to the teeth of polyglyphanodontines but in the latter group a prominent, sometimes serrated blade connects the two cusps (e.g. Polyglyphanodon). In Dicothodon, another polyglyphanodontine, where tooth crowns are not as transversely wide as in P. sternbergi, teeth possess apical wear facets (Nydam 1999), but they are not as extensive as in the Kayenta form. In P. sternbergi, the transversely oriented upper and lower teeth came into an interdigitating configuration during jaw closure (Nydam and Cifelli 2005:fig. 4). In the extant teiid Teius, having transversely oriented, bicusped teeth, a similar interdigitation was described because wear is restricted to the distal ends of the cusps and no wear occurs on the shelves. Whether this configuration was also present in the Kayenta form at least in one phase of jaw closure, or the cuspidate tooth crowns occluded with each other during biting, is not clear, because the degree of anteroposterior movement of the mandibles is difficult to estimate. The lack of elongated and well-oriented scratches on the horizontal wear facets of the posterior teeth indicate that during occlusion of the upper and lower teeth there were no notable anteroposterior or lateromedial components of the lower jaw movement. This is further supported by the lack of wear facets with different orientations (e.g. mesial and distal wear facets) on a single tooth crown. Nevertheless, the wide and anteroposteriorly extended wear facets with approximately uniform orientation (Figure 8,10$)$ suggest a relatively more mobile jaw articulation than that seen e.g. in extant crocodylians. In other words, the single, slightly 
concave and extended wear facet (e.g. the $4^{\text {th }}$ right maxillary tooth of UCMP 97638) shows that jaw closure during food processing was not always as precise as in extant carnivorous forms, and besides food particles the upper tooth certainly occluded with different (e.g. distal, mesial) parts of the opposite lower tooth crown. In conclusion, although there was no preferred additional component of mandibular movement (e.g. proal, palinal, developed lateromedial translation/rotation) that completed the simple orthal jaw closure, there could be some sort of anteroposterior or lateromedial sliding that resulted in a more efficient occlusion and food processing in the Kayenta form.

Edentosuchus Young, 1973

Edentosuchus tienshanensis Young, 1973

(Figure 11-12)

Holotype-IVPP V3236 $(1,2)$, fragmentary skull roof, fused anterior part of the mandibles, isolated left dentary with teeth, fragmentary quadrates+quadratojugal (originally referred as articular+angular), cervical vertebrae (Li 1985).

Referred specimens - GMPKU-P 200101, partial skull with articulated fragmentary mandibles (Pol et al. 2004).

Type Locality_-10-20 km southeast of Urho, Xinjiang Province, Central Asia, Northwest China (Pol et al. 2004).

Type Horizon-Early Cretaceous Tugulu Group.

Cranial and mandibular morphology 
In Edentosuchus the skull is short and the relatively large orbits are facing laterally, laterodorsally. The rostrum is short, high and mediolaterally narrow similarly to the Kayenta form and other protosuchians. Most of the palatal region is hidden by the articulated mandibles in GMPKU-P 200101, thus the medial extension of the maxillae and palatines is unknown. Mandibles are short and massive with elongate symphysis that is fused up to the penultimate tooth (Figure 11,12). The post-dentary part is strongly elevated and the external mandibular fenestra is much larger than in the Kayenta form. Similarly to the Kayenta form, the glenoid surface is in an elevated position, well above the occlusal plane.

The supratemporal fenestrae of Edentosuchus (IVPP V3236) are anteroposteriorly elongated and well extend posteriorly above the basicranium to enlarge muscle attachment areas. The quadrate-articular joint is poorly known. Li (1985:fig. 1D) figured a bone and identified it as a fragmentary angular and articular with the retroarticular process. In the original type material (IVPP V3236), however, two fragments are present, both representing the same region of the left and right sides. In my opinion these bones are not from the posterior part of the mandible but from the left and right fragmentary quadrate (including the quadrate condyles) and quadratojugal. Posteriorly, the quadrates appear to be slightly longer than those in the Kayenta form and curve posteroventrally. The distal quadrate articulation is similar to that of the Kayenta form in the absence of any groove between the medial and lateral condyles. In contrast to a typical crocodylian articulation, it is convex and the articular surface is wider dorsoventrally than that of the Kayenta form. In addition, the articular surface did not continue on the otoccipital as in the Kayenta form.

\section{Dentition and possible extant analogues}

Edentosuchus (Young 1973, Li 1985, Pol et al. 2004) also possesses complex, highly heterodont dentition with multicusped post-caniniform teeth (Clark 1986, Sues et al. 1994, 
Figure 11, 12) but the tooth morphology is strongly different from that of the Kayenta form. The anterior teeth are poorly preserved; only one crushed, upper anterior tooth is known from the last position of the premaxilla that was a conical, probably non-serrated tooth. In the dentary, the small anteriormost tooth is oriented anterolaterally and has three weekly developed, anteroposteriorly aligned cusps with the central cusp being the largest. This tooth is followed by an enlarged conical caniniform that is devoid of serrations and cusps, but bears longitudinal wrinkles on the enamel (Pol et al. 2004, Figure 12).

The post-caniniform teeth of Edentosuchus are more closely packed than in the Kayenta form. Teeth become slightly larger posteriorly and the posterior three maxillary and five dentary teeth are more rounded (Figure 11, 12) compared to the rougly similar-sized post-caniniform teeth of the Kayenta form. The first two anterior of the post-caniniform maxillary teeth of Edentosuchus are tricusped with the central cusp being the largest (cusp angle approximately $60^{\circ}$ ) that is connected with crests to the secondary cusps. These teeth are slightly compressed labiolingually. The opposite teeth in the dentary are also cuspidate. Here, the first postcaniniform is tricusped with anteroposteriorly aligned cusps. The second post-caniniform dentary tooth bears two cusps mesio- and distolabially (Pol et al. 2004). Behind them, teeth become more rounded, stocky and bear more cusps in contrast to the posterior teeth of the Kayenta form.

The three posteriormost maxillary teeth of Edentosuchus are nicely preserved and their crowns are almost in connection both on the holotype and on GMPKU-P 200101 (Pol et al. 2004; Figure 12(E, F)). They bear a large central cusp that is surrounded by smaller marginal cusps in slightly different ways. It is evident on the holotype that the last three teeth in the maxilla are so closely packed that they form an almost continous cuspidate surface for processing the food items. The radical difference of the crown morphology between the 
second (labiolingually compressed, tricusped) and third (stoky, multicusped) maxillary teeth is quite unusal even among heterodont reptiles.

The labiolingually slightly compressed tricusped anterior maxillary and dentary teeth of Edentosuchus are similar to the posterior cuspidate teeth of various iguanid lizards (e.g. the primarily herbivorous Ctenosaura acanthura [MNHN 1909 524]; the omnivorous Basiliscus basiliscus [MNHM 1888 124] or the insectivorous Polychrus sp. [MNHM 1888 600]). Concerning the posterior multicusped teeth of Edentosuchus, no extant lizards possess similar, stocky teeth with multicusped crowns. The primarily herbivorous Tupinambis rufescens (MACN uncatalogued), the molluscivorous Dracaena guianensis (Kosma 2004) or Hemisphaeriodon gerrardi (Kosma 2004), as well as the omnivorous Tiliqua scincoides (MNHN 1898 285), Tiliqua gigas (Kosma 2004), Tiliqua rugosa ('Trachydosaurus rugosus' MNHN B 1885) possess bulbous, crushing teeth posteriorly. These teeth are stocky and massive in a similar manner to those of Edentosuchus, but they are not ornamented by cusps occlusally, thus they rather resemble the rounded, bulbous teeth of globidont neosuchians. The dentition of the teid lizard Ameiva plei shows some similarities with the post-caniniform dentition of the Edentosuchus. In the adult specimens of this extant lacertid, the posterior multicusped dentition is also composed anteriorly of tricusped teeth and posteriorly of globular teeth (Kosma 2004).

\section{Dental wear}

Besides dentition, the two heterodont protosuchians strongly differ in their dental wear features that can well be explained by their different dental occlusion pattern (see below). The type of Edentosuchus contains only mandibular teeth. Most teeth have much lower tooth crown than the others (e.g. left post-caniniform teeth relative to the last tooth, Figure 11(D)) that might refer to significant food processing. It is ambiguous, however, whether their 
occlusal surface possesses wear facets or this strongly irregular, occlusal surface is a preparational artifact because the specimen is covered with thick conservation material. The more complete specimen described by Pol and colleagues (2004) preserved both upper and lower teeth that however, do not show an occlusion pattern (Figure 12). Here, although the enamel is thicker than in the Kayenta form (Pol et al. 2004), tooth crowns appear to be complete and devoid of heavy dental wear. Unfortunately, I had no opportunity to take molds of the teeth to investigate microwear features in this specimen.

\section{Adductor muscle reconstruction}

In Edentosuchus, a great part of the origin and insertion surfaces of the mandibular adductors cannot be reconstructed because the posterior part of the skull and mandibles is poorly known. However, as in the Kayenta form and modern crocodylians, MPTD could have been less developed in Edentosuchus than MPTV because, similarly to the Kayenta form, the posterior end of the nasal cavity and the antorbital fossa is narrow lateromedially providing smaller attachement areas for this muscle. Although three mandibular fragments are known in Edentosuchus, the insertion areas of MPTD are not preserved. The lateral pterygoid process as the origin surface of MPTV is wide, extends laterally rather than lateroventrally (Pol et al. 2004) and appears to be more robust than that in the Kayenta form, indicating more advanced MPTV for this Early Cretaceous protosuchian. The insertrion surfaces of MPTV are not preserved.

In Edentosuchus the ventral surface of the quadrates (originally described by Li [1985:fig. 1D] as the posterior end of the mandible) as the origin surfaces of MAMP bear a developed anteroposteriorly oriented bony crest. This crest appears to correspond to the muscle scar B of Iordansky (1973) and served as the attachment area of this muscle. The insertion surfaces of MAMP on the mandible are not preserved in Edentosuchus, but the crest on the ventral 
surface of the quadrates indicates developed bundles of this muscle comparable to that of modern crocodylians, and it can be concluded that it was an important but not an extraordinarily advanced adductor muscle.

Concerning the external adductors, the origin surface of MAMEM in Edentosuchus cannot be studied. Nevertheless, the post-dentary part of the mandible is strongly elevated in all three specimens, and the dorsal and medial parts of the surangular, at least in the larger, type specimen and in the specimen described by Pol et al. (2004) are massive, lateromedially wide, and on the latter specimen this region is anteroposteriorly elongate. This suggests an insertion surface for MAMEM and MAMES relatively similar in size to that of extant forms. The left quadratojugal, which is the origin surface of MAMES, is preserved on the newly described specimen of Edentosuchus (Pol et al. 2004: fig 1) but cannot be studied because the mandible is in an articulated position and the matrix covers the ventral surface of the quadratojugal. The two quadrate-quadratojugal fragments of the Edentosuchus type material (IVPP V3236) indicate a lateromedially wide, posteriorly pronounced quadratojugal, similar to that seen on the left side of the type of the Kayenta form and suggest large attachment areas for MAMES in Edentosuchus. In contrast to the Kayenta form, the posterior and posterolateral surfaces of the supratemporal fenestra are strongly extended in Edentosuchus suggesting that the insertion surfaces for MAMEP was larger than in the Kayenta form (Figure 11 (A)).

Summarizing the scant information on the mandibular adductors of Edentosuchus, it can be concluded that MPTV could have been the dominant adductor and, based on the comparison of the pterygoid wings of the two heterodont protosuchians, MPTV was probably relatively more developed than that in the Kayenta form. The vertically oriented MAMP was also an important muscle, and MAMEP appears to have been more developed in Edentosuchus than in the Kayenta form. 
Jaw mechanism

The skull and mandibles of the recently described specimen of Edentosuchus (Pol et al. 2004) are preserved in a closed articulation with the lower tooth crowns not occluding with the upper ones, but being positioned just lingual to them. This arrangement of the tooth rows along with a strongly fused symphysis suggests a kind of shearing mechanism of the teeth that is conceivable at least in the case of the anterior, labiolingually compressed, tricusped molariform teeth. The question whether this was the normal position of the lower teeth with no regular occlusion, at least not at the level of the posterior, multicusped teeth or if there was any kind of special mandibular movement (complementing the orthal movement in different phases of chewing) cannot be answered because the jaw joint and the details of wear pattern are unknown in Edentosuchus. Nevertheless, the absence of heavy wear on the teeth of GMPKU-P 200101 might indicate the lack of dental occlusion and any non-orthal mandibular movement, as well as the possible erosional surfaces on the teeth of the type material, are due to tooth-food contact.

\section{Heterodont notosuchians with proal jaw movement}

Remarks to the use of the terms propalinal, proal and palinal

To indicate the occurrence of anteroposterior mandibular movement in some crocodyliforms, different authors (e.g. Clark et al. 1989, Wu et al. 1995, Wu and Sues 1996, Gomani 1997, Fiorelli and Calvo 2008) used different terms like proal, palinal, or propalinal. The term propalinal actually refers to the anteroposterior mandibular movement in general, and it does not differentiate the particular phases of jaw mechanism, especially during the powerstroke. Weijs (1975) and Gorniak et al. (1982) for example, applied the term propalinal in extant animals, but they precisely described the details of the different phases of mastication. In fact, the term propalinal covers two different mechanisms: one being based on proal, the other on 
palinal movements (see Figure 5, 6). As demonstrated by Clark et al. (1989), proal movement occurs when the mandibles move forward during jaw closure in the powerstroke. Of course, in some subsequent phase of the cycle the mandibles have to move backwards, but this retraction occurs in a non-occluding, usually jaw opening phase. Palinal movement, which is the opposite of proal movement, was precisely described by Bramble (1974) in gopher tortoises. Although Bramble did not apply the term palinal but instead simply used the terms protraction-retraction, he clearly demonstrated that in the powerstroke, a ,powerful mandibular retraction" (i.e. palinal movement) occurs during oral food processing (Bramble 1974:107). Following this explanation, here I use proal movement for protractive and palinal for retractive powerstroke.

Systematic Palaeontology

Mesoeucrocodylia Whetstone and Whybrow, 1983

Notosuchia Gasparini, 1971

Malawisuchus Gomani, 1997

Malawisuchus mwakasyungutiensis Gomani, 1997

(Figure 13-15)

Holotype-MAL-45, an almost complete, articulated skeleton (Gomani 1997).

Referred specimens-MAL-45 articualted skeleton; MAL-46 and MAL-47 skulls and jaws; MAL-48 skull and partial skeleton; MAL-49 and MAL-155 skulls and skeletons; MAL-99 skull; MAL-50, MAL-51 and MAL-52 left maxillae; MAL-178 skull and femur; MAL-177 articulated postcranial skeleton; MAL-68, MAL-70, MAL-81, MAL-122 and MAL-163 teeth; MAL-40, MAL-56, MAL-66, MAL-74, MAL-80 and MAL-82 tooth fragments; MAL-100, 
MAL-101, MAL-102 and MAL-103 partial skeletons; MAL-96, MAL-107 and MAL-179

vertebrae; MAL-76 vertebra fragment; MAL-97 and MAL-168 femora (Gomani 1997).

Type Locality-Mwakasyunguti area, Malawi, Africa (Gomani 1997).

Type Horizon-Early Cretaceous, Aptian.

\section{General cranial and mandibular morphology}

Similarly to other heterodont crocodyliforms, Malawisuchus was a small-bodied animal with small, relatively short, narrow and high skull (Figure 13). External nares are facing anteriorly with overhanging nasals extending far anteriorly (Gomani 1997) and orbits are laterallydorsolaterally oriented as it is typical for most notosuchian forms (Gasparini 1971). An important difference compared to heterodont protosuchians (and also to the eusuchian Iharkutosuchus) is that the rostrum does not become abruptly narrow at the level of the antorbital fossa but it progressively tapers anteriorly. In addition, the secondary bony palate is closed up to the pterygoids and the secondary choana opens posteriorly between the palatine and pterygoid as a large, anteroposteriorly long opening divided by a septum (Clark et al. 1989, Gomani 1997). The vomer does not participate in the formation of the palate. The pterygoid has a wide lateral wing that is more developed than in heterodont protosuchians. Posteriorly, the pterygoids do not have a posteromedial ascending process as in some extant crocodylians (Gomani 1997) and they do not have an elongate overlapping posterior process (as seen in the Kayenta form) that has an extended contact with the basisphenoid. Ventrally, the basisphenoid has a narrow exposure in contrast to the expanded, most probably pneumatized basisphenoid seen in the Kayenta form.

The supratemporal fossa is relatively narrow and dorsally it opens in a small, anteroposteriorly elongate supratemporal fenestra. The supratemporal fenestra is situated strongly anterolaterally and it is widely marginated by the postorbital (Figure 13A). The 
lateral temporal fenestra is large and triangular bordered anteriorly by a thin, rod-like postorbital-jugal bar. The occipital condyle is oriented ventrally-posteroventrally (Gomani 1997). The quadrate has a posteroventral orientation. This orientation is much closer to the vertical plane than that in extant crocodylians. In contrast to heterodont protosuchians, the medial and lateral condyles of the quadrate in Malawisuchus are separated by a wide groove. The mandible of Malawisuchus is massive with a post-dentary part longer than the toothed part of the dentary and a large external mandibular fenestra is present (Figure 13C, E). In contrast to heterodont protosuchians, the glenoid surface is in a deep position, much lower than the occlusal plane. As it was discussed in detail by Clark et al. (1989), Malawisuchus possesses a specialized quadrate-mandibular joint that differs from those seen in extant crocodylians but similar to other, recently described notosuchians (see below). Relative to its width, the glenoid surface is two times longer than in extant forms and this articulation surface is not bordered posteriorly by a buttress. As it can be observed in specimen MAL-49, the mandibular symphysis is fused.

\section{Dentition and possible extant analogues}

The strongly heterodont dentition of Malawisuchus consists of smaller, conical anterior teeth, one enlarged caniniform (only in the upper jaw) and several multicusped post-caniniform teeth (Figure 13(D-G)). The premaxilla bears four, slightly lingually curved, conical teeth among which the third is the largest (Gomani 1997). This is followed by the anterior maxillary teeth, the first of which is a small conical tooth and the second is an enlarged posteriorly curved caniniform. In contrast to the anterior part of the upper jaw, the mandible does not possess teeth anteriorly and, unlike Edentosuchus, it is also avoid of an enlarged caniniform. The first mandibular tooth is conical with a weak cingulum. Posterior to the conical teeth, in both the upper and lower jaws the teeth are multicusped with a main central 
cusp surrounded by smaller and larger cusps in different arrangements (Figure 13(F, G)). In the maxilla, four multicusped teeth (Gomani 1997) occur with developed cuspidate cingula mostly on the distal side of the crown. These posterior teeth are closely spaced and except for the last one they are inclined posteriorly. In the first multicusped maxillary tooth, the cuspidate cingulum is not so much developed. The second, largest multicusped tooth has numerous accessory cusps, poorly developed mesial cusp and a large, mesiodistally wide distal cusp at the base of the central cusp. The central cusp bears a sharp, straight ridge distally extending from the apex to the base of the crown (Clark et al. 1989). Important feature is that the mesial part of the central cusp of the maxillary teeth never exhibits any ridge but it is a rounded and smooth surface. Gomani (1997) reported seven accessory cusps and the central cusp in the third multicusped maxillary tooth. Secondary cusps both mesially and distally are well developed but no accessory cusps are present on the labial side (Gomani 1997:fig. 3F, Jacobs et al. 1990:fig. 3). A fourth, small multicusped maxillary tooth is present (contra Clark et al. 1989). This tooth has a relatively large mesial accessory cusp and six additional cusps distal to the central cusp.

The number and exact morphology of the mandibular teeth of Malawisuchus is not completely clear because this tooth row is always preserved lingual to the upper one, mostly in the matrix. Behind the suggested first, conical tooth, multicusped teeth are present with mesial, labial and distal accessory cusps. In these teeth, the sharp straight ridge of the central cusp is on the mesial side and the teeth lean slightly mesially (Clark et al. 1989), similarly to the anterior mandibular teeth of sphagesaurids (see below).

Gomani (1997) provided evidence that the dental morphology of Malawisuchus changed during ontogeny. This is supported by the different cusp development among individuals, and in addition by the tooth morphology of a small mandible fragment (associated with unfused vertebrae) which possess four conical teeth (plus two empty alveoli posteriorly). Similar 
ontogenetic changes in the dental morphology have also been reported in several extinct (Estes and Williams 1984) and extant (Kosma 2004) lepidosaurian taxa.

As extant analogues for the dentition, especially for the multicusped teeth of Malawisuchus, teeth of heterodont lepidosaurs, again, are the best examples. Nevertheless, besides the mesial and/or distal accessory cusps, a central cusp with numerous labial and lingual cusps in the multicusped teeth of Malawisuchus is not typical in lepidosaurs. One rare example among extant scincomorphan lizards that possesses bicuspid/multicusped teeth is Dicrodon guttalatum. In this herbivorous species, posterior mandibular teeth have two enlarged cusps (apical angle $70^{\circ}$ ) with smaller cusps labially. Here, however, two, approximately transversely oriented main cusps (as it was discussed in the Kayenta form) are present and not one single central cusp as in Malawisuchus. The different species of the predominantly insectivorous teiid Ameiva have generally similar, labiolingually slightly compressed teeth with a main cusp and, depending on the species and the position of the tooth, with one mesial (e.g. Ameiva corax, Ameiva chrysolaema boekeri, Ameiva exsul desechensis, Kosma 2004) and in some cases one distal cusp (e.g. Ameiva plei, Ameiva griswoldi, Ameiva $p$. pluvianotata, Kosma 2004). However, in Ameiva no additional cusps occur labiolingually. In Malawisuchus, the pointed central cusp of the multicusped teeth has an apical angle usually between $40^{\circ}$ to $50^{\circ}$. This relatively small angle is essential for reduction of contact area between tooth and food to penetrate and crush hard food items (such as the exoskeleton of insects as it was pointed out by Evans \& Sanson 1998, Herrel et al. 2004, Kosma 2004). Thus, based on tooth morphology, a predominantly insectivorous habit can be inferred for this small-bodied terrestrial crocodyliform, as it was also suggested earlier (Jacobs et al. 1990, Jacobs 1993).

\section{Dental wear}


Extensive dental wear has been documented on both the upper and lower multicusped teeth of Malawisuchus (Clark et al. 1989, Gomani 1997). Unfortunately, I could not study the specimens personally, thus, apart from the description provided by the previous authors, no more details are available at present on the wear patterns (e.g. wear pattern on the anterior, caniniform teeth, composition of microwear and macrowear, scratch orientations, enameldentine interface on the multicusped teeth). The multicusped teeth frequently possess apical wear which usually continues on the sharp ridge extending to the base of the central cusp. These elongate wear facets on the distally positioned, sharp ridges of the maxillary teeth and the mesially positioned ridges of the dentary teeth are the result of the complex jaw movement (see below). Besides these wear facets, mostly the lingual cusps of the upper teeth and the labial cusps of the lower teeth bear extensive wear. Although for some aspects a similar dental morphology can be seen in sphagesaurids (lower teeth lean anteriorly with mesiolabial carinae and the opposite on the upper teeth), here no wear facets have been reported from either the lingual surface of the maxillary teeth or the labial surface of the mandibular teeth, which is, however, very characteristic in sphagesaurids.

\section{Adductor muscle reconstruction}

Clark et al. (1989) discussed the development of some cranial adductor muscles in Malawisuchus and they inferred an adductor musculature comparable to that of extant crocodylians with somewhat more advanced pterygoid muscles (MPTD and MPTV). Malawisuchus possesses a relatively large suborbital fenestra that, indeed, refers to a developed MPTD, although the bones bordering this fenestra and providing origin surface for MPTD are not completely visible or documented. The wide lateral flanges of the pterygoid indicate a great mass of MPTV originating from here (Figure 14). This is further supported by the long and lateromedially wide angular posterior to the external mandibular fenestra. As 
seen in modern forms, these pterygoid muscles had an anterodorsal-posteroventral orientation and played an important role during jaw movement (Clarke et al. 1989, see below). The profundus part of the external adductors (MAMEP) was a reduced muscle as it is suggested by the relatively small supratemporal fossa and fenestra (Clark et al. 1989, Figure 14). Holliday and Witmer (2009) concluded that in more advanced mesoeucrocodylians (e.g. Araripesuchus, Mahajungasuchus, and Simosuchus) only one muscle (MAMEP) originates from the supratemporal fossa and the origin surfaces of the M. pseudotemporalis group are positioned onto the lateral bridge and postorbital process of the laterosphenoid. These bones cannot be very well studied, at least on the published specimens of Malawisuchus (e.g. MAL48, MAL-49, MAL-178).

The MAMP, in addition the other parts of the external adductors (MAMEM, MAMES), were at least as developed as in extant crocodylians because the quadrate-quadratojugal complex is an extended area posterior to the large, lateral temporal fenestra. Unfortunately, however, no information is available on the ventral surface of these bones (e.g. any crests or rugose surface ventrally on the quadrate) that could help in judging the extent of these adductors more accurately. Nevertheless, the strongly elongate, tall and massive post-dentary part of the mandible with a huge external madibular fenestra clearly suggests that a great mass of adductors attached in the mandibular fossa. The anteroposteriorly long surangular served as an insertion site for the MAMEM and MAMES; two short muscles that have a roughly vertical or anteroventral orientation. The external mandibular fenestra (covered internally by a fascia that served as an insertion area for MAMP; Holliday and Witmer 2007) is almost as large as the orbit suggesting a great mass of MAMP relatively to that of extant forms. It can be concluded that the adductor musculature of Malawisuchus was strongly developed (especially MPTV and perhaps MAMP) that is certainly an indicator of the specialized jaw mechanism and intraoral food processing. 
Jaw mechanism and possible phases of chewing

On the basis of the quadrate-articular joint, the suggested developed pterygoid muscles, the orientation of the teeth and the position of the wear facets, Clark et al. (1989) proposed a proal jaw movement in Malawisuchus (Figure 15). This type of mandibular movement occured in sphenodontians already in the Late Jurassic and Cretaceous (Throckmorton et al. 1981, Apesteguía and Novas 2003) but it is also present in the extant Sphenodon (Gorniak et al. 1982, Schwenk 2000), in protoroid kangaroos (Sanson 1989, Tomo et al. 2007) and in various groups of the Rodentia (Gans et al. 1978, Charles et al. 2007), for example rats (Weijs 1975) or golden hamsters (Gorniak 1977). Although the dentition of sphenodontians and rodents are completely different from each other and also from that of Malawisuchus, several characters are similar and comparison of the listed features can help in providing more details of this movement in crocodylians.

In proal movement, especially in particular phases of the powerstroke, the mandible translates anteriorly during jaw closure that is allowed by the elongate glenoid surface of the articular. In the rat, the concave articular fossa (on the skull and not on the mandible as in reptiles) is two times longer and one third wider than the mandibular condyle (Weijs 1975). Both in the rat and in sphenodontians (including the Late Jurassic Opisthias, the Early Cretaceous Toxolophosaurus [Throckmorton et al. 1981] and Sphenodon [Gorniak et al. 1982, Schwenk 2000]), the symphysis is unfused but connected by a fibrocartilagous pad (Weijs 1975) that allows a rotation of the hemi-mandibles along their long axis. In Malawisuchus, the glenoid surface is two times longer relative to that in extant crocodylians (Clark et al. 1989), however, it is not a concave fossa but rather a flat surface, not wider than the articular surface of the quadrate, and the mandibular symphysis is ossified at least in specimen MAL-49 (Gomani 1997). These features suggest some important differences in the process of proal movements 
among these groups. Weijs (1975) demonstrated that in the rat, the horizontal rotation of the hemi-mandibles about the symphysis results in a lingual component of the mainly anterior movement of the jaws during powerstroke and this enhances grinding efficiency. Indeed, this rotation can be useful in a large and roughly flat grinding surface of molars that exists in the rat (Weijs 1975:fig. 6). In Sphenodon, this rotation of the mandibles appears to be not as important as in the rat, because the teeth of Sphenodon are cuspidate and the single cusped lower tooth row is fitted between the palatal and maxillary tooth rows (Robinson 1976, Gorniak et al. 1982). In Malawisuchus, the fused symphysis (Figure 13B) and most probably the wide lateral flanges of the pterygoid did not allow any kind of rotation of the hemimandibles. It was actually not necessary because the teeth are not flat but pointed and cuspidate with straight but worn mesial and distal carinae on the main cusp of the dentary and maxillary teeth, respectively. These features of Malawisuchus rather indicate a relatively precise bite completed with proal movement of the jaws. As it was pointed out by Clark et al. (1989), wear facets on the carinae of the upper and lower multicusped molariform teeth are not due to direct tooth-tooth occlusion but most probably to the processing of food. Similarly to Sphenodon (Robinson 1976), an advanced occlusion typical in mammals or in the recently described Pakasuchus (O’Connor et al. 2010) was not present in Malawisuchus, but rather teeth came into an interdigitating position during jaw closure. On the other hand, apical wear on the main cusps and the labiolingual accessory cusps might also be the result of some accidental tooth-tooth occlusion.

Whereas in the rat all movements are bilaterally symmetrical and simultaneous chewing occurred on both sides (Weijs 1975), chewing in Sphenodon is strongly asymmetrical with frequent side switching (Gorniak et al. 1982, Schwenk 2000) despite that both groups have mobile symphysis. In Malawisuchus, symphysis is fused and no irregular abrasion of the teeth has been reported, thus a unilateral jaw movement, as seen in Sphenodon or in other 
heterodont notosuchians (e.g. sphagesaurids, see later), is not feasible. A bilaterally symmetrical food processing without rotation of the hemi-mandibles can be suggested. Nevertheless, frequent side switching during food processing in Malawisuchus cannot be excluded that might result in a similar dental wear pattern of both sides. In Sphenodon and the rat, proal movement does not necessarily occur in each cycle of mastication. For example, during fast opening of the jaws, the mandible of Sphenodon moves posteriorly and in the following fast closure it does not slide anteriorly thus no shearing but only simple crushing-puncturing of the food exists. Anterior translation (i.e. proal movement) occurs only later during the end of the mastication process in which the slow close proal powerstroke provides the „shearing phase” (Schwenk 2000:229) during which the food is completely masticated. In the rat, during the preparatory stroke of mastication, the mandibles strongly translate anteriorly during opening of the mouth to bring the incisors closer to each other (Weijs 1975). During jaw closure, forward shifting of the mandibles (approximately 2 $\mathrm{mm}$ ) occurs only in the powerstroke when the molars grind up the food.

Based on this information and comparison with the chewing cycles of Sphenodon and the rat, the following phases of food processing can be reconstructed in Malawisuchus (Figure 15): 1) in the preparatory stroke, the M. depressor mandibulae (MDM) opened the mouth and the prey was captured predominantly by the action of external adductors, but also by that of MAMP and MPT. The mode of prey capture depends on the type of food (e.g. in Sphenodon insects are captured by fast protruding of the tongue but young mice is captured by biting it with the anterior teeth, Gorniak et al. 1982). In Malawisuchus, however, the fleshy tongue must have been more similar to that of extant crocodylians than to that of the fast and protruding tongue of various lepidosaurs. Hence, it is suggested that the food was captured by biting with the premaxillary teeth and the anterior, symphyseal end of the mandibles (even if this region was edentulous). Besides the pterygoid muscles, this fast closure most probably 
was motorized mainly by the short and vertically oriented parts of MAME that are responsible for fast jaw closure (Iordansky 1964). Because the mandibles are short, it is supposed that their anterior translation during jaw opening (as seen in the rat) occured to provide a better efficiency in prey capture. When prey was captured, it was shifted posterolaterally by the tongue between the upper and lower multicusped teeth.

2) Powerstroke can be divided into a crushing-puncturing phase (Figure 15C) and a later shearing phase (Figure 15D) in Malawisuchus. The crushing-puncturing phase began with the rough crushing of the food (e.g. the hard exoskeleton of insects) into smaller pieces by the pointed main cusps of the multicusped teeth. In this early stage of the power stroke, anterior shifting (i.e. proal movement) of the mandibles may have not occured. This was followed by the shearing phase when the simple orthal jaw closure was associated with proal movement of the lower jaws. Similarly to Sphenodon (Gorniak et al. 1982), this anterior shifting of the mandibles was most probably motorized by the pterygoid muscles (Clark et al. 1989) which, in contrast to MAMP or the external adductors, have an important anterior component during acting. In this phase, due to the proal movement, the posteriorly leaning, upper multicusped teeth with their distal, sharp carina came into a shearing (but not occluding) position with the anteriorly leaning mesial carina of the lower teeth to produce more effective processing of the food (Figure 15D, E). In contrast to Sphenodon and the rat, the tip of the main cusp of the upper teeth probably did not occlude regularly with any part of the lower teeth; only accidental occlusion might have occurred with the labially positioned acessory cusps and, vice versa, the same could have happened to the lower teeth.

3) In the final stage of oral food processing, when the food was completely triturated, proal movement stopped and food was transported into the posterior part of the oral cavity to be swallowed. 
Chimaerasuchus Wu, Sues and Sun, 1995

Chimaerasuchus paradoxus Wu, Sues and Sun, 1995

(Figure 16, 17)

Holotype - IVPP V8274, fragmentary cranial material including the rostrum, an incomplete and dorsoventrally crushed mandible, one isolated molariforrn and two isolated caninifom teeth, some postcranial material. The type material is the only specimen referred to this taxon (Wu et al. 1995, Wu and Sues 1996).

Type Locality—South bank of the Yangtze River, opposite Yichang, Hubei Province, China (Wu and Sues 1996).

Type Horizon-Wulong Formation, late Early Cretaceous, Aptian-Albian (Wu et al. 1995).

\section{General cranial and mandibular morphology}

Although only the rostrum is preserved of the skull (Figure 16), it is clear that it was relatively short compared to the total skull length, as it is indicated by the length of the mandible (Wu and Sues 1996). The rostrum is wider than high, constricted at the level of the prefrontal and its lateral margin is slightly concave. The external nares are facing anteriorly as in most notosuchians. Based on a small, preserved anterior part on the left side, the orbits were facing anterolaterally as typically seen in predominantly terrestrial forms. The posterior region of the secondary bony palate with the secondary choana is not preserved. There is a slight constriction between the maxilla and premaxilla. On the thick and massive palatal part of the maxilla four, huge and shallow alveoli are present (Figure 16(B)).

The mandible of Chimaerasuchus has two unusual features. First, the dentary is mediolaterally wide with one, shallow alveolus preserved. Based on these features, the molariform teeth were presumably all wide in the lower tooth row. Second, the postdentary 
part of the mandible behind the external mandibular fenestra curves posteromedially, thus the distance is shorter between the contralateral quadrate condyles than that between the external mandibular fenestrae. This results in a mediolaterally wider mandibular adductor fossa relatively to other crocodylians. The glenoid surface of the articular is an anteroposteriorly elongate (longer than wide), slightly concave surface and no buttress is present between the glenoid surface and the retroarticular process, similarly to other notosuchians with complex jaw mechanism. The position of the glenoid surface relative the occlusion surface is unknown; nevertheless, the general morphology of the mandible and the position of the last, shallow and wide alveolus in the dentary suggest that the glenoid surface was at the same or below the level of the occlusion surface, similarly to Notosuchus, Malawisuchus or Simosuchus. The exact number of dentary teeth is unknown. However, the four, wide alveoli in the maxilla indicate four, wide functional teeth in the lower jaw too. If this is correct and dental occlusion was present as it has already been suggested (Wu et al. 1995), then the dentary possessed at least three-four wide, functional teeth that occluded with the maxillary ones. Because the small, preserved part of the mandibular symphysis almost reaches the anterior level of the last dentary alveolus, an elongate, although dorsoventrally shallow $(\mathrm{Wu}$ and Sues 1996) mandibular symphysis can be suggested.

\section{Dentition and possible extant analogues}

Although the dentition of Chimaerasuchus is poorly known, the preserved teeth and alveoli unambiguously reveal one of the most unique dental apparatus ever seen in crocodyliforms. It is unique because of the extremely reduced number of teeth in each jaw quadrant (Figure 16). In the upper jaw, Chimaerasuchus possessed at least six teeth including two procumbent premaxillary and four multicusped maxillary teeth (Figure 16, 17) (although the maxilla-jugal connection is present on the left side, the position of the last maxillary tooth is unknown). Of 
the premaxillary teeth, only the right second one is preserved in situ (Figure 17(D-G)). This tooth is conical, slightly curved distally, and it is ornamented by longitudinal enamel wrinkles but a distinct mesial or distal carina is not present. Two isolated conical teeth have been interpreted by $\mathrm{Wu}$ and Sues (1996) as the first teeth of the contralateral premaxillae (Figure 17(A, B)). These teeth are similar in morphology to the anterior tooth in the right premaxilla but it cannot be excluded that they both are or one of them is from the anterior dentary alveoli. Of the maxillary dentition, four alveoli are known from the left side and one from the right side. These alveoli and the teeth are huge relative to the size of the rostrum and they are linguolabially wider than mesiodistally long except for the first, right alveolus which is slightly longer mesiodistally. Teeth are preserved only on the left side in the second and third alveoli.

The second tooth is well preserved and the labiolingually wide, oval crown possesses three rows of cusps which are roughly parallel to the sagittal plane (Wu and Sues 1996). The labial row contains seven cusps and this row curves slightly lingually. The central row has seven cusps and the lingual one has perhaps five but it is impossible to determine precisely because this part of the crown is damaged (contra Wu and Sues 1996). The cusps curve distally and their size decreases distally. All cusps bear a distal, unserrated carina and except for the first, largest cusps all the cusps behind these have a weak mesial carina (contra Wu and Sues 1996). The cusps have a smooth and relatively thick enamel cover.

The occlusal surface of the third tooth is completely broken thus the individual cusps cannot be observed (Figure 17(E, F)). Nevertheless, the main tooth morphology can be seen. Similarly to the second tooth, the central row of cusps could have been the most prominent here. As it was noted by Wu and Sues (1996), this third tooth is more laterally positioned than the second one, thus the labial row of cusps of the second tooth is in one line with the central 
row of the third tooth. The fourth maxillary alveolus is in a more lateral position compared to the third one but not as much as the third one relative to the second one.

Only a shallow depression is preserved on the posterior part of the right dentary that was interpreted as an alveolus (Wu and Sues 1996). The type material contains a fragmentary bone with a single, multicusped tooth that is similar in morphology to the multicusped tooth preserved in the left maxilla (Figure 17(C)). Wu and Sues (1996) mentioned this tooth but no details of this specimen was described or figured. However, this tooth, although slightly broken and apparently eroded occlusally, bears several important features related to the feeding mechanism of Chimaerasuchus. The tooth is sitting in an alveolus, but it is not clear if the bone fragment bearing this tooth represents a maxilla or a dentary. Furthermore, it is also uncertain if this specimen belongs to the same individual as the skull and mandible remains or not. Another piece of bone fragment is connected to this bone which, in case the latter is a maxilla, would be the palatine. This „maxilla hypothesis” is more reasonable because the connecting bone is too robust to be the splenial in case it was a dentary. If it indeed represents a fragment of a maxilla, based on the size of the cusps, the tooth is from the posterior part of the right maxilla. The number of cusps in this tooth is $5 ?-7-7$ where the shortest, broken row (maybe the labial) contains about five cusps. The cusps of the central row appear to be broken but their base can be observed. The mesially positioned four largest cusps of the supposed lingual row, however, show a smooth occlusal facet including the EDI. I suggest that the latter is indeed an extensive wear facet and not a damaged part of the tooth (Figure 17(C)). Dentition similar in some aspects to the multicusped teeth of Chimaerasuchus occurs in tritylodontid synapsids as it was pointed out by Wu and Sues (1996). In addition, various groups of primitive mammals (e.g. the allotherian Eleutherodon, Butler 2000, multituberculates, Krause 1982, Gambaryan and Kielan-Jaworowska 1995) possess multicusped teeth with mesiodistally aligned rows of cusps that received the rows of the lower 
teeth during palinal and orthal jaw movements. Among extant reptiles, however, the only form that possesses similar dentition and jaw function to those of Chimaerasuchus is Sphenodon (see below).

\section{Dental wear}

No extensive wear can be observed on the anterior, conical tooth preserved in the right premaxilla and on the isolated conical teeth referred to Chimaerasuchus. Wu and Sues (1996) reported that the in situ multicusped tooth in the left maxilla does not bear wear facets.

Detailed SEM studies, however, revealed a small, but informative wear facet on the tip of the mesial, largest cusp of the labial row (Figure 17(H-J)). This subhorizontal, slightly lingually facing circular wear facet (labiolingual diameter $0.6 \mathrm{~mm}$ ) is gouged only in the enamel and not the dentine, thus no EDI can be examined. Numerous shallow and some deeper, parallel scratches present on this flat wear facet are roughly parallel with the sagittal plane. Besides the scratches, some shallow, irregular areas and pits also occur on this worn surface. The other cusps of this crown are either complete or crushed thus no wear facets can be observed. Unfortunately, no SEM studies are available of the worn four mesial cusps that possibly were part of the lingual row of the isolated multicusped tooth referred to Chimaerasuchus. This wear facet is smooth and the worn enamel and dentine surfaces are evenly eroded in numerous parts (Figure 17C). The worn dentine surface is ornamented by a few, heavy pits. This tooth was in a more erupted position and became more heavily worn than the one in the skull fragment.

\section{Adductor muscle reconstruction}

For the gross interpretation of the cranial adductor muscles of Chimaerasuchus only the mandible can be used. The extended and smooth posterolateral and posteroventral surface of 
the angular (starting from the level of the posterior end of the external mandibular fenestra) indicates a relatively large insertion area for the MPTV that could have been well-developed and perhaps played an important role in the suggested complex jaw mechanism (Wu et al. 1995). The MAMP, which attaches among others on the internal surface of the fascia covering the external mandibular fenestra, might have been an important but perhaps not a highly developed muscle. Unfortunately, the surangular is unknown, thus the insertion areas of MAMES and MAMEM cannot be studied.

\section{Jaw mechanism}

Although the remains of Chimaerasuchus are very fragmentary and the dentition is very poorly known, several aspects of the jaw mechanism can be reconstructed. On the basis of the anteroposteriorly elongate glenoid surface, the absence of a buttress behind the glenoid surface, and the morphology of the in situ preserved, multicusped tooth, Wu et al. (1995) and Wu and Sues (1996) reconstructed proal movement in Chimaerasuchus. This is most probably true but the results of the wear pattern analysis i.e. the parallel scratches present on the described wear facet testify only the anteroposterior component of the mandibular movement. Details of the EDI and the orientation of scratches on vertical or subvertical wear facets (as in sphagesaurids) would be more informative in determining the direction of jaw movement. However, the roughly horizontal wear facet on the tooth preserved in the rostrum extended only on the enamel and the details of wear of the isolated tooth are unknown. As it was pointed out by Krause (1982) in multituberculate mammals and was applied by Wu and Sues (1996) to Chimaerasuchus, cusp orientation and the curvature of cutting edges of cusps can help to reconstruct the occlusal pattern and the direction of mandibular movement. Indeed, these features can be useful, but reconstruction of jaw mechanism is not always unambiguous, because disctinct wear pattern with parallel scratches, the most direct evidence of occlusal 
pattern and/or food processing, does not always lead to the same conclusion. For example, in the sphagesaurid Armadillosuchus, tooth orientation (distally oriented upper and mesially oriented lower teeth anteriorly) is similar to that of Malawisuchus and Notosuchus, but the position and orientation of wear facets and the direction of scratches are completely different indicating different jaw mechanisms (see below).

The distally curved cusps of the multicusped tooth and the distal carina on the cusps of Chimaerasuchus suggest that the supposed lower multicusped teeth with opposite cusp configuration came into occlusion with the upper ones during an anterior shifting of the mandibles, i.e. in a proal phase, roughly similar to Sphenodon (Wu and Sues 1996). However, there is an important difference between the tooth contact of Sphenodon and that of Chimaerasuchus: the acrodont teeth of Sphenodon are not multicusped, but they are in two rows in the upper jaw and one tooth row is in the dentary, thus an interdigitation of tooth rows but no dental occlusion occurs here (Gorniak et al. 1982). In Chimaerasuchus, however, this interdigitation was most probably present between the mesially and distally aligned rows of cusps where the tip of the cusps occluded with the concave enamel surface of the crown to work in a manner of shearing contact. Interestingly, however, no mesiodistally oriented scratches have been found on the concave enamel surface between the rows, although it is important to note that this part of the crown is slightly crushed and actually wear pattern is not really extensive on this tooth.

To sum up, the orthal jaw closure of Chimaerasuchus was clearly associated with an anteroposterior component of the mandibular movement. This movement was most probably proal, but additional cranial material is essential to confirm this notion. The developed MPTV indicated by the extensive insertion area on the angular would further support the proal hypothesis because in Sphenodon, the advanced pterygoid muscles pull the mandibles forward during jaw closure. These features along with wear facets on the two multicusped teeth 
unambiguously indicate a precise occlusion and effective oral food processing in Chimaerasuchus.

Notosuchus Woodward, 1896

Notosuchus terrestris Woodward, 1896

(Figure 18, 19)

Lectotype-MLP 64-IV-16-5, skull and jaw (Gasparini 1971).

Referred specimens-MACN-Pv-RN-1037, MPCA-Pv-789, MUCPv-137, MUCPv-147, MUCPv-149, MUCPv-198 (cranial and postcranial material), MACN-Pv-N-43, MLP-64-IV16-7, MLP-64-IV-16-8, MLP-64-IV-16-24 (subadult specimens), MACN-Pv-RN-1046, MACN-Pv-RN-1048, MACN-Pv-N-24, MACN-Pv-N-43, MACNPv-RN-1038, MACN-PvRN-1039, MACNPv-RN-1040, MLP-64-IV-16-6, MLP-64-IV-16-11, MLP-64-IV-16-15, MLP-64-IV-16-16, MLP-64-IV-16-23, MLP-64-IV-16-24, MACN-Pv-N-23, MACN-Pv-N107, MACNPv-RN-1046, MACN-Pv-RN-1048, MACNPv-RN-1118, MACN-Pv-RN-1119, MLP-64-IV-16-1, MLP-64-IV-16-2, MLP-64-IV-16-7, MLP-64-IV-16-8, MLP-64-IV-16-31, MPCA-Pv-789/1, MPCA-Pv-791, MACN-Pv-RN-1037, MACN-Pv-RN-1041, MACN-PvRN-1045, MACN-Pv-RN-1127, MLP-64-IV-16-3, MLP-64-IV-16-5, MUCPv-35 (complete or partial skulls) (Gasparini 1971, Andrade and Bertini 2008b, Fiorelli and Calvo 2008, Lecuona and Pol 2008).

Type Locality-Neuquén Basin, Argentina, South America (Fiorelli and Calvo 2008) Type Horizon-Bajo de la Carpa Formation, Río Colorado Subgroup, Neuquén Group, Late Cretaceous, Santonian (Leanza et al. 2004).

Remarks-Notosuchus terrestris is one of the earliest heterodont crocodyliforms ever discovered. Already Woodward (1896) noted the peculiarities of this animal and later on, 
Price (1959), Gaparini (1971) and others discussed the cranial, dental (e.g. Andrade and Bertini 2008b, Fiorelli and Calvo 2008, Lecuona and Pol 2008) and postcranial features (Pol 2005, Fiorelli and Calvo 2008) of this form. They mentioned heterodonty, wear facets, and proal movement but none of these studies deal with the detailed functional morphological background of jaw movement and with that of the dental fuction in Notosuchus.

\section{General cranial and mandibular morphology}

Notosuchus has a short and massive skull with huge anterolaterally facing orbits and large, triangular lateral temporal fenestra bordered laterally by a long and massive lower temporal bar. The high and extremly short rostrum (Andrade and Bertini 2008b) becomes narrower anteriorly ending in a rostrally oriented, confluent narial opening. Small antorbital fenestrae are present. The quadrates are relatively short, their medial and lateral condyles are well separated by a wide notch and they are oriented posteroventrally. The supratemporal fenestra is anteroposteriorly elongated, narrow and together with the supratemporal fossa it is relatively small (Figure 18(A)). The shelf of the fenestra extends posteriorly rather than lateromedially (Fiorelli and Calvo 2008). All these basic features are similarly present in Malawisuchus. An important difference is that the skull of Notosuchus is generally more than two times larger than the skull of Malawisuchus. The pterygoids are massive with wide and robust lateral flanges and contrast to Malawishuchus, the pterygoid of Notosuchus has prominent posterolaterally oriented quadrate processes (Figure 18(D)). Similarly to the protosuchian Kayenta form, this surface might have played an important role as the origin surface for jaw adductors. Based on specimens RN 1037 and RN 1041, the space between the lateral flange of the pterygoid and the mandibular rami must have been narrow (3-4 $\mathrm{mm}$ on the specimens) similarly to extant forms preventing any significant lateromedial movement. The relatively large suborbital fenestrae are anterolateral to the enormous secondary choana. 
Lateromedially on the bony palate, an additional opening is present between the maxilla and palatine of Notosuchus (maxillopalatine fenestra of Andrade and Bertini [2008b] and maxilopalatal fenestra of Fiorelli and Calvo [2008]). A similar opening, though relatively smaller, is present only in the Brazilian Late Cretaceous notosuchian Mariliasuchus, and this feature has been listed by Andrade and Bertini (2008b) among the shared characteristics of the two taxa. Important to note, however, that similar, additional cranial opening besides the 'traditional' ones (i.e. external nares, antorbital, suborbital, lateral temporal, supratemporal fenestrae, choana, incisive foramina, orbits) frequently occur among different groups of archosaurs. For example, both Araripesuchus gomesii and Uruguaysuchus aznarezi possess a small fenestra laterally between the premaxilla and maxilla (Witmer 1997) that is also present in various dinosaurs. An oval fenestra occurs palatally, posterior to the incisive foramen between the premaxilla and maxilla in the pterosaur Dorygnathus and Tapejara (Ösi et al. 2010). The function of these generally small openings are unknown, although in the latter example the opening for the vomeronasal or Jacobson's organ (as in various lepidosaurs) has been suggested. In Crocodylus embryos, there is evidence for the presense of the vomeronasal organ (e.g. Meek 1893; Parsons 1970) but in later ontogenetic stages there is no aperture for this sensory organ in the secondary palate. The maxilla-palatine fenestrae of Notosuchus (and also that of Mariliasuchus) might have had the same function as the fenestrae between premaxilla-maxilla of pterosaurs and it cannot be excluded that in such notosuchians these openings also served as the passage for the vomeronasal organ into the oral cavity. The mandible of Notosuchus is massive with an unusually long post-dentary part (the postdentary part is almost two times longer than the toothed dentary, (Figure 18(C)), similarly to Malawisuchus. Additional similarity between the two taxa is that the symphysis is not as strongly fused as in Mariliasuchus or Armadillosuchus but rather unfused as seen in the rat or in sphenodontians (Gorniak et al. 1982, Schwenk 2000). In the specimen RN 1039, the two 
dentaries do not connect with each other and the symphyseal region reaches the level of the third mandibular alveoli. The external mandibular fenestra is dorsoventrally narrow but unusually elongate. Notosuchus possesses an anteroposteriorly elongate glenoid surface that is slightly convex with an anteroposterior ridge that fits into the intercondylar notch of the quadrate (Figure 18(A)). The glenoid surface is approximately two times longer than the articular surface of the quadrate and no buttress is present at its posterior end, similarly to Malawisuchus. The glenoid surface is bordered laterally by a high margin of the surangular preventing any lateral translation of the mandible.

\section{Dentition and possible extant analogues}

Various authors working on the cranial morphology of Notosuchus provided description of the teeth (e.g. Woodward 1896, Gasparini 1971, Andrade and Bertini 2008b, Fiorelli and Calvo 2008) but due to mostly poorly preserved dental material some aspects (e.g. number of teeth, denticles on the carinae, dental wear) were still dubious. Lecuona and Pol (2008) provided the most detailed study on the dentition of Notosuchus including the morphology of wear facets, thus this study mainly follows their work completed with some personal observations. Anteriorly, the upper tooth row of Notosuchus possesses three incisiviform teeth and one enlarged caniniform tooth. These teeth are conical, straight and their enamel cover is smooth (Figure 18(E)). Neither carina nor any compressed, blade-like margin is present in these teeth. The caniniform in the premaxilla is the largest tooth in the series with conical, slightly distally, linguodistally curving shape. It does not bear any enamel wrinkles or keel distally. Lecuona and Pol (2008) reported a „transitional tooth” posterior to the caniniform tooth. This tooth is small, gracile, and bears a very weak carina like the molariform teeth. Anteriorly, the mandibular dentition starts with three incisiviform teeth that are conical, slightly distally recurved. Dentary tooth row does not possess a caniniform as the upper tooth 
row, but the incisiviforms are followed by a tringular tooth with a mesial carina. These incisiviform teeth have a forward inclination with an angle about $45^{\circ}$ (Fiorelli and Calvo 2008).

These anterior teeth are followed by characteristic molariform teeth with obliquely orientated carina. Based on the description of Lecuona and Pol (2008), in the maxilla, there are six carinate molariform teeth posterior to the transitional tooth (Figure 18(E, F)). In lateral view, these teeth are triangular, pointed ventrally, and the carina is linguodistally oriented extending from the apex to the base of the crown. The teeth changed shape during ontogeny, because in subadult specimens these molariform teeth are more bulbous (Lecuona and Pol 2008). The enamel is generally smooth, very thin and does not bear any wrinkles or rough ornamentation, as for example in Mariliasuchus. The dentary possesses six molariform teeth that are generally similar and show the opposite configuration of the carinae and orientation: here the obliquely positioned carinae are mesiolabially oriented. Lecuona and Pol (2008) reported that in some dentary teeth the carinae bear serrations that are poorly preserved.

Similarly to the crocodyliforms discussed above, Notosuchus also had a dental morphology that is unique among vertebrates (especially features of the posterior molariform teeth). The most characteristic feature of the roughly triangular, conical, molariform teeth is the obliquely oriented carina on both the upper and lower teeth (Figure 18(F)). Although Sphenodon exhibits different tooth morphology, its mandibular teeth are also anteriorly directed and they bear a sharp edge lingually that plays an important role in shearing the food during proal movements (Gorniak et al. 1982). The role of these carinae both in Sphenodon and Notosuchus are similar (see below), however, the orientation of them is clearly lingual in Sphenodon and oblique in Notosuchus. In most lepidosaurs, the carina or any kind of cutting edge frequently connecting cusps on the tooth crown is positioned occlusally or mesiodistally, or, as seen in some bicusped forms, it is transversely oriented. This is most probably related to 
the fact that proal movement characteristic for sphenodontians, some rodents and apparently in some fossil crocodyliforms does not occur in non-sphenodontian lepidosaurs where jaw closure is dominantly simply orthal.

\section{Dental wear}

Due to the generally poor preservation of the dentition of Notosuchus, the documentation of wear pattern was a difficult task to fulfill. Based on some new specimens, Lecuona and Pol (2008) provided a short description of wear facets on the teeth of Notosuchus, although those of the anterior teeth are still unknown. The caniniform premaxillary teeth bear some apical wear and randomly oriented scratches occur on its lingual surface. The following transitional tooth already possesses a weak carina posteriorly with distolingually facing, oblique apical wear facet and randomly oriented scratches lingually (Lecuona and Pol 2008). The upper molariform teeth have extensive wear facets that extend along the carina distolingually from the apex to the base of the crown (Figure 19(A)). The crown of the lower teeth are inversely oriented relative to the upper one and the complementary wear facets are along the labial surface of the mesiolabially positioned carina and extend from the apex to the base of the crown (Figure 19(B)). These wear facets of both the upper and lower molariform teeth are subparallel to the sagittal plane. The carina is always involved in these wear facets which is an elemental difference compared to Mariliasuchus where the wear facets are on the labial or lingual sides of the crown. Both the tooth crowns and the wear facets are generally in a bad condition and the enamel is extremely thin, thus the mapping of pits and scratches is complicated and the morphology of the EDI is not clear. On the basis of the figures of Lecuona and Pol (2008), some heavy scratches are vertical to subvertical mostly with random orientation, but mesiodistal scratches cannot be observed. In a more advanced stage of wear, 
these apicobasally extended, long wear facets on the molariform teeth are wider apically and the apical region becomes strongly eroded which suggests a frequent apex-apex occlusion.

\section{Adductor muscle reconstruction}

Concerning the cranial adductor/abductor musculature of Notosuchus terrestris, only Fiorelli and Calvo (2008) mentioned elongated M. depressor mandibulae attaching deeply on the posteromedioventrally facing, concave retroarticular process. Furthermore, they emphasized the development of pterygoid muscles which is further supported by my observations. In general, it can be concluded that the massive architecture of the skull and the unusually long postdentary part of the mandible with a huge, anteroposteriorly elongate external madibular fenestra indicate a great mass of attaching cranial adductors. The pterygoid flanges are wide and massive, and although they appear to be not as extended posteroventrally as in modern forms, they suggest well developed MPTV that originates from the pterygoid wings and probably from the extended posterior process of the pterygoid too (Figure 18(D)). This is supported by the massive and anteroposteriorly elongate posterior part of the angular where the attachment area of MPTV is enlarged by the mediolaterally wide ventral surface of the angular and the retroarticular process. The relatively large suborbital fenestrae and the expanded dorsolateral wall of the secondary choana formed by the medial part of the ectopterygoid and palatine indicate a developed MPTD in Notosuchus.

The ventral surface of the elongate quadrate is smooth and apparently no crests (any crests noted by Iordansky [1964] or protuberances as in hyleaochampsids [Ösi et al. 2007]) can be observed. This indicates that the relatively short and slightly anterolaterally oriented bundles of MAMP were not particularly advanced and could have been similar to those of extant crocodylians (Iordansky 1964, Holliday and Witmer 2007). Based on the relative size of the supratemporal fenestra and supratemporal fossa, the MAMEP originating from this region 
was not as reduced as in Malawisuchus (Figure 18(A)). In Notosuchus the morphology of the laterosphenoid is not clear due to the state of the materials thus the origin sufaces of MPSS cannot be studied. Other parts of the external adductors (MAMEM, MAMES) were relatively at least as developed as in extant crocodylians becasue the elongate and mediolaterally wide (approximately $5 \mathrm{~mm}$ in $\mathrm{RN} 1040$ ) surangular indicates important attachment areas for these adductors. Otherwise, the bundles of these muscles, originating from the qudratequadratojugal complex had a remarkable anterior component that along with the advanced MDM might have played an important role during the assumed anteroposterior movement of the mandibles (see below).

\section{Jaw mechanism and possible phases of chewing}

Various authors (e.g. Clark et al. 1989, Bonaparte 1991, Wu and Sues 1996, Andrade and Bertini 2008b, Fiorelli and Calvo 2008) suggested anteroposterior mandibular movement in Notosuchus terrestris. These authors based their hypothesis on the morphology of the quadrate-articular joint (anteroposteriorly elongate glenoid surface with longitudinally oriented ridge leading the quadrate condyles, lack of buttress behind the glenoid surface) and the extensive wear facets present especially on the molariform teeth. On the basis of tooth morphology and position of the wear facets, Lecuona and Pol (2008) also raised the possibility of an anteroposterior movement of the mandibles but due to the lack of numerous striae on these worn surfaces they could not find substantial evidence for that. What could have been the degree of this anteroposterior component of jaw closure, if there was any? And if there was indeed an anteroposterior component in the movement of the mandibles during jaw closure, was this a proal movement as in Malawisuchus and Chimaerasuchus or rather palinal as in other South American hetrodont notosuchians (see below)? To answer these questions, acquiring information on the details of wear pattern of the teeth would be essential. 
Unfortunately, the poor preservation of the teeth, the hardly recognizable EDI, and the basically unknown number and orientation of scratches nearly preclude the accurate reconstruction of jaw mechanism (as noted also by Lecuona and Pol 2008). The only features that can be used to infer the exact phases of jaw movement are the dental morphology and the position of wear facets. Similarly to Malawisuchus, the carina on the maxillary teeth of Notosuchus is situated on the distal side (linguodistally) of the slightly posteriorly curved crown and importantly, no carina is present mesially. The lower molariform teeth are complements of the upper ones by having mesiolabially positioned carina (Figure 19(A, B)). The wear facets along the carina are lingually positioned on the upper teeth and labially on the lower ones, and they are subparallel to the sagittal plane. This had led Lecuona and Pol (2008:415) to conclude that there was a ,precise tooth-tooth contact during occlusion”. Palinal movement (i.e. the mandibles are pulled backwards during jaw closure) can be exclued in Notosuchus because of the position of the carinae and the position of wear facets: in a backward movement of the mandible the lingual surface of upper tooth and the labial surface of the lower one could not come into a functionally effective shearing contact (Figure 19(A-C)). Reconstruction of the upper and lower tooth rows in a position of closed mandibles clearly indicates that the complementary wear facets (with a mesiodistal length of max. 2-3 $\mathrm{mm}$ ) roughly subparallel with the sagittal plane could have been abraded only if the lower jaw moved orthally (Figure 19(D)) and/or anteriorly (Figure 19(E)) in the last phase of jaw closure. This movement brought the carinae of the lower teeth into an antagonistic contact with those of the upper teeth and it means that a proal movement might have existed during food processing. Another explanation for the wear facets would be the simple orthal jaw closure in which the lingual surface of the upper and the labial surface of the lower teeth occlude with each other in a kind of precise shearing contact as it was demonstrated in some pterosaurs and ankylosaurs (Ösi 2011). The efficiency of this movement during food 
processing is evidenced by some heavy, approximately vertically oriented scratches (see Lecuona and Pol 2008: fig. 6B, C). In this case, regular occlusion during orthal jaw closure in a crocodyliform with strongly elongated jaw articulation would probably have resulted in a heavy apical wear. This is supported by some teeth illustrated by Lecuona and Pol (2008: fig. $6 \mathrm{G}, \mathrm{I})$ that possess a strongly worn apical region of the crown. Allowing for the currently available evidences only orthal jaw closure with preceize dental occlusion can be inferred in Notosuchus with high confidence. If proal shift of the mandibles had also occurred, which notion is supported by the presence of anteroposteriorly elongate mandibular glenoid surface, it could have produced such wear facets too. However, the lack of numerous, mesiodistally oriented, parallel scratches on the wear facets that would be good indicators of anteroposterior movement may suggest that this anterior component was not present or it was not as remarkable as in Malawisuchus or in Sphenodon. It should be also emphasized that only a few teeth are available to demonstrate nicely preserved dental wear (Lecuona and Pol 2008), thus, maybe in the future with additional, well preserved dental material (including EDI), the presence or absence and the degree of proal movement could be testified.

In case only orthal jaw movement was performed (Figure 19(D)), the phases of chewing were restricted to very simple, dorsoventral rotation of the lower jaws. The food item was caught by the anterior teeth and crused and chewed by the posterior carinate teeth. Reckoning with proal mowement too, the phases would have been similar to those reconstructed in Malawisuchus with the pterygoid muscles pulling the mandibles forward when the jaws were being closed. MDM and perhaps MAMES and MAMEM (the latter two with important anteroposteriorly oriented bundles in Notosuchus) could have been responsible for the backward sliding of the mandibles in the opening phase. 


\section{Heterodont notosuchians with palinal jaw movement}

Systematic Palaeontology

Mesoeucrocodylia Whetstone and Whybrow, 1983

Notosuchia Gasparini, 1971

Mariliasuchus Carvalho and Bertini, 1999

Mariliasuchus amarali Carvalho and Bertini, 1999

(Figure 20-25)

Holotype - UFRJ DG 50-R, partially complete skeleton with a nearly complete skull and mandible (most probably represent a juvenile or subadult animal).

Referred specimens-MN 6756-V, MZSP-PV-50, MZSP-PV-51, UFRJ-DG-106-R, URC R

67, URC R 69 (cranial and postcranial material), MN 6298-V, UFRJ-DG-105-R, UFRJ-DG-

56-R, URC R 68, (cranial material) (after Andrade and Bertini 2008c).

Type Locality - Close to the city of Marília, São Paulo State, southeastern part of the Bauru Basin, Brazil (Andrade and Bertini 2008c).

Type Horizon - Upper Cretaceous, Adamantina Formation (or Araçatuba/Adamantina Formations), Bauru Group. The stratigraphical context and the age of the different formations of the Bauru Group are still controversial in various aspects (Andrade and Bertini 2008b, c). Whereas Zaher et al. (2006) assign the upper part of the Adamantina Formation as the locality of Mariliasuchus specimens, Andrade and Bertini (2008c) considered the type-horizon of Mariliasuchus as the Araçatuba/Adamantina formations rather than the upper Adamantina column. Both teams (and references therein) agree in a Campanian-Maastrichtian age for the Mariliasuchus material (Andrade and Bertini 2008b, c). However, Fernandes and Coimbra 
(1996) considered the Adamantina Formation to be the oldest part of the Bauru Group with a Turonian-Santonian age. Candeiro et al. (2006) agrees with that and explains this hypothesis with a Campanian depositional hiatus. So, up to the present, it is unclear if Mariliasuchus (and also Adamantinasuchus, Armadillosuchus and other sphagesaurids) are early Late Cretaceous (Turonian-Santonian) or younger (Campanian-Maastrichtian).

Remarks-There is no doubt that the cranial material of Mariliasuchus amarali represents one of the best preserved remains of heterodont notosuchians (Carvalho and Bertini 1999, Vasconcellos and Carvalho 2005, Zaher et al. 2006, Andrade and Bertini 2008b, c, Nobre et al. 2008) with complex food processing. Several complete specimens are three-dimensionally preserved with complete dental series showing beautiful wear patterns. This assemblage provides a good chance to understand the jaw mechanism and dental function of this peculiar animal.

Nobre et al. (2007) described another species of Mariliasuchus, M. robustus. This species is repesented by a partially preserved skull with articulated, fragmentary mandibles and other, non-articulated skull fragments, postcranial remains and an isolated caniniform tooth (UFRJDG 56-R). Although among other features the general robustness of the cranial bones and the highly elevated postdentary part of the mandible appear to support the validity of this taxon, the lack of the posterior part of the skull and mandibles and their tight articulation prevent the detailed study of the temporal region, origin and insertion surfaces of adductor muscles and the dental features.

\section{General cranial and mandibular morphology}

The skull of Mariliasuchus amarali is extremely short compared to skull width. The narrow rostrum is slightly wider than high and the confluent external nares are facing anteriorly (Figure 20(B)). At the anterior part of the orbits, the rostrum becomes rapidly narrow and 
constricted. Here on the lateral side, at the anterior end of the massive lower temporal bar, the skull is slightly wider than more posteriorly. Almost all openings in the skull of Mariliasuchus are huge including the anterodorsolaterally facing circular orbits, the huge trapezoid supratemporal fenestrae that occupy the greatest part of the skull roof and the anteroposteriorly long lateral temporal fenestrae. The unusually extensive supratemporal fenestrae almost reach the posterior margin of the skull roof, whereupon the parietal becomes narrower than the frontal. The shelf-like part of the supratemporal fenestrae is three times larger than the area of the supratemporal fossa (Figure 20(A)). The whole postorbital is thin and rather bar-like. The posterior margin of the skull is a straight and massive surface with some shallow crests and ruguse surfaces for the attachment of Musculus depressor mandibulae (MDM) and some neck muscles. The quadrates are short only slightly overhanging the posterior margin of the skull roof and they are ventrally, posteroventrally directed. The quadrate condyles are separated by a wide and developed sulchus. Whereas the lateral condyle is lateromedially wide and almost horizontal, the medial condyle is pointed and projects posteroventrally. The occipital condyle has a posteroventral orientation as frequently seen in notosuchians. The bony palate is perforated anteriorly by the confluent incisive foramina. Posteriorly, at the level of the penultimate maxillary teeth, two small (maximum length 5-6 $\mathrm{mm}$ ), bean-shaped openings are present between the maxillae and the palatines which appear to be morphologically similar and perhaps had the same function as the slightly larger, maxilla-palatine fenestrae of Notosuchus (Andrade and Bertini 2008b, Figure 20(D), see above). Posterolateral to these openings are the large, oval suborbital fenestrae bordered laterally by the robust ectopterygoids, medially by the palatines and anteriorly by the maxillae. The choanae bordered anteriorly by the palatines are huge triangular openings separated at least dorsally by a narrow, bony septum. Posterior to the choanae are the relatively small and unusually week pterygoids $45^{\circ}$ offset from the horizontal 
plane. The pterygoids of Mariliasuchus markedly differ from those of most mesoeucrocodylians (also from Notosuchus) in the strongly reduced, lateral pterygoid flange (Figure 20(D, E)). The pterygoids possess posterolaterally oriented quadrate processes similarly to that of Notosuchus revealing a broad contact with the quadrates. These posterior lobes of the pterygoids are not as developed as in the protosuchian Kayenta form (Clark 1986). When the mandibles are closed, the distance between the pterygoid-ectopterygoid wing and the ipsilateral mandibular ramus is about $5 \mathrm{~mm}$ which might have allowed some lateromedial displacement of the mandibles.

The mandible is massive with relatively long postdentary part (approximately $60 \%$ of the total length). In contrast to Notosuchus, Malawisuchus (Gomani 1997) and Chimaerasuchus, the symphysis of Mariliasuchus is strongly fused up to the level of the sixth-seventh dentary teeth (Figure 20(H)). In dorsal view, posterior to the last alveolus, at the dentary-surangular connection, the mandible is very massive and wide. The oval-shaped external mandibular fenestra is much smaller relative to that of Notosuchus and it is positioned far anteriorly. Another important difference compared to Notosuchus is that the mandibular glenoid surface is slightly above the occlusal plane in Mariliasuchus. Otherwise the glenoid surface is anteroposteriorly elongate and convex both anteroposteriorly and mediolaterally. The glenoid surface is approximately 1.5 times longer than the condyles of the quadrate (Andrade and Bertini 2008a) and no buttress is present at its posterior end, similarly to other heterodont notosuchians. The high, posterior part of the surangular, however, prevents the significant lateral sliding of the mandibles. The wide and slightly concave retroarticular process is facing posterolaterodorsally as in Notosuchus. 
The dentition of Mariliasuchus is heterodont but none of the teeth are multicusped (Andrade and Bertini 2008c, Figure 21). The three conical teeth in the premaxilla are strongly procumbent. Among the premaxillary teeth, the first tooth is the smallest and it is almost straight. The second tooth is slightly larger and moderately curved distolabially in specimen MZSP-PV 51. At least in one specimen, MN 6756, this second tooth had just erupted and bears unserrated carinae labiolingually and its enamel surface has very fine longitudinal wrinkles. The third premaxillary tooth is the largest in the whole tooth row. It is a caniniform tooth ornamented by longitudinal enamel wrinkles and has a slightly distal curvature at the apical end of the crown (Figure 21(A, G-I)). On the left caniniform of MN 6756, a weak mesial or linguomesial carina can be seen. These longitudinal enamel wrinkles on the premaxillary teeth of MZSP-PV 51 are not as marked as on MZSP-PV 50 or MN 6756. Behind this massive tooth is a transitional one the alveolus of which is between the premaxilla and maxilla. This conical and distally curving tooth is smaller than the first premaxillary tooth and it has fine longitudinal wrinkles but still no mesiodistal carinae.

The anterior teeth of the lower jaw are even more procumbent (almost horizontal) than the first two premaxillary teeth (Figure 21(B), 22(D)). They are conical and the first dentary tooth is longer and more robust than the second one. This tooth is well-preserved in UFRJ 105-R and 106- $\mathrm{R}$ and it bears longitudinal enamel wrinkles. The second dentary tooth is smaller than the first one and apparently a weak carina is present mesiodistally. Although not with as low angle as the first two dentary teeth, the third tooth is still procumbent. It is slightly smaller than the second one and has a conical shape with fine longitudinal enamel wrinkles. In the left dentary of specimen UFRJ 105-R, this tooth is just erupted and complete and a welldeveloped, roughly serrated distal carina can be observed.

Distal to the transitional tooth between the premaxilla-maxilla, five stocky and carinate teeth occur in the maxilla (Figure 21). This part of the tooth row is slightly divergent distally but 
the orientation of the serrated carinae is always roughly parallel with the sagittal plane, so the carinae are not obliquely oriented as seen in Notosuchus. The serration of the carinae is „composed of isolated tuberous anisomorphic true denticles” (Andrade and Bertini 2008c: 63, Figure 21(C-F)). The first tooth behind the transitional one is smaller than the transitional tooth and its apical angle is about $60^{\circ}$. On this tooth, the distal carina is more advanced than the mesial one. The tooth crown is ornamented by fine, approximately longitudinal enamel ridges. The second tooth is one of the largest among these distal teeth. Whereas its mesial carina is strongly convex, the distal carina is almost straight. These teeth are not really pointed and the apical angle is about $90^{\circ}-110^{\circ}$. Basically the irregular enamel wrinkles become longitudinal rows of small tubercles and this is characteristic of the following teeth too that resemble this second tooth in every aspects. The last, sixth maxillary tooth is two times smaller than the penultimate tooth but otherwise it is very similar to the previous ones except for the development of basal tubercles (Figure 21(G, H)).

Posterior to the third, slightly procumbent tooth, the dentary teeth are similar to the posterior five teeth of the maxilla. The fourth dentary tooth is the largest. It is not as stocky as the more posterior ones (apical angle is still close to $45^{\circ}$ ), but it bears already serrated carinae mesiodistally. The fifth to eighth dentary teeth are similar in size and morphology, although the eighth tooth is slightly smaller than the fifth to seventh (Figure 20(B, D)). As in the case of the posterior maxillary teeth, the posterior part of the dentary tooth row is also divergent laterally and the carinae of the lower teeth are aligned mesiodistally. The last, $9^{\text {th }}$ dentary tooth is one of the smallest in the tooth row.

One of the most characteristic features of the posterior dentition of Mariliasuchus is the mesiodistally oriented carinae serrated with relatively stocky tubercles and the complex, labiolingual ornamentation (Figure 21(C, E)). This type of carina differs from the blade-like serration of ziphodont forms (Andrade and Bertini 2008c). Various herbivorous dinosaurs 
(e.g. basal ornithischians, pachycephalosaurs [Barrett 2000], thyreophorans [Vickaryous et al. 2004]) possess carinate teeth with cuspidation but their teeth are more compressed labiolingually and the carinae are composed of more pointed denticles. Some of these dinosaurian groups (e.g. ankylosaurs) have a cingulum at the basis of the teeth but none of them have a so complex ornamentation (i.e. wrinkles, small tubercles in longitudinal rows) on the labiolingual surfaces as those of the posterior teeth of Mariliasuchus. Extant animals with teeth similar in most aspects to those of Mariliasuchus do not exist. Various lizards (e.g. scincids, teids) possess relatively massive, stocky teeth ornamented by longitudinal enamel

wrinkles. Howerer, these teeth do not have mesiodistally positioned, cuspidate carinae. Some iguanid, mostly herbivorous lizards (e.g. Iguana, Cyclura, see Ösi 2011) have teeth with cuspidate, mesiodistally oriented carinae but these tooth crowns have a smooth labiolingual surface and the tooth crown is labiolingually more compressed.

\section{Dental wear}

Various authors mentioned the presence of dental wear in Mariliasuchus (e.g. Vasconellos and Carvalho 2005, Zaher et al. 2006, Andrade and Bertini 2008b, c, Fiorelli and Calvo 2008, Nobre et al. 2008), but none of these authors discussed the possible jaw mechanism which might have created such dental wear features. Using the results of these studies but mostly relying on personal investigation of several specimens, here I give a detailed description on the dental wear pattern of Mariliasuchus because this is one of the critical feeding related characters which leads to the understanding of the feeding mechanism of this animal. Mariliasuchus is among the few notosuchians which bear extensive wear facets on the anterior, procumbent teeth (Figure 22). Most distinct on specimens MZSP-PV 50 and MN 6756 , these teeth have strongly developed apical wear, but the other surfaces of the tooth crowns are also strongly worn. These apical wear facets are on the labial surface of both the 
upper and lower teeth so they are certainly not the result of tooth-tooth contact. In some cases, the teeth are completely eroded and the apical wear almost reaches the base of the crown so that the pulp cavity is exposed (e.g. on the left first dentary tooth of MZSP-PV 50). The plane of this apical wear facet is close to vertical relative to the long axis of the mandible, otherwise obliquely positioned relatively to the long axis of the tooth. It is important to note that these wear facets are frequently on the dentine thus their surface is generally rough and ornamented by heavy pits and scratches. Based on the wear pattern of the anterior teeth of MZSP-PV 50, pits are more frequent than scratches. Most scratches are short (maximum length $1.5 \mathrm{~mm}$ ), deep and wide, and they have no preferred orientation. Among these wide scratches, smaller, thiner ones are also present. In these strongly worn anterior teeth, most of the enamel cover appears to be missing from all sides of the tooth, thus the enamel-dentine interface cannot be studied. The margin of these apical wear facets is rounded in various degrees. Whereas the apical region of the smaller, right second lower dentary tooth is well rounded, this edge of the first dentary teeth is sharper but not as sharp as in the case of an enamel edge.

Apical wear is present on the premaxillary teeth but not as extensively as those on the dentary teeth. The left caniniform tooth of MZSP-PV 50, however, shows a developed wear facet on the mesial surface (Figure 22C). This wear facet is more steeply inclined relative to the long axis of the tooth than those on the procumbent, anterior dentary teeth because this caniniform is only slightly procumbent. The wear facet is smooth with small pits and some randomly oriented, deep and short scratches on the dentine (Figure 23(A)). The enamel-dentine interface is irregular with a higher, step-like enamel basally and labiolingually. This unambiguously indicates that this surface was abraded from ventral direction but not as the result of tooth-tooth occlusion. Beside this mesial wear facet, the caniniforms of different specimens (MN 6756, MZSP-PV 51, UFRJ 105-R) bear some apical wear sometimes with the 
dentine exposed. In addition, both caniniforms of UFRJ 106-R show extensive wear facets lingually. These almost vertical, worn surfaces are due to the shearing contact with the anterior lower teeth during occlusion (Figure 21(G, H)).

Posterior to these anterior teeth, the more stocky and serrated/cuspidate teeth possess extensive wear facets (Figure 23C-I). In general, it can be concluded that wear facets are steeply inclined ranging from $35-70^{\circ}$ relative to the horizontal plane and they are lingual on the upper teeth and labial on the lower teeth (Figure 21(C-J), 23). However in some cases, where the wear facet is so extensive, the whole apical region has been abraded and the wear facet is close to horizontal. Important to note is that the orientation and extension of wear facets show some variation among the different specimens that may be related with their different ontogenetic stages.

The small transitional tooth and the first maxillary tooth of MN 6756 possess small apical wear that is almost horizontal or slightly apicolingual. Posteriorly, the wear facets become more inclined lingually. The left second, larger tooth behind the transitional tooth in the maxilla bears more extended wear facets with an angle of approximately $45^{\circ}$ and a great mass of dentine is exposed. These oval, mesiodistally slightly longer facets are facing apicolingually (Figure 21(B)). In MZSP-PV 51, the wear facet on the second maxillary teeth are not as heavy as on the same tooth of MN 6756 but it is slightly concave and much more steeply inclined with an angle of approximately $60-70^{\circ}$. Here, the dentine is not exposed. The smooth wear surface bears two distinct types of wear pattern: first, it has apicomesialdistobasally oriented, long $(1-1.5 \mathrm{~mm})$ parallel scratches. Some of these scratches are deep and heavy. Second, it shows numerous, apicobasally oriented elongate pits or short scratches (similar to elongate arrows). These two different patterns are apparently the result of two different phases of food processing (see below). 
In UFRJ 106-R, the transitional tooth and the anterior two maxillary teeth bear two distinct wear facets. First, an oblique apical wear is present with the dentine exposed and second, a more extensive, steeply inclined lingual wear also occurs. The apical wear on the second maxillary tooth behind the transitional one is distolingually oriented, drop-shaped and possesses parallel, distolingually oriented heavy scratches on the dentine. Whereas apically the EDI is smooth on this wear facet, more basally it is more irregular. The steeply inclined and relatively smooth wear facet on the dentine of the transitional and first maxillary teeth is ornamented by a great number of pits. Here, scratches do not show a preferred orientation and whereas the apicobasally oriented scratches are usually wide, short and deep, the fewer, mesiodistal scratches are thin and shallow. The EDI on the transitional tooth is mostly exposed on the mesiodistal side of the tooth crown, it is flush and no step relationship is present.

The wear facet on the third to fifth (last) maxillary teeth behind the transitional tooth is steeply inclined $\left(45-70^{\circ}\right.$, Figure $\left.23 \mathrm{C}-\mathrm{G}\right)$. These teeth have a very thick enamel cover that is indicated by some extensive wear facets where the dentine is still not or only minimally exposed. These facets are usually on the linguodistal part of the crown generally starting from the apex. Interestingly, the last, small tooth of the left maxilla of UFRJ 106-R has an extensive wear facet on the labial side of the crown. Wear facets are dominated by apicomesial-distobasally oriented $\left(30-45^{\circ}\right.$ relative to the horizontal plane), long (maximum length 3-4 mm) parallel scratches (Figure 21(E), 23(E)). It appears that the heaviest dental wear always occurs on the fourth, penultimate maxillary tooth. On these teeth of MZSP-PV 51, MZSP-PV 50 or those of MN 6298, the lingual wear facets extend well into the apical region and also to the distal carina. This is best seen on the right penultimate maxillary tooth of MN 6298 where the distal carina is completely eroded. Here, the slightly concave wear facet is $1.2 \mathrm{~mm}$ wide labiolingually and the scratches are close to $4 \mathrm{~mm}$ in length. These 
scratches are deeply carved in the dentine and they are irregular complemented by numerous pits. The EDI cannot be clearly observed in every part of the wear facet but it is relatively flush apically and mesially whereas it is fractured and irregular basally and distally. On the other hand, due to the thick enamel cover, the extensive wear facet on the left penultimate maxillary tooth of MZSP-PV 51 is predominantly on the enamel, and the dentine (white in color) is barely exposed apically (Figure 21(E)). Here, wear pattern is dominated by fine, 1-2 mm long parallel scratches, whereas pits are significantly less numerous. There are only a few apicobasally oriented, arrow-like short scratches limited to the apical region. Except for the above mentioned labially positioned wear facet in UFRJ 106-R, the last, smallest tooth is usually devoid of any significant dental wear.

Behind the two, strongly procumbent anterior dentary teeth, the third left dentary teeth of UFRJ 106-R, still being slightly procumbent, possess extensive, slightly mesiolabially facing (ca. $20^{\circ}$ relative to the horizontal plane) circular wear facet. Posteriorly, on the fourth and fifth teeth of MN 6756, small apical wear occurs. More posteriorly on the sixth to eighth teeth, wear facets are facing more labially-mesiolabially with an angle of $40-70^{\circ}$ relative to the horizontal plane. These are nice complementary surfaces of the maxillary wear facets. Of the dentary teeth, the left sixth teeth of UFRJ 106-R show the most extensive wear pattern. Here, the labial wear facet is strongly extended apically, thus the whole apical region is eroded producing a slightly mesiolabially facing, labiolingually wide $(2.2 \mathrm{~mm})$, concave trough-like wear facet in the dentine. This surface, as usually seen on wear facets present on the dentine, is irregular with heavy pits and short deep scratches surrounded by a rough EDI.

\section{Adductor muscle reconstruction}

Due to the exceptionally preserved skulls and mandibles (e.g. MZSP-PV 50, 51, UFRJ 106-R) of Mariliasuchus, the relative extension and size of the origin and insertion surfaces and the 
orientation of the cranial adductors can be accurately reconstructed (Figure 24). One of the most significant features of the skull of Mariliasuchus is the strong reduction of the pterygoids. This unambiguously indicates a relatively less developed MPTV compared to extant crocodylians (Iordansky 1964, Figure 24(A)). The large suborbital fenestrae bordered by the mediolaterally wide and massive ectopterygoids, the mediolaterally wide palatines, and the mediolaterally wide ectopterygoid-maxilla suture below the orbit, however, suggest an advanced MPTD comparable to that of extant forms (Holliday and Witmer 2007). Close to the quadrate-quadratojugal suture, the ventral surface of the quadrates of MZSP-PV 51 bears two short, anteroposteriorly oriented, parallel bony crests that appear to correspond to the muscle scar A of Iordansky (1964). In other Mariliasuchus specimens, these crests cannot be observed. In extant forms, the A-tendon onto which some bundles of MAMP attach, arises from here. This suggests a MAMP at least as well-developed as that of extant forms (Figure 24(A)). The relatively short and approximately vertically oriented bundles of MAMES originate from the ventral surface of the quadratojugal (Holliday and Witmer 2007). In contrast to extant forms (Iordansky 1964), this surface forms a great part of the lateral temporal bar in Mariliasuchus (Figure 24(A)). On MZSP-PV 51, the ventral surface of the quadratojugal bears a posterolaterally-anteromedially oriented, shallow crest. This, along with the large laterotemporal fenestra points to an advanced MAMES. This notion is further supported by the elongate, mediolaterally wide and massive dorsal margin of the postdentary part of the mandible where this muscle attaches. The MAMEM had its insertion surface also dorsally, just medial to that of MAMES, so this muscle could also have been well-developed (Figure 24(C)).

In contrast to Notosuchus and Malawisuchus, the most characteristic osteological feature related to the cranial adductors of Mariliasuchus is the extremely enlarged supratemporal fenestrae. The self-like region of the fenestra leading to the supratemporal fossa is expanded 
even onto the posterolateral margin of the frontal and the posterior and posterolateral margins of the postorbital. This clearly suggests a highly advanced MAMEP originating from the selflike margin of this fenestra (Figure 24(B)). In extant forms, this muscle merges with the lamina pars lateralis of the mandibular adductor tendon (Busbey 1989). This tendon attaches partly to the dorsolateral part of the cartilago transiliens (Busbey 1989) and partly to the anterodorsal edge of the surangular (Holliday and Witmer 2007). This anterodorsal edge of the surangular and the elongate and wide posterior process of the dentary are massive and they certainly served as attachment areas for these tendinous structures. Ventral to the parietal the medial surface of the supratemporal fossa is formed by the laterosphenoid (Zaher et al. 2006, pers. obs.). Due to the expanded nature of the supratemporal fossa a relatively larger origin surface of MPSS can be reconstructed compared to modern forms, although no particular muscle scar or ridge are present on this surface. This suggests that MPSS was at least comparable or more developed in size to that of extant forms. This is in accordance with the well developed MAMEP suggesting powerful posterodorsal-anteroventrally oriented muscles originating from supratemporal region.

To sum up, the development of the cranial adductors in Mariliasuchus was considerably different from that in the earlier discussed notosuchians such as Malawisuchus and Notosuchus. Mariliasuchus had an extremely developed and relatively more backward originating, posterodorsal-anteroventrally oriented MAMEP. MPSS could be also well developed in Mariliasuchus. MAMES and MAMEM were also well developed. MAMP could have been comparable to those of extant forms, but the MPTV may have been strongly reduced. 
Based on dental characters, dental wear and jaw joint morphology, several authors (e.g. Andrade 2005, Zaher et al. 2006, Andrade and Bertini 2008b, c, Nobre et al. 2008) suggested the anteroposterior shifting of the mandibles during jaw closure in Mariliasuchus. Indeed, the occurrence of an anteroposterior component during the closing phase of the jaw movement can be demonstrated, which, however, was strongly different from that seen in Malawisuchus or Chimaerasuchus. Whereas in the latter two taxa, proal movement with shearing carinae can be reconstructed, the jaw movement of Mariliasuchus was unambiguously palinal. In Mariliasuchus, the anteroposteriorly elongate mandibular glenoid surface without any buttress posterior to it (Figure $25(\mathrm{~F}, \mathrm{G})$ ) clearly suggests that the mandibles could shift in an anteroposterior direction. The symphysis is strongly ossified thus both mandibles moved synchronously and there was no axial rotation of the hemi-mandibles. The fused symphysis, the elevated posterior part of the surangular lateral to the glenoid surface, and the short distance between the lateral side of the pterygoid-ectopterygoid complex and the mandibles in closed position prevent the mandibles to translate significantly lateromedially or rotate in the horizontal plane. These features along with the distinct and relatively steeply inclined wear facets on the posterior teeth indicate a precise dental occlusion and a sophisticated mandibular movement in Mariliasuchus.

Contrary to the dental wear features of Notosuchus and Malawisuchus which rather occur on the single, functional carina (distally on the upper and mesially on the lower teeth), wear facets in Mariliasuchus are predominantly lingual to the distal carina of the upper and labial to the mesial carina of the lower teeth. The apicomesial-distobasally oriented, long, parallel scratches of the wear facets and the flush EDI of the upper teeth unambiguously indicate that during jaw closure the mandibles moved upwards and backwards, i.e. a retractive powerstroke was involved in the oral food processing of Mariliasuchus. The apicodistal-mesiobasally oriented, parallel scratches and the step relationship of the EDI mesiobasally on the lower 
teeth further support this hypothesis. However, the orientation of these parallel scratches $(30$ $40^{\circ}$ relative to the horizontal plane) and the extremely low number of horizontal scratches (some short horizontal scraches occur on the occluding side of the anterior teeth and on the extensive, apical wear facet of some strongly worn posterior teeth) indicate both anteroposterior and vertical components of the jaw closure in Mariliasuchus. The longest apicomesial-distobasally oriented scratches are 3-4 $\mathrm{mm}$ long that refer to a 2-3 $\mathrm{mm}$ long horizontal component. Palinal jaw movement during the powerstroke occured in a variety of groups of synapsids. Among these, multituberculates are a diverse group with clear retractive powerstroke and here the powerstroke was directed completely posteriorly (Gambaryan and Kielan-Jaworowska 1995). On the other hand, backward chewing occurred also in the traversodontid cynodonts, in which the powerstroke was directed posterodorsally, rather than entirely posteriorly (Crompton 1972). This means that in some lineages of mammals (and possibly in other non-mammalian synapsids), the direction of powerstroke was not simply proal (as in rodents) or palinal but it included some dorsal or lateromedial components. The retractive powerstroke, i.e. the palinal jaw movement of Mariliasuchus is further supported by its reconstructed cranial adductor musculature. Whereas in Malawisuchus (Clark et al. 1989) and perhaps in Notosuchus, the anteriorly originated pterygoid muscles have been suggested to protract the mandibles during jaw closure, in Mariliasuchus these muscles were relatively weakly developed. However, MAMEP originating from the supratemporal fenestra and inserting anteriorly was extremely developed (in contrast to Malawisuchus and Notosuchus). The orientation of this muscle must have been posterodorsal-anteroventral (Figure 24(D)), hence this muscle was certainly one of the main motors involved in the backward pulling of the mandibles in the closing phase. In addition, the MAMES and MAMEM origintaing ventrally form the quadrate-quadratojugal complex and inserting anterodorsally on the surangular must have had also some anteroposterior component thus 
they might have also been involved in the rectractive powerstroke. A convergent reduction of the pterygoideus muscles and the enlarged mass and more anteroposterior orientation of the external adductors have also been described in various non-mammalian synapsids (e.g. anomodonts). They too applied an effective retraction of the mandible during powerstroke that was enhanced by far anterior insertion of the external adductors attaching on the backward extension of the temporal fenestra (King 1996). On the other hand, pterygoideus muscles became reduced. Both in anomodonts and in Mariliasuchus (and also in sphagesaurids, see later), these changes provided not only a greater bit force when teeth were occluded but also a significant anteroposterior component in jaw movement to provide more effective backward pulling of the mandibles during the powerstroke and occlusion. Because some weak deformation of the Mariliasuchus specimens is always present, it is difficult to assess whether dental occlusion was unilateral with alternating working and balancing sides in one chewing cycle as in various mammals (Hiiemae 2000) or it was simply bilateral as it occurs, for example, in extant lepidosaurs. Unilateral dental occlusion is known for example in sphagesaurid crocodyliforms (Pol 2003, Marinho and Carvalho 2009) where the difference between the distances of the left and right lower and upper teeth is so large that dental occlusion can not be explained in any other way. Measuring the two occluding pairs of teeth in the case of the well-presereved MZSP-PV 51 specimen of Mariliasuchus, the distance between the apexes of the third, left and right maxillary teeth is $2.8 \mathrm{~cm}$ and that of the seventh dentary teeth is $2.4 \mathrm{~cm}$, so the difference is only $4 \mathrm{~mm}$. This difference is $6 \mathrm{~mm}$ in UFRJ 106R. This indicates that in a symmetrical arrangement, the distance between the apexes of the ipsolateral upper and lower teeth was $2-3 \mathrm{~mm}$. This distance seems to be too much (crown width of the posterior teeth is approximately 3-4 $\mathrm{mm}$ ) to produce the detected wear facets simultaneously on both sides (i.e. bilateral occlusion). A simple, bilateral occlusion may have existed, but a unilateral dental occlusion is a more feasible explanation which, in contrast to 
sphagesaurids (see below), required only minimal (1-2 mm) translational or rotational movement of the fused mandibles.

Palinal mandibular movement occurs in some extant turtles (most developed in gopher tortoises, Bramble 1974) and has been reconstructed, for example, in dicynodont synapsids (King 1996, Rybczynski and Reisz 2001) and multituberculate mammals (Gingerich 1977, Krause 1982, Gambaryan and Kielan-Jaworowska 1995, Butler 2000). In herbivorous dicynodonts and in multituberculates, oral food porcessing appears to have been more complex and effective than in Mariliasuchus. In the Late Permian dicynodont Suminia, the labiolingually flattened, coarsely serrated teeth are closely packed and the scratches on the wear facets indicate that ,each of the occluding lower teeth would have contacted two or three upper teeth” (Rybczynski and Reisz 2001:686). In Mariliasuchus, the widely packed teeth, relatively small wear facets, and the orientation of the scratches all indicate that one occluding lower tooth contacted only one upper tooth, so the shearing mechanism was not as effective as in the herbivorous Suminia. Krause (1982) pointed out two cycles of mastication (the slicingcrushing cycle performed mainly by the premolars and the grinding cycle by the molars) in the multituberculate Ptilodus that is strongly related to its highly differentiated dentition (i.e. large, crest-like premolars and flat cuspidate molars). Besides tooth morphology, this hypothesis was based on two different generations of wear pattern (i.e. formed in different phases of mastication) among which the horizontal scratches on both the premolars and molars indicate a distinguished phase of mastication with significant retraction of the mandibles. In Mariliasuchus, horizontal scratches are minimal (mostly on the anterior teeth), thus a clear horizontal component of mandibular movement cannot be confirmed. Assessing the information described above, the following phases of oral food processing can be reconstructed (Figure 25(A-E)): 
1) At the beginning of the preparatory stroke, the mandibles are in a closed, retracted position. During opening and closing of the mouth, the mandibles shifted slightly forward along the elongate glenoid surface-quadrate articular surface as seen in gopher turtles (Bramble 1974). In this phase, MDM attaching on the retroarticular process opens the jaws. Protraction of the mandibles was probably initiated by the pterygoideus muscles (perhaps by MPTV which has slightly larger anteroposterior component than that of MPTD when mandibles are open) as it was also reconstructed in multituberculates (Gambaryan and Kielan-Jaworowska 1995, Figure 25(C)). Preparatory stroke continues with the closing of the mouth that was motorized by the pterygoid muscles, and the posterior (MAMP) and external adductors (MAME, MAMEM, MAMEP). In various rodents and taeniolabidoid multituberculates, a significant action of the incisors (i.e. biting) is repeated several times (Weijs 1975, Gorniak 1977, Gambaryan and Kielan-Jaworowska 1995) that resulted in the appearance of extensive wear facets on the incisors. In Mariliasuchus, the food was captured by the procumbent premaxillary and anterior dentary teeth which are supposed to have had a grasping and holding rather than a gnawing function. This implies that the phase involving the action of incisiviforms was probably shorter than in the referred mammals. Backward movement of the mandibles was probably absent or not siginificant in this phase.

2) When the food was in the oral cavity, powerstroke was set on. Differentiation of the postcaniniform dentition of Mariliasuchus indicates two different phases in the oral food processing similarly to mutitiberculates (Krause 1982) or the eusuchian crocodyliform Iharkutosuchus (Ösi and Weishampel 2009). First, a crushing-puncturing phase occured when the anteriorly positoned, more pointed, conical teeth (the transitional and first maxillary teeth and the $3^{\text {rd }}$ to $5^{\text {th }}$ dentary teeth with small, mostly apical wear) crushed the food item into smaller pieces (Figure 25(D)). Thereafter the larger, more robust posterior teeth came into action with precise dental occlusion and efficient food processing. The irregular and cuspidate 
surface of these teeth was a perfect tool for grinding the food. Retraction of the mandibles was most expressed in this phase. The obliquely oriented scratches on the wear facets indicate that this retraction was not a separate phase as in gopher turtles and multituberculates (i.e. closure of the mandibles and then retraction) but it was simply involved at the end of the closing phase of the mandibles (Figure 25(D-A)). Similarly to gopher turtles (Bramble 1974), retraction of the mandibles was motorized by the highly developed external adductors, namely the MAMEP and MAMEM. In this phase, the posterior teeth came into a shearing contact during occlusion, and during retractive power stroke, the mandibular teeth moved posterodorsally. If unilateral occlusion was indeed present in Mariliasuchus, then the similar wear pattern observed on the teeth of both sides indicates frequent side switching of food similarly to Sphenodon and Malawisuchus.

3) When food was completely triturated, the power stroke completed with palinal movement stopped, and food was transported to the posterior part of the oral cavity and swallowed.

Sphagesauridae Kuhn, 1968

Armadillosuchus Marinho and Carvalho, 2009

Armadillosuchus arrudai Marinho and Carvalho, 2009

(Figure 26-28)

Holotype-UFRJ DG 303-R, partially complete skull, isolated but associated teeth and postcranial remains.

Referred specimens-MPMA-64-0001-04, rostrum and corresponding part of the fused mandibles. In addition, some unpublished specimens are referred to this species (Thiago Marinho, pers. comm., 2010). 
Type Locality_-General Salgado County, São Paulo State, Brazil (Marinho and Carvalho 2009).

Type Horizon-Upper Cretaceous, Adamantina Formation, Bauru Group. As discussed in Mariliasuchus, the age and stratigraphical context of the fossiliferous Adamantina Formation (including its basal member, the Araçatuba Formation, Fernandes et al. 2003) is not clarified yet. So, up to the present, it is unclear how Mariliasuchus, Adamantinasuchus, Armadillosuchus, and other sphagesaurids related to each other in time.

\section{General cranial and mandibular morphology}

The Late Cretaceous Armadillosuchus is the largest heterodont notosuchian with a skull length of $27.7 \mathrm{~cm}$ (Figure 26(A-C)). This is 3-4 times larger than the skull of Malawisuchus and Candidonon, 2.5-3 times larger than that of Mariliasuchus and almost 1.5 times larger than that of Notosuchus (RN 1037) and Sphagesaurus (RCL-100). The rostrum is almost three times narrower than the width of the posterior skull. According to Busbey (1995), Armadillosuchus is platyrostral because the rostrum is lateromedially much wider than high. The relatively flat rostrum is rounded anteriorly and its lateral margins are slightly divergent posteriorly. Whereas the dorsal surface of the rostrum is sculptured by grooves and pits as generally seen in crocodyliforms, the surface of its lateral margins is smooth as in Sphagesaurus (Pol 2003, Andrade and Bertini 2008a) but strongly irregular. In the level of the prefrontal-lacrimal suture (Marinho and Carvalho 2009), the skull becomes abruptly wide with massive lateroventral margin of the orbit and lower temporal arcade lateral to the laterotemporal fenestra. The latter, triangular opening is the largest fenestra of the skull, even larger than the triangular supratemporal fenestra (Figure 26(C)). Similarly to Mariliasuchus, the supratemporal fossa and supratemporal fenestra are huge with the margin of the fenestrae comparable with the size of the orbit. The flat skull roof has strongly divergent lateral margins 
that posteriorly end in massive squamosal-suprasquamosal complexes (Marinho and Carvalho 2009). Quadrates of Armadillosuchus are short and ventrally oriented. The smaller, rounded medial quadrate condyle is separated from the lateromedially wider lateral condyle by a shallow groove. The occipital condyle is not preserved, thus it is not clear whether it had a posteroventral orientation as ususally seen in Notosuchians. The secondary bony palate is poorly preserved. Its anterior end is covered with matrix on the holotype specimen but it can be observed on MPMA-64-0001-04. It is massive and at least at the level of the $3^{\text {th }}-4^{\text {th }}$ maxillary teeth, it is formed by the premaxilla and maxillae. The palatines, pterygoids and ectopterygoids are not preserved, thus the extension of the suborbital fenestrae is unknown. However, reconstruction of mandibular movement in Armadillosuchus indicates side-to-side (lateromedial) movement of the jaws (see below). This implies lateromedially slender pterygoid flanges that allow the mandible to shift lateromedially during jaw closure. Of the mandibles, only the anterior, symphyseal part is preserved in MPMA-64-0001-04 (Figure 26(D-H)). At the posterior end of the strongly fused, elongate symphysis, the anterior ends of the splenials are present in ventral view, and at this level, the hemi-mandibles become slightly divergent. The postdentary part of the mandibles including the jaw joint is unknown but in lateral view the dorsoventrally strongly widened dentaries suggest a massive postdentary part to accomodate a huge mass of cranial adductors.

\section{Dentition and possible extant analogues}

Corresponding to the relatively large sized skull, the heterodont dentition of Armadillosuchus is composed of large, conical, carinated teeth bearing complex ornamentation on their surface (Figure 26-28). Tooth crowns are relatively widely spaced and they do not overlap each other. In the holotype skull, the preserved alveoli and fragmentary teeth are covered with matrix and only two isolated but associated teeth (Marinho and Carvalho 2009: fig. 4) can be 
studied from the type material. Dentition of MPMA-64-0001-04 is more complete both in the upper and lower jaws. The upper tooth row behind the third maxillary tooth becomes divergent. The characteristic longitudinal enamel ridges as seen in other sphagesaurids (Pol 2003, Andrade and Bertini 2008a) are present on all teeth of Armadillosuchus. In addition, the unworn enamel surface of all preserved teeth has a coarse, irregular texture.

Marinho and Carvalho (2009) described two premaxillary teeth. However, based on the paratype MPMA-64-0001-04, it appears that the premaxilla-maxilla suture between the alveoli occur directly behind the third alveoli suggesting three premaxillary teeth similarly to Mariliasuchus. Hopefully additional, still unpublished material (Thiago Marinho, pers. com. 2010) will help in clarifying this problem. The first premaxillary tooth is not preserved in any of the specimens but the right, first premaxillary alveolus of the holotype indicates that this tooth was relatively small and slightly procumbent. The second premaxillary tooth is a hypertrophied (apicobasal length ca. $3.5 \mathrm{~cm}$ ), distally curved caniniform with circular crosssection which, besides the approximately 20 marked, longitudinal enamel ridges, bears neither distal nor mesial carinae (Figure 26(D, E), 27, (F, G)). The third tooth in the upper jaw (probably the third premaxillary tooth) has an apicobasal length of approximately $3.5 \mathrm{~cm}$ and it is still conical with distally curved, non-carinated crown and circular cross-section. Posteriorly to these distally curved teeth, the exact number of maxillary teeth is unknown. They have a drop-shaped cross-section as in other sphagesauirds and they bear a stark, lingually and slightly distally oriented carina (Figure 26(D, E, H), 26(A, B)). In MPMA-640001-04, three teeth are preserved in the right and one in the left maxilla, all of them with worn carinae. However, the associated teeth of the holotype (Marinho and Carvalho 2009: fig. 4) show the details of the massive, blade-like and cuspidate/tuberculated carina that starts from the apex and reaches the base of the crown. The labiolingual depth of this compressed carina is $3-4 \mathrm{~mm}$ in the middle of the crown. The size of the tubercles on the carina is 
variable ranging from 1 to 2.5 millimeters. The three teeth in the maxilla are roughly equal in size and similar in morphology, and they are slightly distally, mediodistally curved. Only the five anterior dentary teeth are preserved in Armadillosuchus. They are from the relatively narrow, symphyseal part of the mandibles where the left and right teeth are very close to each other (Figure 26(F, G), 27(A-C)). In the case of the fourth teeth, the distance between them equals to the width of one of these teeth. The first tooth is a huge, most probably conical and non-carinated, strongly procumbent tooth. Only the roots of both first dentary teeth are preserved in MPMA-64-0001-04. The second and third dentary teeth are much smaller (the right, second tooth is $1.3 \mathrm{~cm}$ ), conical, non-carinated and has a circular cross-section. Both of them are still strongly procumbent but have a distal curvature. The fourth tooth is the largest, it is slightly lingually curved and weakly procumbent, and has a mesially facing, slightly convex, tuberculate carina. Due to the orientation of the tooth crown, this mesial carina has a vertical position (Figure 28(A)). In the massive roots of the fourth teeth, replacement teeth with similar enamel ornamentation can be observed. The fifth tooth is preserved only in the right dentary. It is slightly smaller than the fourth tooth and has a mesiolabially oriented, tuberculate carina.

Dentition of Armadillosuchus (and other sphagesaurids) is generally similar to that of Notosuchus in having subtriangular, pointed crowns with roughly drop-shaped cross-section and single carina oriented obliquely relative to the saggital plane. However, the exact orientation of the carinae are different, because whereas in Notosuchus upper tooth carinae are linguodistally, distally oriented, upper tooth carinae in Armadillosuchus are rather lingually, slightly linguodistally oriented. Due to their large size and unique morphology, teeth of Armadillosuchus are hardly comparable with those of extant reptiles. 
Due to the large size of the teeth of Armadillosuchus, the majority of the wear pattern is macroscopic and some details (e.g. scratch orientation) can be easily observed (Figure 27, 28). Generally, it can be concluded that teeth of Armadillosuchus bear two sets of wear: 1) apical wear that mostly occur on the lower teeth and anterior upper teeth (Figure 28(D)) and 2) steeply inclined, lingual and labial wear on the upper and lower teeth, respectively (Figure 27). The anterior enlarged caniniforms in the premaxilla of MPMA-64-0001-04 show both of these wear types. The apical region is finely eroded that resulted in a smooth surface that also extends onto the apical part of enamel ridges. Besides apical wear, these caniniforms possess almost vertically positioned, flat, lingual wear facets that extend from the apex to the base of the crown (Figure 27(F-H)). On both caniniforms, the enamel is eroded from large areas, and on the right one close to the apex, the wear penetrate deep into the dentine. These lingual wear facets show two different sets of scratches. One of them is also related to the apical wear. They are apicobasally or slightly apicomesial-distobasally oriented, have an inclination of approximately $10-15^{\circ}$ relative to the vertical plane, and are roughly parallel with the axis of the crown. These scratches are usually short $(100-200 \mu \mathrm{m})$, and on the eroded dentine surface they are frequently arrow-like (deeper and wider basally). The second set of scratches is apicomesial-distobasally oriented with an inclination of about $10-20^{\circ}$ relative to the horizontal plane. They are long and parallel scratches. Due to the two different directions of dental wear, the EDI on the caniniform teeth is not as marked as in the case of teeth working in a simple (e.g. orthal) jaw movement. Nevertheless, a weak step relationship of EDI on the right tooth of MPMA-64-0001-04 can be observed on the distal side of the crown. Posterior to the huge caniniforms, the probably third premaxillary teeth bear weak apical wear but no lingual wear facets can be observed.

In MPMA-64-0001-04, except for the first left maxillary tooth, all maxillary teeth possess flat and distinct wear facets lingually on the tuberculate carina penetrating deep into the dentine. 
These wear facets are facing lingually, ventrolingually (approximately $20^{\circ}$ relative to the vertical plane) and represent the complementary surfaces of the wear facets on the dentary teeth. Wear facets of the right three preserved maxillary teeth are approximately in the same plane. They are flat and rectangular in shape. Wear pattern is dominanted by parallel scratches that are oriented mesiodistally or apicomesial-distobasally inclined approximately $15-30^{\circ}$ relative to the horizontal plane (Figure 27(A-E)) but some apicobasally oriented scratches also occur.

Dental wear on the preserved mandibular teeth (MPMA-64-0001-04) are more extensive than those of the maxillary teeth because the teeth bear both apical and labial wear that are frequently connected with each other. Although they are not preserved, the massive, procumbent, first dentary teeth most probably were eroded at least on their labial surface as it is indicated by the wear pattern of the hypertrophied caniniform of the premaxilla. Of the second dentary teeth, the tip of the left one is broken and the right one bears some wear apically but the enamel is not eroded yet. The left third, still procumbent dentary tooth shows some weak labial wear but interestingly the apical wear that extends lingually is much more developed. This wear facet has a mesiolingual orientation with an angle of approximately 45 $50^{\circ}$ relative to the vertical plane. In contrast to the left one, the right third tooth bears more extensive labially facing wear besides the apical and weak lingually facing wear facets. In the third dentary teeth, the angle of the mesially facing, flat apical wear is approximately $45^{\circ}$ relative to the horizontal plane.

The fourth, largest dentary teeth possess the most extensive dental wear in the dentition of Armadillosuchus. These teeth show the most complex wear pattern among crocodyliforms being composed of wear facets in four different directions. Almost the half of the crown of the left fourth tooth became eroded. The greatest part of wear occurs labially. This extensive, flat wear facet is steeply inclined by approximately $20^{\circ}$ relative to the vertical plane and 
represents the complementary surface of wear facets on the left maxillary teeth. Most of the wear facet is on the dentine and wear pattern is dominated by obliquely oriented (approximately $35-40^{\circ}$ relative to the horizontal plane), long and parallel scratches. A distinct, mesiodistally elongate apical wear facet is present that is parallel with the orientation of the labial scratches (Figure 28(B-D)). Here, the dentine surface is not as smooth as on the labial facet but ornamented by heavy pits and the mesiodistally positioned enamel coat is also irregular. Similarly to the third dentary teeth, the fourth teeth also bear wear facets lingually. This is most developed on the left tooth being approximately one fourth of the labial facet. It is a distinct, lingually and slightly mesially facing, flat surface in the dentine. Because most of the tooth became eroded, the mesially positioned and vertically oriented tuberculate carina is missing. However, at the base of the crown, approximately one third of the carina is still present but the tuberculate enamel coat was abraded. This apicobasally elongate wear facet is facing mesiolabially. Compared to the left one, the right fourth tooth bears only weakly developed wear facets (Figure 28(A)) which are mostly on the enamel. Their position (most developed labially, then apically and lingually) and orientation are identical with those on the left tooth. The worn enamel surfaces show elongate $(2-3 \mathrm{~mm})$ scratches with an orientation similar to those on the left side. The mesial carina of this tooth is more complete than that of the left tooth bearing some wear only apically. The EDI is smooth on most parts of the fourth teeth and a typical step relationship cannot be observed. Only the right fifth dentary tooth is preserved. It seems that it is just erupted, thus it is almost complete with weak labial and apical wear facets on the enamel.

\section{Adductor muscle reconstruction}

The pterygoids and the suborbital region of the skull and the postdentary part of the mandible of Armadillosuchus are not preserved thus the reconstruction of the pterygoid muscles is not 
possible. The surrounding bones of the temporal fenestrae, however, are well preserved and allow identification of the muscle origins on their surfaces. Similarly to Mariliasuchus, the triangular supratemporal fenestrae and the shelfs leading to the supratemporal fossa in Armadillosuchus are huge relative to the skull roof. The ornamented dorsal part of the marginal bones bordering the fenestrae (squamosal, postorbital, parietal) are only narrow surfaces. Besides the bones surrounding the supratemporal fenestrae, those bordering the supratemporal fossa (e.g. laterosphenoid) are also extended. This unambiguously indicates extremely developed MAMEP (Figure 26(A)) and, similarly to Mariliasuchus, suggests developed MPSS in this crocodyliform. The ventral surface of the quadrate bears two distinct areas of muscle or tendon attachments. First, in the anterior part, a shallow ridge (approximately $2-3 \mathrm{~cm}$ long) is present and especially pronounced on the left quadrate. This area along with the proportionally very robust quadrate suggests an advanced MAMP that could have been comparable with that of modern forms. Second, the laterotemporal fenestra is bordered posteriorly by the quadrate because the quadratojugal does not extend so much anteriorly. Along this anterior margin of the quadrate and roughly parallel with the above described shallow ridge, a prominent crest can be observed. In modern forms, MAMEM originates from this area (Busbey 1989, Holliday and Witmer 2007), thus it can be suggested that bundles of this highly advanced muscle attached here in Armadillosuchus too. In addition, the quadratojugal is unusual compared to most crocodyliforms because it lacks the elongate anterior process along the quadrate but it forms the massive and wide posterior corner of the laterotemporal fenestra. This surface shows an extended and strongly posteriorly positioned origin surface for a higly advanced MAMES. To sum up, the preserved origin surfaces of cranial adductors indicate highly advanced parts of the external mandibular adductors (MAME) in Armadillosuchus. 


\section{Jaw mechanism and possible phases of chewing}

Although the postdentary part of the mandible of Armadillosuchus including the glenoid surface of the articular is not preserved, the dentition, individual tooth morphology, preserved wear pattern and the reconstructed jaw adductors are perfect morphological correlates of dental occlusion thereby permitting the reconstruction of oral food processing and possible phases of jaw mechanism. Marinho and Carvalho (2009:40) assessed that the „occluding movement included a large anteroposterior component” and they suggested a ,propalinal fore-aft jaw movement and an alternate unilateral jaw occlusion pattern" in Armadillosuchus. Indeed, wear pattern clearly indicates a significant anteroposterior component of jaw movement when the upper and lower teeth occluded. However, the details of wear facets and dental morpology indicate that jaw closure was particularly complex relative to other crocodyliforms (except for Sphagesaurus and perhaps Iharkutosuchus) and that jaw movement included orthal (simply vertical), palinal (retractive) and transverse components but no proal (protractive) movement during powerstroke.

In contrast to Mariliasuchus, wear facets in Armadillosuchus frequently bear short, apicobasally oriented scratches (e.g. lingually on the caniniform teeth and labially on the dentary teeth), although their number is far below that of the mesiodistally or apicomesiallydistobasally oriented long scratches. Nevertheless, these apicobasal scratches definitely refer to simple orthal jaw closure and show that the upper and lower teeth, although not frequently, occluded with each other. Dental occlusion was more significant in the retractive powerstoke that is unambiguously indicated by the strongly worn tooth crowns and the great number of long scratches. The mesiodistal or apicomesial-distobasal orientation of these scratches (most of them detected on the dentine) denotes that the massively fused mandibles moved backwards and slightly upwards. These scratches unambiguously show that dental occlusion (i.e. shearing contact of the teeth) was precise. Food processing was made by well-controlled 
jaw closure in spite of that the jaw mechanism of Armadillosuchus was highly influenced by an alternating jaw closure (see below). Another important difference compared to Mariliasuchus is that wear facets on the posterior maxillary teeth are mostly on the tuberculate carina, they are apicobasally extended elongate surfaces, and the orientation of scratches suggests that one mandibular tooth could easily occlude with two maxillary teeth via retraction of the mandibles. In this retraction phase, the lower jaw could have slided $1.5 \mathrm{~cm}$ posteriorly. Even if this hypothesis cannot be supported by other evidence (e.g. anteroposteriorly extremely elongate glenoid surface of the articular), the longest scratches preserved on the fourth mandibular teeth indicate a significant, 4-6 $\mathrm{mm}$ posterior shifting of the mandibles. Oral food processing was highly advanced in Armadillosuchus. Due to the active dental occlusion, abrasion of the teeth could have been a fast process (relative to tooth size and to other crocodyliforms) which notion, besides the extensive wear facets, is further supported by the preserved replacement teeth.

Similarly to Sphagesaurus (Pol 2003), an alternating jaw movement was noted in Armadillosuchus (Marinho and Carvalho 2009). Reconstruction of the mandibles and the preserved rostrum of MPMA-64-0001-04 in a closed position clearly supports this hypothesis. When the upper and lower teeth are in occlusion on the right side, the contralateral teeth of the lower jaw on the left side are not close enough to the upper teeth to simultaneously ensure tooth-tooth contact (Figure 26(H)). This shows that some kind of side-to-side movement of the mandibles was present and that it was a significant element of the powerstroke. The extended mandibular symphysis is strongly ossified, thus the rotation of the hemi-mandibles along their axis, as occurs in various lineages of mammals (Crompton and Hiiemae 1970, Crompton 1995, but see Lieberman and Crompton 2000), was not possible. The quadrate condyles of Armadillosuchus are separated by a shallow groove indicating a longitudinal ridge on the glenoid surface of the articular; consequently, the precisely articulated condyles 
suggest a lower degree of transverse mobility of the lower jaws. Instead of a pure lateromedial translation of the quadrate condyles in the glenoid surface, a rotation about a pivot point close to the quadrate condyle as in mammals (Mills 1967) or perhaps the combination of these movements must have been carried out during the transverse jaw movement. Dental wear facets, however, neither have labiolingual orientation, nor bear labiolingually oriented scratches. This indicates that the lateromedial movement of the lower jaw ensured the alternation of dental occlusion between the left and right sides rather than provided effective occlusion and food processing by transverse mandibular movement which is clearly the case e.g. in the basal eusuchian Iharkutosuchus (Ösi and Weishampel 2009).

Concerning the complex jaw mechanism of Armadillosuchus, the reconstructed jaw adductors well support this hypothesis. Similarly to Mariliasuchus, the palinal movement as the most significant element of jaw mechanism in the oral food processing was motorized by the strongly developed external adductors (MAMEP, MAMES, MAMEM). Orientation of these muscles had to have a great anteroposterior component to fulfill the backward shifting of the mandibles. Similarly to Iharkutosuchus, the side-to-side movement of the mandibles must have been implemented mainly by the pterygoid muscles because they have the greatest lateromedial component. This, however, does not mean that other muscles possessing smaller but significant lateromedial component in their orientation (e.g. MAMEP) could not have acted during the transverse jaw movement.

Based on this information, the following phases of chewing can be reconstructed in Armadillosuchus:

1) In the beginning of the preparatory stroke, mandibles are in a retracted position. This phase starts with the opening of the mouth by contraction of MDM. This movement and the following closing of the jaws are probably associated with the protraction of the mandibles mototized by the pterygoid muscles which have great anteroposterior component. This is 
followed by the first occlusal contact between the premaxillary and anterior dentary teeth or between these teeth and the food takes place. The mostly procumbent anterior teeth grasp and hold the food item, and probably with the help of rapid neck movements and the tongue food is taken it into the mouth. Contraction of the adductor muscles is probably repeated couple of times and this produces the mainly apicobasally oriented, short scratches on the wear facets of the anterior teeth. The direction of mandibular movement during the preparatory stroke is mostly orthal and only minimal transverse movement is supposed in this phase.

2) In the powerstroke, chewing is charaterized by palinal jaw movement and continues with a more intensive food processing performed by the posterior teeth. Contraction of MAMP, MPT and especially the external adductors motorize every cycle of jaw closure when a significant retraction of the mandibles occurs. Dental occlusion (most probably one lower tooth occluded only with one upper tooth) produces rapid wear with labial and lingual wear facets and apicomesial-distobasally oriented, parallel scratches. In this phase, the food was completely triturated by the shearing contact of the upper and lower carinate teeth making it ready to swallow. Alternation of tooth-tooth contact between the left and right sides is an important factor in this phase indicating unambiguously that the jaw apparatus of Armadillosuchus was functionally divided into a working and a balancing side as it is in Sphagesaurus (Pol 2003) and in most herbivorous mammals today (Hiiemae 2000). This condition strongly differs from modern crocodylians with no differential bilateral muscular activity (Busbey 1989) and denotes that the action of adductor musculature at least in sphagesaurids and Iharkutosuchus (and perhaps in other hylaeochampsid eusuchians) was more complex during jaw closure.

Notosuchia Gasparini, 1971

Sphagesaurus Price, 1950 and 'Caipirasuchus paulistanus' Iori and Carvalho, 2011 
(Figure 29-33)

Remarks - Sphagesaurus was described on the basis of two isolated, but well preserved teeth (Price 1950), so the additional specimens have been assigned to this genus based on their tooth morphology (Bertini et al. 1993, Kellner et al. 1995, Pol 2003). However, the morphology of the posterior teeth of Armadillosuchus is almost identical with the type teeth of Sphagesaurus. In addition, the teeth described by Price (1950) are closer in size to the teeth of Armadillosuchus than to the smaller teeth of the Sphagesaurus specimens. So, it cannot be excluded that the original teeth discovered by Friedrich Huene (Price 1950) are actually from another sphagesaurid, perhaps Armadillosuchus. However, if the latter is the case, then the Armadilloschus material should be referred to as Sphagesaurus huenei and all the additional Sphagesaurus specimens should get a new name.

Sphagesaurus includes two species, S. huenei and S. montealtensis. The data of systematic paleontology of both species are listed here separately. In the functional morphological part, however, description refers to both species because they apparently do not exhibit any anatomical deviation that might be related to different jaw mechanism and food processing.

Sphagesaurus huenei Price, 1950

Holotype-DGM 332-R, isolated maxillary tooth.

Referred specimens_-Fragmentary skull and mandibular fragment (RCL-100, Pol 2003), rostrum fragment (DGM 1411-R) and an isolated tooth (DGM 333-R, URC-R 015) (Price 1950, Bertini et al. 1993; Kellner et al. 1995).

Type Locality—Railway cut between the cities of Presidente Bernardes and Santo Anastacio, São Paulo State, Brazil (Price 1950). 
Type Horizon-Upper Cretaceous, Adamantina Formation, Bauru Group (Bertini et al. 1993).

Sphagesaurus montealtensis Andrade and Bertini, 2008a

Holotype-MPMA 15-001/90, the only known specimen including the skull and naturally articulated, fragmentary mandible (Figure 29).

Type Locality—Bairo Cachoeira, eight km northwest from the city Monte Alto, São Paulo State, Brazil (Andrade and Bertini 2008a).

Type Horizon-Upper Cretaceous (Campanian to Maastrichtian), Adamantina Formation, Bauru Group (Andrade and Bertini 2008a).

Caipirasuchus Iori and Carvalho, 2011

Holotype-MPMA 67-0001/00, skull and mandible (Figure 30).

Type Locality — Bauru basin, six kilometers from Monte Alto, in Homem de Mello, the rural area of Monte Alto County, São Paulo State, southeastern Brazil (Iori and Carvalho 2011). Type Horizon—Upper Cretaceous, Bauru Group, Adamantina Formation. (As noted earlier, the age of the Adamantina Formation is ambiguous. Whereas Andrade and Bertini, [2008a] suggested a Campanian to Maastrichtian, Iori and Carvalho [2011] used a Turonian to Santonian age).

Remarks - In my opinion, Caipirasuchus is a junior synonym of Sphagesaurus. During my personal study of the holotype specimen, I could not find any significant character that unambiguously distinguishes this specimen from the holotype of Sphagesaurus montealtensis. In addition, this specimen is almost identical with $S$. montealtensis from a functional morphological point of view too, so I used this specimen also in the following analysis and referred to it as 'Caipirasuchus' (MPMA 67-0001/00) or simply used the inventory number. 


\section{General cranial and mandibular morphology}

Sphagesaurus has a relatively large, strongly oreinirostral skull with a total length ranging from $17 \mathrm{~cm}$ (MPMA 15-001/90) to $22 \mathrm{~cm}$ (RCL-100). Behind the short and massive rostrum, the skull is still narrow (Figure 29(A), 30(A)) in contrast to the posteriorly strongly widening skulls of Mariliasuchus or Armadillosuchus. The confluent external nares are facing anteriorly. Orbits are large, anteroposteriorly slightly elongate and face laterally, anterolaterally. The triangular laterotemporal fenestrae are slightly smaller than the orbits and they are bordered lateroventrally by a rod-like lower temporal arcade. Similarly to Mariliasuchus and Armadillosuchus, the supratemporal fossa is wide and opens into the skull roof by an extremely wide, anteroposteriorly elongated supratemporal fenestra. The shelf of the fenestra is approximately three times larger than the fossae and extends especially posteriorly on the squamosal and medially on the parietal. The lateroventral margin of the maxilla is smooth and slightly concave (Pol 2003) that might suggest the attachment of a developed bucca here. The distal end of the quadrate has a ventral orientation. The quadrate condyles are unknown in all of the earlier Sphagesaurus specimens (Pol 2003, Andrade and Bertini 2008c) but well preserved on the left side of the skull of 'Caipirasuchus' (MPMA 670001/00) (Iori 2008, Iori and Carvalho 2011). The unusual medial condyle is rather processlike and extremely elongate ventrally (its ventralmost point is approximately $1 \mathrm{~cm}$ lower than that of the lateral condyle), and it is separated by a marked wide groove from the lateral condyle.

The occipital condyle is not preserved in any of the specimens.

The secondary bony palate is massive and narrow because laterally on both sides, the relatively wide alveolar margin of the upper teeth occupies its greatest part (Figure 29(B), 29(C)). Medially, the secondary palate possesses a shallow, sagitally oriented trough. In contrast to Mariliasuchus, the suborbital fenestra of Sphagesaurus is a huge, anteroposteriorly 
elongated opening bordered medially by the narrow palatines, posterolaterally by the rod-like part of the ectopterygoid, and anterolaterally by the lateromedially wide distal part of the ectopterygoid. Pol (2003:818) noted that a unique character of Sphagesaurus is the ,vertical wall located ventromedial to the orbital opening, running from the anterior margin of suborbital fenestra up to the orbital margin, where it contacts the jugal, lachrymal, prefrontal, and the anterior process of the pterygoids". Similarly to Mariliasuchus, the lateral pterygoid flange is narrow but distally it ends in a massive (ca. $2 \mathrm{~cm}$ thick) margin (Figure $30(\mathrm{C}-\mathrm{E})$ ). In lateral view, the pterygoid-ectopterygoid complex has an orientation of $40-45^{\circ}$ relative to the horizontal plane. The pterygoid-ectopterygoid complex of MPMA 15-001/90 is well extended laterally indicating the low capability of transverse movement of the mandibles The mandibular symphysis of RCL-100 (Pol 2003) and the more complete, anterior part of the mandible including a short postdentary part of MPMA 15-001/90 (Andrade and Bertini 2008a) have been published as Sphagesaurus (Figure 29(D-F)). In MPMA 67-0001/00 ('Caipirasuchus paulistanus' of Iori and Carvalho 2011) the almost complete right mandible including the articular region is preserved (Figure $30(\mathrm{G}, \mathrm{H})$ ). The mandible of Sphagesaurus is massive, especially at the level of the external mandibular fenestra, and has a lateromedially compressed, pointed symphyseal part. The anterior end of the completely fused symphysis is broken in both published specimens, but 'Caipirasuchus' shows that the symphysis is fused up to the level of the $7^{\text {th }}$ dentary tooth. Anteriorly, the postdentary part of the right mandible (MPMA 15-001/90) is very massive and elevated with large and anteroposteriorly elongated external mandibular fenestra. Dorsally, close to the dentary-surangular contact, the surangular is extremely wide (lateromedial width ca. $1 \mathrm{~cm}$ ) and robust perforated by some distinct foramina. In contrast to the massive anterior part, the posterior part of the mandible, from the middle region of the surangular, is more lightly built. The glenoid surface is covered with sediment, thus the extent and orientation of the glenoid surface cannot be properly defined, 
but in ventral view it is evident that this part of the articular is an anteroposteriorly elongated region. An unusual feature of Sphagesaurus is an oval-shaped, anteroposteriorly elongate opening (ca. 6-8 $\mathrm{mm}$ in length) anteroventral to the external mandibular fenestra within the splenial that can be observed on both MPMA 15-001/90 and MPMA 67-0001/00 (Figure 30(G)). Iori (2008) described this opening as „caudal intermandibular foramen”.

\section{Dentition and possible extant analogues}

Although the dentition of Sphagesaurus is well known, teeth are usually poorly preserved with crushed or damaged enamel coat and apical region, and in several teeth it is not clear if the eroded surface is due to wear or preservation (Figure 29(G)).

The dentition of Sphagesaurus resembles that of Armadillosuchus and Notosuchus because teeth are widely spaced, conical, and the posterior ones bear a single carina that is obliquely oriented relative to the sagittal plane. Individual posterior tooth morphology of Armadillosuchus is almost identical with that of Sphagesaurus. In several aspects, however, they are different from each other; most importantly in the exact orientation of the carina of the in situ upper teeth. Dentition of Notosuchus differs from those of Sphagesaurus and Armadillosuchus in the absence of longitudinal enamel grooves, and the orientation of the carina of the upper teeth is more distally oriented than in the two other genera. In addition, in sphagesaurids, both the upper and lower tooth rows, especially anteriorly, are closer to each other than in Notosuchus.

The number of premaxillary teeth appears to be different among the different species. The anterior end of MPMA 15-001/90 (Sphagesaurus montealtensis) is broken, thus the exact number of premaxillary teeth is unknown. In RCL-100 (Sphagesaurus huenei), Pol (2003) described an edentulous anterior end of the premaxillae that is followed by two premaxillary 
teeth. The first one is a large, distally curved caniniform tooth and the second one is not preserved in MPMA 15-001/90. In MPMA 15-001/90 the teeth directly behind the caniniform tooth are conical but poorly preserved. Andrade and Bertini (2008a) regarded these second upper teeth as the first maxillary teeth. However, in the new specimen ('Caipirasuchus paulistanus', MPMA 67-0001/00), the tooth behind the caniniform is still in the premaxilla (as in RCL-100), so I suggest that the second upper teeth of MPMA 15-001/90 are still premaxillary teeth. In addition, the new specimen (MPMA 67-0001/00) possesses two small, conical, slightly procumbent teeth anterior to the caniniform premaxillary tooth, so in this specimen, the premaxilla bears four teeth (Iori and Carvalho 2011, Figure 31(A)). In all species and specimens, six teeth are present in the maxilla and their morphology is basically similar (Figure 31(B)). The first four teeth are similar in size, the fifth is smaller than these and the last tooth is almost two times smaller than the anterior ones. The mesiolabial-linguodistally oriented conical tooth crowns have a drop-shaped cross-section, are ornamented by longitudinal enamel ridges as in Armadillosuchus, and they possess a single, linguodistally oriented carina. Although badly preserved in MPMA 15-001/90, this carina is tuberculated as in Armadillosuchus. The first and second contralateral maxillary teeth are still very close to each other (the distance between them is the size of one or two tooth). Posteriorly, from the third maxillary teeth, tooth rows become divergent (approximately $40-45^{\circ}$ relative to the sagittal plane).

Mandibular teeth are poorly preserved in the two more complete specimens (MPMA 15001/90, MPMA 67-0001/00) and the complete lower tooth row including 10 teeth is known only in the new specimen (MPMA 67-0001/00, Iori and Carvalho 2011). The lower tooth row can be divided into three segments among which the first four teeth are in the anterior part of the long symphysis (Figure $30(\mathrm{G}, \mathrm{H})$ ). These teeth are the smallest and the left and right rows run very close to each other. RCL-100 preserved four of the anterior symphyseal teeth but it is 
not clear if they represent the first four pair of teeth or the second to fifth pairs. These teeth increase in size posteriorly, they are conical with fine longitudinal ridges on the enamel coat and no carina has been reported on them (Pol 2003). The fifth tooth of MPMA 15-001/90 is the last in the straight symphyseal segment and it appears to have had a mesially oriented carina. The diastema anterior and posterior to the fifth tooth is the largest in the whole lower tooth row. Posterior to the fifth tooth, tooth rows become divergent similarly to the posterior segment of maxillary tooth rows. This segment is composed of five mesiolabiallinguodistally elongate teeth with drop-shaped cross-section, longitudinal enamel ridges and mesially-mesiolabially oriented carina. The sixth to eighth teeth are similar in size and the $9^{\text {th }}$ and $10^{\text {th }}$ are gradually smaller.

\section{Dental wear}

Due to the poor preservation of the teeth, dental wear can be detected only on a few of them. Scratch orientation and EDI cannot be observed in the more complete specimens (MPMA 15001/90, MPMA 67-0001/00); only the wear facets of RCL-100 provide some information (Pol 2003). The first two small anterior teeth of MPMA 67-0001/00 that are missing in RCL-100 (Sphagesaurus huenei) possess lingually positioned, flat wear facets with an angle of $40^{\circ}-50^{\circ}$ relative to the vertical plane. The largest, caniniform teeth bear developed, steeply inclined lingual wear facets (Pol 2003) similarly to Armadillosuchus. The last tooth in the premaxilla (second preserved in MPMA 15-001/90 and the fourth in MPMA 67-0001/00) bears steeply inclined (ca. $70^{\circ}-80^{\circ}$ relative to the horizontal plane) lingual wear facet and in MPMA 15 001/90 some apical abrasion can be also observed. Scratch orientation is unknown in the premaxillary teeth but the wear facets of the maxillary teeth and their scratches (Pol 2003) suppose a mesiodistal orientation. The obliquely oriented maxillary teeth possess wear facets on their distolingually positioned carina (Figure 31 ). The angle of wear facets relative to the 
horizontal plane apparently changed during the process of wear. In the early phase, wear facet is steeply inclined $\left(50^{\circ}-60^{\circ}\right)$ but as its area expands, the lingual wear facet becomes confluent with the apical wear and this enlarged wear facet is already closer to the horizontal plane $\left(30^{\circ}-40^{\circ}\right)$. The maxillary wear facets of Sphagesaurus are almost identical in form and orientation with those of Armadillosuchus. The mesial end of the scratches on these wear facets are „slightly more ventral” than their distal end (Pol 2003:818). In other words, these scratches have a mesiobasal-apicodistal orientation. The specimen RCL-100 exhibits the most eroded tooth (the right first maxillary tooth) among the Sphagesaurus specimens. This tooth possessing a simple, close to horizontal, extended wear facet is even more eroded than any teeth of Armadillosuchus and suggests that during dental occlusion, teeth became so eroded in their last stage that the labial, apical and lingual wear facets (as described for example in the 4th left dentary teeth of Armadillosuchus) united in a single wear facet.

Dental wear of the mandibular teeth is mostly on the labial or mesiolabial side of the tooth crowns forming the complementary surfaces of the maxillary wear facets. In the anterior symphyseal teeth of RCL-100, besides the labial wear facets, apical and small lingual wear facets also occur (Pol 2003), similarly to the dentary teeth of Armadillosuchus. This is particularly developed on the fourth teeth and here, the main orientation of parallel scratches is mesiodistal but some apicobasal scratches have been also described (Pol 2003). Posteriorly, the obliquely orineted dentary teeth bear developed, steeply inclined (ranging from $40-70^{\circ}$ ), roughly mesiodistally oriented labial wear facets on the tuberculate carina. In addition, both apical and lingual abrasions of the teeth occur.

\section{Adductor muscle reconstruction}

The main origin and insertion surfaces of the cranial adductor muscles are quite similar in all specimens of Sphagesaurus, thus a general reconstruction of the adductor musculature can be 
provided (Figure 32). Not surprisingly, along with the similar morphology of the dentition and dental wear, the reconstructed adductor musculature of Sphagesaurus strongly resembles that of Armadillosuchus. In Sphagesaurus, the palatine-pterygoid-ectopterygoid complex is preserved (in Armadillosuchus this region is missing), thus here the relative mass of pterygoid muscles can be also estimated. The huge suborbital fenestrae providing extensive muscle origin surfaces on the surrounding palatines, ectopterygoid, and maxilla clearly indicate that the MPTD was strongly developed (Figure 32(A)) in contrast to that seen in Mariliasuchus. This is further supported by the unique vertical maxillary wall located at the anterior margin of the suborbital fenestra that dorsally reaches the orbital margin (Pol 2003). This area is the posterior end of the nasal cavity, thus it is suggested here that this bony wall might have provided additional bony origin surfaces for MPTD (Holliday and Witmer 2007). In addition, based on the specimen described by Iori and Carvalho (2011), in the high postdentary part of the mandible, the dorsomedial process of the angular is massive and expanded suggesting important attachment area for MPTD. On the other hand, the relatively steeply inclined pterygoids are narrow and the posterior part of the mandible behind the external madibular fenestra is not as robust and expanded dorsoventerally as in Malawisuchus, Notosuchus or in modern crocodyliforms. These features suggest a significant but not particularly developed MPTV compared to extant forms (Iordansky 1964, Busbey 1989, Figure 32(A, C)). The ventral surface of the distal quadrate is smooth and no crest or any protuberance as additional muscle origin surfaces (e.g. Iordansky's muscle scars) can be observed. This denotes that the total mass of MAMP was comparable or less than that seen in extant forms. The quadratojugal is a thin rod running along the anterolateral margin of the quadrate and, in contrast to Armadillosuchus, no expanded part is present at the posterior corner of the laterotemporal fenestra. On the other hand, the anterodorsal part of the surangular is a wide and massive region of the mandible suggesting that this region was the attachment area of the 
really advanced MAMEM and MAMES. The strongly extended shelf-like margins of the supratemporal fenestrae and the wide and anteroposteriorly extended supratemporal fossae unambiguously indicate the great mass of adductors originating from here (Figure 32(B)). Among these, MAMEP could have been the most significant the orientation of which had an important anteroposterior component. Although the lataral surface or the postorbital process of the laterosphenoid does not show any particular crests or rugose surfaces, the extended bone suggests developed MPSS at least comparable to that of extant forms.

It is unknown, whether the anteriorly positioned medial openings on the splenials of Sphagesaurus had any functional role, for example as an attachment site of the cranial muscle system.

Comparison of the reconstructed cranial adductors of Sphagesaurus, Mariliasuchus and Armadillosuchus reveals that the external adductors (MAMEP, MAMEM, MAMES, or at least one of them and perhaps MPSS) were strongly advanced in all genera, whereas MPTV was much less developed and had minor role compared to that of extant forms. Based on the unusually expanded and crested origin surfaces, Armadillosuchus could have had the relatively most advanced MAMES and MAMEM among the three taxa. In contrast to Mariliasuchus, Sphagesaurus possessed a highly advanced MPTD.

\section{Jaw mechanism and possible phases of chewing}

Although several specimens of Sphagesaurus are available, the details of jaw mechanism and dental occulsion is not as unambiguous as in Mariliasuchus or Armadillosuchus mainly because of the poorly preserved teeth and wear pattern. Using cranial and dental features and characters of the wear pattern, Pol (2003) reconstructed a complex mandibular movement of Sphagesaurus that involved both anteroposterior and lateromedial components. The new remains (Andrade and Bertini 2008a, Iori and Carvalho 2011) further support the hypothesis 
of complex jaw mechanism and provide additonal details. Until the discovery of the newest, most complete specimen, the quadrate-articular contact of Sphagesaurus was unknown. In MPMA 67-0001/00 (Iori and Carvalho 2011), the right glenoid surface and the complete left quadrate with a ventrally unusally elongate, process-like medial quadrate condyle are preserved. This quadrate condyle morphology is extremely unusual among crocodyliforms. The glenoid surface was not fully prepared so the exact articulation of the quadrate on the glenoid surface is not completely clear, but the glenoid surface appears to be a relatively flat, horizontal and anteroposteriorly elongate surface that is strongly posteriorly positioned without buttress behind it. The question is what kind of functional consequences this unusually long, medial quadrate condyle could have had during mandibular movement? The ventralmost point of the medial condyle is approximately one centimeter below the ventral level of the lateral condyle, so both of the strongly asymmetrical quadrate condyles of MPMA 67-0001/00 cannot fit to the relatively flat glenoid surface. The lateral surface of the obliquely oriented, medial condyle is slightly convex so perhaps this condyle fitted not in but onto the medial side of the cartilagous part of the glenoid surface and could have been a medial sliding and rotation surface. The glenoid surface is bordered posteromedially by the short, posteromedially facing retroarticular process. It is conceivable that in contrast to MPMA 670001/00, the medial quadrate condyle of the other species of Sphagesaurus (RCL-100, MPMA 15-001/90) had a glenoid surface morphology that, similarly to other notosuchians with proal or palinal jaw movement, allowed a significant anteroposterior shifting of the mandibles as it was concluded by Pol (2003). Based on scratch length on the wear facets of RCL-100, the maximum anteroposterior shifting of the mandibles was about 1 centimeter (based on Pol 2003:fig. 3). On the other hand, dentition and wear facets of MPMA 15-001/90 and MPMA 67-0001/00 are obviously identical with that of RCL-100 (and at least the dental wear with those of Armadillosuchus), so there is no reason to suppose a different jaw 
mechanism for MPMA 67-0001/00. If there was indeed a difference in quadrate morphology among these Sphagesaurus specimens, then only the length of the anteroposterior component of mandibular movement could have been different.

Another important question is the precise direction of longitudinal mandibular movement during the powerstroke, namely that it was proal or palinal? This can be determined by the EDI and/or the orientation of oblique scratches. Teeth of two specimens (MPMA 15-001/90 and MPMA 67-0001/00) are too poorly preserved to recognize scratches on the wear facets (Figure 31(C, D)). In addition, Pol (2003) has not discussed the details of the EDI but he wrote that: "on the surface of the wear facets there are numerous parallel striae oriented anteroposteriorly, with their anterior end slightly more ventral than their posterior end" (Pol 2003:818). This orientation of the wear facet on a maxillary tooth suggests that the exact orientation of these scratches is mesiodistal and slightly apicomesial-distobasal. This shows that when the upper and lower teeth occluded, the mandibles were retracted (i.e. palinal movement existed) and moved posteriorly and slightly upward, roughly similarly to that of Armadillosuchus (Figure 33(A-D)). This is supported by the reconstructed advanced external cranial adductors, especially by the well developed MAMEP that had a significant anteroposterior component.

As it was pointed out by Pol (2003), an alternating jaw closure must have existed in Sphagesaurus because when the teeth occluded on the right side, those of the left side are not close enough to simultaneously produce tooth-tooth contact. This unambiguously indicates that jaw movement was completed by a lateromedial component (Figure 33(E-H)). Labiolingually oriented scratches, however, do not occur on any of the specimens, thus, similarly to Armadillosuchus, the lateromedial movement could not have played any role during occlusion but it was only essential for side switching dental occlusion. The maximum length of lateromedial movement in Sphagesaurus was shorter than in Armadillosuchus and 
this movement was most probably motorized by the pterygoid muscles. Regarding lateromedial movement of the mandibles, lateromedial translation or longitudinal rotation of the hemi-mandibles can be excluded. The former displacement was prevented by the unusually elongated medial quadrate condyle, whereas the latter by the deeply concave groove between the quadrate and the fused symphysis. This movement must have been carried out by rotation of the mandibles about a pivot point, and this point could have been in or close to the medial quadrate condyle. This rotation was allowed by the laterally weakly extended pterygoid-ectopterygoid complex.

Similarities of the dental morphology, the position and orientation of the wear facets, and the orthal jaw mechanism completed with palinal and lateromedial components suggest roughly similar phases of chewing between Armadillosuchus and Sphagesaurus, so they are not discussed here again. The only important difference between the two taxa are the absence of huge, procumbent first dentary teeth and the smaller premaxillary caniniform tooth in Sphagesaurus. These enlarged anterior teeth of Armadillosuchus most probably played an important role in digging and/or capturing larger prey (see the later chapter on the life habits of heterodont crocodyliforms).

Notosuchia Gasparini, 1971

Adamantinasuchus navae Nobre and Carvalho, 2006

(Figure 34)

Holotype-UFRJ-DG 107-R, incomplete skull and mandible, and postcranial material. Referred specimens-UFRJ-DG 216-R, incomplete skull with postcranial material (approximately with the same size as the holotype).

Type Locality—25 km southwest from the city of Marília, São Paulo State, Brazil (Nobre and Carvalho 2006). 
Type Horizon-Bauru Basin, Adamantina Formation, Upper Cretaceous (TuronianSantonian).

\section{General cranial and mandibular morphology}

Although the main part of the right side of the skull is preserved in the holotype, most parts of the temporal, palatal, and occipital regions and the whole jaw joint are missing or strongly damaged (Figure 34). The skull is slightly compressed lateromedially due to diagenetic events. The holotype skull could have been approximately six $\mathrm{cm}$ long, relatively high with extremely large, laterally facing orbits, and anteriorly facing external nares. The rostrum is relatively short; its anteroposterior length only slightly exceeds that of the orbit. The very lightly built skull with slightly disintegrated skull elements, the extremely huge orbit (approximately one third of the total skull length) and the absence of extensive abraded surfaces of the highly complex dentition (see below) strongly suggests that this specimen is from an ontogenetically immature animal. Ventrally, the orbits and the relatively small, triangular lateral temporal fenestra are bordered by an anteroposteriorly elongate, massive jugal the lateral margin of which is expanded and blade-like. Of the skull roof, only the right postorbital is preserved. Its posteromedial concave, smooth margin suggests a relatively expanded supratemporal fenestra (at least in this direction, Figure 34(B)), and the preserved right lateral, anterolateral wall of the braincase indicates a rounded supratemporal fossa with a diameter of approximately $1 \mathrm{~cm}$. The postorbital of Adamantinasuchus is very similar to that of Yacarerani (see below) which possesses an extremely developed supratemporal fenestra. Due to the lack of the parietal and squamosal, the posterior and medial extension of the supratemporal fenestra is unknown.

Nobre and Carvalho (2006) noted that the preserved right mandible has a strong bending in the longitudinal plane. Actually, this bending characterizes only the dentary that has a marked 
concave alveolar margin. This lateral alveolar margin has a lateromedially wide shelf. Of the postdentary bones, the anterior part of the surangular and the angular are preserved bordering the relatively large, anteroposteriorly elongate external mandibular fenestra dorsally and ventrally, respectively. The anterodorsal part of the surangular is wider than high. The mandibular symphysis is fused.

\section{Dentition and possible extant analogues}

Nobre and Carvalho (2006) shortly described the dentition of Adamantinasuchus, so a more detailed description with some additional comments related especially to the multicusped teeth is added here. Teeth of Adamantinasuchus are quite unique even among heterodont notosuchians. Similarly to Mariliasuchus, the anterior lower teeth and slightly the first upper one are strongly procumbent (Figure 34(D)). Teeth of Adamantinasuchus are relatively widely spaced, so the neigbouring crowns do not have contact with each other (Figure 34(E, F)). The premaxilla contains three slightly distally curved teeth among which the first two are smaller. The first premaxillary teeth are preserved on both sides. The left one is fully erupted, slightly procumbent and similar in size to the second tooth of the right side. Both of the anterior two teeth are conical, pointed and their enamel surface is ornamented by weak, longitudinal ridges. The second premaxillary tooth preserved on both sides bears a sharp and straight, unserrated carina distally-labiodistally. The largest third tooth is a hypertrophied caniniform with longitudinal enamel ridges. The lingual surface of these premaxillary teeth are neither cuspidate nor crenulate.

Maxillary teeth bear a developed carina on the lingual side of the crown. These carinae are oriented distolingually, and posteriorly they gradually have a more lingual (transverse) orientation. This feature strongly resembles tha carina morphology of the maxillary teeth of Armadillosuchus and Sphagesaurus. The first tooth in the maxilla appears to be the smallest 
in the upper tooth row being conical, pointed, slightly distally curved with a distallydistolingually oriented, unserrated carina. The second maxillary tooth is stockier than the anterior ones and has an oval or perhaps drop-shaped cross-section. Centrally on its lingual surface, it bears a distolingually oriented, unserrated, prominent carina that reaches the base of the crown. Posterior to this tooth, subsequent teeth are slightly constricted at their base. The third maxillary tooth is larger than the second one and in labial view its stocky crown is slightly distally curved. It has an oval cross-section at its base and on the obliquely facing, lingual surface it bears a developed, distolingually facing carina with 8 tubercles (Figure 34(E)). These tubercles are quite similar to those on the carinae of the posteiror teeth of Mariliasuchus. In addition, the distal part of the base of the crown bears a crenulate enamel margin but still no distinct tubercles or cusps can be seen. In a close-up view, the enamel surface is sligthly irregular. The fourth tooth is the largest in the maxilla having a relatively similar shape to the third tooth. The central, lingually-distolingually oriented carina is not tuberculate but rather cuspidate possessing at least 6 cusps (the labially positioned tip of the crown is covered with sediment). These cusps are almost as wide labiolingually as mesiodistally, and they are bordered by grooves that run mesially and distally to the base of the crown. The mesial and distal margins of the tooth crown are tuberculated. The fifth tooth is slightly smaller than the fourth but possesses an almost identical morphology with 8 cusps on the almost lingually facing carina. Similarly to the previous teeth, the sixth maxillary tooth has a labiolingually wider, oval shape, it is two times smaller than the fifth tooth, bears a tuberculate, lingually oriented carina on its obliquely facing lingual surface, and its mesial and distal margins are also finely tuberculated. The last, seventh tooth is the smallest, it has a massive, lingually orineted, tuberculate carina on its obliquely facing lingual surface, but the mesial and distal margins are devoid of tuberculate carinae. Only little information is avaiable 
on the replacement teeth. The proximal end of the barrel-like roots of the fourth to sixth teeth are open but no indication of replacement teeth can be seen.

The mandibular tooth row is poorly known. Only some teeth are preserved in the anterior region but it is not completely clear which positions they represent because the lower jaw is articulated to the skull and this anterior region is still covered with matrix. The first dentary teeth, preserved on both sides, are strongly procumbent and almost horizontally oriented. These teeth are conical (the apical half of the left one is broken), and similarly to that of the premaxillary teeth, the enamel surface is ornamented by weak, longitudinal ridges. The first dentary teeth are interdigitating with the first and second premaxillary teeth (Figure 34(D)). Due to the matrix and preservation, only a procumbent tooth base can be seen of the next (perhaps second or third) dentary tooth in medial view but this tooth is not as anteriorly oriented (approximately $45^{\circ}$ ) as the first tooth. The following tooth is still slightly procumbent and pointed with a smooth lingual surface. Its labial surface cannot be observed. The last preserved tooth of the right dentary is in a close, complementary position with the third maxillary tooth. It is conical and bears a labially-mesiolabially oriented carina on its obliquely facing labial surface (Nobre and Carvalho 2006). Posteriorly, the labial margin of at least three additional alveoli can be observed on the crushed dentary which most probably accomodated teeth similar to the posterior maxillary teeth but with a complementary morphology (i.e. mesiolabially-labially oriented, tuberculate carina).

\section{Dental wear}

The teeth of Adamantinasuchus bear only a few wear facets predominantly on the anterior teeth but they are also present on the posterior cuspidate teeth (contra Nobre and Carvalho 2006). Unfortunately, due to the delicate preservation of the specimen and the covering conservation material, I was not able to take molds from the teeth to see the details of the 
wear pattern. In spite of that, these wear facets also provide useful information. The labial surface of the anterior two premaxillary teeth bears circular or oval-shaped, almost vertically positioned wear facets that are clearly the result of the contact with the procumbent anterior dentary teeth. Similar but much more extensive wear facets are present in Armadillosuchus. Besides the labial wear facets, apical wear also occurs on these teeth, for example, on the tip of the left premaxillary teeth. Concerning the anterior dentary teeth, the first right one bears an apical wear on its ventral-labioventral surface. It resembles the ventrally positioned extensive apical wear of the similarly procumbent, anterior dentary teeth of Mariliasuchus.

A single, but clearly identifiable wear facet can be recognized on the cuspidate lingual carina of the largest, fourth maxillary tooth (Figure 34(F)). This small, labiolingually elongate and obliquely facing wear facet is formed by the abrasion of at least three cusps on that carina. The abrasion did not reach the dentine but the original enamel cusps became deeply eroded. Unfortunately no detailed morphology of this wear facet (EDI, scratches, pits) can be seen. The low degree of abrasion especially on the posterior cuspidate teeth are perhaps related to the early ontogenetic stage of this specimen of Adamantinasuchus suggested earlier, and perhaps the juveniles of Adamantinasuchus fed on softer food than adults. It is well known for example in Crocodylus johnstoni (Tucker et al. 1996) and in Caiman latirostris that adults consume snails, fish, birds and mammals, whereas juveniles are predominantly insectivorous (Brito et al. 2002).

\section{Adductor muscle reconstruction}

Since the palatal region including the pterygoid-ectopterygoid-suborbital fenestra complex is not preserved, and the posterior end of the mandible including the insertion surfaces for MPTV on the angular and below the articular are crushed, the relative mass of the pterygoid muscles cannot be estimated. Although the posterior part of the temporal cavity is also 
crushed, some information is still available on the origin and insertion surfaces of the external adductors. The supratemporal fenestra appears to have been well developed because it extends well anteriorly on the postorbital. This suggests a relatively advanced MAMEP (Figure 34(B)). The quadrate-quadratojugal complex is also crushed, so the origin surfaces of MAMP, MAMEM and MAMES cannot be studied. Contrary to the huge orbits, the lateral temporal fenestra is small. If the holotype specimen represents an immature specimen, then the rostrum should have been slightly longer and the orbits relatively smaller in adults. These are well documented changes during ontogeny of crocodylians (Kälin 1933, Iordansky 1964). Furthermore, adults must have possessed a larger supratemporal fenestra, and with the expansion of the width of the postorbital cranial roof (Iordansky 1964), the lateral temporal fenestra was possibly also larger to accomodate a larger mass of external adductors. The postdentary part of the mandible is slightly elevated and the surangular is wide lateromedially, so it can be assumed that the MAMEM and MAMES were comparable in mass to that seen in extant crocodylians.

\section{Jaw mechanism and possible phases of chewing}

The very restricted information on tooth wear pattern, the lack of quadrate-mandible articulation, and the weak estimation of the relative mass of jaw adductors prevent a detailed reconstruction of the jaw mechanism in Adamantinasuchus. Nonetheless, the available feeding-related characters provide a high number of data to outline a possible mechanism of dental occlusion and mandibular movement in this animal. The completely fused and elongated mandibular symphysis, for example, indicates that both mandibles moved synchronously, and there was no axial rotation of the hemi-mandibles, similarly to Mariliasuchus and sphagesaurids. The strongly procumbent anterior dentary teeth with their interdigitating position with the conical premaxillary teeth along with the large, third 
caniniform tooth in the premaxilla (Figure 34(D)) show that when the mandibles were closed or almost in a closed position, the fused mandibles could not rotate or slide transversely to produce lateromedial movement, so the main type of jaw closure was orthal. Tooth morphology and the wear facet on the fourth right multicusped maxillary tooth unambiguously indicate that dental occusion of the posterior cheek teeth was characteristic for Adamantinasuchus and possibly the orthal movement was complemented by some anteroposterior jaw movement. The distolingually and lingually oriented, obliquely positioned carinae of the maxillary teeth that bear cusps from the third tooth could have been perfect tools to come into an antagonistic, shearing contact with those of the lower teeth. This could have been especially efficient if orthal movement was accompanied by an anteroposterior movement. The question is whether the powerstroke was characterized by proal or palinal mandibular movement. Unfortunately, no direct evidence (e.g. orientation of scratches, EDI) can show the direction of movement during occlusion but some craniodental features and taxonomic implications may help to answer this question. First of all, the general morphology of the maxillary and dentary teeth and the orientation of their carinae are strongly similar to that of Armadillosuchus or Sphagesasurus, taxa where palinal mandibular movement was demonstrated. Second, the preserved margin of the supratemporal fenestra on the postorbital suggests a large supratemporal fenestra that is an indicator of a highly developed MAMEP and has been interpreted to act as one of the motors of mandibular retraction (i.e. palinal movement). Third, a recently described highly specialized notosuchian, Yacarerani from the Late Cretaceous of Bolivia (see below) was described as the sistertaxon of Adamantinasuchus (Novas et al. 2009). Yacarerani has a dentition with multicusped teeth (although the cuspidate rows of the crowns have an almost mesiodistal orientation) quite similar to Adamantinasuchus and it possesses a strongly expanded supratemporal fenestra that was also supposed in Adamantinasuchus. These features strongly suggest that in 
Adamantinasuchus, the ortal jaw closure was complemented in the powerstoke by a palinal movement of the mandibles in which the fused lower jaws moved backwards and perhaps upwards, generally similar to that of Mariliasuchus, Yacarerani, and sphagesaurids.

The function of the procumbent anterior teeth could have been similar to those of Mariliasuchus including strong biting probably repeated several times in the preparatory stroke, in a similar manner described in rodents (Weijs 1975, Gorniak 1977) and reconstructed in multituberculates (Gambaryan and Kielan-Jaworowska 1995). These teeth were used to capture food with a grasping and holding function. In addition, similarly to Mariliasuchus, Adamantinasuchus could also have used its anterior teeth to burrow which idea is supported by the labial (outer) wear on these teeth and also by sedimentological and taphonomical evidences (see below).

The phases of oral food processing is not discussed here in detail because available evidence is not sufficient to unequivocally clarify the mechanism of jaw movement and the process of dental occusion of the multicusped teeth. Nevertheless, if the hypotheses outlined above (i.e. the presence of dental occlusion and orthal jaw closure complemented with a retractive powerstroke) are correct, the phases of chewing were basically similar to other crocodyliforms with palinal jaw movement (i.e. Mariliasuchus, sphagesaurids, see Figure 25).

Notosuchia Gasparini, 1971

Yacarerani boliviensis Novas, Pais, Pol, Carvalho, Scanferla, Mones and Riglos, 2009 (Figure 35, 36)

Holotype-MNK-PAL5063, complete skeleton including skull and mandibles. Referred specimens-MNK-PAL5064 skull fragment with articulated mandibles, postcranial material. 
Type Locality-Amboro National Park, Santa Cruz de la Sierra, Bolivia (Novas et al. 2009). Type Horizon - Cajones Formation, Upper Cretaceous. The more precise age of the formation is controversial. It was interpreted as Maastrichtian on the basis of the occurrence of two fish taxa (Pucapristis branisai and Gasteroclupea branisai; Lopez 1975, Aguilera et al. 1989) that were documented only from Maastrichtian sediments of South America. Based on the close relationship of Adamantinasuchus and Yacarerani and on sedimentological similarities of the Adamantina and Cajones Formations, however, Novas et al. (2009) inferred a TuronianSantonian age. If Yacarerani is indeed from the Maastrichtian then it is the latest representative of heterodont notosuchians in South America. (Besides Yacarerani, only Simosuchus from Madagascar is from the Maastrichtian).

\section{General cranial and mandibular morphology}

The skull of Yacarerani is compact and oreinirostral. The rostrum is very short being approximately one third of the total skull length. Skull elements are strongly fused with unpaired frontals and parietals indicating an adult or close to adult ontogenetic stage of the type specimen. The skull becomes narrow just anterior to the huge, laterally-anterolaterally facing orbits (Figure 35(A)), similarly to Mariliasuchus. The large external nares are confluent and facing anteriorly. The nasals overhang the external nares, and anteriorly, their dorsal surface is ornamented by numerous small pits which suggest a sensitive circumnarial region. The skull roof or Yacarerani is highly modified. Approximately $80 \%$ of the skull roof is occupied by the extremely extended supratemporal fenestrae. The shelf of these openings extends onto most of the parietal, squamosal and postorbital. As a consequence, the characteristic ornamentation of the crocodyliform skull roof can only be observed on the anterior edge of the parietal, in a median ridge of the skull roof, and in the posterolateral corner of the squamosal (Figure 35(A)). Even the posterior and lateral parts of the postorbital 
are smooth and shelf like, similarly to Adamantinasuchus. The supratemporal fossa is large and rectangular, similarly to that of Mariliasuchus. The lateral temporal fenestra is large, triangular and bordered anteriorly by a narrow postorbital-jugal bar and lateroventrally by a weak and slender lower temporal bar. The robust distal part of the quadrate is vertically oriented (Novas et al. 2009) and more extened ventrally than that of Mariliasuchus. Novas et al. (2009:1316) noted on the quadrate condyles that ,the medial one is more acute and ventrally more projected than the lateral one". The occipital condyle is oriented posteriorly and slightly posteroventrally. The ventral basis of the short and narrow rostrum is formed by a massive secondary palate composed of the premaxillae, maxillae and palatines. The anteriorly expanded and fused ends of the palatines form a sagittal crest of approximately $1 \mathrm{~cm}$ length. Posteriorly, the secondary bony palate is rimmed by two pairs of large openings. The secondary choana is a large, deltoid-shaped, divided opening emarginated laterally by the columnar palatines and posteriorly by the pterygoids. Lateral to the secondary choana, the triangular suborbital fenestrae are comparable in size with that of Mariliasuchus. The ectopterygoids bordering laterally the suborbital fenestrae are massive elements. The pterygoid-ectopterygoid-palatine contact is a lateromedially wide, massive junction (Figure 35(B)). The pterygoid is short and robust, and similarly to Mariliasuchus, it does not bear an extended lateral pterygoid flange in contrast to that seen in modern crocodylians. Laterally, the pterygoid ends in a robust knob-like margin (ca. $1.5 \mathrm{~cm}$ long anteroposteriorly). The mandible of Yacarerani is unusual in various aspects. The mandibular symphysis formed by the dentaries and splenials is massive and strongly elongate reaching posteriorly to the level of the posterior maxillary teeth, but it is not ossified as for example in sphagesaurids. Because this symphyseal region contains numerous teeth, it can be supposed that in vivo the two hemi-mandibles were strongly connected to produce efficient oral food processing. The anterior half of the symphyseal region is straight and behind the third mandibular teeth, the 
dentaries become abruptly divergent and they are convex laterally. The postdentary part bears a large, oval-shaped external mandibular fenestra. No coronoid eminence is present at the dentary-surangular contact but instead this region is wide and flattened (Novas et al. 2009: fig. 2D). The jaw joint is in the level of the occlusal plane. The glenoid surface is anteroposteriorly elongate (approximately two times longer than the anteroposterior width of the quadrate condyles) and no buttress is present between the glenoid surface and the retroarticular process. The retroarticular process is extremely short but similar to Notosuchus, Mariliasuchus and Sphagesaurus (Iori and Carvalho 2011) in having a posteromedially sloping dorsal surface. Similarly to Sphagesaurus, a circular opening (ca. $5 \mathrm{~mm}$ in diameter) is present on the splenial at the level of the last dentary teeth (Figure 35(B)).

\section{Dentition and possible extant analogues}

The dentition of Yacarerani is beautifully preserved in both the upper and lower jaws and shows an extreme heterodonty with procumbent, conical anterior and multicusped posterior teeth (Novas et al. 2009). Dental morphology of Yacerarani shows that the oral cavity is divided into a narrow anterior and a triangular-shaped, posterior region by the fifth tooth pairs of the upper and lower jaws (Figure 36(E-H)).

The premaxilla contains four conical teeth among which the third is the largest (11 mm long), the first is medium-sized ( $7 \mathrm{~mm}$ long), and the second and fourth are the smallest ( $3-4 \mathrm{~mm})$ (Figure 35(E)). The first premaxillary tooth is strongly procumbent, slightly distally curved and is void of any enamel wrinkles or carinae. The second tooth is stockier than the first tooth but it is also procumbent and devoid of enamel wrinkles or carinae. The third, largest tooth corresponds to the caniniform of the tooth row. It is still slightly procumbent, gently distally curved, and its enamel surface is smooth. The fourth tooth is labiolingually slightly compressed, distally curved and bears a distally-linguodistally oriented carina. 
As it was noted by Novas et al. (2009), the premaxillary series is followed by a diastema and then by six oval-shaped, mesiodistally elongate maxillary teeth with complex morphology that form a strongly divergent (approximately $40^{\circ}$ relative to the sagittal plane) part of the tooth row. These teeth are closely packed but do not have contacting areas. Due to the divergent orientation of the tooth row, the subsequent teeth are labiodistally positioned relatively to each other. Unusually, the contralateral first maxillary teeth are situated very close $(2-3 \mathrm{~mm})$ to each other thereby forming the posterior border of the anterior part of the divided oral cavity. This tooth pair is smaller than the more posterior ones and it possesses a central, distally oriented, cuspidate carina extending from the apex to the base of the crown. On the distal half of the crown, smaller, cingulum-like rows of tubercles are present both labially and lingually. Based on photographs taken of the type specimen, labial to this tooth, the apex of a replacement tooth can be observed. The second maxillary tooth is much larger with a triangular shape in lateral view and has a mesially positioned apex. On the subsequent maxillary teeth, the distally positioned carina becomes a row of cusps. The central row of cusps has a distal, slightly linguodistal orientation with at least seven cusps. Labially and lingually the tooth crowns are bordered by marginal rows of cusps which also have a distal, slightly linguodistal orientation. In all maxillary teeth, these marginal rows of cusps are slightly bowed following the convex margin of the crown. The third to sixth maxillary teeth are roughly similar in morphology to the second one, although the fifth and sixth are smaller. These teeth also bear a distally or slightly linguodistally oriented surface with three rows of cusps (Figure 35(B)). The rows contain at least six to nine cusps.

The mandibular tooth row is quite similar to the upper one in having four conical teeth anteriorly that are aligned in a mesiodistal row and separated by a $3 \mathrm{~mm}$ long diastema from the rest of the lower tooth row. In addition, the posterior segment of the dentary tooth row including six, multicusped teeth is also divergent posteriorly, similarly to the upper tooth row 
(Figure 36(E)). The first dentary tooth is the largest in the mandibular tooth row, it is strongly procumbent, conical and slightly curved upwards. It is devoid of any enamel wrinkles but a shallow carina is present labially. The second to fourth teeth are smaller conical teeth that are slightly posteriorly curved. The first multicusped (fifth) dentary tooth is situated even more medial than the anterior conical teeth, so the fifth contralateral dentary teeth are even closer to each other than the first molariform teeth of the upper tooth row. The fifth right dentary tooth bears a mesiolabially oriented cuspidate carina, and on its distal side a marginal row of cusps. The following three teeth (sixth to eighth) in the dentary are approximately 1.5 larger than the fifth tooth and on the mesial surface, they bear a central row of cusps and lingually and labially one-one marginal row of cusps. The number of cusps varies from 6 to 9 . These rows are aligned in a roughly mesiodistal direction, so these teeth show the complementary morphology of the upper, multicusped teeth. The last two dentary teeth are smaller with the last one being the smallest in the row. They also bear rows of cusps (the last tooth has only two short rows) that are slightly curved and oriented mesially-mesiolabially.

\section{Dental wear}

Wear facets on the teeth of Yacarerani are present both on the anterior, conical teeth and also on the multicusped posterior teeth. Unfortunately, I was not able to study the specimens personally, thus no detailed dental wear analysis is available in this specimen. The observations presented here are based on the figures of Novas et al. (2009) and the photograps seen in Figure 36. The most frequent type of wear on the anterior teeth is apical wear that can be observed on the first three premaxillary teeth but it is also present on the first right dentary tooth. In the latter case, almost the third of the apical tooth crown became eroded and the largest wear facet is facing labially and slightly upward (Figure 35(E)). Furthermore, the left premaxillary tooth bears an extensive wear facet on its labial (outer) surface quite similar to 
those of Mariliasuchus. Some apical wear can be also seen on the fourth premaxillary tooth and also on the second and fourth conical dentary teeth. On the multicusped teeth of the type specimen, extensive wear can be seen on the first right maxillary and dentary multicusped teeth (Novas et al. 2009:fig. 3A, B) that are apparently complementary surfaces of each other. The wear facet of the maxillary tooth extends along the central, cuspidate carina from the apex towards the base of the crown, so it is facing distally and ventrally. Apically it is much wider labiolingually than basally and the dentine is widely exposed. The complementary wear facet of the fifth dentary tooth (Figure 36(E)) is similar in shape to that on the maxillary tooth and it is facing mesiolabially and dorsally. Up to the present, no information is available on the scratches, pits and EDI of any of the wear facets.

\section{Adductor muscle reconstruction}

The main jaw adductors and their relative mass can be reconstructed and estimated in Yacarerani because the temporal region of the skull and the postdentary part of the mandible are well preserved. The general architecture of the skull and mandible is quite similar to that of Mariliasuchus (Novas et al. 2009), so an adductor musculature similar to that reconstructed in Mariliasuchus is expected. As in Mariliasuchus but in contrast to extant crocodylians, the pterygoids are strongly reduced indicating a poorly developed MPTV (Figure 35(B)). Compared to Mariliasuchus, the rostrum of Yacarerani is narrower, the suborbital fenestrae are more anteriorly positioned and relatively smaller, the secondry choana is much larger and anteriorly extended, and the palatines are only columnar. This suggests a smaller mass of MPTD in Yacarerani than in Mariliasuchus. The ventral surface of the distal quadrate bears a prominent, almost $2 \mathrm{~cm}$ long crest extending from the basisphenoid-pterygoid contact posterolaterally. This crest corresponds to the crest B of Iordansky (1964) and similarly to extant forms it could have been served as the origin surface for the tendinous attachment of 
MAMP. (In Mariliasuchus crest B is missing but the crest A of Iordansky [1964] is present.) This indicates a well developed MAMP in Yacarerani, proportionally comparable to that of extant forms. The difference in crest morphology on the ventral surface of the quadrate between Mariliasuchus and Yacarerani, however, suggests some difference in the attachment of MAMP. Regarding the external adductors, they were highly developed. The elongate dorsal surface of the postdentary part of the mandible including a ,flattened area in the dentary-surangular contact" (Novas et al. 2009:fig. 2) reflects an extended insertion area for MAMES and MAMEM. The supratemporal fossae and fenestrae of Yacarerani are extremely large (the fenestrae are close to $80 \%$ of the skull roof, Figure 35(B)), thus the external adductors originating here (e.g. MAMEP) must have been highly developed and their orientation had a great anteroposterior component, similarly to Mariliasuchus and sphagesaurids. The same can be suggested in the case of MPSS, although the ventral part of the supratemporal fossa and the lateral, anterolateral surfaces of the laterosphenoid are still covered with sediment thus these surfaces cannot be observed.

Summing up the available information on the jaw adductors of Yacarerani, it can be concluded that the contrast between the pterygoid muscles (internal adductors) and external adductors in Yacarerani is even larger than in Mariliasuchus. The pterygoid muscles became even more reduced, and at least some parts of the MAME originating from the supratemporal fossa, lateral wall of the braincase, and supratemporal fenestra were even more developed.

\section{Jaw mechanism and possible phases of chewing}

The short and high rostrum, the divided oral cavity, the mesiodistally elongate, multicusped teeth with a complementary arrangement of the cuspidate surface on the upper and lower teeth, the wear facets, and the highly modified jaw adductors (based on MNK-PAL5063) unambiguously indicate that Yacarerani was one of the most specialized crocodyliforms 
regarding the degree of adaptation to oral food processing. Although the cranial, mandibular and dental morphology differs in many aspects from that of Mariliasuchus, the above listed features and their consequenses (such as a sophisticated dental occlusion, efficient oral food processing and an orthal jaw closure complemented with retractive powerstroke, i.e. palinal movement) easily bring the two taxa functionally close to each other.

Precise dental occusion is well supported by the complementary tooth morphology and the complementary wear facets of the right first maxillary and fifth dentary teeth (Figure 36(E, F)). These labiolingually oriented facets are relatively steeply inclinded, roughly parallel with the sloping, cuspidate surface of the crown indicating both vertical and longitudinal components of the mandibular movement. In addition, these wear facets are not found on the marginal cusps but the highest, central row of cusps bears the eroded surface. This shows that these anterior and probably also the posterior teeth of the upper and lower jaw occluded by the central rows of cusps. During this process, tooth occusion was not interdigitating (Figure $36(G))$ as seen for example in some teiids (Nydam and Cifelli 2002) and polyglyphanodontines (Nydam and Cifelli 2005). However, the existence of an additional interdigitating occlusion cannot be excluded. Based on the mesiodistal extension of the wear facet on the right first maxillary and fifth dentary teeth, the mandible moved at least $2-3 \mathrm{~mm}$ anteroposteriorly. The first maxillary tooth is, however, approximately two times shorter mesiodistally than the following maxillary teeth, so in the case of the more posterior teeth, the longitudinal component could have been 5-6 $\mathrm{mm}$. This is supported by the anteroposteriorly elongate glenoid surface that lack a buttress behind it. This 5-6 $\mathrm{mm}$ longitudinal movement, being almost the mesiodistal length of the largest multicusped teeth of Yacarerani, is relatively long and indicates the occlusion of quite large tooth crown surfaces. Although scratch orientation on the wear facets is unknown, the orientation of the cuspidate rows of the crowns, and the position, orientataion and morphology of the wear facets unambiguously 
indicate that when the upper and lower multicusped teeth occluded, the mandible was pulled upwards and at the end of a chewing cycle slightly backwards (Figure 36(A-D)). Similarly to Mariliasuchus, Armadillosuchus and Sphagesaurus, the existence of a palinal movement is strongly supported by the architecture of the muscle origin and insertion surfaces. Whereas the pterygoid muscles (especially the MPTV) are relatively reduced compared to those of extant crocodylians, the external adductors (especially the MAMEP) were highly developed. These muscles had a great anteroposterior component to pull the lower jaws backwards during occlusion (Figure 36(H)) and were responsible for a highly efficient food processing. The high efficiency of intraoral food processing is further demonstrated by the ratio between the extension of the temporal region where the adductors originated and that of the oral cavity. The latter is so narrow small compared to the the temporal region that the relative bite force in Yacarerani could have been one of the strongest among heterodont crocodyliforms. An unusual feature of the oral cavity of Yacarereani is the constricted position of the first maxillary teeth and the strongly divergent orientation of the tooth row behind it. The main consequence of this dental feature is that when the mouth was closed, the anterior part of the oral cavity with conical and mostly procumbent teeth was practically separated form the relatively small, triangular, posterior part with multicusped molariform teeth (Figure 36(G)). This indicates two, well distinguished phases during feeding as well.

1) Based on tooth morphology, the preparatory stroke could have been quite similar to that reconstructed in Mariliasuchus, so all details of this phase are not repeated here. The anteriorly positioned, procumbent teeth helped to capture the food i.e. had a grasping and holding function. Similarly to Mariliasuchus and sphageaurids but in contrast to multituberculates (Gambaryan and Kielan-Jaworowska 1995), the lack of lingually positioned wear facets indicates that these teeth did not have a gnawing function. In this phase, capturing and rough preparation of the food was completed perhaps in a few cycles. At the end of the 
preparatory stroke, food was transported into the posterior section of the oral cavity but apparently this could have happened only when the mouth was open. Wear facets on the labial (outer) surface of the procumbent anterior teeth, similarly to those of Mariliasuchus and sphagesaurids, indicate a burrowing habit of Yacarerani (see below).

2) During the powerstroke (Figure 36(A-D)), after food had been transported into the triangular shaped posterior region of the oral cavity, it was triturated between the complementary surfaces of the occluding, multicusped teeth. In this phase of food processing, the repeated orthal jaw closure was complemented by the palinal movement (retractive powerstroke) motorized dominantly by the external adductors. Tooth morphology and the wear facets show that the backward movement had not only been performed in the horizontal plane after the jaws were closed but this component of movement complemented the end of closing phase, thus producing the obliquely (mesioapically-distobasally on the upper teeth and mesiobasally-distoapically on the lower teeth) oriented wear facets, similarly to Mariliasuchus.

Difference in dental morphology between Mariliasuchus and Yacarerani, however, suggests some difference in the process of triturating the food. Whereas the oral cavity is confluent in Mariliasuchus, it is divided into a narrow anterior and a wide, triangular posterior block in Yacarerani. (the tongue of the animal certainly did not reach the anterior teeth when the mouth was closed because the left and right anterior lower teeth almost contacted each other). The reason, why this posterior part of the oral cavity is separated from the anterior segment in Yacarerani is still unknown but presumably this feature belongs to the most elaborate functional adaptations of oral food processing ever seen in a crocodyliform.

Notosuchia Gasparini, 1971

Candidodon itapecuruense Carvalho and Campos, 1988 
(Figure 37, 38)

Holotype-isolated tooth, (MN 4154-V, Carvalho and Campos, 1988).

Referred specimens - complete skull and postcranial material (UFRJ DG 114-R, Nobre and Carvalho 2002), left fragmentary dentary and postcranial material (UFRJ DG 113-R, Nobre 2004), fragmentary mandible and isolated teeth ( $\mathrm{MN} 4355-\mathrm{V}, \mathrm{MN} 4152-\mathrm{V}, \mathrm{MN} 4153-\mathrm{V}$, Carvalho 1994).

Type Locality_-Itapecuru Mirim, Parnaíba Basin, Brazil (Carvalho and Campos, 1988, Nobre and Carvalho 2002).

Type Horizon-Itapecuru Formation, Early Cretaceous (Albian).

\section{General cranial and mandibular morphology}

With a skull length of $7.8 \mathrm{~cm}$, Candidodon is one of the smallest genera among the heterodont notosuchians and rather comparable in size with other Early Cretaceous forms such as Malawisuchus and Pakasuchus. The gross morphology of the skull of Candidodon is similar to that of Sphagesaurus in having a narrow, oreinirostral skull with short rostrum, anteriorly facing external nares and laterally-anterolaterally facing, large orbits (Figure 37). However, in various aspects, the two crocodyliform differ from each other. The supratemporal fossa is relatively smaller and the shelf of the anteroposteriorly elongate supratemporal fenestra is less extended than in Sphagesaurus (or in Armadillosuchus and Mariliasuchus). This shelf is mainly extended medially onto the parietal and posteriorly onto the squamosal. The supratemporal fossa is positioned very close to the postorbital corner of the skull roof, similarly to that of Malawisuchus, thus, in spite of the extended shelfs medially, the sculptured parietal forms a great part of the central skull roof. The laterotemporal fenestra is trianguar and much smaller than the orbits, in contrast to that seen in Armadillosuchus. 
Candidodon possesses a medium-sized (ca. $5 \mathrm{~mm}$ in diameter), triangular antorbital fenestra. Of the short quadrates, the left one is complete with posteroventrally oriented lateral and ventrally oriented medial condyles. The lateral condyle is lateromedially wider and rounded ventrally, the medial one is pointed medioventrally. The two condyles are separated by a shallow and wide intercondylar groove (Figure 37(E)). The occipital region is poorly preserved, thus the orientation of the fragmentary occipital condyle is not clear. The ventral surface of the quadrate shaft is smooth and only a shallow crest occurs close to the pterygoidbasioccipital contact.

The secondary bony palate is wide and massive, bordered laterally by the straight but slightly divergent, narrow alveolar margins. The anteroposteriorly elongate suborbital fenestra is large bordered medially by the palatines and laterally by the massive and posteroventrally oriented ectopterygoids, so the fenestra is oriented actually slightly posteroventrally. The anteroposteriorly elongate palatines are fused sagittally forming a ridge on their ventral surface, and laterally they curve upward in a deep vertical wall to form the lateral wall of the secondary choana. Although the lateral pterygoid flanges are relatively not as wide anteroposteriorly as those of the extant forms, they are massive, elongate, and in posterior view, they are steeply inclined with an angle of approximately $50^{\circ}$ relative to the horizontal plane (Figure 37(E)). As usual in crocodyliforms, the pterygoid flanges end laterally in a massive and thick (ca. $6 \mathrm{~mm}$ anteroposteriorly) margin.

Of the mandibles, only a $1.5 \mathrm{~cm}$ long, left dentary fragment (in UFRJ-DG 113-R) is preserved but medially it is covered with matrix, so it is not clear if the symphysis was completely fused or not. In addition to this specimen, Carvalho (1994) described some poorly preserved anterior mandible fragments ( $\mathrm{MN} 4355-\mathrm{V})$.

\section{Dentition and possible extant analogues}


Teeth of Candidodon are best known in the skull of UFRJ DG 114-R (Nobre and Carvalho 2002). Some elements of the lower dentition have been best described based on a fragmentary mandible (MN 4355-V, Carvalho 1994), but isolated teeth are also known (Carvalho and Campos, 1988, Carvalho 1994). Some teeth are also available in a fragmentary dentary (UFRJ DG 113-R) but they are covered with matrix so the morphological details cannot be seen. The dentition of Candidodon differs from that of all other heterodont crocodylifoms discussed above. The upper tooth row can be divided into smaller to medium-sized, anterior fang-teeth, a large caniniform and smaller cheek teeth posteriorly (Figure 37(C)). Although it is not so pronounced in the anterior dentition, teeth of Candidodon are labiolingually compressed and carinated. The premaxilla contains most probably four or perhaps five alveoli, three of which bear teeth on the right side. The first preserved tooth is small, pointed, triangular, labiolingually compressed and carinated but the carinae are non-serrated. It is not clear if the following small gap between this tooth and the largest premaxillary tooth is simply due to preservation or it is an additional alveolus. The next and apparently penultimate tooth in the premaxilla is a large, triangular, labiolingually flattened tooth with non-serrated carinae mesiodistally. The last premaxillary tooth is smaller than the previous one and more pointed with mesiodistally oriented carinae. No serration can be observed on this tooth. Nobre and Carvalho (2002) described that the first two teeth in the maxilla are small and the crown is spatulated. Their figure (fig.1) and personal studies, however, do not reveal the first maxillary tooth on either side, so its morphology is unknown. The second maxillary tooth is preserved on the left side. It is a small, lanceolate tooth, labiolingually slightly compressed with mesiodistal carinae. Although the tooth is slightly worn, the serration of the distal carina can be observed. The third pointed maxillary tooth is the largest in the row, being a triangular, labiolingually compressed caniniform (Figure 38(A, B)). Both of these teeth are preserved, although the right one was broken off. Close-up views of the carine of these teeth revealed 
some fine serration mesiodistally that are hard to discern due to the wear pattern of the crowns. The caniniform is followed by a short $(4-5 \mathrm{~mm})$ diastema and then by four, closelypacked small teeth with generally similar size and morphology (Figure 38(A-C)). These teeth strongly resemble the teeth of some basal ornithischians (e.g. ankylosaurs) in having labiolingually flattened, triangular crown shape, mesiodistal carinae and well-developed cingulum basally on both sides. Apart from the presence of cingulum, these teeth are also reminiscent of the Late Triassic pterosaur Caviramus (Stecher 2008, Ösi 2011). Labiolingually, the tooth crowns are ornamented by fine, roughly parallel enamel ridges starting from the serrated carina down to the base of the tooth crown, as seen on the holotype tooth (Carvalho 1994:fig. 9). The serrations or cusples are not as large as in ankylosaurs and based on the mesial carina of the fifth right tooth, these carinae bear at least 8-12 cusples. Below the cingulum, at the junction of the tooth crown base and the root, the tooth is strongly constricted. Teeth are single rooted (Carvalho 1994).

The mandibular dentition is very poorly known. Based on the left anterior dentary fragment of UFRJ 113-R, it seems that the anterior 3-4 teeth in the dentary were conical, pointed and slightly labiolingually compressed. Their labial surface is smooth and no cingulum can be observed. A similar tooth morphology was described and illustrated by Carvalho (1994) in MN 4355-V and MN 4152-V (Carvalho 1994:fig. 5). If there was any carina, it was not well developed. The following teeth $\left(? 5^{\text {th }}\right.$ to $\left.? 7^{\text {th }}\right)$ are generally similar in morphology. They are triangular, pointed apically, their labial surface is smooth, and at least this side is devoid of cingulum. The posterior segment of the mandibular tooth row is unknown. However, at least five alveoli can be seen in the mandible of MN 4355-V (Carvalho 1994:fig. 3) that may indicate that the posterior mandibular teeth were closely packed, and probably similar in morphology to the upper, four cheek teeth. This hypothesis would be supported by the wear facets of the upper teeth. 
Regarding recent analogies of the teeth of Candidodon, the extreme heterodonty of this taxon prevents to find identical moprhology among extant reptiles. The non-procumbent premaxillary teeth are pointed and elongate with an apical angle lower than $45^{\circ}$. This strongly morphology resembles that of the teeth of insectivorous lizards (e.g. among teiids) in which teeth (or the main cusp of the tooth) are usually pointed to easily penetrate the hard exoskeleton of insects (Evans and Sanson 1998). On the other hand, apical angle increases in the anterior maxillary teeth $\left(60^{\circ}-80^{\circ}\right)$ and they already bear serrated carinae. The second maxillary tooth is quite similar to the lanceolate-shaped and cuspidate teeth of Iguana iguana which is a typical herbivorous form. The labiolingually flattened, enlarged, and most probably finely serrated caniniform with an apical angle close to $50^{\circ}$, however, again shows some affinities to insectivorous-carnivorous lizards. The posterior block of small, cuspidate teeth with cingulum and extensive tooth wear (see below), however, strongly differs from the anterior pointed teeth. They, again, show a number of similarities with the teeth of dominantly herbivorous forms (e.g. Cyclura, Ctenosaura). This diversity of the tooth morphology in the tooth row of Candidodon prevents the elucidation of the finer dietary habits of the animal.

\section{Dental wear}

As it is expected on the basis of complex tooth morphology, the teeth of Candidodon bear extensive wear facets (Figure 38(D-H)). The premaxillary teeth appear to be unworn with complete apical carinate regions. However, as it can be seen on the anterior maxillary and caniniform teeth, probably these fang-teeth bear also some fine wear on the enamel surface. On the second left maxillary and the following caniniform tooth, dental wear occurs apically and mostly along the carinae. These teeth are slightly worn as it is indicated by the smooth, shiny, and slightly rounded surfaces clearly observable, for example, on the apical region of 
the left caniniform. These worn surfaces, however, occur both labially and lingually, so they do not indicate direct occlusion of the upper and lower teeth in this anterior segment. The four post-caniniform maxillary teeth bear various developed wear facets on their lingual surface. These wear facets are usually steeply inclined (approximately $60^{\circ}-80^{\circ}$ relative to the horizontal plane) e.g. on the right $4^{\text {th }}$ and $5^{\text {th }}$ teeth but if the wear facet is more extensive as it is on the $6^{\text {th }}$ tooth (Figure $38(\mathrm{D})$ ), then this angle becomes lower (approximately $30^{\circ}-40^{\circ}$ ). Similar trend in the change of wear facets orientation can be seen in Notosuchus, Mariliasuchus and in sphagesaurids too. It is important to note that wear facets generally occur on the apex of the tooth crowns but in a more advanced state, the wear facets extend further along the serrated carinae and also onto the cingulum. Wear facets of the left and right sides are roughly similar but some differences can be also pointed out. The four teeth possess smaller, symmetrical, rhomboid-shaped wear facets (Figure 38(E)). On the left, one the dentine is exposed with marked step relationship of the EDI labially (Figure 38(G)). The dentine surface is dominated by irregular pits but some short scratches also occur. The orientation of these scratches is apicobasal or close to that. Here, obviously as a continuation of the apical wear facet, a steeply inclined, $1.5 \mathrm{~mm}$ long wear facet occur on the lingual cingulum but the dentine is not exposed. The $5^{\text {th }}$ tooth of the right side is almost complete, the dentine is not exposed and only some apical wear occurs but with informative wear pattern. It bears a high number of mesiodistally oriented, long (50-200 $\mu \mathrm{m})$ scratches, some apicobasally oriented, short scratches and irregular pits. On the left side, the $5^{\text {th }}$ tooth is newly erupted, thus being in a deeper position it does not show a marked wear pattern. The $6^{\text {th }}$ tooth is the most eroded on both sides. The apical wear facet is mesiodistally elongate but on the right side it also extends basally on the lingual surface of the crown. The wear facet of the left $6^{\text {th }}$ tooth is mainly on the mesial carina (Figure $38(\mathrm{~F}, \mathrm{H})$ ). Here, the EDI is mostly flush both labially and lingually but some rough, step relationship occurs at the mesial end of the wear 
facet. The dentine is exposed and slightly concave. Both the dentine and enamel bear few, roughly mesiodistally oriented short scratches. The $6^{\text {th }}$ tooth of the right maxilla is the most eroded among the teeth of this specimen (UFRJ-114-R). Here, the apical third of the crown is eroded and the dentine is exposed in a slightly concave surface. The wear facet can be divided into two parts. The apical part is longitudinal with an inclination of approximately of $30^{\circ}$ relative to the horizontal plane (Figure 38(C)). It bears mesiodistally oriented, long (50-300 $\mu \mathrm{m})$ scratches and some irregular pits. Most of its surrounding enamel margin is smooth, but labially, on its highest point on the crown, it is irregular and crushed. The second part of this wear facet is in connection with the first one but extends basally on the lingual surface of the crown. This surface is more steeply inclined, scratches are not characteristic on it, but it bears a high number of irregular pits. The $7^{\text {th }}$, last teeth are the smallest of the postcaniniform teeth and they do not show intensive wear on either side, only some weak apical wear can be observed.

Although the few teeth preserved in the left dentary of UFRJ-113-R are labially exposed, dental wear on them cannot be recognized because they are covered with conservation material.

\section{Adductor muscle reconstruction}

For the reconstruction of the jaw adductors of Candidodon, only the skull (UFRJ 114-R) can be used because the postdentary part of the mandible is unknown. Compared to Mariliasuchus or Sphagesaurus, the pterygoid-ectopterygoid complex of Candidodon is huge and steeply oriented ventrally. The lateral pterygoid flanges have an expanded posterior surface (Figure 37(E)) for the origin of MPTV which unambiguously indicates that this muscle was well developed and comparable to that of modern crocodylians (Busbey 1989). The suborbital fenestrae are narrow but strongly elongate relative to skull length. This indicates a great mass 
of MPTD originating from the posterior end of the nasal cavitiy and the margin of the surrounding bones of the suborbital fenestra. It can be concluded that the pterygoid muscles were the dominant adductors of great importance during jaw closure in Candidodon. In contrast to the pterygoid muscles, the external adductors appear to have been less developed. First, the skull was relatively narrow at the level of the temporal fenestrae. Second, the lateral temporal fenestra is much smaller than the orbits. Hence, the MAMES and MAMEM originating from the qudratojugal and the ventrolateral margin of the quadrates, respectively, were not so developed. Third, the anteroposteriorly elongate supratemporal fenestra is expanded, especially medially (Figure 37(A)), but its relative size is far from those of Mariliasuchus and sphagesaurids and it is more reminiscent to that of Malawisuchus. This indicates that MAMEP was not as much developed as in crocodyliforms using palinal movement. The laterosphenoid and the medioventral part of the supratemporal fossa are covered with sediment thus the origin surfaces of MPSS cannot be observed.

The ventral surface of the distal quadrate in Candidodon is rather smooth, only a single crest occurs close to the pterygoid-basioccipital contact. It is not clear if this crest corresponds to any of Iordansky's (1964) crests. This surface is the origin of MAMP which indicates that this muscle was not particularly developed but could have been an important muscle comparable to that of extant forms. Altogether, it can be concluded that among the jaw adductors of Candidodon, the pterygoid muscles were the dominant jaw closers and the external adductors were less developed, similarly to Malawisuchus and Notosuchus.

\section{Jaw mechanism and possible phases of chewing}

The smooth surface and the slightly eroded carinae on the caniniform, and the obviously complete crowns of the anterior teeth of Candidodon suggest that effective dental occlusion did not exist in the anterior portion of the tooth row, only some accidental occlusion of the 
upper and lower teeth occured. On the other hand, the obliquely oriented wear facets on the posterior cheek teeth clearly indicate a precise tooth-tooth contact in Candidodon at least in the back of the tooth row. Here, wear facet orientation and the determined orientation of scratches show a mainly orthal mandibular movement. This is further supported by the fact that in the early stage of wear, the central apical region of the teeth became eroded and later, this steeply inclined, apical wear extended on the mesiodistally positioned carinae. On the other hand, extended wear on the posterior teeth bear some mesiodistal scratches (Figure $38(\mathrm{H}))$. Being up to $300 \mu \mathrm{m}$ on $5^{\text {th }}-6^{\text {th }}$ teeth, these scratches are not as long as the scratches in the other notosuchians discussed earlier (up to 3-4 mm) but the teeth of Candidodon are also much smaller (length of the right $6^{\text {th }}$ tooth is $3 \mathrm{~mm}$ ). This may suggest that the mainly orthal jaw movement was complemented by some anteroposterior component. Some wear facets (e.g. on the right $6^{\text {th }}$ tooth) might also imply a longitudinal mandibular movement because the carinate edge of the tooth crowns are mesiodistally eroded so that the dentine is exposed in a long, slightly concave surface. These wear facets, however, could be also the result of simple orthal jaw closure, i.e. the obliqe carina of the opposing, lower tooth simply occluded with that of the upper one. Accepting the hypothesis that the process of jaw closure was accomplished by some anteroposterior movement, the determination of the exact direction of mandibular movement during powerstroke (i.e. proal or palinal) in Candidodon is not as simple as in earlier discussed crocodyliforms. In other forms with palinal movement, the obliquely oriented, long scratches unambiguously demonstrated the direction of mandibular movement during tooth-tooth contact. In Candidodon, however, most of the scratches on the mesiodistally elongate wear facets of the $5^{\text {th }}$ and $6^{\text {th }}$ teeth are parallel with the horizontal plane, their orientation does not have significant vertical component, so the scratches alone do not reveal whether the mandible moved backwards (palinal) or forwards (proal) in the powerstroke. Comparison of the $6^{\text {th }}$ maxillary teeth of the left and right sides shows an 
important difference concerning wear pattern. Whereas on the left side, the wear facet is on the mesial carina reaching the apex of the crown and the distal carina is almost complete, the right $6^{\text {th }}$ tooth possesses a more extensive wear facet in the whole apical region with most of the mesial and distal carinae eroded. This shows that on the left side the $6^{\text {th }}$ tooth is in an earlier stage of wear compared to the right side and that in this early stage of wear, the mesial and/or apical region of the teeth had become first eroded, at least on that tooth. This obliquely oriented wear on the mesial carina could have been produced by orthal movement when the apex and the distal carina of the complementary lower tooth occluded with this surface. An alternative option is that besides orthal movement, a backward sliding of the mandibles helped to bring these teeth into an antagonistic contact that could also have produced similar wear facets. This, however, was not necessary. The EDI on the right $6^{\text {th }}$ tooth further supports the latter, more complex version because it shows a more flush relationship mesiallymesiolabially, and a more irregular step relationship distally-linguodistally. Based on this information, a mainly orthal jaw closure with the possibility of some palinal component could be supposed. The reconstructed cranial adductor musculature not really supports this hypothesis. As it is known in extant crocodylians with exclusively isognathous, orthal jaw closure, the most important adductors are the pterygoid muscles (Iordansky 1964, Busbey 1989). Based on the adductor muscle reconstruction (see above), pterygoid muscles and MAMP were well developed and could have been the primary motors of the primarily orthal jaw closure also in Candidodon. In crocodyliforms with significant palinal movement, it was demonstrated above that the adductors responsible for pulling the mandibles backwards during the powerstroke must have been the external adductors because they have an anteroventral-posterodorsal orientation. Origin surfaces of these muscles in Candiodon, however, suggest that they were not particularly developed and perhaps demonstrate that anteroposterior shifting of the mandibles during the powerstroke was not significant. 
Due to the insufficient information on the wear pattern and the poorly preserved mandibles, assessing if dental occlusion was unilateral or bilateral is not possible. The symphyseal region is unknown, so it is also unclear whether it was fused or symphyseal mobility might have helped during dental occulsion. Nevertheless, the presence of a large caniniform, the generally similar wear pattern on the left and right sides of the upper teeth, and the wide lateral flanges of the pterygoids suggest that the closure of the mandibles of Candidodon was not anisognathus as in sphagesaurids and could not produce a lateromedial movement but instead, a precise, bilateral occlusion existed.

Based on dental wear and the reconstructed cranial adductors, it can be concluded that during powerstroke, the precise, mainly orthal closure of the jaws in the Early Cretaceous Candidodon may have included some anteroposterior movement of the mandibles (perhaps palinal) which, however, could not have been as significant as in the Late Cretaceous Mariliasuchus or in sphagesaurids.

Summarizing the available information, the following phases of oral food processing can be reconstructed:

1) Preparatory stroke starts with the opening of the mouth by contraction of MDM. This movement might be completed by the slight protraction of the mandibles motorized by the pterygoid muscles. It continues with the first contraction of adductor muscles to get the food item between the anterior teeth. In contrast to various earlier discussed forms, the anterior teeth of Candidodon are not procumbent, but the pointed, carinate and labiolingually slightly compressed teeth were able to grasp and hold the food item and take it into the mouth. The huge carinate caniniform in the maxilla with its slightly eroded, smooth enamel surface indicate that at the end of the preparatory stroke, this tooth was used to puncture the food item but it could also have been used to catch or slice the prey. In the prepapratory stroke, the main mandibular movement is simply orthal. 
2) When the food is in the mouth and its particles have been transported posteriorly to the cheek region, the powerstroke continues with the function of the four, labiolingually compressed posterior cheek teeth bearing tuberculate carinae and cingula. It is well known that more complex teeth in posterior part of the jaw reflect ,the greater bite force available further back in the jaw" (King 1996:111). In this phase, the trituration of the food is accomplished by the precise occlusion of these teeth. The first part of the powerstoke could have been dominated by simple orthal movement that produced the steeply inclined wear facets. Accepting the hypothesis of an additional anteroposterior movement of the mandibles in the powerstroke, it is unknown whether it characterized the end of every chewing cycle, as for example in Mariliasuchus or in gopher tortoises (Bramble 1974), or only a few chewing cycles performed by orthal closure were associated with some anteroposterior movement (perhaps palinal) during which the mesiodistally oriented scratches were being formed. Whichever is the case, it can be concluded that although an advanced oral food processing characterized Candidodon too, it was obviously not as developed as in its later, closely related relatives with palinal movement.

Notosuchia Gasparini, 1971

Pakasuchus kapilimai O’Connor, Sertich, Stevens, Roberts, Gottfried, Hieronymus, Jinnah, Ridgely, Ngasala et Temba, 2010

(Figure 39)

Holotype-RRBP 08631, articulated skull and skeleton.

Referred specimens - RRBP 05103, partial skull preserving left maxilla, lower jaw and eight postcaniniform teeth. 
Type Locality_Locality RRBP 2007-04, 20 km south of Lake Rukwa, Rukwa Rift Basin, Tanzania, Africa (see O’Connor et al. 2010, supplementary information).

Type Horizon—Red Sandstone Group, Galula Formation, Middle-Cretaceous. O’Connor and his colleagues (2010:2) noted that, the sequence is generally similar to that of the Dinosaur Beds of Malawi (the locality of Malawisuchus, Gomani 1997), located less than $150 \mathrm{~km}$ to the southeast of the fossiliferous portions of the Red Sandstone Group. Based on this and other unpublished data, the Galula Formation of the Red Sandstone Group is interpreted to represent the downstream equivalent to the Dinosaur Beds".

\section{General cranial and mandibular morphology}

Pakasuchus possesses a small $(6.1 \mathrm{~cm})$ and relatively narrow, oreinirostral skull that is comparable to that of Malawisuchus. The rostrum is short, approximately two-fifth of the total skull length and slightly constricted just in front of the orbits. The orbits are large, comparable in size with that of Malawisuchus but not as large as in Mariliasuchus, Adamantinasuchus or Yacarerani, and they are facing anterolaterally. Although the premaxillae and nasals are poorly (or not) preserved in both specimens, an anterior direction of the external nares was predicted for Pakasuchus (O'Connor et al. 2010), similarly to other notosuchians. The supratemporal fenestrae are anteroposteriorly elongated (almost four times longer than lateromedially wide) and together with the supratemporal fossa they are relatively small (Figure 39(A)), similarly to Malawisuchus and Notosuchus. Unfortunately, the fenestrae are completely filled with matrix so the extension of the shelf of the fenestrae is not discernable. The lateral temporal fenestrae are triangular-shaped and moderate in size (Figure 39(B)). Behind the bony secondary palate, the choanae are bordered by the pterygoids that ,have a rostrolaterally [and ventrally] directed flange with a distinct ventrally directed caudal margin" (O’Connor et al. 2010, suppl. information, p. 6). This feature, used by these authors as a 
diagnostic character of Pakasuchus is really strange, but a roughly similar orientation of the pterygoid flanges can be seen for example in Simosuchus. In addition, Mariliasuchus also shows a similar orientation of the pterygoid flanges, although not as strongly anteriorly oriented as in the former taxa. On the other hand, the size and orientation of the pterygoid of Pakasuchus strongly differs from the laterally extended and posteriorly oriented pterygoids of Malawisuchus. The distal end of the short quadrates has a ventral-posteroventral orientation with a deep and wide groove on the articular end cleraly separating the medial and lateral condyles. The medial condyle is projected more ventrally than the lateral one. Although a similar morphology of the quadrate condyles can be seen in various crocodyliforms, a strongly articulating divided, bi-planar surface of the glenoid surface can be observed only in Pakasuchus and Simosuchus (Kley et al. 2010). On the articular, the glenoid surface is two times longer than the ventral surface of the quadrate condyles and it is divided into a dorsally facing, lateral facet and a dorsomedially facing medial facet that is close to vertical (approximately $70^{\circ}$ relative to the horizontal plane; this medial surface in Simosuchus is approximately $45^{\circ}$ relative to the horizontal plane). This results in a precise quadrate-articular joint with the longitudinal ridge of the articular fitting into the intercondylar groove of the quadrate. The precise articulation is further supported by the high lateral wall of the surangular. The jaw joint is at the level of the occlusal plane. The temporal cavity is still covered with matrix so the inner morphology of the surrounding bones including the origin surfaces of the adductor muscles are unknown.

The mandible is robust and its postdentary part is high, and slightly longer than the dentary (Figure 39(B)). It possesses a huge, oval external mandibular fenestra that is bordered dorsally by a dorsoventrally high and strongly elongate surangular. The mandible behind the fenestra is still massive, anteroposteriorly elongate, and its outer surface is smooth for the insertion of the ventral pterygoid muscles. The mandibular symphysis is fused. 


\section{Dentition and possible extant analogues}

Among the heterodont crocodyliforms, definitely Pakasuchus has the most mammal-like dentition (O'Connor et al. 2010) that is supported by the extreme, mammal-like regionalization (caniniform, premolariform, and molariform teeth), the extreme reduction in the number (five) of the upper teeth, and the complex, complementary surfaces of the upper and lower molariform teeth. The teeth anterior to the upper caniniform tooth (i.e. premaxillary teeth) are unknown in Pakasuchus and no anterior teeth are present anterior to the lower caniniform either. The enlarged upper caniniform (crown length ca. $7 \mathrm{~mm}$ ) is conical, distally curved and does not bear any enamel wrinkles or carina. The lower caniniform is similar in morphology to the upper one but it is sligthly smaller (crown length ca. 5-6 mm) and more anteriorly positioned. Although the premaxilla is not preserved, based on the articulated jaws of the type specimen (RRBP 08631) it seems that the lower caniniform fits into a socket or in an extended groove present between the maxilla and the premaxillary region. The teeth in the upper tooth row are relatively widely spaced (1.5-2 $\mathrm{mm}$ space between successive teeth) and the upper caniniform is followed only by a single small (crown length ca. $2 \mathrm{~mm}$ ), labiolingually slightly compressed conical tooth. In contrast to the single premolariform tooth in the upper jaw, the dentary includes five, closely-spaced premolariform teeth behind the caniniform. These conical and labiolingually slightly compressed teeth are similar in size (crown length ca. 1.5-2 $\mathrm{mm}$ ).

The single upper premolariform tooth is followed by three molariform teeth among which the anterior two $\left(3^{\text {rd }}\right.$ and $4^{\text {th }}$ in the maxilla) are the largest (mesiodistal crown length of the $3^{\text {rd }}$ is 5 $\mathrm{mm}$ and that of the $4^{\text {th }}$ is $4 \mathrm{~mm}$ ). These anterior two upper molariform teeth are mesiodistally elongate and have a similar morphology. Their occlusal surface is divided by a relatively wide and deep trough that has a mesiodistal or slightly labiomesial-linguodistal orientation. This 
trough is deeper distally, so this occlusal surface is facing distoventrally. The trough is bordered by a labial triangular crest with a rather distally positioned apex and by a lingual crest with a mesially and in unworn teeth probably with a distally positioned cusp. The third upper molariform tooth is similar in morphology to the two anterior ones but it is only $2 \mathrm{~mm}$ in length. The dentary contains only two molariform teeth that are the complementary elements of the two upper anterior molariform teeth. They also possess a deep and wide trough with a mesiodistal or slightly labiomesial-linguodistal orientation but the trough is deeper mesially so its occlusal surface is facing mesioventrally (as a complementary occlusal surface of the upper crest). The lingual, triangular crest is positioned mesially with a low apex on its distal end. The more robust labial crest is extended along the whole crown being higher distally with a high apex at the distal end. O'Connor et al. (2010) reported on the replacement teeth of Pakasuchus that shows a similar morphology to that of the functional ones.

The reduction of tooth number, the characteristic regionalisation and tooth morphology in the dentition of Pakasuchus indicate that extant analogues can be found among mammals rather than reptiles. Indeed, among extant reptiles possessing teeth (lepidosaurs, crocodiles, tuatara), this kind of regionalisation of the dentition with so precisely fitting molariform teeth are unknown. Teeth of Pakasuchus resemble in some aspects the teeth of carnivorous mammals. The large caniniform teeth are designed for tearing, while the molariform teeth have longitudinally oriented, relatively sharp edges for cutting. These sharp edges on the molariform teeth (i.e. the labial crest on the lower molariform tooth and the medial crest on the upper one) are similar in some aspects to the carnassial teeth of some carnivore mammals. Their function, however, was not completely the same because mammalian carnassial teeth $\left(\mathrm{P}^{4}-\mathrm{M}_{1}\right)$ precisely meet and become abraded along a steeply inclined, almost vertical surface (the lingual surface of the $\mathrm{P}^{4}$ and the labial surface of $\mathrm{M}_{1}$ ). In Pakasuchus, the crests on the molariform teeth fit into a deep and wide trough and they become lower and blunt during the 
process of dental wear (see fig. S3 of O' Connor et al. 2010, suppl. information) and it seems that their shearing mechanism rather turned into a crushing and grinding function (see below).

\section{Dental wear}

Due to the preservation and articulated jaws of the specimens of Pakasuchus, only little direct information is available on the wear pattern of the teeth (P. O'Connor 2011, personal communication). Figure S3 of O' Connor et al. (2010) shows important difference in the molariform tooth morphology between erupted and unerupted teeth, namely that the labial crest of the second lower, erupted molariform tooth is much lower and not really crest like compared to that of the unerupted one. These authors interpreted this feature as the result of abrasion caused by its occlusion with the lingual crest of the upper molariform tooth. Indeed, studying the other molariform teeth on the video files of the supplementary information of O'Connor et al. (2010), similar eroded crest can be also seen on other teeth. The detailed morphology of these extensive wear facets (i.e. scratches, pits, EDI) is, however, still unknown.

\section{Adductor muscle reconstruction}

Most of the origin and insertion surfaces of cranial adductor muscles are still covered with matrix in Pakasuchus, thus the mass and proportion of jaw adductors can be only roughly estimated. The pterygoid-ectopterygoid morphology of Pakasuchus is different from that of Malawisuchus. O'Connor et al. (2010) noted an anterolateroventral orientation of the pterygoid flanges which possess a distinct, ventrally oriented caudal margin. Although this distinct, ventrally oriented caudal margin might reflect extended origin surfaces of MPTV, the relative size of the pterygoid flanges is not as large as in Malawisuchus or in extant forms. This indicates a less developed MPTV compared to that of Malawisuchus. On the other hand, 
in modern forms, MPTV has insertion surfaces on the posterolateral part of the mandible wraping around the retroarticular process to attach to the usually smooth, caudolateral surface of the angular (Holliday and Witmer 2007). This surface is fairly extended in Pakasuchus both anteroposteriorly and dorsoventrally (O’Connor et al. 2010: fig. 1b) suggesting a relatively well developed MPTV. The inner morphology of the mandibular adductor fossa is mostly unknown, so the insertion surfaces of MPTD and MAMP cannot be detected. This is also the case with the ventral surface of the quadrate-qudratojugal-basicranium complex, so the origin surfaces of MAMES, MAMEM and MAMP cannot be studied. Only the strongly elongate and massive surangular indicates an extended insertion surface for MAMES and MAMEM which might have been well developed. With their anteroposteriorly elongate shape and small size, the supratemporal fenestrae of Pakasuchus are more similar to those of Malawisuchus, Notosuchus and Candidodon than to those of crocodyliforms characterized by palinal movement (i.e. Mariliasuchus, sphagesaurids), so the MAMEP originating from this area could not have been an important muscle in Pakasuchus (Figure 39(A)). The laterosphenoid and the medioventral part of the supratemporal fossa are covered with sediment thus the origin surfaces of MPSS cannot be observed. To sum up, the jaw adductors of Pakasuchus appear to have been rather similar to Malawisuchus despite that the pterygoid morphology and the orientation of the pterygoid flange is different in the two taxa. The relatively narrow temporal region of the skull with small to moderate sized supratemporal fenestrae suggests less developed external adductors.

\section{Jaw mechanism and possible phases of chewing}

Pakasuchus possesses the most regionalized and complex dentition among heterodont crocodyliforms that is more similar to that of mammals than to any other crocodyliforms. The strongly reduced dentition includes two pairs of bi-crested molariform teeth which ensured a 
precise, interdigitating occlusion: ,whereas the lateral crest of the lower tooth slots into the trough of the upper molariform, the medial crest of the upper tooth occupies the trough of the lower molariform" (O’Connor et al. 2010, suppl. information, p. 6). The anteroposteriorly elongate and bi-plan glenoid surface allowed an anteroposterior sliding of the mandible, but it is still questionable whether there was any kind of anteroposterior movement during the powerstroke and if yes, whether this movement was proal or palinal. Because no details on the wear facets are available to inform us on the direction of mandibular movement, it cannot be unambiguously asserted that besides precise orthal jaw closure any kind of anteroposterior shifting of the mandibles really existed; a situation that is quite similar to that of Notosuchus. The only available tools for the reconstruction of the possible jaw mechanism are the tooth morphology and the rough estimation of the mass of adductor muscles. The approximately longitudinally directed trough on both the upper and lower molariform teeth has a slightly oblique, mesioventral-dorsodistal orientation, so the lower trough faces slighly mesially and the upper one faces slightly distally. The largest surface of these teeth participating in the direct occlusion of the crowns are the labial surface of the lingual crest on the upper teeth and the lingual surface of the labial crest on the lower teeth. Similarly to the trough, these surfaces also have an oblique, mesioventral-dorsodistal orientation (see O'Connor et al. 2010, supplementary video files). Thus, when these crests of the upper and lower molariform teeth occluded with each other, the easiest way to move the lower jaw and produce the most efficient shearing bite by these surfaces is a combination of backward and slightly upward movement of the mandibles. In this case, when the upper and lower teeth were in an occluded position and the fused mandibles started to shift posteriorly, the lower jaws came slightly closer to the upper ones. This scenario appears to be a much safer mode of handling the food item than the proal mandibular movement would be during oral food processing. This means that, similarly to the South American Mariliasuchus, Sphagesaurus, Armadillosuchus, and 
Yacarerani, Pakasuchus may have had an orthal jaw closure complemented with a retractive powerstoke (palinal movement). However, reconstructed adductor musculature does not really support this hypothesis because it seems that external adductors, generally being strongly developed in crocodyliforms with palinal movement, were not so much pronounced, powerful muscles in Pakasuchus. This suggests that if orthal jaw closure was really complemented by the retraction of the mandibles, then this posterior movement could not have been as significant as in Mariliasuchus and sphagesaurids. The lower caniniform most probably fitted into a hole or notch between the premaxilla and maxilla thereby preventing considerable longitudinal (and also transverse) movement of the mandibles. This is further supported by the fact that in forms characterized by proal, palinal or transverse movement, the lower caniniform is always missing. Similar enlarged lower caniniforms also occur in the protosuchian Edentosuchus and the Kayenta form. The presumed notch of the caniniform is, however, not preserved in Pakasuchus, thus the size of this cavity along with the degree of the possible backward movement of the mandibles during the powerstoke is not to be assessed. An alternative hypothesis for the anteroposterior movement in the jaw mechanism of Pakasuchus would be the forward shifting of the mandibles in the last phase of jaw closure (i.e. proal movement), similarly to that reconstructed in Malawisuchus (Clark et al. 1989). The roughly longitudinally oriented troughs on the molariform teeth, the relatively developed pterygoid muscles and the less advanced external adductors support this hypothesis. However, in this case, if the lower teeth had slide anteriorly on the slightly obliquely, mesially sloping troughs, the mandibular rami would have been forced to diverge from the upper jaw exactly in the chewing phase which would not have been desired during oral food processing when the food item must be held in the mouth. Thus, if there was any anteroposterior component of the mandibular movement during jaw closure in Pakasuchus, it would have rather been palinal than proal. Based on these uncertainties, however, in this work I prefer the reconstruction of a 
simple orthal jaw closure with a precise tooth-tooth contact in Pakasuchus with the note that an anteroposterior (possibly palinal) movement might have complemented the orthal jaw closure.

Description of the different phases of jaw mechanism in Pakasuchus is quite difficult in the light of uncertainities related to the presence or absence and direction of anteroposterior mandibular movement during the powerstroke.

\section{Heterodont notosuchians with orthal jaw movement}

Among the diverse notosuchians a great number of forms possessed some kind of functional heterodonty. In various species with extreme heterodonty and complex dentition (taxa that were discussed above), a precise dental occusion associated with a specialized jaw mechanism can be reconstructed. Nevertheless, there are at least two notosuchian taxa (Simosuchus [Kley et al. 2010] and Uruguaysuchus [Rusconi 1933, Soto et al. 2011]) which show a marked heterodonty sometimes with highly complex tooth morphology but apparently neither dental occlusion nor a complex mandibular movement characterizes them. In spite of that, these forms will be discussed shortly in this chapter because their highly complex tooth morphology along with some other cranial features indicate that intraoral food processing (i.e. tooth-foodtooth contact) could have had a significant role during feeding.

An additional species that is usually regarded as being closely related to heterodont notosuchians (Turner and Sertich 2010) is Libycosuchus brevirostris (Figure 40). Libycosuchus (Stromer 1914) has a small, massive, short-snouted and relatively high skull being quite similar to that of heterodont notosuchians (BSM 1917 VIII 574a, Buffetaut 1976, 1982). Although its dentition is almost completely missing or damaged, the high number of alveoli (over 17 in one side of the upper jaw, Figure 40(B)) and the posteriorly decreasing size of alveoli do not suggest a unique or particularly complex dentition. Buffetaut (1976) 
supposed a dentition similar to that of Theriosuchus with pointed, conical teeth anteriorly and blunter, labiolingually flattened and perhaps serrated teeth posteriorly. This hypothesis is further supported by the mesiodistally elongate posterior alveoli. The highly positioned quadrate-articular joint is quite similar to that of extant crocodylians, although the distal quadrate condyles are more ventrally oriented. The glenoid surface on the articular is not longer anteroposteriorly than the anteroposterior width of the distal condyles of the quadrate and the glenoid surface is bordered posteriorly by a transverse buttress and laterally by the dorsal margin of the surangular, similarly to extant crocodylians (Iordansky 1973). This unambiguously demonstrates a precise quadrate articulation and the absence of any anteroposterior or lateromedial mandibular movement during jaw closure.

Besides notosuchians various other mesoeucrocodylian taxa, for example, Araripesuchus spp. (Ortega et al. 2000, Pol and Apesteguía 2005, Turner 2006, Sereno and Larsson 2009), peirosaurids (Price 1955, Gasparini et al. 1991, Carvalho et al. 2007), the enigmatic Lusitanisuchus (Schwarz and Fechner 2004), atoposaurids (Owen 1879, Schwarz and Salisbury 2005, Martin et al. 2010), ziphodont forms (e.g. Buffetaut 1979, Company et al. 2005, Ösi et al. in press) possess heterodont dentition composed of complex teeth that usually occur in the posterior segment of the tooth row. Well-controlled dental occlusion and specialized (not simply orthal) jaw mechanism, however, most probably did not exist in these forms. Similar to Libycosuchus, the quadrate of these forms precisely fits into the glenoid surface of the articular and their teeth do not bear significant wear. If there is any wear pattern (e.g. on the distal part of the teeth of Araripesuchus patagonicus [Ortega et al. 2000]), then it is related to tooth-food-tooth contact or in some cases to accidental occlusion of the upper and lower teeth. Regarding dental occlusion, the only exception is Araripesuchus wegeneri where the labiolingually flattened, apically rounded posterior teeth bear steeply inclined wear facets that are certainly the result of precise tooth-tooth contact (Sereno and Larsson 2009, see 
also below). Similar to Libycosuchus, the precise jaw joint certainly prevented any anteroposterior or transverse movement of the mandibles. Jaw closure in these mesoeucrocodylians was simply orthal, and the complex dentition rather reflects some degree of adaptation to particular food resources and/or habitats rather than increasing the efficiency of oral food processing via dental occlusion.

\section{Systematic Palaeontology}

Mesoeucrocodylia Whetstone and Whybrow, 1983

Notosuchia Gasparini, 1971

Simosuchus clarki Buckley, Brochu, Krause and Pol, 2000

(Figure 41, 42)

Holotype-UA 8679, complete skull, and articulated partial postcranial remains (Buckley et al. 2000).

Referred specimens-Numerous specimens have been referred to this species that are listed in in the recent work of Krause et al. (2010). Among these, FMNH PR 2596 and FMNH PR 2597 show well preserved cranial remains (Kley et al. 2010).

Type Locality_Berivotra Study Area, Mahajanga Basin, northwestern Madagascar (Krause et al. 2010).

Type Horizon-Anembalemba Member, Maevarano Formation, Late Cretaceous, Maastrichtian.

Additional localities and horizons_-Altogether, 18 localities are known. Besides the Berivotra Study Area, specimens are known from the Masiakakoho Study Area, Mahajanga Basin, 
northwestern Madagascar. The Masorobe Member of the Maevarano Formation also contained remains of Simosuchus (Krause et al. 2010).

Remarks_-Simosuchus clarki was originally described by Buckley and colleagues in 2000. In 2010, in a monumental work edited by D. Krause and N. Kley, a very detailed description of S. clarki was published including the complete skeletal anatomy, taxonomy, and phylogeny of this bizarre crocodyliform. In addition, the paleoecological, paleobiogeographical and some paleobiological aspects of the animal were also discussed. Regarding the detailed craniodental features of the animal, the description of Kley et al. (2010) is certainly the reference work, thus in the following sections I strongly focus only those craniodental features that are related to the feeding of Simosuchus.

\section{General cranial and mandibular morphology}

Simosuchus possesses an extremely short (total skull length between $115 \mathrm{~mm}$ [FMNH PR 2597] and 128 mm [UA 8679]), box-like, platyrostral skull (sensu Busbey 1995) with very short, two times wider than long, rectangular rostrum (Figure 41(A)). The laterally facing orbits are huge being almost two times larger than the supratemporal fenestrae. The triangular laterotemporal fenestrae are strongly reduced being almost six times smaller than the supratemporal fenestrae. The lateromedially wide and divided external nares are anteriorly oriented. Supratemporal fenestrae are relatively large and slightly trapezoid occupying approximately $50 \%$ of the total area of the skull roof. The supratemporal fenestra is approximately three times more extended than the supratemporal fossa. The distal end of the quadrate has a ventral and slightly anteroventral orientation which is quite unusual among crocodyliforms (Buckley et al. 2000). The bony palate is extremely wide and emarginated by the rows of the relatively small alveoli. The lateral process of the pterygoid has an anterolateroventral orientation and borders the relatively large suborbital fenestra. This lateral 
process has a ventrally oriented caudal crest. Laterally, the process has a massive, stocky end which is fused anteriorly with the very short, twisted and vertically oriented ectopterygoid. The anteriorly projecting median process of the pterygoid separates the huge, anteroposteriorly elongate (ca. $2 \mathrm{~cm}$ long) choanae medially (Figure 41(B, C)). Along with the anteroventrally oriented quadrates, the basioccipital has a great anterior expansion, so that the temporal cavity is also in a more anterior position compared to other crocodyliforms. The jugal is a quite massive and lateromedially wide element bordering partly the lateral temporal fenestra. Posteriorly, it connects to the quadratojugal which is a very short and lateromedially wide bone. The occipital condyle is posteroventrally oriented (Kley et al. 2010).

The short and massive mandible of Simosuchus is relatively high compared to its length (Figure 41(E)). The tooth row is almost at the level of the dorsalmost part of the postdentary part. The two hemimandibles are strongly fused via a relatively short but dorsoventrally high symphysis. Due to the wide and rectangular rostum, the dentaries also show a rectangular construction, thus they have a lateromedially extended, anterior part that contains the half of the alveolar groove ( 7 from the 14 teeth). The angular bears an ornamented, rugose ventrolateral flange that is very similar to those seen on the mandibles of ankylosaurs (Vickaryous et al. 2004).

As it was emphasized by Kley et al. (2010), the quadrate-mandible articulation is positioned strongly below the level of the occlusal plane (Figure 41(C, E)). Posteriorly, the articular together with the posterior end of the angular project posteroventrally and the ventral surface of the angular in this region is smooth and strongly concave (the bundles of MPTV wrapped along this surface, Holliday and Witmer 2007). The retroarticular process is short and projects posteroventrally. The glenoid surface is relatively shorter than in most previously mentioned, heterodont notosuchians (Figure 41(D)) but as it was noted by Kley et al. (2010:) it is „,nearly twice the length of the condyles of the corresponding quadrate". Furthermore and in contrast 
to extant crocodylians, no transverse buttress is present behind the glenoid surface to prevent posterior sliding of the mandibles. Due to the posteroventral inclination of the whole posterior end of the mandible, the glenoid surface is facing dorsally and slightly posterodorsally (see Kley et al. 2010:fig. 25A). Similarly to Pakasuchus, the glenoid surface is divided into a lateral facet and a dorsomedially facing medial facet the latter of which slants approximately $45^{\circ}$ relative to the horizontal plane. As in all crocodyliforms, laterally the glenoid surface is bordered by the elevated surangular to prevent lateromedial translation of the mandibles. A significant part of the tooth row is in the anterior, transversely oriented part of the dentary. The postdentary part of the mandible is relatively long. The surangular is wide and massive, and it is ornamented dorsally by some neurovascular foramina (Kley et al. 2010).

\section{Dentition and possible extant analogues}

Kley et al. (2010) gave a detailed description of the teeth of Simosuchus, and provided a comparison of its teeth with a variety of those extant and extinct reptiles that possess similar, labiolingually compressed, multicusped teeth. Thus, here I mention only the most important dental characters of Simosuchus from which functional inferences can be drawn related to feeding mechanism. Simosuchus bears five premaxillary, 11 maxillary and 14 dentary teeth in one side of the skull. Tooth crowns are mesiodistally elongate and they do not bear a cingulum but there is a strong constriction between the root and the crown on each tooth (Figure $41(\mathrm{~F}, \mathrm{G})$ ). The tooth surface is smooth or very slightly ornamented by some shallow, longitudinal enamel wrinkles. Premaxillary teeth are tripartite composed of one central, one mesial and one distal „prong” (Kley et al. 2010:77). The central prong ends in three cusps and the side prongs end in two small, pointed cusps. A deep sulcus is present between the prongs (Figure 41(G)). Based on Kley et al. (2010: fig. 30), the anterior four maxillary teeth are quite similar to those of the premaxillary teeth but they are slightly more elongate mesiodistally. 
Posteriorly, the cuspidate crowns tend be lower and the tripartite morphology rather transforms into a single block composed of roughly similarly sized cusps among which the marginal cusps are always the smallest. Dentary teeth are generally similar to those of the upper tooth row. The anterior four or five dentary teeth are similar to the premaxillary and anterior maxillary teeth in having a well distinguished central prong and accessory mesial and distal prongs that are ornamented by cusps (three in the central prong and two-two in the accessory prongs). From the fifth dentary tooth, the crown of the teeth becomes lower and mesiodistally relatively more elongate. The prongs are not so well distinguished from each other. The cusps, however, are more uniform and well separated from each other similarly to those of the upper tooth row.

In the anterior segment of the upper tooth row (premaxillary and first and second maxillary teeth), teeth have a ventral or posteroventral orientation. The anteriorly positioned dentary teeth have a dorsal or anterodorsal orientation. Replacement of the teeth has been reported in Simosuchus and it does not seem to show any organized pattern (Kley et al. 2010). Of the five premaxillary tooth positions, three contain replacement teeth on each side. In the case of the 11 maxillary teeth, however, only one tooth (second) on the left side and two teeth (second and third) on the right side bear replacement teeth in their root. In the case of the dentary, five teeth reveal replacement teeth, among which the posteriormost tooth is the $10^{\text {th }}$. So it seems that tooth replacement rate of the anterior teeth was higher.

As noted by Buckley et al. (2000) the teeth of Simosuchus show a great similarity with the teeth of ankylosaurs and stegosaurs, which have long been thought to have been dedicated herbivores. Kley et al. (2010:90) demonstrated great similarity between the teeth of Simosuchus, extant herbivorous lizards and other taxa, and based on the works of Buckley et al. (2000), Galton (1986) and Reisz and Sues (2000), they collected five characters that strongly correlated with herbivory among other vertebrates including extant forms. These are 
(1) the labiolingual compression and marked mesiodistal expansion of the teeth; (2) the

presence of numerous distinct cusps along the mesiodistally oriented apical region; (3) crowns are spatulate and has a shallow lingual concavity near its base; (4) dentition is closely packed with little or no gap intervening between them; (5) the crowns are oriented in an 'en echelon' arrangement. All of these dental characters are present in Simosuchus and clearly suggest a predominantly herbivorous diet.

\section{Dental wear}

Some cusps on the teeth of UA 8679 are worn but these abraded surfaces are not the result of tooth-tooth occlusion (Kley et al. 2010). Only the lingual surface of the first right and second left premaxillary teeth show some wear facets that could have been produced by tooth-tooth occlusion (Figure 41(G)). No more details (scratches, pits, EDI) of the wear facets are known.

\section{Adductor muscle reconstruction}

The skull and mandible of Simosuchus show a great number of specializations that strongly reflect the size and position of the origin and insertion surfaces and the orientation of jaw adductors (Figure 42). The anterolateroventrally oriented and reduced, rod-like, lateral pterygoid processes indicate that MPTV was a significantly reduced muscle compared to extant forms. Originating from the posterior part of the pterygoid flanges, the bundles of MPTV pass posteriorly and slightly posterolaterally and wrap around the retroarticular process to attach to the smooth caudolateral surface of the angular (Holliday and Witmer 2007). The deep position of this attachment surface on the angular of Simosuchus results in a slight ventral component in the orientation of MPTV and thus decreases the moment arm of this muscle (Kley et al. 2010). On the other hand, the region around the suborbital fenestra, the origin surface of MPTD, is well extended and is in a much more dorsal position than that 
of MPTV. Furthermore, the anterior shifting of the pivot point (by the anteroventral orientation of the quadrate) and thus that of the insertion surface of MPTD in the mandibular adductor fossa indicate a shortening and reorientation of this muscle, namely in a more vertical position. These features suggest a short and relatively developed MPTD in Simosuchus (Figure 42(A)).

The unusual anteroventral orientation of the quadrate together with the deeply positioned craniomandibular joint (Kley et al. 2010) resulted in important changes in the orientation and probably power of MAMP, MAMES and MAMEM. With the anteroventral orientation of the quadrate, its anteroventral surface and also that of the quadratojugal are close to vertical and not anterodorsal-posteroventrally oriented as in modern crocodylians, so that their origin surfaces are almost dorsal to the pivot point (Figure 42(A)). However, due to the deeply ventrally positioned pivot point, the insertion surfaces of MAMEM and MAMES on the surangular are in a more dorsal postition, thus it shortens the distance between the origin and insertion points of these muscles and increases their moment arm (Kley et al. 2010). Regarding the origin surfaces of MAMP, MAMES and MAMEM, no crests discussed by Iordansky (1973) in extant forms or seen in other heterodont notosuchians, or protuberances can be observed. The features observed in Simosuchus refer to powerful but not particularly developed muscles in this region.

The supratemporal shelf is strongly extended posteriorly and medially (Figure 42(B)) indicating a well developed MAMEP originating from here, similarly to heterodont notosuchians with palinal movement. The efficieny of MAMEP was further enhanced by the deeply positioned craniomandibular joint resulting in a higher attachment area of the posterodorsally-anteroventrally oriented adductors (i.e. MAMEP, Kley et al. 2010). Simosuchus possesses a well-developed cotylar crest that separates the extended anterolateral and posterolateral laminae of the laterosphenoid (Kley et al. 2010). On the ventral half of the 
cotylar crest a developed tensor crest is present just dorsal to the laterosphenoid contact with the epipterygoid. However, Kley et al. (2010:53) noted that ,the laterosphenoid of Simosuchus lacks a distinct impression or crest ('longitudinal oblique crest' of Iordansky [1964] or 'cotylar crest' of Busbey [1989]), corresponding to the attachment site for a muscle identified by Iordansky (1964) and Busbey (1989) as the M. adductor mandibulae externus profundus, but more recently by Holliday (2009) as the M. pseudotemporalis superficialis". This indicates the MPSS was probably not a really developed cranial adductor in this form. To sum up, the anteriorly shifted and deeply positioned craniomandibular joint, the overall reduction in the length of the lower jaw, and the relatively powerful external adductors resulted in an increase in the mechanical advantage of the mandibles of Simosuchus (Kley et al. 2010). The reduction of MPTV is, however, also well demonstrated, so I agree with the conclusion of Kley et al. (2010:91) that "Simosuchus was likely not able to bite with particularly great force". This is further supported by the anteroposteriorly open glenoid fossa that was most probably fixed in an articulated position only by the cranial adductors.

\section{Jaw mechanism and possible phases of chewing}

Although Simosuchus can be characterized by a highly complex jaw apparatus, a great number of cranial and mandibular modifications compared to a traditional crocodyliform skull, and by complex, multicusped teeth, a well-controlled tooth-tooth occlusion was not present in this peculiar animal. Extensive wear being as a possible evidence for direct toothtooth contact has been reported only in the case of the first right and second left premaxillary teeth of the type specimen (Kley et al. 2010). Study of many other heterodont crocodyliforms characterized by heavy dental wear and occlusion shows, however, that if well-controlled and regular dental occlusion occured in any of the taxa, a high percent of the cuspidate (generally posterior) teeth bear developed wear facets. Because most of the teeth of Simosuchus are 
devoid of any significant wear, the anterior facets on the premaxillary teeth (Figure $41(\mathrm{G})$ ) rather appear to be the result of some accidental occlusion that might have been caused by the different (slightly oblique) orientation of anterior teeth.

Buckley et al. (2000) described that the glenoid surface of the articular is not as long anteroposteriorly as in other notosuchians and so they suggest a limited anteroposterior movement of the mandibles in Simosuchus. On the other hand, Kley et al. (2010) concluded that the glenoid surface having twice the anteroposterior length of the quadrate condyles indeed could have ensured a limited anteroposterior movement of the lower jaws. However, in extinct (e.g. dicynodonts, allotherian mammals, other notosuchian crocodyliforms [King 1996], diplodocid sauropods [Upchurch and Barrett 2000]) or extant (e.g. tuatara, rodents, kangoroos) animals characterized by proal or palinal movement, an efficient dental occlusion with developed wear facets on the teeth always occurs that was apparently not present in Simosuchus. So I suggest that in spite of the anteroposteriorly elongate glenoid surface, the jaw closure in Simosuchus was simply orthal with relatively simple shearing bite (but without tooth-tooth contact) roughly similar to some extant herbivorous lizards possessing similar, labiolingually compressed, multicusped teeth (for comparison of the teeth see Kley et al. 2010:fig. 37). Kley et al. (2010) discussed the mechanical consequences of the deeply ventrally positioned glenoid surface which, in contrast to most other heterodont notosuchians, is well below the level of the tooth row. They found that this feature increased the moment arm of the external mandibular adductors (e.g. MAMES, MAMEM, MPS), decreased the moment arm of the pterygoid muscles, and „resulted in the entire lower tooth row approaching the entire upper tooth row at only a very shallow angle during jaw closing, thereby maximizing the number of teeth that would have simultaneously contacted a given food item contained either partially or fully within the oral cavity" (Kley et al. 2010:92). 
The very broad and rectangular rostrum of Simosuchus (Figure 41(B)) could have been an additional adaptation to maximize the capacity and efficiency of the feeding apparatus. Roughly similar, rectangular oral cavity appeared, for example, in several herbivorous sauropod dinosaurs (e.g. Diplodocus Holland 1924, Bonitasaura Apesteguía 2004, and the most extreme example is Nigersaurus Sereno et al. 1999, 2007). Among these, nemegtosaurids, which also have a rectangular rostrum, possess extensive tooth wear (at least Nemegtosaurus, Nowinski 1971) and a slightly elongated glenoid surface the latter of which was interpreted as indicating the possibility of a moderate amount of anteroposterior mandibular movement during jaw closure (Upchurch and Barrett 2000). Nevertheless, Upchurch and Barrett (2000:103) also noted that the role of this jaw movement is not completely clear, ,although it may have widened the gape or facilitated correct relative positioning of the upper and lower tooth rows during the bite phase". If Simosuchus was indeed characterized by a proal or palinal movement of the mandibles, then this movement was less important than in other notosuchians with heterodont dentition and tooth wear, and could have had a similar role to that supposed in nemegtosaurid sauropods but certainly not to provide well-controlled tooth-tooth contact (i.e. chewing).

Notosuchia Gasparini, 1971

Uruguaysuchus aznarezi Rusconi, 1933

Holotype-CA, unnumbered.

Referred specimens_-Originally 7 specimens of Uruguaysuchus has been found. One of these represents the holotype of $U$. aznarezi and another one repesents the holotype of $U$. terrai. The additional five specimens have been lost. Newly discovered materials are: FC-DPV 2320 (partial skull, mandible and some elements of the axial skeleton), MPMAB 2881 (two post- 
caniniform teeth, one osteoderm and a partial humerus) and MUNHINA 451 (two fragmentary vertebrae) (Soto et al. 2011).

Type Locality_ Guichón, Paysandú Province, Uruguay (Rusconi (1933, Soto et al. 2011). Type Horizon—Guichón Formation, Middle Cretaceous (Soto et al. 2011).

Remarks-Originally, Rusconi (1933) distinguished two different species of Uruguaysuchus, $U$. aznarezi (type species) and U. terrai. New material and re-interpretation of the original material by Soto et al. (2011) indicated that $U$. terrai is a junior synonym of $U$. aznarezi. In this work I was unable to include pictures of the type material of Uruguaysuchus and the newly referred specimens are well illustrated in the work of Soto et al. (2011).

\section{General cranial and mandibular morphology}

The newly described skull (FC-DPV 2320, Soto et al. 2011) referred to as Uruguaysuchus is only partially preserved and its dorsal surface is damaged, thus various features can be interpreted only from the holotype specimen (Rusconi 1933). The skull of the small bodied Uruguaysuchus (total body length ca. $90 \mathrm{~cm}$ ) is generally similar to that of other, especially Early or Middle Cretaceous (Malawisuchus, Candidodon, Araripesuchus) or some Late Cretaceous (Sphagesaurus) heterodont notosuchians being relatively high, narrow and triangular in dorsal view, and with approximately straight tooth rows. The rostrum with anteriorly facing external nares is short compared to modern crocodylians (Rusconi 1933). The orbits are facing laterally and slightly anteriorly. The supratemporal fenestrae are large, oval shaped, and anteroposteriorly elongated. The supratemporal shelfs are extended laterally and slightly posteriorly. Behind the triangular bony palate, the oval-shaped suborbital fenestrae are relatively large separated medially by the moderately wide palatines. The triangular choanae are huge and divided, and they are situated between the palatines and the pterygoids. The lateral flange of the pterygoid is anteroposteriorly wide, well developed and 
deeply ventrally oriented similarly to Candidodon or Malawisuchus and it is not as reduced as in Mariliasuchus. The ectopterygoid bordering the suborbital fenestrae laterally is lateromedially wide with a massive posterior process tapering along the lateral pterygoid flange. The temporal region including the origin surfaces of the external adductors is not preserved on the newly described specimen (Soto et al. 2011). However, based on the drawings of Rusconi (1933), the ventral surface of the distal quadrate does not possess any particular ridge or protuberance for the attachement of muscles or tendons. The distal end of the quadrate is oriented ventrally and slightly posteriorly. Based on the morphology of the glenoid surface on the articular (Soto et al. 2011), the two condyles are separated by a shallow groove and the medial condyle of the quadrate is more ventrally projected than the lateral one. The mandible of the newly described specimen is well known. The postdentary part is very high and massive compared to the dentary. In lateral view, the dentary becomes narrower anteriorly and its alveolar margin is slightly waved both in the vertical and horizontal planes. The symphysis is completely fused reaching posteriorly the level of the $9^{\text {th }}$ alveoli. The „caudal intermandibular foramen" anteroventral to the external mandibular fenestra within the splenial described by Iori (2008) in Sphagesaurus and also seen in Yacarerani is not present in Uruguaysuchus. The external mandibular fenestra is huge and roughly triangular shaped. The dorsal surface of the surangular is not as massive and wide as in heterodont notosuchians with palinal movement. The glenoid surface is slightly below the level of the occlusal surface. It is slightly elongate anteroposteriorly ( $75 \%$ of its maximum mediolateral width) and lacks a posterior buttress behind it (Soto et al. 2011). The relative anteroposterior length of the glenoid surface is similar to that of Simosuchus. The glenoid surface is divided into a slightly dorsomedially facing medial articular facet and a dorsally facing lateral facet. The orientation of the medial facet is, however, not as much medially inclined as in Pakasuchus (O'Connor et 
al. 2010). In contrast to Mariliasuchus, the retroarticular process of Uruguaysuchus is very short and projects posteroventrally (Soto et al. 2011).

\section{Dentition and possible extant analogues}

Rusconi (1933) and Soto et al. (2011) gave a detailed description on the dentition of Uruguaysuchus. I have never seen any of the specimens personally, thus here only a short description repeats the dental features of this taxon presented by latter two authors. Uruguaysuchus possesses a heterodont and relatively well regionalized dentition including precaniniforms (incisiviforms of Soto et al. 2011), caniniform, and postcaniniforms. Whereas the anterior teeth and the posterior maxillary teeth are relatively widely spaced, the lower postcaniniform teeth are closely spaced. The upper precaniniform teeth are up to the second maxillary tooth and the lower ones are the first six or seven teeth. They are generally conical, slightly curved lingually, and the enamel surface is smooth with a very faint wrinkle ornamentation (Soto et al. 2011). These teeth appear to bear a distal non-serrated carina. Caniniform tooth is only present in the third maxillary alveolus. It differs from that of other notosuchians in having four apicobasally oriented carinae (Soto et al. 2011). Postcaniniform teeth are labiolingually flattened and mesiodistally carinated, occupying the fourth to $12^{\text {th }}$ alveoli in the maxilla and the eighth to $17^{\text {th }}$ in the dentary. These teeth are mesiodistally longer than high, and oval or subcircular in lateral view. They bear a central, pointed cusp that continues mesiodistally in serrated/cuspidate carinae. The number and size of denticles in the carina is alternating (Soto et al. 2011:fig. 12). Soto et al. (2011) distinguished two different types of occlusal surface among these cheek teeth. In the first type, the central cusp is more developed and the mesial and distal carinae are formed by seven-eight denticles that reach the base of the crown. The second type has weakly developed central cusp with only four, poorly separated denticles in each carina, and the mesial and distal carinae together are almost in the 
horizontal plane to form a single straight, denticulated occlusal cutting blade. This provides a blade-like upper tooth row with relatively widely spaced cheek teeth.

Among crocodyliforms, Araripesuchus spp. possess labioligually flattened teeth with mesiodistally oriented and in some cases denticulated carina (e.g. Ar. wegeneri [Buffetaut 1981] and Ar. gomesii [Buscalioni et al. 2000]). The various species of the atoposaurid Theriosuchus (e.g. BMNH 48262) have labiolingually flattened tooth crowns in the posterior alveoli, but these teeth, although very faintly ornamented on the carinae, are apparently not as denticulated as those of Uruguaysuchus. Only Th. ibericus possesses well serrated carinae mesiodistally (Brinkmann 1992). Some peirosaurid forms (e.g. Montealtosuchus [MPMA-160007-04], Carvalho et al. 2007) also bear posterior teeth similar to Uruguaysuchus but here the serration of the mesiodistal carinae is more developed.

Among extant animals, teeth similar to that of Uruguaysuchus are not common. Some herbivorous forms (e.g. Cyclura, Ctenosaura, Iguana, Sauromalus, see for figures Kley et al. 2010, Ösi 2011) possess labiolingually flattened cheek teeth in the posterior part of the jaws, but in most of these forms the mesiodistal carina bears no denticles but much larger, pointed cusps. The teeth of some species of the insectivorous scincid Eumeces are similar to the cheek teeth of Uruguaysuchus in having an almost horizontal, blade-like occlusal surface, but in this extant lizard this blade is not denticulated or serrated (Kosma 2004).

\section{Dental wear}

The teeth of the new specimen of Uruguaysuchus (FC-DPV 2320) are completely void of any dental wear (D. Pol 2011, pers. com.) and Rusconi (1933) did not mention any wear facet or abraded teeth in the type specimens either, so it can be concluded that in contrast to most other heterodont notosuchians, the upper and lower teeth of Uruguaysuchus did not occlude with each other, similarly to Simosuchus and Araripesuchus. 


\section{Adductor muscle reconstruction}

Only a very rough estimation on the mass and orientation of the cranial adductor musculature of Uruguaysuchus can be done because the type specimens are known only from the work of Rusconi (1933) and from some notes and drawing of Gasparini (1971). Other than that, only the newly described but fragmentary specimen (Soto et al. 2011) can be used for reconstruction of cranial adductor musculature. The anteroposteriorly extended and mediolaterally wide lateral pterygoid flanges, the relatively large, oval suborbital fenestrae and the high and massive postdentary part of the mandible indicate extended origin and insertion surfaces for the pterygoid muscles, i.e. the relative development of these internal adductors were comparable to that of modern crocodylians. The ventral surface of the quadrate-quadratojugal complex is only known from Rusconi (1933), so the origin surfaces of MAMP, MAMEM and MAMES are hard to interpret. The deep postdentary part of the mandible, however suggests that along with the developed MPTD attaching here, a relatively large mass of MAMP was also inserted in the same area. The relatively thin surangular emarginating dorsally the external mandibular fenestra indicates that MAMEM and MAMES could not have been of crucial importance during jaw closure. Based on Rusconi’s (1933) illustration, Uruguaysuchus possesses a large, oval-shaped, anteroposteriorly more elongate supratemporal fenestra extending medially on the parietal. The size of the supratemporal fenestra indicates a relatively well developed MAMEP in this taxon. It can be assumed that, in contrast to other Late Cretaceous South American heterodont notosuchians (Mariliasuchus, Yacarerani, sphagesaurids), the jaw adductors of Uruguaysuchus were more similar to those of modern crocodylians in having well developed, powerful pterygoid muscles and not so specialized and less developed external adductors. 


\section{Jaw mechanism and possible phases of chewing}

Although the craniodental features of Simosuchus and Uruguaysuchus show important differences, numerous features as indicators of the mechanism of mandibular movement are similar. Although markedly heterodont, the dentition of Uruguaysuchus does not show any evidence of dental wear on any of the teeth. This unambiguously indicates that no dental occlusion (i.e. well-controlled tooth-tooth contact) was present in Uruguaysuchus. Uruguaysuchus possesses an anteroposteriorly slightly elongated glenoid surface (it is far from the relative length of the glenoid surface seen in heterodont notosuchians) with no buttress behind it, and the mandibular symphysis is completely fused, similar to Simosuchus. This could have allowed a limited anteroposterior movement of the mandibles during powerstroke. However, the absence of precise tooth-tooth contact indicates that, similarly to Simosuchus, a proal or palinal movement to provide more efficient oral food processing did not exist in Uruguaysuchus. If there was any anteroposterior sliding of the mandibles during powerstroke, then it could only have provided a wider gape or helped to fix the correct relative positioning of the upper and lower tooth rows, as it was noted e.g. in nemegtosaurid sauropods (Upchurch and Barrett 2000).

On the other hand, Uruguaysuchus and Simosuchus show also a great number of differences related to jaw mechanism. In Simosuchus, the quadrate is ventrally and slightly anteroventrally oriented, the pterygoid flanges are reduced and anterolateroventrally oriented, the quadrate-mandible joint is far deep below the occlusal plane, and the external adductors are particularly developed. In contrast to Simosuchus, Uruguaysuchus has a ventrally and slightly posteriorly oriented quadrate and the glenoid surface is only slightly below the level of the occlusal plane. In addition, their dentition is also markedly different. Whereas in Simosuchus the cuspidate teeth rather resemble the teeth of some herbivorous lizards (Kley et 
al. 2010), posterior cheek teeth of Uruguaysuchus form a serrated cutting blade to provide a shearing mechanism in which the upper and lower teeth did not occlude with each other.

\section{Heterodont neosuchians}

One of the most successful specializations of the heterodont crocodyliform dentition is the development of bulbous crushing teeth in the posterior part of the tooth row. Although typical, globular crushing teeth occured first among neosuchians no earlier than the Early Cretaceous, this functional specialization of the tooth row developed in various lineages during the evolution of the Neosuchia. Among neosuchians, there is a great number of forms (e.g. Goniopholis BMNH R4400, R 1901, 37972, Allodaposuchus (Delfino et al. 2008) Diplocynodon BMNH 30394) that bear still not globular but massive, stocky, although relatively small teeth posteriorly. Although these teeth are usually heavily worn apically indicating the frequent crushing of hard food item (but not tooth-tooth contact), they rather show a conical form and are widely spaced in the jaw. (Similarly massive, low crowned posterior teeth are also present in some, carnivorous notosuchians). Forms with this massive, conical back teeth are usually large bodied and their general cranial and mandibular architecture is robust with a great mass of jaw adductors (especially the MPT were highly developed). Regarding their feeding habits, these forms could have been rather generalists and quite similar to modern crocodylians so they are not discussed in this work.

Posterior crushing teeth in the back of the jaws with relatively closely spaced arrangement evolved already among some non-eusuchian neosuchians (e.g. Unasuchus, Bernissartia). Their teeth are still small relative to skull length, low crowned and labiolingually slightly flattened with rounded occlusal surface. These forms will be compared with the typical durophagous, globidont forms. 
Globular, relatively large-sized and closely spaced crushing teeth are widespread among alligatoroid eusuchians (e.g. Stangerochampsa, Brachychampsa, Albertochampsa, Diplocynodon deponiae, Allognathosuchus; Erickson 1972, Brochu 1999, Wu et al. 1996, Delfino and Smith 2012) including some extant species (Caiman latirostris, Ösi and Barrett 2011). In addition, Osteolaemus has to be mentioned also the two posterior teeth of which are blunt and almost hemispherical (Buffetaut and Ford 1979). These forms will be compared and discussed here in detail. Besides these forms with simple bulbous crushing teeth, the hylaeochampsid form Acynodon (Buscalioni et al. 1997, Martin 2007, Delfino et al. 2008, Rabi and Ösi 2010) and Iharkutosuchus (Ösi et al. 2007, Ösi 2008, Ösi and Weishampel 2009) are exceptions because they bear a slightly (Acynodon) or highly specialized (Iharkutosuchus) dentition, thus they will be discussed in different chapters.

\section{Heterodont neosuchians with crushing teeth}

Neosuchia Clark, 1986

Studied genera-Unasuchus, Bernissartia, Brachychampsa, Allognathosuchus, Caiman latirostris, Osteolaemus tetraspis.

Time range_-Early Cretaceous to present.

Remarks - Because these crocodyliforms with bulbous crushing teeth show great similarities in most of the important cranial and mandibular characters related to feeding, in the following chapter I discuss them together and note the main differences between the individual taxa.

\section{General cranial and mandibular morphology}

The skull of these small to medium-sized crocodyliforms with crushing teeth is brevirostrine and platyrostral (sensu Busbey 1995) with generally massive and rounded rostrum. The 
brevity of the rostrum apparently reflects the size of the posterior crushing teeth relative to skull size. In the non-eusuchian Bernissartia fagesii (Dollo 1883, Buffetaut and Ford 1979, Buscalioni and Sanz 1990, Figure 43), the teeth are relatively small, still labiolingually flattened and the rostrum is triangular, slightly waved, and still pointed. Although known only from fragmentary material (premaxilla, maxilla, occipital region, quadratum, mandible, teeth), based on the reconstruction of Brinkmann (1992), Unasuchus reginae possessed a much shorter and wider rostrum than did Bernissartia (IRScNBr 46). In the extant Caiman latirostris, the rostrum is slightly broader (Figure 44) than that of Bernisartia and Osteolaemus tetraspis Cope, 1861 (BMNH 61.3.20.8) (Ősi and Barrett 2011). In Brachychampsa montana (UCMP 133901), it is even wider, massive, and rounded anteriorly (Gilmore 1911, Norell et al. 1994, Sullivan and Lucas 2003). Among these forms, Allognathosuchus haupti possesses the relatively shortest rostrum (probably similar to that reconstructed in Unasuchus) with the relatively largest globular crushing teeth posteriorly (Figure 45). In sharp contrast with the functionally heterodont and terrestrial notosuchians presented above, these neosuchians with crushing teeth were/are semiaquatic animals thus their platyrostral skull bears dorsally or slightly laterodorsally facing orbits, dorsally facing external nares, and much smaller supratemposral fenestrae relative to skull size. In addition, the orbits are generally smaller in these neosuchians than in heterodont notosuchians (Mariliasuchus, Yacarerani). Relative to skull size, Allognathosuchus possesses the largest orbit which is proportionally about the same size as that of heterodont notosuchians. With the small supratemporal fenestrae, the size of the whole skull table is also smaller (except for Osteolaemus which possesses a relatively large skull roof with small supratemporal fenestrae). On the other hand, the marginal area lateral to the skull table (quadratequadratojugal-lateral temporal fenestra complex) became wider and massive. This is 
especially prominent in Brachychampsa (UCMP 133901, Norell et al. 1994). The lateral temporal fenestrae are triangular and usually relatively large.

The bony palate of heterodont neosuchians with crushing teeth is usually a wide and hardly strengthened structure especially around the posterior crushing teeth, definitely to resist torsional stresses during bite (Clark and Norell 1992). The posterior process of the maxilla lateral to the suborbital fenestrae (especially in Bernissartia, Allognathosuchus [Lucas and Estep 2000] and Caiman latirostris) is wide and massive medially (Figures 43, 44, 45). The ectopterygoids, although in alligatoroids there is no direct contact with the alveoli (Norell et al. 1994), are robust to support the region including the large and wide globular teeth. In Brachychampsa, Allognathosuchus and moderately in Bernissartia, the palatinal bridge between the suborbital fenestrae is wide compared to the skull width. Similarly to hylaeochampsids (Ösi et al. 2007), the prefrontal pillar is massive in these forms and unusually wide in Brachychampsa (Norell et al. 1994). Posteriorly, the massive palate is ended in the pterygoids which in all forms possess large and extended lateral pterygoid flanges. In spite of the broad surrounding bones, the suborbital fenestrae are moderate (Allognathosuchus, Brachychampsa) to large sized (Bernissartia, Caiman latirostris) compared to skull size. In Osteolaemus these fenestrae are approximately one third of the total skull length. The quadrate is wide mediolaterally in Bernissartia (Figure 43(B)), Brachychampsa, and Caiman latirostris (Figure 44(B)), and the ventral surface of its shaft in the latter two taxa bears developed crests for the attachment of jaw adductors (Figure 44(D)). The quadrate is usually short posteriorly (see e.g. Allognathosuchus mooki [Lucas and Estep 2000], Osteolaemus). In contrast to the markedly posteroventrally or in some cases ventrally oriented distal end of the quadrate seen in most heterodont notosuchians, it is oriented posteriorly and only slightly ventrally in these neosuchians. The two condyles of the quadrates are well separated by a shallow intercondylar groove. The consequence of the distal 
quadrate orientation is that the articular surface of the two condyles is not ventrally positioned as in most heterodont notosuchians (e.g. Mariliasuchus, Sphagesaurus, Yacarerani or Candidodon) but posteriorly or posteroventrally.

As generally seen in all neosuchians, the glenoid area on the mandible in thee forms with crushing teeth is a deeply concave, divided surface bordered posteriorly by a relatively high transverse ridge (Figure 44(E)). This feature together with the high posterior margin of the surangular prevents any significant anteroposterior or transverse movement. In neosuchians with crushing teeth, the quadrate-mandible articulation is well above the occlusal surface, in contrast to the heterodont notosuchians. On the other hand, the postdentary part of the mandible accepting the jaw adductors is always high in both heterodont notosuchians and neosuchians. The retroarticular process is relatively elongate, triangular and dorsally concave in contrast to the posteromedioventrally oriented process of heterodont notosuchians. The external mandibular fenestra is relatively large in Brachychampsa, Allognathosuchus haupti [HLMD ME 4415] and Caiman latirostris, it is small in Allognathosuchus mooki (Lucas and Estep 2000) and completely closed in Bernissartia and Unasuchus. The mandible is more robust and strongly built in Allognathosuchus (Lucas and Estep 2000) and Brachychampsa (Carpenter and Lindsay 1980) than in Bernissartia, Unasuchus, Osteolaemus and Caiman latirostris. The mandibular symphysis is massively fused in Brachychampsa, Osteolaemus and Caiman latirostris but apparently unfused in Bernissartia and Allognathosuchus (Lucas and Estep 2000).

\section{Dentition and possible extant analogues}

The dentition of neosuchians with crushing teeth is heterodont but the regionalization of the functionally different teeth is not as complex and prominent as in various heterodont notosuchians (e.g. Pakasuchus, O'Connor et al. 2010). In most cases, the transition between 
the anterior conical and postrior crushing teeth is graduate showing some transitional, low crowned but not so large and globular, still conical transitional teeth. The dentition of neosuchians with crushing teeth is generally similar to each other, and differences can be pointed out only in the number of teeth and in the shape and size of the globular back teeth. In all of these taxa, the anterior and middle parts of the tooth row are composed of conical, pointed teeth that have circular or subcircular cross-section and possess unserrated carinae mesiodistally (Figure 43(C), 44(F), 45(D)). These teeth are usually medium to small sized. Larger, caniniform teeth appear in all taxa both in the upper and lower tooth rows but in slightly different ways. For example, whereas Allognathosuchus mooki (Lucas and Estep 2000) and Caiman latirostris possess an enlarged caniniform in the fourth maxillary alveolus, Brachychampsa has a large caniniform in the fifth position (Norell et al. 1994). On the other hand, Bernissartia has two large upper caniniforms in the anterior part of both maxillae (Buffetaut 1975). Concerning the lower jaw, Allognathosuchus haupti bears an enlarged caniniform tooth anteriorly (the largest in the lower tooth row) that is followed by numerous, much smaller conical teeth in a sinusoidal tooth row seen in lateral aspect. In the middle of the tooth row, another larger conical tooth occurs that is followed by some smaller conical teeth just in front of the posterior crushing teeth. The lower tooth row of Allognathosuchus mooki slightly differs from that of Allognathosuchus haupti in having two neighbouring large alveoli in the middle of the tooth row. A similar configuration can also be observed in Brachychampsa.

Whatever is the configuration of the smaller and larger conical teeth in these heterodont neosuchians, their last two (in Osteolaemus, Buffetaut and Ford 1979) to seven (in Allognathosuchus mooki, Lucas and Estep 2000) alveoli uniformly bear low crowned, stocky or globular teeth with finely wrinkled enamel cover (Figure 45(D-J)). These teeth are not completely hemispherical, but slightly labiolingually flattened, and rather oval shaped in 
Allognathosuchus (Rossmann 2000) and Osteolaemus. In Unasuchus (Figure 47(A)), Bernissartia (BMNH R 9296, Buffetaut and Ford 1979:plate 122, fig. 2; Figure 45(D-J)) and Brachychampsa (Sullivan and Lucas 2003), some of the teeth are more rectangular in occlusal view. Among these globular teeth in Bernissartia, Allognathosuchus mooki (Lucas and Estep 2000), Al. haupti, Brachychampsa (Sullivan and Lucas 2003), the penultimate tooth is the largest. These posterior teeth are very closely spaced to each other (Figure 45(H), 46(A)) but usually there is no contact between the crowns (in contrast to the posteriror teeth of Iharkutosuchus, see below). Contrary to the other forms with posterior crushing teeth, the largest blunt tooth in the maxilla of Caiman latirostris is not the penultimate but the fourth tooth from posteriorly (Figure 48(A, B)). This tooth is actually not as bulbous as in Allognathosuchus or Brachychampsa, but more pointed. Crushing teeth are generally constricted between the crown and the root. The root of these teeth is massive and frequently close to rectangualar in cross-section.

Posterior crushing teeth are relatively frequent among extant lizards (e.g. some species of Dracaena, Tupinambis, Hemisphaeriodon, Varanus, Tiliqua, Ameiva, Cyclodomorphus, Novoeumeces, for a review see Kosma 2004) usually charcterized by a durophagous or omnivorous diet. However, as reported by Ösi and Barrett (2011, Figure 48), two extant forms of the Crocodylia, Caiman latirostris and Osteolaemus tetraspis also bear globular crushing teeth posteriorly. These species are also regarded as durophagous or omnivorous.

\section{Dental wear}

Dental wear facets frequently occur in neosuchians with crushing teeth. Sometimes they are present on the anterior or middle conical teeth too (e.g. on the $14^{\text {th }}$ maxillary tooth of Brachychampsa, Sullivan and Lucas 2003, or on the carinae and apex of the anterior teeth of Caiman latirostris) but they are most developed on the posterior crushing teeth. In some 
cases, teeth have completely lost their enamel coating all over the crown (e.g. Unasuchus, Caiman latirostris) and they have been worn down into a wide, almost flat or slightly sloping crushing surface formed only by dentine (Ösi and Barrett 2011). In Bernissartia, Brachychampsa, Allognathosuchus, and Osteolaemus, posterior teeth are not as eroded as in the former taxa, although in many cases the dentine is rather exposed. Wear facets are heavily ornamented with numerous pits and short scratches. Macrowear patterns are mostly observed on the dentine and they are dominated by irregular or rarely circular pits. In some cases (e.g. in Brachychampsa), especially more labial or lingual to the apex, the eroded enamel surface is smoother with fewer pits and thinner scratches (Figure 47(E)). Pits are frequently over $1 \mathrm{~mm}$ in diameter and possess coarse surface. Scratches are usually short, wide, and V-shaped in cross-section. Except for some wear facets on the teeth of Unasuchus (Figure 47(C)), they do not show any preferred orientation. EDI is usually irregular. In some cases, the dentine is deeply eroded (e.g. Bernissartia, Figure 46(B, D-G)) but sometimes the enamel coat is crushed and only a conical dentine stump is present (Caiman latirostris, Ösi and Barrett 2011, Figure 48(C, F)).

\section{Adductor muscle reconstruction}

The jaw adductors of the neosuchians with crushing teeth are generally similar to those of other neosuchians including modern forms (e.g. Alligator mississippiensis, Figure 4). It can be unambiguously demonstrated that the most developed jaw adductors of neosuchians with crushing teeth were the pterygoid muscles. The large suborbital fenestrae (especially for example in Osteolaemus) and the massive surrounding bones indicate an extensive origin surfaces for MPTD and suggest that MPTD was one of the most dominant jaw adductors. In the extant Alligator mississippiensis, the suborbital fenestrae are similar in size to those seen in the studied neosuchians with crushing teeth, and the MPTD provides $18.34 \%$ of the total 
jaw muscle mass being the second largest jaw adductor (Busbey 1989:table 2). So, it is supposed that the development of MPTD in these taxa was comparable or in some forms perhaps more developed than in Alligator mississippiensis. Based on the extended pterygoid flanges both anteroposteriorly and lateromedially, and the great insertion surface on the posterolateral part of the angular (especially in Allognathosuchus haupti), MPTV could have been the most dominant jaw adductor in these forms, as it was demonstrated also in Alligator mississippiensis (34.48\% of the total jaw muscle mass, Busbey 1989). The relatively short and wide ventral surface of the quadrate shaft sometimes ornamented with Iordansky's (1964) crests in addition to the deep postdentary part of the mandible indicate great mass of MAMP that is comparable to the proportional mass of that of Alligator mississippiensis $(15.34 \%$ of the total jaw muscle mass, Busbey 1989).

Regarding the external adductor muscles, MAMES and MAMEM represent $24.84 \%$ and MAMEP only $2.94 \%$ of the total mass of jaw adductors (Busbey 1989). The origin surfaces of MAMES and MAMEM (i.e. the lateral margin of the ventral surface of the quadrate shaft and the ventral surface of the quadratojugal) are at least as wide in these taxa as in Alligator mississippiensis, and their insertion surface (dorsal and dorsomedial surface of surangular) is also wide and massive. This refers to a MAMES and MAMEM comparable in mass to that of Alligator mississippiensis. In Brachychampsa, the exceptionally wide ventral surface of the quadrate-quadratojugal complex may suggest an even more developed MAMES-MAMEM complex than that in other forms. Based on the relatively small to medium size of the supratemporal fenestrae and narrow supratemporal fossae compared to the skull length, MAMEP and MPSS could have been week and not really important muscles in sharp contrast to heterodont notosuchians with palinal movement. Assuming the information on the jaw adductors of neosuchian crocodyliforms with crushing teeth, these animals possessed a 
generally similar construction of jaw adductor musculature compared to Alligator mississippiensis (Busbey 1982, 1989).

\section{Jaw mechanism and possible phases of chewing}

On the basis of the precise and anteroposteriorly short quadrate-mandible joint, the wide lateral pterygoid flanges, the absence of fine, continuous enamel-dentine interfaces, and the lack of long scratches on the wear facets, it is evident that neosuchian crocodyliforms with crushing teeth can be charaterized with a simple but precise, isognathous orthal jaw closure

just as all extant crocodylians (Figure 49). The lateromedially oriented posterior buttress behind the glenoid surface certainly prevented any significant anteroposterior shifting of the mandibles. The anteroposterior shortness of the glenoid surface, the high posterodorsal part of the surangular, the wide lateral pterygoid flanges, and in some cases, the fused symphysis did not allow the mandibles to translate or even to rotate lateromedially. The anterior dentition including large caniniform teeth shows that all these forms could have been opportunistic predators.

In extant crocodyliforms, including Caiman latirostris, there is no occlusion of the upper and lower teeth (Figure 48(A)). This means that the extensive wear facets with coarse pits and scratches seen on the teeth of C. latirostris (NHM RR 2009.1-2) were produced by crushing of hard-shelled food item and not by regular tooth-tooth occlusion (Ösi and Barrett 2011). Based on two articulated specimens of Osteolaemus tetraspis (NHM 1957.1.4.42. and NHM 61.3.20.8.), the absence of dental occlusion can be exluded also in this genus, so the apical wear present on its teeth were certainly the result of tooth-food-tooth contact, similarly to $C$. latirostris. I suggest that this could have been the case in Bernissartia because the lower teeth are positioned well medially to the upper one preventing any tooth-tooth contact, and the teeth of Bernissartia bear usually apical wear and no labially or lingually positioned, 
obliquely oriented wear facets characteristic of precise tooth-tooth occlusion. However, in Unasuchus, Brachychampsa and Allognathosuchus, it is not completely clear whether direct tooth-tooth contact between the upper and lower teeth was present or not. In Unasuchus, the cranial material (premaxilla, maxilla, and mandible) is too fragmentary to reconstruct the original position of the upper and lower tooth rows relative to each other. On the other hand, among fossil forms with globular teeth, only Unasuchus shows extended and well oriented, flat wear facets (Figure 47(A-D)). Similar, slightly sloping flat wear facets occur, for example, in Iharkutosuchus. However, on some of the worn maxillary teeth of Caiman latirostris (NHM RR 2009.1-2), wear facets are also extensive, flat and slightly medioventrally facing as seen on the isolated teeth of Unasuchus, and in spite of that there is no dental occlusion in C. latirostris. An important difference between the wear facets of Unasuchus and C. latirostris is the presence of some elongate, parallel scratches in the former taxon (Figure 47(D)). In the case of Unasuchus, more complete material is needed to precisely reconstruct the position of the upper tooth row relative to the lower one. In the most complete specimen of Brachychampsa (Norell et al. 1994:fig. 5), the upper globular teeth are apparently lateral to the lower tooth row, so in this preserved configuration they are not in an occluding position. Study of the wear facets on isolated teeth of Brachychampsa shows that its bulbous teeth bear some wear facets with peferred orientation. This may suggest the possibility of tooth- tooth occlusion and thus a more efficient oral food processing compared to other neosuchians with crushing teeth but, again, more material is needeed to support this hypothesis.

The only taxon with certainly occluding bulbous crushing teeth preserved is Allognathosuchus haupti. In the specimen HLMD ME 3094, the slightly convex occlusal surface of the left posteiror crushing teeth are in an occluding and interdigitating configuration where the crown of an upper tooth fits within the concave surface between the crowns of the two lower teeth 
(Figure 45(E)). In addition, Allognathosuchus haupti exhibits the largest crushing teeth relative to its skull. Supposing that this articulated configuration represents in vivo position of the upper and lower tooth rows, it can be concluded that in Allognathosuchus the upper and lower crushing teeth certainly occluded with each other (Figure 49(C-E)), and that food processing could have been the most efficient in this form among neosuchians with crushing teeth.

Due to the absence of complex jaw mechanism, the different phases of food processing in neosuchians with crushing teeth were most probably quite similar to that of extant crocodylians. After capturing the prey item with the anteriorly positioned conical and slightly lingually curved teeth, the food was shifted posterolaterally partially by the tongue protruding between the upper and lower crushing teeth and also with the help of the fast upward movements of the head as seen in extant crocodylians. In this preparatory stroke, the role of the external adductors (parts of the MAME and MPS) could have been the most important, because during prey capture fast and repeated jaw closure is essential. The powerstroke could have been relatively simple and it was not divided into different phases as in heterodont notosuchians with complex jaw movements. During the powerstroke, the food was triturated and crushed by the massive posterior crushing teeth within several cycles of isognathous orthal closure. The activity of pterygoid muscles and probably of MAMP could have been the highest in this phase.

Eusuchia Huxley, 1875

Hylaeochampsidae Andrews, 1913

Hylaeochampsidae appears to have been an endemic European group of basal eusuchians. Until the discovery of Iharkutosuchus makadii, only the Barremian Hylaeochampsa vectiana 
Owen, 1874 was the single member of the family. Phylogenetic analysis of Ösi et al. (2007) indicted that Iharkutosuchus makadii is also a member of this group having a short and constricted rostrum in front of the orbits and enlarged crushing-grinding teeth in the posterior alveoli (the teeth of Hylaeochampsa are unknown). Rabi and Ösi (2010), Turner and Brochu (2010) and Brochu (2011) recognized that the western European Late Cretaceous Acynodon shares several features with the Hylaeochampsidae and so they assigned Acynodon into this group rather than to alligatoroids (contra Martin 2007, Delfino et al. 2008). Most importantly, Acynodon posssesses a complex dentition with spatulated anterior and large, bulbous posterior teeth the latter of which, however, are more complex than the globular teeth of other neosuchians discussed above. The teeth of Acynodon along with the reconstructed jaw mechansim are markedly different from that of Iharkutosuchus, so both hylaeochampsid are discussed in a different chapter.

Iharkutosuchus makadii Ösi, Clark and Weishampel, 2007

(Figure 50-56)

Holotype-Nearly complete skull (MTM 2006.52.1.).

Referred specimens_-nearly complete skull (MTM 2006.53.1.), three partial skulls (MTM 2006.54.1., MTM 2006.55.1., MTM 2006.56.1.), three skull fragments (MTM 2006.57.1., MTM 2006.65.1., MTM 2006.66.1.), several different isolated skull elements (MTM 2006.67.1., MTM 2006.68.1., MTM 2006.79.1., MTM 2006.69.1., MTM 2006.73.1., MTM 2006.77.1.), a complete left mandible (MTM PAL2012.29.1.), a fragmentary right mandible (MTM 2006.58.1.), 12 isolated fragmentary mandibles (MTM 2006.58.1.-2006.64.1., MTM 2006.71.1., 2006.72.1., 2006.74.1.-2006.76.1.), three mandible fragments (MTM 2006.78.1., MTM 2006.70.1., MTM PAL2012.30.1.) and 148 isolated teeth (MTM 2006.80.1.). 
Type Locality-Iharkút, Bakony Moutains, western Hungary, Central Europe (Ősi et al. 2007).

Type Horizon—Csehbánya Formation, Upper Cretaceous, Santonian (Knauer and SieglFarkas 1992, Szalai 2005).

Remarks - Ösi et al. (2007) and Ösi (2008) gave a detailed description of the cranial and dental anatomy of Iharkutosuchus. In addition, Ösi and Weishampel (2009) reconstructed the jaw mechanism and dental function of Iharkutosuchus, thus only a short summary of these studies is provided here including some new information related to the quadrate-mandibular joint.

\section{General cranial and mandibular morphology}

Iharkutosuchus possesses a small $(11.1 \mathrm{~cm})$, brevirostrine and platyrostral skull with dorsally facing confluent external nares and dorsally and slightly dorsolaterally facing, anteroposteriorly more elongate orbits (Figure 50(A)). This taxon is characterized by the reduction of cranial openings both dorsally and palatally. Antorbital and supratemporal fenestrae are closed even in subadult animals (Ösi et al. 2007). The temporal region, including the lateral temporal arcade, is mediolaterally wide with a moderately sized, triangular lateral temporal fenestra. Ventrolateral to the small orbits, at the level of the massive maxilla-jugal complex, the skull abruptly widens. In the secondary bony palate, the suborbital fenestrae are narrow and anteroposteriorly elongate bordered medially by a narrow palatinal bridge and laterally by the abruptly widening and massive ectopterygoids (Figure 50(B)). In contrast to alligatoroids (Norell et al. 1994), the ectopterygoids border the last two alveoli and support the massive roots of the teeth. In contrast to most crocodyliforms, the pterygoids have reduced lateral flanges but extremely elongate (approximately $2 \mathrm{~cm}$ ) posterior processes with a posteroventral orientation. The quadrate is lateromedially wide and extremely short 
anteroposteriorly. It is shorter than that of Hylaeochampsa and even shorter than that of Acynodon and more similar in length to that of Allognathosuchus haupti (Lucas and Estep 2000). Ventrally, the quadrate shaft bears a huge (1 cm in diameter), triangular-shaped, almost $5 \mathrm{~mm}$ high protuberance, certainly for the extension of muscle attachments (Figure 50(B), see below). Quadrate condyles are separated by a wide intercondylar groove and show a slightly twisted configuration as in many crocodyliforms. The medial condyle is more process like with posteroventral orientation and the lateral condyle has an almost posteriorly facing aricular facet. The lateral temporal arcade is narrow, but the quadratojugal is wide and massive forming a relatively great part of the dorsolateral segment of the temporal cavity. Although slightly broken, the occipital condyle appears to be oriented posteroventrally. In the description provided by Ösi et al. (2007) and Ösi (2008), the mandible of Iharkutosuchus was discussed in detail based on 13 fragmentary mandibles, but in 2010 a new and complete left mandible (MTM PAL2012.29.1.) has been discovered including the posterior teeth (Figure 50(D-F)). This specimen bears a number of additional important features so the description of the mandible presented here is based on this specimen. Its size (including that of the teeth) fits well to the largest (holotype) skull, so most probably it represents an adult animal. The symphysis is anteroposteriorly elongate, slightly anterodorsal-posteroventrally oriented, and posteriorly it reaches the level of the $9^{\text {th }}$ alveoli. Neither the previously described 13 fragmentary mandibles nor this specimen shows any indication of fused mandibles at the symphysis. This is further supported by the fact that in most cases the medial, ornamented surface of the symphysis is always clear and well preserved. Ősi et al (2007) noted 14 alveoli and later Ösi (2008) counted 15 alveoli in the dentary. However, in the previously described 13 fragmentary mandibles some parts of the alveolar region are filled with pyrite or the anterior symphyseal end is broken so the total number of the alveoli was mostly estimated. This clear and complete, new specimen allows 
the recognition of altogether 16 alveoli in the dentary among which the anterior 9 are certainly separated from each other by bony septa (Figure 50(E)). The splenial, previously unknonwn element of the mandible, is a massive element that supports the robust roots of the last 7 teeth and forms the anteromedial margin of the mandibular adductor fossa. In contrast to some globidont forms, the dorsomedial part of the splenial is not widened to give a greater support for the posterior grinding teeth. The „,caudal intermandibular foramen” anteroventral to the external mandibular fenestra within the splenial described by Iori (2008) in Sphagesaurus and also seen in Yacarerani is not present in Iharkutosuchus. From the $10^{\text {th }}$ alveoli, the lateral wall of the dentary becomes abruptly wide to give a massive basement of the grinding teeth, and the shelf present lateral to the tooth crowns most probably served as attachment area for a bucca. The surangular is short with a very short, arched, laterally convex and edge-like dorsal margin (Figure 50(E)). The glenoid surface is highly above the occlusal plane. The glenoid surface is similar to that of extant crocodyliforms in having an elevated posterior buttress behind it and that its articular surface is not horizontal as in heterodont notosuchians but is facing slightly anterodorsally. However, the new specimen clearly indicates some important differences compared to that of extant forms. With $40 \%$ of its total length, the glenoid surface well extends on the distal surface of the surangular. The facet for the lateral quadrate condyle is $14.8 \mathrm{~mm}$ wide, more than two times wider than the facet for the medial condyle (Figure 50(E)). In extant forms (e.g. Crocodylus sp., Alligator mississippiensis), the quadrate condyles are as wide lateromedially as the glenoid surface, and the glenoid surface is approximately 1.5 times longer anteroposteriorly than the quadrate condyles. In Iharkutosuchus, however, the glenoid surface is $2-2.5$ times longer, especially on its lateral side, than the quadrate condyles. This further supports the complex jaw mechanism suggested in Iharkutosuchus (Ösi and Weishampel 2009, see below). The retroarticular process is robust and concave dorsally with a smooth dorsal surface. 


\section{Dentition and possible extant analogues}

The dentition of Iharkutosuchus is the most complex among neosuchian crocodylifoms in having spatulate incisiviform and multicusped molariform teeth (Figure 51). The tooth row has an S-shaped curvature in occlusal view. The enamel surface of the teeth is ornamented by wrinkles that are well developed on the posterior grinding teeth. Teeth are closely packed so that the crowns of the last four-five teeth sometimes contact each other. Because tooth number (18 teeth in the upper and 16 in the lower jaws) is high compared to heterodont notosuchians, regionalization of different teeth is not so pronounced as, for example, in Pakasuchus (O'Connor et al. 2010). Posteriorly, the spatulate incisiviforms gradually transform into more flat teeth with cingulum (Figure 51(C-E)), and these transitional teeth transform into the multicusped teeth in the posterior segment of the tooth row (Figure 51(F, $\mathrm{G})$ ).

Of the complete upper tooth row reconstructed by Ösi (2008:fig. 9), only the last eight teeth are preserved in situ. The anterior part of the upper tooth row was reconstructed on the basis of various isolated teeth that fit well in size, general morphology and wear pattern to the in situ teeth (Figure 51(A, B)). The five premaxillary teeth have been reconstructed as spatulated incisiviform teeth with only minor size difference. These teeth possess a high, cutting edgelike labial part and a more shelf-like lingual part but no cingulum or cusp occurs on any part of the crown. The first six maxillary teeth are roughly similar in shape to the premaxillary teeth, but on the lingual margin they bear a developed and crenelated cingulum and their labial, spatulated part becomes more massive and divided into three cusps among which the central one is the largest. The following four teeth are slightly larger, labiolingually wider but with lower crowns, and the cingulum transforms into a row of cusps on the first two teeth and into two parallel, mesiodistally oriented rows of cusps on the next two teeth. Here, the labially 
positioned main row of cusps is composed of a central cusp and two smaller mesial and distal cusps. Whereas the anterior six maxillary teeth are subcircular in shape, the more posterior teeth are rectangular, trapezoidal in occlusal view (Figure 51(G)). The last three teeth in the maxilla show slightly different morphology compared to the anterior ones and to each other. The $16^{\text {th }}$ tooth is still trapezoidal, and it possesses a main row of cusps but on its lingual shelflike part, a high number of small cusps are ordered into four-five transversely and radially oriented rows of cusps. The penultimate tooth is the largest (anteroposterior length: $11.1 \mathrm{~mm}$ ) having a low main row of cusps including a central cusp and numerous smaller cusps mesiolingually and distolabially. Secondary rows of cusps are on both the labial and lingual sides of the crown being radially oriented and composed of numerous small, stocky secondary cusps. Because of its size, the labial margin of this tooth is much more labially positioned, so this gives the posterior part of the tooth row a strong labial curvature. The last tooth being even more labially positioned is drop-shaped in occlusal view with a roughly transversely oriented and slightly curved central row of cusps that is surrounded distally and mesially by a few secondary cusps.

Previously the lower tooth row was known only on the basis of isolated teeth and its reconstrucion by Ösi (2008) suggested a generally similar composition and tooth morphology to that of the upper tooth row. The anterior dentary teeth were most probably similar to that of the premaxillary teeth in having non-cuspidate, spatulate incisiviform teeth with labially positioned high cutting edge. Similarly to the upper tooth row, posteriorly the spatulate crown becomes lower and bear a cingulum but only on the labial side. In the middle of the lower tooth row, the cingulum is replaced by a row of small cusps. The new, complete left mandible (MTM PAL2012.29.1.) with only the last five teeth preserved (Figure 50(D-F)) and also a right, smaller mandible fragment (MTM PAL2012.30.1.) with the $14^{\text {th }}$ and $15^{\text {th }}$ teeth preserved confirm that the general morphology of the posterior teeth is quite similar to that of 
the upper teeth. However, the position of the rows of cusps, in addition to the number and orientation of cusps, is hard to interpret because the tooth crowns are completely worn (no enamel cover is preserved). In spite of that, the morphology of the preserved crown suggests that the $12^{\text {th }}$ tooth had a main row of possibly three cusps lingually and that the secondary row of cusp was on the labial side. The $13^{\text {th }}$ tooth was similar but the crown is slightly wider on its distal part. These teeth are rectangular but mesiodistally more elongate than their labiolingual width. The $14^{\text {th }}$ tooth is trapezoid and two times wider than the $13^{\text {th }}$ tooth. Following the pattern of the upper tooth row, the $15^{\text {th }}$, penultimate tooth is the largest in the dentary being slightly smaller than the $13^{\text {th }}$ in the maxilla but similar in shape. It seems that both the $14^{\text {th }}$ and $15^{\text {th }}$ teeth possess their main row of cusps on the lingual side of the crown and most of the secondary rows of cusps are situated labially but their details cannot be observed. The last, $16^{\text {th }}$ tooth is in a higher, slightly oblique position (Figure 50(D)) compared to the more anterior teeth, and its worn crown faces mesiodorsally. It is not as labially positioned relative to the penultimate tooth as the last tooth in the maxilla and its central main row of cusps is not transversely but mesiodistally oriented and bordered both labially and lingually by secondary cusps.

\section{Dental wear}

Dental wear is a characteristic feature of every erupted tooth of Iharkutosuchus indicating a very intensive use of them. Wear pattern including macrowear on both the enamel coat and the dentine and microwear on the enamel have been described in detail in Ösi and Weishampel (2009), so here only a short summary of the previous results is given. In addition, new data on the wear facets of the lower teeth based on the new specimens (MTM PAL2012.29.1., MTM PAL2012.30.1.) is also provided. The anterior incisiviform teeth possess extensive wear on the occlusal surface of their cutting-edge like labial part. This 
surface is usually flat or slightly convex or concave, and they are rarely perpendicular to the long axis of the crown but most preferentially obliquely positioned (Figure 52). On the worn surface, besides small pits, both labiolingually and mesiodistally oriented scratches occur. These teeth were never found in situ so it is unclear which orientation of the wear facets refer to which position of the tooth row.

Wear pattern on the more complex, multicusped teeth are more variable (Figure 53). On some teeth, the pattern of wear reflects an early stage in which usually only the higher main row of cusps are slightly eroded. In other teeth with strongly worn occlusal surface, not only the main row of cusps but the cingulum or in more posteriorly positioned teeth, the secondary row of cusps became also eroded (Figure 53(E)). The last stage of wear on the teeth is a roughly flat dentine surface when the complete tooth crown is eroded. Pits are usually irregular or rarely circular and scratches are mostly robust and V-shaped in cross-section. Scratch orientation slightly varies even among those teeth which are from the same position (Figure 53(B, D)). Some teeth possess short and wide scratches (length $<1 \mathrm{~mm}$ ) without any preferred orientation and here pits are also frequently occur. On the other hand, other teeth (e.g. $15^{\text {th }}$ to $17^{\text {th }}$ in the holotype maxilla, Figure 54) bear moderately or strongly oriented scratches (length $>1-2 \mathrm{~mm}$ ) with a great percent of labiolingual orientation. They are usually parallel or subparallel with each other (see e.g. Fig. 2I, J of Ösi et al. 2007). The wear patterns of the $17^{\text {th }}$ tooth in a subadult specimen, however, differ from those observed on the adult teeth. Its occlusal surface is smoother and the scratches are finer than those of the adult teeth. The eroded last five teeth in the new mandible (MTM PAL2012.29.1.) show that grinding teeth are facing labially being unambiguously the complementary surfaces of the lingually facing wear facets of the upper in situ teeth of the holotype skull. On these lower teeth, the enamel cover is completely missing, thus wear pattern can be detected only on the dentine. The worn dentine surface on these teeth is relatively smooth but it contains large and deep 
areas where the tooth crown became extensively eroded. This is particularly developed on the $15^{\text {th }}$ tooth of a right mandible fragment (MTM PAL2012.30.1.) where the posterior half of this tooth is deeply eroded. On this tooth, some heavy scratches mostly of labiolingual orientation can be observed even with light microscope. The worn, right $15^{\text {th }}$ tooth of the mandible fragment (MTM PAL2012.30.1.) has a smooth dentine surface with a short part of flush EDI on its distal part. This part with the preserved enamel represents the distal, most steeply inclined margin of the deeply concave, eroded area of the tooth crown. On the dentine surface, only a few, small pits can be observed with light microscope so this eroded dentine surface is much smoother than those of neosuchian crocodylifomrs with crushing teeth (e.g. Bernissartia, Unasuchus, Allognathosuchus, Brachychampsa).

Besides the characteristic, nearly labiolingual scratches observable on the teeth of the holotype specimen, a great number of mesiodistally oriented striations also occur on various isolated teeth. These scratches are usually short (length $<1 \mathrm{~mm}$ ) and finer than the longer, transversely oriented scratches, and they are frequent on the worn secondary rows of cusps but also on the mesiodistally extended primary rows.

\section{Adductor muscle reconstruction}

A detailed cranial adductor muscle reconstruction have been fulfilled in Iharkutosuchus by Ösi and Weishampel (2009, Figure 55), so here only a short summary on the estimated mass and architecture of the cranial adductors is provided. In contrast to neosuchians with crushing teeth, the composition of the cranial adductor musculture of Iharkutosuchus was different in many aspects. The lateromedially narrow suborbital fenestrae and palatinal bridge along with the short medial part of the angular indicate that MPTD was not as developed as in Alligator mississippiensis. One of the most significant changes related to jaw adductors of Iharkutosuchus concerns MPTV (Ösi and Weishampel 2009). This muscle has its origin 
surface on the posterior rim and the posterolateral surface of the lateral pterygoid flange (Holliday and Witmer 2007). Unlike in most crocodyliforms, these flanges are strongly reduced in Iharkutosuchus. However, it is suggested that the unusually long posterior pterygoid process could have represented an additional attachment area of MPTV (Figure 55(A)). If this is true, then these posterior bundles of MPTV had a more transverse direction compared with that of Alligator mississippiensis (Busbey 1989) and this could have been important in the motorization of the complex jaw mechanism (see below). The lateral surface of the complete angular of the new mandible (MTM PAL2012.29.1.) possesses a huge insertion surface of MPTV (Figure 55(B)). This outer surface of the bone is generally smooth (not as irregularly ornamented as the anterior lateral surface of the mandible) but is ornamented by very fine, radially oriented grooves. All these features unambiguously indicate a great mass of MPTV relatively comparable to that of Alligator mississippiensis. Another important feature of Iharkutosuchus, previously known only in Hylaeochampsa, is the huge (almost $5 \mathrm{~mm}$ high), triangular protuberance on the ventral surface of the quadrate shaft that served as an extra attachment surface for MAMP. This area is apparently the modification of Iordansky's (1964) crests. This protuberance along with the high postdentary part of the mandible reflects that MAMP could have been an extremely developed adductor muscle in Iharkutosuchus (Figure 55(A)).

In contrast to MPTV and MAMP, the external adductors were strongly reduced in the Hungarian crocodyliform. The supratemporal fenestrae are closed even in juvenile specimens. This indicates that MAMEP could have been a strongly reduced adductor compared to that of extant forms. I cannot find any significant difference between the dorsal part of the laterosphenoid of Iharkutosuchus and that of Alligator mississippiensis that suggests similarity of the relative mass of MPSS between the two taxa. The anterodorsal surface of the surangular, the insertion surface of MAMES and MAMEM, is very short anteroposteriorly 
and not as wide lateromedially as in extant crocodylians (see e.g. Iordansky 1973) but only a medially concave, edge-like margin. On the other hand, a massive coronoid eminence is present behind the last alveolus that is ornamented by a rugose texture medially suggesting attachment area of muscles here. However, this region was not as extended as in extant crocodylians suggesting that MAMES and MAMEM attaching here were less developed than in extant forms.

\section{Jaw mechanism and possible phases of chewing}

The cranial and mandibular morphology, well developed cranial adductor musculature, the presence of complex, multicusped teeth in a strongly heterodont and regionalized tooth row, and the well oriented, extensive wear facets with determined scratch orientation clearly indicate an effective oral food processing with a well controlled dental occlusion and a complex jaw mechanism in Iharkutosuchus. The general cranial and muscular architecture of this crocodyliform strongly resembles those of herbivorous mammals (Ösi and Weishampel 2009) therefore it is likely to have reached the highest efficiency in the processing of food. In herbivorous mammals with a highly positioned craniomandibular joint, $\mathrm{M}$. masseter, which is responsible for slow but active food processing, is the dominant adductor that attaches deep ventrally on the mandible (Maynard and Savage 1959, Smith 1993). Similarly to herbivorous mammals, Iharkutosuchus possesses a dorsoventrally high region of the mandible behind the teeth with a glenoid surface well above the occlusal plane. In addition, MPTV and MAMP responsible for a relatively slow but effective jaw closure were highly developed supporting a relatively long-lasting and balanced but forceful bite to crush and grind its food. On the other hand, the relatively short bundles of the external adductors (MAMES, MAMEM, MAMEP), which are important muscles either in palinal movement (e.g. sphagesaurids) or in fast jaw closure (e.g. in thalattosuchians), are strongly reduced in Iharkutosuchus. In its brevirostine 
skull, the largest multicusped teeth are posteriorly positioned similarly to the bunodont, selenodont or lophodont molars of mammals being as close to the pivot as possible to apply the greatest bite force in crushing/grinding of the food item.

The mandibles of Iharkutosuchus are characterized by a lateromedial movement (Figure 56). Some kind of lateromedial movement has been also reconstructed, for example, in sphagesaurids (Pol 2003, see above), but in these forms no active tooth-tooth contact (i.e. food processing) during side switching was present. However, in Iharkutosuchus, uniquely among crocodyliforms, occlusion of the upper and lower flat molariform teeth occured also during the lateromedial movement of the mandibles (Figure 56). In most crocodyliforms, the lateral pterygoid flanges served to lead and stabilize the mandibles during jaw closure. Furthermore, the quadrate condyles precisely fit into the glenoid surface that is bordered laterally by the posterodorsal part of the surangular. These features are different in Iharkutosuchus. The lateral pterygoid flange is relatively narrower than in other crocodyliforms, thus allowed the mandibles to move in the horizontal plane. The new mandible (MTM PAL2012.29.1.) with its preserved articular clearly supports the earlier hypothesis of Ösi and Weishampel (2009) that the wide glenoid surface (Figure 50(E)) permitted the mandible to move/rotate significantly in the horizontal plane relative to the quadrate condyles. The glenoid surface of Iharkutosuchus is 2-2.5 times longer anteroposteriorly, especially on its lateral side, than the quadrate condyles. Direction of wear striae on the in situ molariform teeth of the holotype skull revealed that the lateromedial mandibular movement was fulfilled by the rotation of the mandibles, similarly to that of some mammals (see Mills 1967), and the center point of rotation has been reconstructed to be within the glenoid surface (Ösi and Weishampel 2009). Until the discovery of the new, complete mandible, it was ambigous whether the two hemi-mandibles were fused or unfused in vivo. The new specimen, in accordance with the earlier specimens with preserved 
mandibular symphysis, further supports the hypothesis that it was unfused in Iharkutosuchus. This unfused nature of the mandibles could have allowed further rotation of the hemimandibles along their long axis to ensure dimensional extension of the movement range. In addition, the short (length $<1 \mathrm{~mm}$ ), mesiodistally oriented striae and the relatively long mandibular glenoid surface indicate that besides lateromedial rotation, the lower jaws performed also some anteroposterior shifting during the powerstroke. Based on the wear striae, this component of movement could not have been as significant as the lateromedial one and its length was far from those described in heterodont notosuchians (Figure 56(G)) but it clearly existed. These features unequivocally indicate that the jaw mechanism of Iharkutosuchus was among the most advanced and complex ones described in any crocodyliforms.

Besides the forceful bite, some jaw muscles of Iharkutosuchus had to motorize the lateromedial movement of the mandibles. In mammals, the reduced pterygoid muscles play an important role in this process. Among the jaw adductors of extant crocodylians, MPTV has the greatest lateromedial component in the line of action (Busbey 1995, Figure 4(D)), so it was concluded that most probably MPTV motorized the lateromedial translation of the mandibles in Iharkutosuchus. Based on the size of the posterior teeth and the position of the lower tooth row relative to the upper one, Ösi and Weishampel (2009) concluded that at least in the posterior part of the tooth row, dental occlusion was persistently bilateral (Figure 56(F)). The more anterior teeth, however, are higher and much smaller (Figure 56(D, E)), and probably moved relatively far from each other during the transverse powerstroke suggesting a rather unilateral occlusion in this region.

Concerning the details of occlusion of the upper and lower teeth, the in situ teeth can help to reconstuct this process. Both in the holotype skull and the newly discovered mandible (MTM PAL2012.29.1.), the posterior five teeth are preserved and they are uniformly eroded, thus no 
difference in the stage of wear can be observed in contrast to that seen in Caiman latirostris (Ösi and Barrett 2011). This shows that occlusion was a precise and well controlled process and wear facets indicate that first of all the central part of the lower teeth occluded with the adjacent, contacting halves of the two opposite upper teeth. Then the lateromedial and the less significant anteroposterior movements during the powerstroke could bring the teeth into a more anterior or posterior position to make food processing more extensive (Figure 56(C, E)). The uniform dental wear on the posterior grinding teeth of Iharkutosuchus suggests that tooth replacement might have been a well controlled process in which not a single tooth but a complete section of the tooth row (e.g. the posterior five grinding teeth of one side) was replaced in one process similarly to that suggested on the basis of tooth wear also in some sphagesaurids (T. Marinho 2010, pers. com.).

Assuming the information described above, the following phases of oral food processing can be reconstructed in Iharkutosuchus:

1) At the beginning of the preparatory stroke the mandibles are in a closed position. During opening of the mouth powered by MDM, the mandibles move simply vertically without any lateromedial movement. During the first few cyles of the preparatory stroke some anteroposterior shifting initiated by pterygoid muscles might existed during jaw closure because the obliquely oriented wear facets on the incisiviform teeth suggest repeated prey capture that might requested precise tooth-tooth contact anteriorly. In various rodents and taeniolabidoid multituberculates, an extensive action of the incisors (i.e. biting) is repeated several times (Weijs 1975, Gorniak 1977, Gambaryan and Kielan-Jaworowska 1995) that results in extensive wear facets on the incisiviforms. In this phase, most probably all jaw adductors acted.

2) During the powerstroke at least two different phases of food processing can be distinguished on the basis of the differentiation of the multicusped teeth (Ösi and Weishampel 
2009). First, a crushing-puncturing phase occured when the anteriorly positioned, smaller and more pointed teeth crushed the food item into smaller pieces (Figure 56(A, B, D)). In this phase most probably the pterygoid muscles and MAMP were the dominant adductors. As the food was triturated, it was transported posteriorly by cyclic movements of the tongue, presumably similarly to that seen in recent forms (Bramble and Wake 1985). This was followed by the action of the larger and flat posterior teeth (Figure $56(\mathrm{C}, \mathrm{F})$ ) where efficient food processing with precise dental occlusion was present. In these last phases, powerstroke became a grinding stroke incorporating progressively more tooth-tooth contact. Lateromedial movement of the mandibles was most significant in this phase that is indicated by the presence of elongated, trasversely oriented scratches on the posterior teeth. Food was completely ground up by the flat, posterior molariform teeth making it ready to swallow. Of the adductor musculature, the well-developed MPT and MAMP played an important role in the lateromedial movement of the jaws and thereby in the processing of the food.

Acynodon Buscalioni, Ortega and Vasse, 1997

(Figure 57, 58)

Holotype-Left maxilla (MCNA 7497).

Referred specimens-Acynodon iberoccitanus: complete skull (ACAP-FX1), an almost complete skull with premaxillary and maxillary dentition (ACAPFX2), posterior skull fragments (ACAP-QR1, ACAP-M1343), left ramus of a mandible (ACAP-M260), right dentary (ACAP-QR7). Acynodon adriaticus: skull with lower jaws and the anterior half of the postcranium (MCSNT 57248), dorsal ribs and osteoderms MCSNT 57032.

Type Locality_Laño, North Central Spain (Buscalioni et al. 1997).

Temporal range- Santonian to Maastrichtian. 
Remarks - Altogether three valid species of Acynodon have been erected. The type species, Acynodon iberoccitanus (Buscalioni et al. 1997) was described from various localities of the Late Cretaceous of Spain and France (Martin and Buffetaut 2005, Martin 2007). Acynodon lopezi has been erected on the basis of isolated teeth (Buscalioni et al. 1997). Acynodon adriaticus (Delfino et al. 2008) was described from the Santonian of Italy. In addition, fragmentary material rerferred to Acynodon was described from other localities of Spain (López-Martínez et al. 2001) and Romania (Martin et al. 2006).

Among the three species of Acynodon, Acynodon iberoccitanus and Acynodon adriaticus are of great importance because they have well preserved cranial and dental material. The skulls and mandibles of these two species are similar in various aspects, thus most parts of the analysis presented below refers to Acynodon in general. However, in some cases, especially in the case of the dentition, some important differences can be detected that are certainly related to the different feeding habit of these species, so they are discussed here comparatively in more detail.

\section{General cranial and mandibular morphology}

Acynodon was a small-bodied animal (max. body length approximately $1-1.5 \mathrm{~m}$ ) with a flat and bevirostrine skull. The lateromedially wide and confluent external nares are facing dorsally. Compared to heterodont notosuchians, the orbits are smaller relative to skull length, oval shaped, and facing dorsally, slightly laterodorsally. The skull is wide and massive posteriorly as can be well observed in ACAPFX2. Compared to skull length, medium-sized anteroposteriorly elongated supratemporal fenestrae are present. Whereas in Acynodon iberoccitanus they occupy approximately $20-25 \%$ of the total skull table (Figure $57(\mathrm{~A})$ ), in Acynodon adriaticus they are slightly smaller, being approximately $15-20 \%$ of the total skull table. 
Lateral temporal fenestrae are small to medium-sized, triangular in shape and relatively anteriorly positioned. Although recently it was suggested that Acynodon is a member of the family Hylaeochampsidae (Rabi and Ösi 2010, Turner and Brochu 2010) and thus it is closely related to the other peculiar hylaeochampsid Iharkutosuchus, a number of differences of cranial and dental features can be recognized between the two genera. Whereas Iharkutosuchus has a narrow rostrum with concave lateral margin of the skull, Acynodon is more broad-snouted, similarly to various alligatoroids. Some minor differences between the two Acynodon species can also be observed that perhaps were related to the different life style of the two species. In dorsal view, A. iberoccitanus has a completely straight lateral skull margin and a broad tip of the snout in contrast to that of $A$. adriaticus where it is slightly convex laterally and anteriorly it is more pointed (Delfino et al. 2008). The quadrate is broad and short in both species, but it is slightly longer posteriorly than that of Iharkutosuchus. It is posteroventrally oriented (but not as much as in Iharkutosuchus) and the lateral and medial quadrate condyles are separated by a shallow intercondylar groove. As in Iharkutosuchus, the medial condyle protrudes more posteroventrally than the lateral one. Whereas the ventral surface of the quadrate shaft of Iharkutosuchus bears a huge, triangular protuberance for the attachment of MAMP, Acynodon possesses a promient, anteromedial-posterolaterally oriented crest seen on the smaller specimen (ACAP-FX1, Figure 57(B)). This crest corresponds to the muscle scar 'A' of Iordansky (1964). On the slightly larger specimen (ACAP-FX2), this ridge is more robust towards the condylar end of the quadrate. In addition, medial to muscle scar 'A', another, shorter one (muscle scar 'B' of Iordansky 1964) is also present that is almost mediolaterally oriented. Uniquely, a hook-shaped process is present on the medial side of the medial quadrate condyle and of the retroarticular process on both sides of the skull in Acynodon adriaticus (Delfino et al. 2008). In posterior view, the skull of Acynodon is quite similar to that of Iharkutosuchus, although the supraoccipital is not part of 
the skull roof, in contrast to the latter genus. Based on Martin (2007), the occipital condyle preserved on one of the incomplete specimens of Acynodon iberoccitanus (ACAP-FX1) is posteriorly oriented.

The secondary palate of the skull is massive with developed pterygoid-ectopterygoid complex. The maxilla bears a wide and anteroposteriorly elongate, medial shelf that extends from the ectopterygoid to the $11^{\text {th }}$ maxillary alveolus. The suborbital fenestra is large and anteroposteriorly elongated both in Acynodon adriaticus and Acynodon iberoccitanus. Mediolaterally it is much wider than that of Iharkutosuchus. The pterygoid is not complete in any of the specimens of Acynodon iberoccitanus and it is hidden by the mandible in Acynodon adriaticus, but it seems that it has a moderately wide lateral flange (Martin 2007, Figure 57(B)) and at least in Acynodon adriaticus, it possesses an elongate posterior process, similarly to that of Iharkutosuchus. Whereas the choana of Acynodon adriaticus and Iharkutosuchus is a small circular opening within the pterygoid, in Acynodon iberoccitanus it is much wider lateromedially. In Acynodon iberoccitanus, the ectopterygoids are not as wide as in Iharkutosuchus, and it seems that even Acynodon adriaticus has a wider ectopterygoid (Delfino et al. 2008) which feature might be related to its larger teeth (see below). As in other hylaeochampsids, the ectopterygoids form the medial wall of the last two alveoli. In contrast to Iharkutosuchus, Acynodon iberoccitanus does not have an elongated posterior maxillary process. The high number of different features (especially on the posterior dentition) recognized between the two species of Acynodon, and the more similar skull construction of Acynodon adriaticus to that of Iharkutosuchus also support the hypothesis presented by Turner and Brochu (2010) that the two species of Acynodon are not sistertaxa and Acynodon adriaticus represents a new genus.

The mandible of Acynodon iberoccitanus is quite similar to that of Iharkutosuhcus in having a thick mandibular ramus, a relatively short and narrow mandibular symphysis and a closed 
mandibular fenestra (Figure 57(E-G)). The mandibles of Acynodon adriaticus can only be observed in ventral and lateral views and their posterior end from dorsal view. It is not clear if the symphysis of Acynodon was fused or not. In Acynodon iberoccitanus, a nicely preserved, complete, left mandible with preserved symphyseal articular surface indicates that, similarly to that of Iharkutosuchus, the mandibles were not firmly sutured to each other. In $A$.

adriaticus, the two hemimandibles were preserved as connected bones via the ventral margin of the mandibular symphysis, but it seems that they were rotated along their long axis which suggests that there was no strong bony symphyseal contact. In Acynodon iberoccitanus, the posterior end of the splenial forms a massive, wide shelf just medial to the last five dentary alveoli. The dorsal margin of the surangular is an extended $(4 \times 1 \mathrm{~cm})$, slightly concave surface just behind the coronoid process. This is in stark contrast with that of Iharkutosuchus where this part is a very short and edge-like margin. The coronoid process is not as prominent as in Iharkutosuchus. The dorsally and slightly anterodorsally facing glenoid surface is anteroposteriorly short in both species of Acynodon with a shallow intercotylar ridge that fits into the intercondylar groove between the quadrate condyles. The quadrate-mandibular joint is well above the occlusal plane. The retroarticular process is relatively short and as wide as long.

\section{Dentition and possible extant analogues}

The dentition of Acynodon is heterodont without enlarged caniniform tooth (Martin 2007, Delfino et al. 2008), similarly to that of Iharkutosuchus (Ösi 2008) and perhaps Hylaeochampsa (its teeth are unknown). It is important to note that Acynodon adriaticus and Acynodon iberoccitanus have different tooth morphology at least in the upper tooth row that further strengthens the hypothesis that the two species may represent two different genera. 
The five premaxillary teeth and the first ten maxillary teeth of Acynodon iberoccitanus are spatulated and they have approximately the same size (Martin 2007, Figure 58(A, B)). The basal half of the crown of these teeth is circular in cross-section, whereas apically it tends to be flattened ending in a rounded and slightly tapering apical crest. This crest does not bear cusps, denticles or any crennulations but it has a central, mesiodistally oriented groove. The labial side of the crown of these teeth is slightly convex with a few shallow grooves (Martin 2007:fig. 7B). These teeth bear weak, unserrated carinae mesiodistally. Posteriorly, tooth size increases significantly only from the $16^{\text {th }}$ tooth that is approximately 1.5 times larger than the anterior ones. This tooth is followed by the largest tooth in the row, the $17^{\text {th }}$ that is almost two times larger than the anterior teeth. The labial surface of this tooth is ornamented by shallow, vertically oriented enamel ridges. The size of the last, $18^{\text {th }}$ tooth is approximately the same as that of the $16^{\text {th }}$. These posterior teeth have lower and labiolingually wider, bulbous crown compared to the anterior ones but even the largest, penultimate teeth have a small residuum of an apical groove.

The lower dentition of Acynodon iberoccitanus is not as well known as the upper one. Martin (2007) reported that specimen ACAP-M260 preserves the $5^{\text {th }}$ and $7^{\text {th }}$ dentary teeth that are small, bulbous at the base and spatulate at the tip, similarly to that of the upper anterior ones. In another specimen (ACAP-QR7), two complete crowns among the anterior teeth show a spatulated shape, and an unerupted posterior crushing tooth can be also observed (Martin 2007).

The dentition of Acynodon adriaticus is roughly similar to that of Acynodon iberoccitanus, but some important differences can be recognized. The premaxillary and the first 10 maxillary teeth are quite similar in shape and they are peg-like with labiolingually flattened crown (Delfino et al. 2008, Figure 58(G)). The five premaxillary teeth are somewhat larger then the following maxillary teeth but not significantly. These peg-like teeth also possess mesiodistally 
positioned unserrated carinae and they are slightly more pointed than the anterior teeth of Acynodon iberoccitanus. Similarly to Acynodon iberoccitanus, the posterior three teeth of Acynodon adriaticus are enlarged, compared to the anterior ones. Of the 18 upper teeth the $16^{\text {th }}$ are slightly larger than the anterior ones. However, the penultimate tooth is $3-4$ times larger than the anterior teeth in contrast to that of Acynodon iberoccitanus (Figure 58(F)). In addition, the crown morphology appears to be also different. This tooth of Acynodon adriaticus has neither a keel mesiodistally, nor a residuum of an apical groove, and it is longer that tall (Delfino et al. 2008). The labial surface of this tooth is weakly concave and devoid of any ridges or grooves. The lower tooth row is not visible in Acynodon adriaticus.

Concerning recent analogies, the bulbous, posterior crushing teeth of Acynodon adriaticus strongly resemble those of various lepidosaurs (and also crocodylians), thus the recent analogies listed in heterodont neosuchians with crushing teeth can be used in this type of dentition. In the case of Acynodon iberoccitanus, the spatulated anterior teeth, the smaller size of posterior globular teeth relative to those of Acynodon adriaticus, and the presence of a mesiodistally oriented, apical groove distinguish this species from crocodyliforms with typical globidont dentition that may indicate a different, more sophisticated feeding strategy of Acynodon iberoccitanus. In contrast to the simple conical teeth, the spatulate anterior teeth of Acynodon iberoccitanus suggest a more precise and sophisticated prey capture. The anterior, incisiviform teeth of Iharkutosuchus are roughly similar, spatulate teeth, which, however, show a high degree of wear that is probably the result of cutting the food item. Among extant lepidosaurs, various species possess massive, globular crushing teeth in the posterior part of the tooth row (Kosma 2004). These crushing teeth are not completely identical showing some variation regarding their shape, size, ornamentation and apical morphology. Whereas the posterior teeth of the teiid Ameiva plei are smooth and completely globular, the bulbous anterior teeth of Hemisphaeriodon gerrardi are ornamented by fine enamel ridges and 
apically they bear a small, pointed tip or mesiodistally short crest. The largest, penultimate tooth of Hemisphaeriodon is massive and globular, and this tooth is at least three-four times larger than the anterior teeth, similarly to that of Acynodon adriaticus. Both of these extant species are durophagous but Hemisphaeriodon is a specialized molluscivore which preys exclusively on snails and slugs (Kosma 2004). Numerous massive, globular teeth are present in the jaws of various species of Tiliqua. In many cases, especially the anterior teeth bear a pointed tip or a short crest on the occlusal surface of the enamel, as seen in Acynodon iberoccitanus. These forms are, however, not as specialized as Hemisphaeriodon, but they are rather omnivorous consuming snails, insects, small vertebrates, worms, eggs, and fruits (Kosma 2004).

\section{Dental wear}

In Acynodon adriaticus, the lower teeth and the occlusal surface of the posterior upper teeth are hidden, hence their occlusal surfaces cannot be studied. Delfino et al. (2008) described marked wear facets on the anterior peg-like teeth. This might refer to occlusion with the anterior lower teeth. However, the widely spaced configuration and the peg-like crown morphology of these anterior teeth are non-typical for crocodyliforms with well-controlled tooth-tooth contact. Thus I presume that in the case of these teeth, only accidental occlusion accounts for the observed wear facets. Capturing and crushing of hard-shelled food is more conceivable in this case.

Wear pattern on the teeth of Acynodon iberoccitanus are present but very poorly known. First, the teeth usually do not show extensive wear facets and second, a high number of the teeth in the larger skull (ACAP-FX2) of Acynodon iberoccitanus are badly preserved (not complete or covered with thick conservation material), so taking high resolution molds of the teeth was not possible. Martin (2007) reported that the fifth and seventh teeth of the mandible ACAP- 
M260 bear wear facets on the labial surface of the apical region, but no other details on the wear pattern are known. The anterior, spatulate teeth of ACAP-FX2 show no extensive wear on the carinae, only a few apical wear can be observed (Figure 58(B)). The posterior crushing teeth of ACAP-FX2 are mostly complete but the region of the apical crest with the central, mesiodistally oriented groove (Figure $58(\mathrm{C}, \mathrm{D})$ ) show some rough wear pattern that can be observed, for example, on the left $16^{\text {th }}$ and $17^{\text {th }}$ tooth of the upper tooth row. The enamel margin of the apical groove is very irregular indicating the occlusion with hard-shelled prey rather than with the opposite tooth.

\section{Adductor muscle reconstruction}

The morphology of the temporal cavity and mandibular adductor fossa of Acynodon indicates a well-developed jaw adductor musculature without significant specializations in contrast to that reconstructed in Iharkutosuchus. Regarding internal adductors, the wide and posteriorly extended pterygoid flanges along with the high and massive postdentary part of the mandible show that MPTV was well developed, and most probably it was the dominant jaw adductor, similarly to that of extant crocodylians (Schumacher 1973). The relatively large suborbital fenestrae indicate that MPTD was comparable in size to that of extant forms, thus more developed than that of Iharkutoshuchus. MAMP originating from the ventral surface of the quadrate was also at least as robust as in extant crocodyliforms with prominent muscle scar 'A' and 'B' of Iordansky (1964). However, the size of the muscle scars demonstrates that this muscle was not as robust as that of Hylaeochampsa or Iharkutosuchus. MAMES and MAMEM were probably more significant during jaw closure than in Iharkutosuchus because in Acynodon, the dorsal surface of the surangular is much wider and more elongate indicating the attachment of a greater mass of these external adductors. Furthermore, adductors originating around the ledge of the supratemporal fenestra (e.g. MAMEP) were also more 
developed compared to that of Iharkutosuchus because in Acynodon, the supratemporal fenestrae are open and the supratemporal fossa is relatively wide. Martin (2007) reported a small laterosphenoid in Acynodon suggesting a not really important MPSS.

To sum up, relative to rostrum and tooth row length, the jaw adductors of Acynodon could have been well developed and perhaps relatively more powerful than the jaw adductors of extant crocodylians. This robustness of the muscle system apparently served to motorize the crushing function of the enlarged posterior teeth, similarly to other short snouted forms with crushing teeth. However, in spite of the close relationship with Iharkutosuchus, muscle attachment areas do not indicate any specializations, such as significant reduction or developement of a particular group of muscles.

\section{Jaw mechanism and possible phases of chewing}

Martin (2007) and Delfino et al. (2008) suggested some degree of feeding specialization in Acynodon which they based among others on the short and wide rostrum, the heterodont dentition, the absence of enlarged caniniform teeth in the anterior part of the tooth row, and the arrangement of origin and insertion surfaces of jaw adductors. Indeed, Acynodon possesses a great number of cranial and dental specializations compared to the typical crocodylian skull that supports some kind of non-typical feeding preference. However, cranial and dental features rather suggest that jaw mechanism was not as complex as in Iharkutosuchus or in heterodont notosuchians but jaw closure was basically orthal. The study of the jaw joint (Figure 57(G)) indicates that in both species of Acynodon, the quadrate condyles well fitted into the lateral and medial cotyles of the glenoid surface. The glenoid surface is not particularly elongated to allow significant anteroposterior movement of the mandibles. The glenoid surface of Iharkutosuchus is relatively longer and wider than that of Acynodon indicating different jaw actions between the two taxa. Although the symphysis 
of Acynodon adriaticus is preserved as articulated, the hemimandibles are twisted and perhaps they are not firmly sutured, as it is suggested in Acynodon iberoccitanus. Features of the dentition and the occurrence of wear facets are the only indicators of a jaw mechanism that might be different from that of extant, typical crocodylians. It has to be emphasized, however, that the two species of Acynodon show important differences in these aspects that may refer to different feeding strategies and food preference between the two species. The similar sized and spatulated anterior teeth of Acynodon iberoccitanus are more closely spaced than the peglike teeth of Acynodon adriaticus. The posterior bulbous teeth of Acynodon iberoccitanus show some irregular wear on the enamel margin of the apical groove. Wear facets in crocodyliforms with well-controlled dental occlusion, however, usually occur on the lingual side of the upper and the labial side of the lower teeth. These minute wear facets apically are most probably due to the crush of some hard-shelled food rather than direct tooth-tooth occlusion. The labial wear on the fifth and the seventh dentary teeth reported in Acynodon iberoccitanus (Martin 2007) might be the indicators of dental occlusion in this region. However, in the case of a regular and well controlled dental occlusion, a more developed wear pattern on the whole dentition would be expected. Although the occlusal surface of the enlarged, posterior maxillary teeth of Acynodon adriaticus cannot be studied, I suppose that well-controlled dental occlusion did not occur in this species because in lateral view (Delfino et al. 2008:fig. 5c), they appear to be complete and do not show the indication of extensive wear.

Features of the jaw joint and the dentition, and the absence of extensive wear rather indicate a simple orthal mandibular movement in Acynodon. Anteroposterior movement can be easily excluded because of the precise quadrate-articular joint. A lateromedial rotation of the mandibles, similar to that reconstructed in Iharkutosuchus, might have been present which would be supported by the possible unfused mandibular symphysis. However, the lack of 
precise, extensive and labiolingually extended wear facets and the relatively wide pterygoid flanges do not support this idea.

Supposing a simple orthal jaw closure in Acynodon, the phases of oral food processing could be quite similar to neosuchians with posterior crushing teeth. The only thing that certainly made a difference in the prey capture and hold compared to the neosuchians is the absence of enlarged caniniform teeth anteriorly. In this aspect, Acynodon is more similar to Iharkutosuchus (Delfino et al. 2008), thus it can be supposed that similarly to the Hungarian form Acynodon was not an active predator.

\section{The origin and evolution of dental occlusion, jaw mechanism and food processing in}

\section{crocodyliforms}

\section{Functionally heterodont dentition and dental occlusion among archosaurs}

Although the basal tooth construction of archosaurs is the conical tooth morphology with serration (Benton 2004) or unserrated carinae, complex, functionally heterodont dentition sometimes with well-controlled dental occlusion appeared several times convergently in many lineages of the Archosauria. For example, various members of the Stagonolepididae (aetosaurs) show a complex dentition with labiolingually flattened, serrated teeth which, though the dentition is mostly homodont, bear some degree of dental wear (e.g. the anterior maxillary teeth of Stagonolepis, Walker 1961 or different teeth of other members of the clade, Desojo and Báez 2007). Walker (1961:134) suggested that „the crowns of the dentary teeth met the medial ones of the maxillary teeth in a slicing action".

Basalmost pterosaurs, such as Caviramus, Eudimorphodon, Austriadactylus or Carniadactylus, all known from the Late Triassic, possess an extremely heterodont dentition (Wild 1978, Dalla Vecchia et al. 2002, Stecher 2008, Dalla Vecchia 2009). The steeply inclined wear facets on the multicusped teeth of Caviramus and Eudimorphodon 
unambiguously demonstrate dental occlusion and efficient oral food processing at least in these basalmost forms. Interestingly, these adaptations to oral food processing never appeared again during the following 140 million years of evolutionary history of pterosaurs (Ösi 2011). Because the origin and phylogenetic position of the Pterosauria is not clear (see Bennett 2012, Prondvai and Ösi 2011 for a review) and the currently known oldest and basalmost pterosaurs are these strongly specialized, heterodont forms, it is not evident what kind of dentition existed in the ancestral 'protopterosaurs'. In other words, it is ambiguous whether the dentition of the closest ancestors of pterosaurs and of the 'protopterosaurs' was heterodont and they were characterized by an effective oral food processing (i.e. this feature can be regarded as a plesiomorphic charcter for pterosaurs) or these ancestors along with the 'protopterosaurs' had simple conical teeth in a homodont dentition and the first complex dentition occured later.

Regarding dinosaurs, the early evolutionary history of the dentition and oral food processing was similar in some respects to that seen in pterosaurs. Heterodont dentition with cuspidate or denticulate teeth bearing well developed wear facets occur in various lineages (including their basal members) of the group. Whereas in prosauropods well-controlled tooth-tooth occlusion probably did not exist and tooth wear was only ,food-wear facet in the form of a smooth flattish arc" (e.g. in Massospondylus, Plateosaurus; Galton and Upchurch 2004:243), teeth of basal ornithischians (e.g. Lesothosaurus, Pisanosaurus) possess more prominent mesial, distal or occasionally apical wear facets (Norman et al. 2004).

Orthal jaw closure, dental occlusion and effective food processing in basal crocodyliforms On the basis of phylogenetic analyses (see e.g. Clark et al. 2004, Nesbitt 2011 and references therein) it is clear that non-crocodyliform crocodylomorphs (e.g. Sphenosuchus, Junggarsuchus, Kayentasuchus) had a generally homodont dentition composed of conical, 
distally curved, serrated and labiolingually compressed teeth (Walker 1990). Carnivorous predators with roughly similar dentition characteristics occur in the basalmost crocodyliforms, such as Orthosuchus, Hemiprotosuchus, and Protosuchus. This unambiguaously demonstrates that heterodont dentition and its possible functional associates (e.g. dental occlusion, oral food processing, specialized mandibular movement) cannot be regarded as a plesiomorphic charater of the group. On the other hand, complex, specialized dentition also occured among protosuchians. The earliest form with a complex, functionally heterodont dentition is the Kayenta form from the Early Jurassic of North America (Clark 1986, Sues et al. 1994, Figure 59). Dentition of this form provides the earliest evidence for a crocodyliform with tooth wear produced by dental occlusion and effective oral food processing (Ösi 2011). Besides the lower caniniform teeth, bicusped post-caniniform teeth are present that bear extended, approximately horizontally oriented wear facets. Details of the wear facets and the presence of an enlarged lower caniniform in a mandible with strongly fused symphysis indicate that there could not have been significant anteroposterior or lateromedial mandibular shifting but rather an orthal movement was present. In most phylogenetic analyses (e.g. Benton and Clark 1988, Clark 1994, Pol et al. 2004, Pol and Norell 2004a, b, Fiorelli and Calvo 2008, Soto et al. 2011), the Kayenta form is the sistertaxon of the Central Asian Early Cretaceous Edentosuchus (Pol et al. 2004, Pol and Powell 2011) and in some analyses (e.g. Pol et al. 2004) they are the sistergroup of the Protosuchus-Hemiprotosuchus clade. The great similarity between the Kayenta form and Edentosuchus was emphasized in several studies (Clark 1986, Benton and Clark 1988, Sues et al. 1994). The close relationship betwen the two forms is supported among others by the presence of specialized, post-caniniform molariform teeth. However, study of the tooth arrangement, tooth morphology and wear pattern clearly shows a significant difference between the two taxa: whereas the Kayenta form was characterized by well-controlled tooth-tooth contact and thus by marked wear facets on the 
bicusped post-caniniform teeth, in Edentosuchus this mechanism was apparently not present. Based on newly described specimens of the latter form (Pol et al. 2004), the lower, multicusped teeth did not meet with the occlusal surface of the upper teeth but positioned lingual to them when the jaws were in closed position. Nevertheless, some kind of oral food processing could have existed in Edentosuchus that is also suggested by the complex crown morphology and the possible food-wear facets on the dentary teeth of the type material (teeth are completely covered with thick conservation material, so it is hard to identify wear pattern). The small body size (total body length approximately $40-80 \mathrm{~cm}$ ) and the multicusped posterior teeth together with a simple, lower caniniform in the mandible indicate that the Kayenta form and Edentosuchus were rather generalists than specialists. This is also supported by the absence of significant specializations of the cranial adductor muscle and the lack of any kind of specialized jaw mechanism. These two taxa are separated by at least 5060 million years and most probably they are representatives of a more diverse and temporally long-existing (from Early Jurassic to Early Cretaceous) early lineage of specialized crocodyliforms.

Based on the fossil record of non-mesoeucrocodylian crocodyliforms (clades like Gobiosuchus, shartegosaurids and Hsisosuchus, Pol and Norell 2004b, Clark 2011, Figure 59), however, no other species shows this kind of dental specialization among these basal forms. The only exception would be the recently described shartegosaurid, Fruitachampsa callisoni from the Late Jurassic of North America (Clark 2011). This species possesses at least a low degree of heterodonty as that seen in basal notosuchians (araripesuchids, Uruguaysuchus) and in some species of Theriosuchus. Fruitachampsa bears smaller conical teeth anteriorly followed by caniniform teeth (paired in the dentary) still in the anterior half of the tooth row. Posterior teeth have laterally compressed and rectangular crown in lateral aspect that is ornamented by fine vertical ridges extending from the serrated apex on the 
lateral and medial sides half way down the tooth (Clark 2011:fig. 7). The absence of dental wear on these complex teeth and the narrow glenoid surface on the articular indicate that there was no dental occlusion and jaw closure was simply orthal in Fruitachampsa, similarly to most araripesuchids, Uruguaysuchus or Theriosuchus (see below).

\section{Heterodont dentition and the first occurrence of dental occlusion in basal notosuchians}

Among mesoeucrocodylians, heterodont dentition and effective oral food processing occured first among the particularly diverse notosuchians, and this lineage evolved independently from the Kayenta form-Edentosuchus clade of protosuchians (see e.g. Clark 1986, Andrade and Bertini 2008b, O’Connor et al. 2010, Turner and Sertich 2010). From a phylogenetic point of view, among mesoeucrocodylians, the earliest indication of complex, heterodont dentition in some cases with wear facets on the teeth occur in 'araripesuchids' (Figures 59, 60). Although the phylogenetic position and monophyly of 'araripesuchids' are highly debated (see e.g. Ortega et al. 2000, Pol and Apesteguía 2005, Turner 2006, Sereno and Larsson 2009, Martin et al. 2010, Iori and Carvalho 2011, Soto et al. 2011), recent analyses (Sereno and Larsson 2009, Turner and Sertich 2010, O'Connor et al. 2010) suggest a basal position within Notosuchia and a paraphyletic arrangement of the different Araripesuchus species (Figure 60). In general, the teeth of 'araripesuchids' are typical of the heterodont dentition found in most crocodyliforms (simple conical or subconical anterior teeth, a caniniform in the anterior or middle portion of the jaw, and shorter teeth posteriorly). However, the posterior teeth are blunter or labiolingually more flattened than those of extant crocodilians. In Araripesuchus tsangatsangana, these theeth are marked with shallow ridges in the enamel but they neither bear any distinct denticles on the carinae nor wear facets (Turner 2006). In Araripesuchus wegeneri (Buffetaut 1976, Sereno and Larsson 2009:figs 19, $20,21)$, the posterior teeth are finely serrated and they are quite similar to the teeth of 
Uruguaysuchus (Soto et al. 2011) and in some aspects to the posterior, labiolingually compressed, serrated teeth of some Theriosuchus species (e.g. T. sympiestodon Martin et al. 2010). This unambiguously indicates that this type of heterodont dentition, always seen in small-bodied forms (average body length $<1 \mathrm{~m}$ ), convergently evolved in several lineages of crocodyliforms (i.e. among shartegosaurids, notosuchians and neosuchians). In most of these lineages, well-controlled occlusion of the upper and lower teeth providing an effective oral food processing did not occur. However, new and exceptionally preserved specimens of Araripesuchus wegeneri show that at least in this species precise tooth-tooth contact really existed (Sereno and Larsson 2009). The posterior maxillary and dentary teeth bear extensive wear facets and show different stages of abbrasion. Wear facets, if present, always started to occur on the apical region. Due to the mesiodistally elongate, carina-like apex, these wear facets are mesiodistally elongated and narrow. Sereno and Larsson (2009:97) suggested that these facets are the result of ,abrasive wear that has rounded and polished the crown apices” (i.e. they are rather due to tooth-food contact then tooth-tooth occlusion). Besides apical wear, they also reported some steeply inclined, flat worn surfaces, for example, lingually on some maxillary teeth (Sereno and Larsson 2009: fig. 19C) that were interpreted as the result of tooth-tooth occlusion (i.e. in a shearing contact). Comparison of these wear facets with those seen in other heterodont notosuchians clearly supports the hypothesis of Sereno and Larsson (2009). Especially Candidodon shows quite similar wear facets.

Ortega et al. (2000) reported some wear facets on the distal side of the mesiodistally widened, blunt posterior maxillary teeth of Araripesuchus patagonicus. These facets are, however, most probably due to tooth-food contact and not to direct tooth-tooth contact. The hypothesis that dental occlusion and effective oral food processing did not exist in most of these early notosuchians (except for Araripesuchus wegeneri) is further supported by the jaw articulation. Although in Uruguaysuchus and Araripesuchus spp. the quadrate-articular joint is not as 
precise as in modern eusuchians but compared to notosuchians with palinal or proal movement the glenoid surface is still narrower anteroposteriorly. Furthermore, the lateral flanges of the pterygoids are also quite expanded laterally to prevent any lateromedial dislocation of the mandibles. This suggests that jaw closure was simply orthal, even in Araripesuchus wegeneri.

Most recent phylogenetic analyses (Fiorelli and Calvo 2008, Martin et al. 2010, Turner and Sertich 2010, Iori and Carvalho 2011, Soto et al. 2011) put Libycosuchus and Simosuchus (sometimes as sistertaxa of each other) in a basal position close to Uruguaysuchus and Araripesuchus spp. (Figure 60). Unfortunately, the dentition of the enigmatic, North African Libycosuchus is not preserved (Figure 40), so it is unknown whether dental occlusion existed or not and what was the degree of oral food processing, if it was present. However, the highly positioned quadrate-articular joint, being almost identical with that of the precise articulation of modern eusuchians, clearly shows that jaw closure was simply orthal in Libycosuchus. Simosuchus, although it is thought to be a relatively basal form among notosuchians (Turner and Sertich 2010), is among the latest forms (Maastrichtian) with a highly specialized dentition. This unusual dentition described in detail by Kley et al. (2010) clearly demonstrates some kind of specialized feeding habit (most probably herbivory). However, the absence of tooth wear facets and dental occlusion unambiguously demonstrates a relatively primitive condition of oral food processing in this form. In Simosuchus, there was no sophisticated chewing or grinding of the (probably vegetable) food item as, for example, in ornithopod dinosaurs, but the labiolingually compressed, multicusped upper and lower teeth simply provided a shearing mechanism to cut the food as extant herbivorous lizards do today. Accepting the hypothesis that these taxa (Araripesuchus, Uruguaysuchus, Libycosuchus, Simosuchus) are basal members of the Notosuchia, it can be concluded that complex dentition with multicusped teeth appeared already in some of these forms (e.g. Simosuchus), but 
regionalized dentition, well controlled dental occlusion accomplished by a highly complex and sophisticated jaw mechanism occured only in more advanced members of the Notosuchia (Figure 60). These basal forms represent those early lineages of notosuchians where specialization in the feeding habit was most probably not as advanced as in more derived forms. The only exceptions are Araripesuchus wegeneri where precise tooth-tooth contact occured in the posterior segment of the tooth row (Figure 60), and Simosuchus clarki with some kind of specialization to herbivory. However, in both of these species, jaw closure was simply orthal.

\section{Dental occlusion and complex jaw mechanism in advanced notosuchians}

Regarding the phylogenetic context of more derived notosuchians, a great number of recent studies dealed with their systematic relationships and provided sometimes markedly different concepts (Andrade and Bertini 2008b, Fiorelli and Calvo 2008, Novas et al. 2009, Sereno and Larsson 2009, Turner and Sertich 2010, Iori and Carvalho 2011, Soto et al. 2011). For example, whereas in the analysis of Turner and Sertich (2010) Notosuchus is the sistertaxon of Mariliasuchus (Figure 60(D)), O'Connor et al. (2010) put Notosuchus as a basal member of a different clade formed by Simosuchus, Chimaerasuchus+Sphagesaurus and Comahuesuchus+Baurusuchus (Figure 60(B)). In addition, several genera have been documented very recently (e.g. Yacarerani, Armadillosuchus, and Pakasuchus), thus papers published four-five years ago could not include these critical taxa in their analysis. Hence, if we put these data on heterodont dentition, dental occlusion and jaw mechanism into a phylogenetic context, the above mentioned uncertainties and non-included taxa result in different interpretations on the evolutionary history of at least some of these features. Nevertheless, some steps in the evolutionary history of these features do not change even if we apply different phylogenetic interpretations. 
In this work, four different interpretations of the phylogenetic context for Notosuchia (O’Connor et al. 2010, Turner and Sertich 2010, Iori and Carvalho 2011, Soto et al. 2011, Figure 60) have been adopted to demonstrate the possible routes of structural and functional evolutionary changes in the dentition, dental function and jaw mechanism of notosuchians. Accepting the most widely preferred concept that 'araripesuchids' are basal members of the Notosuchia (contra O'Connor et al. 2010), it can be seen that functionally heterodont dentition with complex posterior teeth and possibly some special food preference characterized already the basalmost members of the group ('araripesuchids', Uruguaysuchus, Simosuchus, dentition of Libycosuchus is unknown) and actually occurred in all major clades of the Notosuchia (Figure 60). Among these basal forms, well-controlled dental occlusion with orthal jaw closure occured at least in one lineage (Araripesuchus wegeneri). The most parsimonious opinion is to regard this feature as an autapomorphy of this species and not a synapomorphic charater of the Notosuchia. In three of the phylogenetic concepts listed above (Turner and Sertich 2010, Iori and Carvalho 2011, Soto et al. 2011), Simosuchus or the Simosuchus + Libycosuchus clade are the sistertaxon of all the other, more advanced notosuchians. For an easier identification of these more advanced notosuchians, I introduce the node-based taxon 'Propalinalia' for this clade (see Figure 60, note that in the phylogenetic interpretation of O'Connor et al. [2010] this node cannot be defined). This group contains among others those advanced forms which, besides orthal jaw closure and precise tooth-tooth contact, are characterized by proal (protractive powerstroke) or palinal (retractive powerstroke) movements during the last stages of chewing cycle. It is evident that the basis of all other (proal, palinal, lateromedial) mandibular movement with dental occlusion is orthal movement with dental occlusion. This indicates that the latter feature can be considered as the primitive condition and, following parsimony, a synapomorphy of Propalinalia. 
Tree topology of the four adopted phylogenetic concepts unambiguously indicates that both proal and palinal movements appeared at least two times in the evolution Propalinalia. Among the adopted trees, the concept of Iori and Carvalho (2011) contains the highest number of heterodont taxa (11) characterized by dental occlusion, and their proposed phylogeny shows one of the most simple topographical arrangement of taxa with orthal, proal or palinal jaw movement (Figure 60(A)). Following this topology, two main groups are separated. The first group, composed exclusively of Late Cretaceous forms, is Notosuchus(Comahuesuchus(Mariliasuchus(Yacarerani+Adamantinasuchus))). Notosuchus being the most basal member here, most probably retained the simple orthal movement with dental occlusion. Although jaw articulation, reconstructed adductor muscles, dental features and the presence of wear facets all suggest that a proal movement existed in Notosuchus (Fiorelli and Calvo 2008), I have to agree with Lecuona and Pol (2008) that wear pattern on the teeth did not provide a hard evidence for any significant anteroposterior shifting of the mandibles and those wear facets could have been the result of simple orthal jaw closure. Perhaps, future discoveries will reveal more details of the wear pattern in this form and clarify if proal movement existed or not. If proal movement really existed in Notosuchus, then this lineage would represent the third independent appearance (being the first one in South America) of proal movement within Propalinalia (Figure 60(A)). The dentition of Comahuesuchus is very poorly known but on the basis of an enlarged, serrated lower caniniform (pers. obs. 2008), there was no proal, palinal or lateral movement. The presence of dental occlusion of the postcaniniform teeth within an orthal jaw closure (similar to Candidodon) can be neither supported nor excluded. In all the remaining three genera (Mariliasuchus, Yacarerani, Adamantinasuchus) representing a single clade palinal movement was pointed out, so the retractive powerstroke in the jaw mechanism seems to have appeared once in the ancestor of this clade. 
The second group within Propalinalia is composed of two clades and includes forms with proal as well as palinal movement, and a sub-clade of Baurusuchus ${ }^{+}$'sebecids' which were typical predators with an orthal jaw closure that was not accompanied by dental occlusion (Figure 60(A)). In the work of O'Connor et al. (2010), Comahuesuchus is the sistertaxon of Baurusuchus, and in the topology of Turner and Sertich (2010) they are very closely related to each other (Figure 60(B, D)). In all of the four phylogenetic analyses presented here, these predatory forms are the members of Propalinalia which suggests that dental occlusion and effective oral food processing primarily present in this group disappeared secondarily in this lineage. The sistergroup of these predatory forms is the Chimaerasuchus ${ }^{+}$'sphagesaurids' clade. These taxa are sistertaxa (as Chimaerasuchus+Sphagesaurus) on the trees of the other three studies (O’Connor et al. 2010, Turner and Sertich 2010, Soto et al. 2011) too (Figure 60(B-D)). Chimaerasuchus is from the Early Cretaceous of Asia and characterized by proal jaw movement. On the other hand, 'sphagesaurids' (Sphagesaurus and Armadillosuchus) are from the Late Cretaceous of South America and they certainly exhibited palinal jaw movement, so they are the second lineage which independently evolved retractive powerstroke in the jaw mechanism. It has to be mentioned that in the analysis of Turner and Sertich (2010), Mariliasuchus is the sistertaxon of Notosuchus and thus Mariliasuchus represents a third independent appearance of palinal jaw mechanism in this phylogenetic concept (Figure 60(D)). If we accept the more distant relationship between Notosuchus and Mariliasuchus (see in O'Connor et al. 2010, Iori and Carvalho 2011, Soto et al. 2011) then it shows that during the Early Cretaceous at least two lineages diverged from an ancestral form having orthal jaw closure and dental occlusion: one of them with proal movement reached Asia and the other one with palinal movement existed at least in South America. There is an important difference in the palinal jaw mechanism and dental occlusion of these 'sphagesaurids' (Sphagesaurus and Armadillosuchus) and the earlier mentioned 
Mariliasuchus + Yacarerani+Adamantinasuchus clade: the dental occlusion of the former group was not bilateral but alternating unilateral, so during chewing there was a significant lateromedial component in the mandibular movement (Pol 2003, Marinho and Carvalho 2009). This alternating jaw closure combined with the palinal movement is regarded as one of the most derived, complex and sophisticated jaw mechanisms within Notosuchia. In contrast to that, in the Mariliasuchus+Yacarerani+Adamantinasuchus clade, occlusion was dominantly bilateral (although in Mariliasuchus the presence of a unilateral or occasional unilateral occlusion can be also suggested). In addition, the difference in body size between the two groups with palinal jaw movement is also noteworthy: whereas the "Mariliasuchus clade' has skull size ranging from 6 to $10 \mathrm{~cm}$ and an estimated body length between 40 and 80 cm, sphagesaurids (including Armadillosuchus) are significantly larger with a skull size ranging from 17 to $30 \mathrm{~cm}$ and an estimated body length between 120 and $200 \mathrm{~cm}$. Within the second group of Propalinalia, there is a small clade composed of two Early Cretaceous forms: the African Malawisuchus characterized by proal movement and the South American Candidodon with dental occlusion but probably only orthal jaw closure. This suggests that Candidonon retained the ancestral mechanism of oral food processing but in another lineage represented by Malawisuchus, proal movement appeared. This is the second, independent occurrence of proal movement within Propalinalia (Figure 60(A)). Pakasuchus was only included in the phylogenetic analysis of its initial description (O'Connor et al. 2010). In that topology, Pakasuchus is the sistertaxon of Adamantinasuchus, and they together are the sistergroup of Mariliasuchus. So, it seems that this new, Early Cretaceous African form is the member of one of the clades with palinal movement (Figure 60(B)). In Pakasuchus, having an extreme regionalization in the mammal-like dentition, a well-controlled dental occlusion during jaw closure can be reconstructed (O'Connor et al. 2010). Morphology of the posterior, molariform teeth (orientation of crests and troughs) 
suggests the possibility of some kind of anteroposterior movement (rather palinal than proal) during the powerstroke, but no dental wear pattern is available up to the present to unambiguously support this hypothesis and demonstrate the direction of movement. However, based on the phylogenetic interpretation of O'Connor et al. (2010), the closest relatives of Pakasuchus (i.e. Mariliasuchus and Adamantinasuchus) exhibit palinal movement, thus reconstructing palinal movement also in Pakasuchus is the most parsimonious approach.

Convergent evolution of proal and palinal movements in crocodyliforms and other vertebrates As it was demonstrated above, the current knowledge on the evolutionary history of notosuchians shows that both proal (protraction) and palinal (retraction) jaw movements evolved independently at least two times in these related taxa. This might be strange regarding the relatively few numbers of genera. Furthermore, it could be also confusing that, for example, on the lineage of Chimaerasuchus+'sphagesaurids', Chimaerasuchus is characterized by proal movement and 'sphagesaurids' are characterized by palinal mechanism. However, we have to keep in mind that at present only a couple of dozens of species are referred to Notosuchia; a clade that otherwise existed at least in the last 60 million years of the Cretaceous. It is very probable that this group (and particularly the heterodont forms) was much more diverse (especially in Africa and Asia), and with the knowledge of more and more transitional forms their phylogenetic relationships become also clearer. In the light of a better overview of their phylogeny, the evolutionary pattern of the different complex jaw mechanisms (i.e. proal, palinal, lateromedial) could be also better elucidated. On the other hand, convergent evolution of proal and palinal movements is not confined only to crocodyliforms but occured in a variety of vertebrate groups, sometimes in relatively small clades. For example, one of the earlierst occurrences of palinal mandibular shifting was reported in anomodont synapsids (Kemp 1982, King 1996, Rybczynski and Reisz 2001, 
Angielczyk 2004). Re-evaluation of the anatomy and phylogeny of these Late Permian synapsids (no more than a dozen of taxa within 15 million years) suggested a dual origin of palinal movement within anomodonts. In addition ,the independent evolution of propaliny in several other nonmammalian synapsid clades (e.g., Edaphosauria, Gorgonopsia) suggests that homoplasy may be the rule rather than the exception for this feature" (Angielczyk 2004:268). In rodents, various aspects of the diversified chewing mechanism has been recognized (see e.g. Wood 1965, Weijs 1975, Butler 1985) and a high number of suprageneric taxa are characterized by proal movement. One of these groups is Myodonta that includes the Muroidea and Dipodoidea superfamilies with 1336 and 51 species, respectively (McKenna and Bell 1997). Study of the orientation of microwear scratches on the molars revealed several independent occurrences of proal mandibular movement during powerstroke among the different myodont groups (Charles et al. 2007). On the other hand, a number of myodont clades (as various primitive rodents, Weijs 1994) can be characterized by an oblique pattern (i.e. mesiolingual-distolabial scratches are present on the horizontal wear facet, so the powerstroke was directed anteromedially) and not by a clear anteroposterior shifting of the mandibles. Butler (1985) and Weijs (1994) regarded this anteromedially directed (oblique) powerstroke as the primitive condition. Considering this hypothesis, the phylogenetic context of myodonts based on molecular data (Charles et al. 2007) unambiguously shows that proal jaw movement appeared multiple times among the different myodont clades. In contrast to rodents, various non-mammalian synapsid lineages and allotherian mammals (clades of Haramiyida and Multituberculata) were characterized by retractive powerstroke (i.e. palinal movement) (Crompton 1972, Gingerich 1977, Krause 1982, Gambaryan and Jaworowska 1995, Butler 2000). In these groups, there was either a simple orthal or palinal movement or the combination of these, so the degree of palinal movement was apparently different among the clades. For example, whereas in the theroteinid haramiyids occlusion was 
fully orthal, in haramiyoid haramiyids palinal occlusion evolved to various extents including an extensive palinal movement in Haramiyidae (Butler 2000). This suggests a multiple origin of a clear palinal movement among early mammalian lineages, and the same might have been the case in heterodont notosuchians.

\section{The origin and evolution of crushing dentition}

In the first 70 million years of the evolutionary history of crocodyliforms, bulbous posterior teeth specialized for crushing hard food item apparently did not appear. The earliest evidence for this type of dentition comes from the Early Cretaceous (Barremian) of western Europe. These earliest representatives are Bernissartia and Unasuchus that possess still relatively small, but bulbous crushing teeth posteriorly (Figure 59). (Hylaeochampsa vectiana from the Barremian of the Isle of Wight possesses huge alveoli in the back of the jaws, however, teeth are unknown so it cannot be ruled out that, similarly to its close relative, Iharkutosuchus, this

early form also had a much more complex dentition). The crown surfaces of Bernissartia and Unasuchus, though not as expanded horizontally as in later eusuchians with crushing teeth, already show an extensive occlusal wear with some differences between the two taxa.

Whereas the tooth wear of Bernissartia is generally irregular and no particular orientation of the apical wear facets (e.g. labially or lingually positioned, obliquely oriented) can be observed, Unasuchus shows extended, flat and slightly sloping wear facets. Roughly similar wear facets occur, for example, in Iharkutosuchus. Although the cranial material of Unasuchus is very scant (premaxilla, maxilla, mandible), it is possible that tooth to tooth occlusion was characteristic for this taxon. This is supported by the lateromedially oriented, short but parallel scratches on the obliquely oriented wear facets. If this is the case, then it is the earliest evidence for dental occlusion among neosuchian crocodyliforms and it represents a more advanced lineage of neosuchians than that of Bernissartia. Actually, among a great 
number of cranial features, the position of the choana is also unknown in Unasuchus, so maybe this taxon is more closely related to the basal eusuchian Hylaeochampsa (i.e. a possible hylaeochampsid) than to Bernissartia. In addition, it has the same age (Barreminan) as Hylaeochampsa and probably they were members of the same western European Wealden fauna.

Accepting the hypothesis that Acynodon is a member of Hylaeochampsidae (Rabi and Ösi 2010, Turner and Brochu 2010, Brochu 2011), then Acynodon represents the most basal eusuchian with crushing teeth. Whereas Acynodon iberoccitanus possesses globular but not really enlarged posterior teeth with a groove apically, Acynodon adriaticus have bulbous, rounded crushing teeth posteriorly with the penultimate one being almost three times larger than the anterior teeth. Morphology of the jaw joint shows that jaw movement was simply orthal. Based on tooth morphology and the absence of extensive, oriented wear facets, it has been suggested that regular tooth-tooth occlusion did not exist in any species of Acynodon. Independently from the different concepts on the phylogenetic relationships of Crocodylia (e.g. Brochu 1999, Salisbury et al. 2006, Delfino et. 2008, Buscalioni et al. 2011), posterior crushing teeth appear in various lineages of the group. Among alligatoroids, Brachychampsa, Stangerochampsa and Allognathosuchus are characterized by enlarged bulbous teeth posteriorly and in crocodyloids, Osteolaemus possesses bulbous stocky teeth in the last two alveoli (Buffetaut and Ford 1979).

In all of these forms, jaw mechanism was precise, simple orthal jaw closure and it seems that dental occlusion in the posterior region of the tooth row existed only in Allognathosuchus haupti (the relative size of the posterior crushing teeth is the largest in this species).

Lateromedial jaw movement and the enigmatic Iharkutosuchus 
Lateromedial movement of the mandibles during jaw closure is one of the most complex mechanisms in crocodyliforms that complemented a sophisticated dental occlusion and oral food processing. Based on the architecture of the cranial adductor muscles of extant crocodylians (Iordansky 1964, Schumacher 1973, Busbey 1989, Holliday and Witmer 2007), the orientation of the pterygoid muscles has the most significant lateromedial component, so it is suggested that these muscles could have motorized the side to side movement of the mandibles (Ösi and Weishampel 2009). The capability for a lateromedial movement to accomplish the closure of the mandibles was developed at least twice during the evolution of the Crocodyliformes, however, with different purposes. One of these taxa using lateromedial movement is the sphagesaurids. Here, side to side switching of the mandibles only served to produce an unilateral occlusion of the mandibles (Pol 2003, Marinho and Carvalho 2009), but during the lateromedial movement of the mandibles there was no occlusion and oral food processing. The other clade, Iharkutosuchus was characterized by an efficient lateromedial movement of the mandibles in which the wide and flat, multicusped surfaces of the posterior lower teeth occluded against the upper one and produced almost horizontal, slightly oblique grinding surfaces. Iharkutosuchus is regarded as a member of Hylaeochampsidae (Ösi et al. 2007), a family in which, besides Hylaeochampsa vectiana, recent studies included Acynodon spp. (Rabi és Ösi 2010, Turner és Brochu 2010), Pietraroiasuchus ormezzanoi from the Albian of Italy and Pachycheilosuchus trinquei from the Albian of the Glen Rose Formation (Buscalioni et al. 2011). The mandible and dentition of Hylaeochampsa are unknown and Acynodon, Pietraroiasuchus (Buscalioni et al. 2011), and Pachycheilosuchus (Rogers 2003) were certainly charaterized by a simple, orthal jaw closure. In addition, the latter two taxa did not have a functionally heterodont dentition. This shows that lateromedial jaw movement with simultaneous occlusion reconstructed in Iharkutosuchus was not characteristic for hylaeochampsids but most probably an autapomorphy of the genus. 


\section{Life style of heterodont crocodyliforms on the basis of feeding-related characters}

\section{Terrestrial life style in heterodont protosuchians and notosuchians}

Most of the studies dealing with the anatomical description of heterodont crocodyliforms do not focus on their paleobiological and functional aspects in details. A couple of them (Clark et al. 1989, Wu and Sues 1996, Pol 2003, Andrade and Bertini 2008b, c, Fiorelli and Calvo 2008, Lecuonal and Pol 2008, Nobre et al. 2008, Ösi and Weishampel 2009, Kley et al. 2010) studied some functional aspects of some these forms but almost all of them discuss the feeding mechanism and possible diet of these animals (but see e.g. Pol 2005). Detailed analysis of the life style, feeding preference or habitat of the above discussed heterodont crocodyliforms is beyond the scope of this study, nevertheless, in this chapter I try to collect the most important information that provide insight into the life style of these animals and demonstrate their rather mammalian than typical crocodilian behavioural aspects. This further strengthens the earlier raised hypothesis that the dynamics of crocodyliform and mammalian diversification pattern was markedly different in the Cretaceous of Western Gondwana (i.e. Africa and South America) compared to that of the northern landmasses (see e.g. O'Connor et al. 2010).

First of all, it has to be emphasized that the detailed study of the dental occlusion, jaw mechanism and feeding mechanism of these heterodont crocodyliforms, presented here, revealed that most of these animals could have had a markedly different life style compared to that of typical, modern crocodyliforms. Although we do not have enough evidence (e.g. anatomical, taphonomical, and sedimentological) in the case of every species, currently it is widely accepted that heterodont protosuchians and notosuchians were basically terrestrial animals. Besides the highly specialized Early Jurassic thalattosuchians and perhaps a few 
other basal mesoeucrocodylians, neosuchians were the other main group which perfectly adapted to aquatic habitats. Thus, it is not surprising that, similarly to lizards or land mammals today, a great variety of feeding habits associated with different kinds of efficient oral food processing evolved dominantly among these terrestrial forms, and it is much rarer in aquatic neosuchians (neosuchians with crushing teeth and hylaeochampsids).

Terrestrial lifestyle can be well supported by a high number of cranial (Busbey 1995) and postcranial features and at least some of them can be recognized almost in all heterodont protosuchians and notosuchians. All of these heterodont forms were relatively small-bodied animals with a total body length of $40-120 \mathrm{~cm}$ (see Table 1 ). The only exceptions are sphagesaurids (Sphagesaurus, Armadillosuchus) (estimated total body length between 1.2-2 m) which are slightly larger than other heterodont notosuchians. Their skull is oreinirostral with narrow and high, but not elongate rostrum, the external nares are facing anteriorly and the orbits open laterally, anterolaterally. In many of these forms, the occipital condyle is posteroventrally oriented indicating an erected body posture with anteroventrally facing head. Features of the appendicular skeleton (elongate limb elements, modified articular surfaces on the humeri and femora, construction of the ilium) also support a relatively gracile, cursorial and erected posture in these animals (e.g. Jacobs 1993, Wu and Sues 1996, Pol 2005). Besides osteological features, sedimentological data may also be used as indicators of the mainly terrestrial lifestyle of these animals. Remains of heterodont protosuchians and notosuchians are from fluviolacustrine, fluvial or eolic sequences deposited under hot and highly seasonal, arid to semi-arid climatic conditions. This sedimentological and paleoclimatic interpretation was uniformly concluded for the depositional environments of the Early Jurassic of western North America (Glen Canyon Group, Kayenta Formation, Clark and Fastovsky 1986, Sues et al. 1994), the Early Cretaceous of Central Asia (Tugulu Group, Eberth et al. 2001) and Central East Africa (Galua Formation of Tanzania, O'Connor et al. 
2006, 2010; Dinosaur Beds of Malawi, Gomani 1997), and also for the Late Cretaceous sedimentary basins of South America (Neuquen Basin, Bajo de la Carpa Formation, Leanza et al. 2004; Bauru Basin, Adamantina and Marilia Formations, Fernandes and Coimbra 1996, Candeiro et al. 2006) and Madagascar (Maevarano Formation, Krause et al. 2010).

\section{Burrowing}

In various heterodont notosuchians (e.g. Malawisuchus, Adamantinasuchus, Mariliasuchus, Armadillosuchus, Simosuchus, perhaps Pakasuchus, and Notosuchus) a burrowing life style has been suggested. However, in most cases evidences are indirect (only from anatomy) and the purpose of burrowing is not completely clear: did the animal burrow for underground food resources and/or to create a resort either for itself or multiple individuals to protect themselves from adverse weather, as in extant crocodylians (Campbell 1972)? Was any of these heterodont forms a head-first burrower as limbless amphibians and reptiles today (Gans 1973) and made a construction of underground tunnel system in compact soils (Kley and Kearney 2007) or they simply used their limbs as digging instruments? Detailed study of Simosuchus pointed out that its head skeleton is incosistent with Buckley et al.'s (2000) hypothesis of a head-first burrowing life style (Kley et al. 2010). In Malawisuchus, a half of a dozen, roughly complete, horizontally oriented specimens have been found in one pocket. This and the sediment texture led Jacobs (1993) to suggest that these animals might have been lying in a burrow and had gotten burried. The possibility that this composition represents underground aestivation sites cannot be excluded either. Another example, where sedimentological evidences provide support at least for burrowing lifestyle, is Adamantinasuchus. One of the specimens was found in a hole made in coarse sand beds that was filled later by redish clay and silt. Most probably this animal lived in this burrow that was unexpectedly filled by the fine redish sediment that killed the animal. In these taxa sedimentological data provided 
evidence at least for their living in burrows. However, it is unclear if the burrows were made by these animals or not.

Regarding anatomical evidences of burrowing, one of the best examples for digging behaviour is Mariliasuchus from the Late Cretaceous of South America (Nobre et al. 2008). This animal has a short rostrum, posteroventrally oriented occipital condyle, but most importantly strongly worn anterior teeth. Best seen in specimens MZSP-PV 50 and MN 6756, these procumbent incisiviforms have strongly developed apical wear, but the other surfaces of the tooth crowns are also strongly worn. These apical wear facets are on the labial (outer) surface of both in the case of the upper and lower teeth so they are not the result of toothtooth contact. In some cases, the teeth are completely eroded and the apical wear almost reaches the base of the crown so that the pulp cavity is exposed (e.g. on the left first dentary tooth of MZSP-PV 50). The plane of this apical wear facet is close to vertical, otherwise obliquely positioned relatively to the long axis of the tooth. These features unambiguously indicate that digging with teeth and the rostrum was an important activity in Mariliasuchus. Massive, procumbent teeth with roughly similar, apical wear facet also occur on the teeth of Armadillosuchus. In addition, this form has robust manual claws suitable for digging. Osteological features of Armadillosuchus strongly resemble those of extant armadillos (Marinho and Carvalho 2009): both groups possess heavy but mobile-banded body armor and heavy digging claws on the forefeet (armadillos use their claws for digging and finding food, as well as for making their homes in burrows). All these characters support the hypothesis of Marinho and Carvalho (2009) that Armadillosuchus was a burrowing crocodyliform and used both its teeth and fore limbs for digging. In Mariliasuchus and Armadillosuchus, the purpose of digging could have been first of all to reach the food resource but making their underground homes is also a possible scenario. 


\section{Limits in the reconstruction of dietary regime of heterodont crocodyliforms}

Reconstructing the diet in heterodont crocodyliforms is frequently in the focus of various studies. However, a number of elements of uncertainty prevent a reliable dietary reconstruction in most of these taxa. The first of these elements is the absence of useable wear facets on the enamel. In the case of the absence of gut content, the best method to draw inference on the diet of a fossil animal is the study of wear pattern on the enamel surface (and not on the dentine) and its comparison with those of extant relatives. This method was first developed in mammals (e.g. Walker et al. 1978, Teaford 1988, Ungar 1996). In most of these analyses, extant relatives and their food preference (e.g. in hominids, other primates, see e.g. El-Zaatari et al. 2005) were available to compare enamel wear pattern of the molars. Except for the protosuchian Kayenta form, the hylaeochampsid Iharkutosuchus (Ösi and Weishampel 2009) and the neosuchians with posterior crushing teeth where the occlusal surface of the teeth is approximately horizontal, most heterodont forms possess pointed, conical or labiolingually flattened teeth. Here, the wear facets are mostly on the side or the carinae of the crown and steeply inclined being rather the indicators of tooth-tooth contact (and the direction of jaw mechanism) than that of the quality of processed food. In this case, the contact between the food item and the tooth probably took much shorter than in crocodyliforms with flat, horizontally extended tooth crowns. Nevertheless, details of the wear (e.g. scratch-pit ratio, size, frequency and orientation of scratches, pit size) on the enamel could be informative enough at least to provide a rough estimation for the quality of food (i.e. hard shelled food versus soft food).

The second uncertainty of dietary reconstruction is the seasonal climate in the paleoenvironment. Similar to mammals (Walker et al. 1978) or sauropod dinosaurs of the North American Morrison Formation (Fiorillo 1998), seasonal climate could have caused different composition of microwear sites on the teeth of crocodyliforms too. Alternating wet 
and dry periods appear to be a characteristic feature of the localities of nearly all crocodyliforms that might have forced these animals to have only partially overlapping diets between wet and dry seasons. This could have resulted in different wear patterns even in a single individual.

Third, it was demonstrated in mammals, that wear pattern on the molars usually reports on the feeding history of the last few weeks or days of the animal. This is most probably true of crocodyliforms with very complex dentition, specialized jaw mechanism and relatively fast tooth replacement (e.g. Iharkutosuchus). Thus, the more specimens bearing tooth wear we can study, the better (more realistic) interpretation for the diet we can have.

Fourth, ontogenetic variation in feeding habits is also an important factor, as it is known for example, in Crocodylus johnstoni (Tucker et al. 1996) and in Caiman latirostris, among which the adults of the latter consume snails, fish, birds and mammals, whereas juveniles are particularly insectivorous (Brito et al. 2002). Besides seasonality and ontogenetic variation, some difference within the diet among the individuals of a single species in extant lizards has been also noted (Schwenk 2000). These circumstances indicate that dietary reconstruction in most of these crocodyliforms (as in most fossil vertebrates) is effected by many uncertainties, thus inference for their dietary habits should be drawn with a measure of caution.

\section{Diet in heterodont crocodyliforms}

The diet of the two protosuchians with heterodont dentition appears to be significantly different that is also expected from the presence (in the Kayenta form) and the absence (Edentosuchus) of well controlled and regular dental occlusion. The wear facets on the transversely wide, originally bicusped teeth of the Kayenta form are well developed, close to horizontal but scratches are not long and do not show any preferred orientation. These wear 
facets are, however, not as irregular as that of Caiman latirostris (Ösi and Barrett 2011) but more smooth indicating the consumption of some softer food rather than hard-shelled molluscs. The small size of the skull ( $6 \mathrm{~cm}$ long), the lower caniniform anteriorly, and the bicusped teeth posteriorly suggest the possible consumption of arthropods but the extensive wear facets show that this animal was probably more of a generalist than simply an insectivore. Although Edentosuchus could have had similar body size (body length of approximately $40 \mathrm{~cm}$ ), and in some aspects its dentition also resembles that of the Kayenta form, its posterior teeth are rather stocky, rectangular with more than two shallow cusps (Pol et al. 2004) and not transversely widened and bicuspid as in the Kayenta form. In addition, wear facets are also missing from the teeth of Edentosuchus or at least they are not as prominent as in the North American Kayenta form. Caniniform tooth is also present in Edentosuchus but apperently there was no dental occlusion, so the food processing was certainly not at the same level as that reconstructed in the Kayenta form. Based on the small size, the pesence of a lower caniniform, and the morphology of the posterior teeth, an omnivorous diet (e.g. insects, worms, small vertebrates, carcasses, vegetables) can be reconstructed in Edentosuchus.

Sereno and Larsson (2009) suggested that the taxonomic diversity among the species of Araripesuchus may represent some level of dietary diversification. The slight differences in dental morphology, the presence or absence of denticulate carinae and that of wear facets support this hypothesis. These authors also concluded that Araripesuchus wegeneri could have been a herbivore. The labiolingually flattened teeth with denticulate leaf-shaped or subcircular crowns and the well developed wear facets as the result of regular tooth-tooth occlusion indeed suggest the consumption of some food item that had to be cut and chopped up well before swallowing. However, wear facet in itself is not an authoritative indicator of herbivory. For example, whereas in iguanid lizards with herbivorous diet, tooth-tooth contact 
is not present during chewing (Throckmorton 1976), in the agamid Uromastix aegyptius, wear facets are present that are partially due to precise dental occlusion (Throckmorton 1979). On the other hand, Schwenk (2000:201) noted that herbivory is one of the dietary types that seems ,to promote strong adaptive modification in the teeth. In many herbivorous lizards the teeth are laterally compressed and multicuspate. The number of cusps and therefore the width of the spatulate teeth roughly correspond to the degree of herbivory (Hotton 1955, Montanucci 1968)". No dentition of any species of Araripesuchus shows a typical herbivorous dentition. The dorsoventrally waving tooth row, the presence of some really pointed caniniform-like teeth, and the relatively short section of leaf-shaped or subcircular tooth crowns suggest a wider range of food preference (Figure 61) which, however, in Araripesuchus wegeneri could contain a higher percentage of vegetables compared to other Araripesuchus species. The dentition of Uruguaysuchus is quite similar to that of Araripesuchus wegeneri. One main difference is the absence of wear pattern in the former genus, thus the omnivorous diet suggested by Soto et al (2011) in Uruguaysuchus is acceptable.

Concerning herbivory, in extant reptiles relatively few taxa can be characterized by an obligatory vegetarian diet. These are, for example, tortoises, the green sea turtle (as the only marine herbivorous turtle), various iguanid lizards, some species of teiid (Cnemidophorus spp.) and agamid lizards (Uromastix aegyptius; Throckmorton 1979), and one species of monitor lizards (Varanus olivaceous) (Schwenk 2000, Kosma 2004). Most lizards are euryphagous eating a wide range of food rather than stenophagous consuming one or a few things. This might have been the case in heterodont crocodyliforms too.

Among heterodont notosuchians, Simosuchus could be one of the few specialists with a true adaptation to herbivory (Buckley et al. 2000, Figure 61). Kley et al (2010) have provided a detailed comparison of the teeth of Simosuchus with those of extinct and extant animals 
regarded as herbivores, and they listed five dental charaters among which most of them was already noted in other vertebrate groups by various authors (e.g. Hotton 1955, Montanucci 1968, Barrett 2000, Reisz and Sues 2000). Among these characters, the closely spaced dentition and the slightly oblique ('en echelon') arrangement of the individual teeth are usually not present in heterodont crocodyliforms except for Simosuchus. A facultatively herbivorous diet was noted in Chimaerasuchus from the Early Cretaceous of China (Wu et al. 1995, Wu and Sues 1996, Figure 61). The complex, tritylodontid-like dentition preserved in Chimaerasuchus indeed can be the indicator of the consumption of highly fibrous material; a hypothesis that is further supported by the fine, parallel scratches preserved on the worn enamel of one of the cusps of the most complete maxillary tooth. Nevertheless, the material presently referred to this taxon is very fragmentary to unambiguously demonstrate its herbivorous life style.

Among heterodont notosuchians, the closely spaced, posterior four cheek teeth of Candidodon resemble the teeth of herbivorous reptiles in having labiolingually flattened and denticulate crowns with well developed, serrated cingulum. These teeth with their apical wear facets are strongly reminiscent of those seen in ankylosaurian dinosaurs. However, in Candidodon, this tooth morphology is typical only in the posterior section of the tooth row. Anteriorly, Candidodon possesses large, pointed and serrated caniniforms and conical anterior teeth. Similarly to Araripesuchus wegeneri (Sereno and Larsson 2009), all these dental features suggest a more diverse diet (Figure 61) which perhaps included some vegetarian food. The possible diet of Notosuchus was analyzed recently by some studies. On the basis of the anatomical characteristics of the skull, mandible, and postcranium, Bonaparte (1991) and later Fiorelli and Calvo (2008) suggested a possible herbivore lifestyle for Notosuchus rather than strict carnivore or scavenging habits. On the other hand, Lecuona and Pol (2008) carefully concluded that the difference in wear facets of Notosuchus mostly containing pits and the high 
number of parallel scratches of that seen in Sphagesaurus and Mariliasuchus may indicate difference in their diet too. Indeed, the wear pattern of Notosuchus is different from that found in all other heterodont notosuchians. First of all, because its teeth are covered by very thin enamel layer and some preserved teeth are completely devoid of enamel cover, so in most cases the pits and scratches are on the dentine and not on the enamel in contrast to that seen on the thick enamel cover of Sphagesaurus and Mariliasuchus. Dietary differentiation between Notosuchus and Sphagesaurus and Mariliasuchus may also be supported by the presence of a sophisticated palinal mandibular movement of the latter two taxa. In Notosuchus, if there was any kind of mandibular movement in addition to orthal, then it could have been proal but up to present, wear pattern cannot support this hypothesis. Nevertheless, it is important to note that neither proal nor palinal jaw movement can be regarded as the indicator of herbivory or any other kind of dietary specialization because rodents with proal or oblique mandibular movement consume a great variety of food (from seeds, fruits, arthropods, snails, small vertebrates, carcasses, soils etc).

The dentition of Notosuchus with relatively widely spaced, conical teeth with obliquely positioned, denticulated, single carina can be observed neither in extant herbivorous reptiles nor in extinct forms that suggested to be potential herbivores (e.g. in non-mammalian synapsids, ornithischian dinosaurs, etc.). These dental features along with the heavy wear facets on the dentine in addition to the strongly developed apical wear on some teeth suggest that Notosuchus was probably not a specialist but consumed both softer (plant material) and harder (vertebrates and invertebrates) food.

Similarly to Chimaerasuchus, Malawisuchus is also characterized by proal jaw movement; however dentition of the two taxa was completely different. In contrast to the relatively large, horizontally extended, and multicusped teeth of Chimaerasuchus, the teeth of Malawisuchus are high with a main, pointed cusp surrounded by smaller cusps at the base. This tooth 
morphology in a relatively small, short $(7.6 \mathrm{~cm})$ and high skull strongly resembles the teeth of insectivorous mammals as it was noted by Jacobs (1993). Clark et al. (1989) discussed that the differentiation of the tooth row indicates different function for the teeth: caniniforms for prey capturing and multicusped post-caniniforms for food processing. The relatively weak abrasion of the teeth is comparable with those of some carnivorans. These authors concluded that the complex jaw mechanism inferred from cranial and dental characters suggests more specialized, probably insectivore diet (Figure 61) in contrast to extant crocodylians. There is no doubt at all that among crocodyliforms, Pakasuchus has the most mammal-like dentition that includes massive upper and lower caniniforms, small premolariform teeth, and large, fully complementary upper and lower molariform cheek-teeth with developed wear facets. The caniniforms and the complementary molariform teeth with sharp-edged labial and lingual crests strongly resemble the molars of carnivorous mammals, thus a carnivorous diet is presumable.

In the case of the remaining five studied taxa with complex heterodont dentition (Sphagesaurus, Armadillosuchus, Mariliasuchus, Adamantinasuchus, and Yacarerani), palinal movement has been demonstrated, although they apparently belong to at least two independent lineages of Notosuchia. These five genera represent three main types of dentition: Mariliasuchus has massive, labiolingually slightly flattened, mesiodistally carinated teeth with tuberculated carina and surface; Sphagesaurus and Armadillosuchus possess high, conical teeth with longitudinal enamel wrinkles and a single carina that is obliquely oriented; and Adamantinasuchus and Yacarerani have obliquely facing surfaces with tubercles or cusps arranged in rows. Although jaw mechanism was basically similar, these pronounced differences in the dental features suggest differentiation in their diet too. Among these forms, Mariliasuchus has the less specialized dentition, thus I agree with the conclusion of Andrade and Bertini (2008c) that this animal most probably consumed a variety of hard or fibrous 
items (e.g. coarse leaves, seeds, pinecones, arthropods, small vertebrates). In the contrary, Adamantinasuchus and Yacarerani had an extremly complex tooth morphology and a relatively small, posteriorly positioned oral cavity that allow to infer to a high degree of dietary specialization. Nobre and Carvalho (2006) noted that there is no indication of tooth wear in Adamantinasuchus, and so they supposed a diet different from that of Mariliasuchus with extensive wear. However, I was able to find some wear facets on the central row of cusps on one of the multicusped teeth. In addition, on the basis of skull size and the ossification of skull elements, this specimen of Adamantinasuchus appears to be a juvenile or subadult. Taking the ontogenetic variation in food preference into account, the absence of extensive wear can be explained by the consumption of soft food item (e.g. predominantly insects) in this ontogenetic stage. This food preference might have shifted to other type of food later in adults, as we see in extant crocodylians. Heavy wear facets on the similarly complex, multicusped teeth of the probably adult Yacarerani may support this hypothesis.

Well developed labiolingual and apical wear facets with numerous, parallel striae seen on the teeth of Sphagesaurus and Armadillosuchus indicate regular and sophisticated oral food processing. Wear pattern may suggest the consumption of soft, fibrous food in these taxa but it is evident that the relatively widely-spaced, conical teeth are not the best accessories for a herbivorous diet. In Armadillosuchus, the huge, upper caniniform teeth and the massive, procumbent lower teeth with significant apical and labial wear are certainly related to an underground foraging habit as it was also suggested by Marinho and Carvalho (2009). This feature is not present in Sphagesaurus. Another important difference between Sphagesaurus and Armadillosuchus is their body size. The latter one is 1.5-2 times larger than Sphagesaurus and it is significantly larger than the other heterodont notosuchians. The large size and the armored body might suggest a relatively slow and perhaps herbivorous but nonpredatory animal. However, the formerly demonstrated correlation between body size and diet 
suggested in lizards (e.g. Pough 1973) can no longer be considered as valid because numerous small bodied lizards are mostly or exclusively herbivorous (Schwenk 2000). On the other hand, the relatively heavy and extended body armor unambiguously refers to a less agile animal which collected rather than hunted its prey.

Based on the similar, heavily worn posterior crushing teeth of some specimens of the extant Caiman latirostris (Ösi and Barrett 2011), neosuchian crocodyliforms with crushing teeth most probably had a similar, omnivorous diet which very frequently included durable, hardshelled prey (e.g. molluscs, crustaceans, turtles, Abel 1928, Carpenter and Lindsay 1980). However, the marked difference in wear patterns in other ontogenetically mature specimens of $C$. latirostris indicates that specific, regional differences in food resources might affect the degree and type of dental wear that also can be true in the case of fossil forms.

In the highly specialized hylaeochampsid Iharkutosuchus the arrangement of the dentition, tooth morphology, enamel microstructure, and the microwear patterns suggest that its diet was diverse, including both soft and hard items probably ranging from fibrous plants and fruits, to insects and freshwater invertebrates, or even vertebrate carcasses (Ösi and Weishampel 2009).

\section{Possible dietary niche partitioning and overlap between notosuchians and mammals during the Cretaceous of Western Gondwana}

Heterodont notosuchians and mammals are known among others from the Cretaceous deposits of both Africa and South America. It was often mentioned that the evolution of Mesozoic mammals on these continents was different in several aspects form those of the Northern Hemisphere (Bonaparte 1996, Krause et al. 1997, Pascual and Ortiz-Jaureguizar 2007, Rougier et al. 2011). Whereas Cretaceous mammals in these southern continents were mostly represented by archaic or highly specialized forms, contemporaneous Laurasian mammalian assemblages consist predominantly of multituberculates, metatherians and basal eutherians 
(Kielan Jaworowska et al. 2004). The relative paucity in the Western Gondwanan Cretaceous mammalian fauna was perhaps compensated by peculiar, mammal-like notosuchians that had very similar ecological role to those of mammals in Laurasia (O’Connor et al. 2010). The diversification pattern seen in these crocodyliforms led to the hypothesis suggested by O’Connor et al. (2010:751) that ,notosuchian craniodental novelty probably represents an example of evolutionary-developmental experimentation by a clade in the absence of potentially competitive ecomorphs from other major tetrapod groups (that is, mammals)". However, it was actually never discussed if these Western Gondwanan mammals coexisted with these highly specialized notosuchians or not, and what was the proportional distribution of mammals relative to highly specialized notosuchians in the different Cretaceous assemblages. Furthermore, if they prove to have coexisted, then it is also to be explored whether there was size or dietary niche partitioning to any degree or rather a significant overlap existed in the diet of the representatives of these two clades.

\section{Coexistence of heterodont notosuchians and mammals in the Cretaceous of Western}

\section{Gondwana}

Discoveries of usually small bodied, terrestrial, heterodont notosuchians in Cretaceous sedimentary basins of Gondwanan landmasses revealed a diverse group of highly specialized, terrestrial vertebrates. Except for the Early Cretaceous Central Asian Chimaerasuchus, this specialized group of crocodyliforms occurs only in South America (7 genera), Africa (2 genera) and Madagascar (1 genus). (If the newly described but poorly preserved Labidiosuchus amicum from the Late Cretaceous Marilia Formation [Kellner et al. 2011] is indeed a new, different taxon, than 8 genera can be assigned to the South American branch of heterodont notosuchians). Except for the Malagasy Simosuchus, all these forms (i.e. the Propalinalia) are from Western Gondwana (i.e. South America and Africa) and they are 
characterized by efficient dental occlusion and in most cases with proal or palinal jaw movement.

Both African heterodont notosuchians (Malawisuchus, Pakasuchus) are from the Middle Cretaceous (?Aptian) of Central East Africa and their localities are very close to each other (O’Connor et al. 2010). Although the Cretaceous mammalian record is extremely scanty in Africa (see Krause et al. 2003 for a review), a fragmentary but well preserved mandible assigned to the highly specialized gondwanatherians is known from the Red Sandstone Group that also contained Pakasuchus, and these strata are close to equivalent with the embedding rock (Dinosaur Beds) of Malawisuchus (O’Connor et al. 2010). Although both the heterodont crocodyliform and mammalian record is scanty from the Cretaceous in Africa, there is at least one horizon containing both groups and thereby suggesting their coexistence.

The South American Cretaceous record is much more abundant and diverse in the case of both crocodyliforms and mammals. However, whereas Brazil provided five or six taxa of notosuchians referred to as Propalinalia (Candidodon, Mariliasuchus, Adamantinasuchus, Armadillosuchus, Sphagesaurus including 'Caipirasuchus', and possibly Labidiosuchus), one genus is known from Argentina (Notosuchus) and one from Bolivia (Yacarerani). On the contrary, except for a single lower jaw fragment (Bertini et al. 1993), mammals are completely unknown from the Brazilian Cretaceous but they are relatively frequent especially in Argentina but also in Peru and Bolivia. The reason for this important faunistic difference might be related to both geographical separation and climatic differences between the Brazilian landmass and the western-southwestern regions of the continent. It is supposed that at least during the Campanian-Early Maastrichtian hiatus, a north-eastern Region ("province nord-gondwanienne", i.e. most of Brazil today) and a South-western Region ("province sudgondwanienne" sensu de Broin and de la Fuente 1993) was separated by a seaway (Pascual and Ortiz-Jaureguizar 2007). 
Regarding the Early Cretaceous of South America, Candidodon is the single known heterodont notosuchian characterized by dental occlusion and intraoral food processing. This form is from the Albian Itapecuru Formation that still did not provide any evidence of mammals.

All the other specialized notosuchians from Brazil are form the Upper Cretaceous Adamantina Formation which has a highly debated age (Turonian-Santonian or CampanianMaastrichtian, see details in the analysis of Mariliasuchus). This formation provided the single known Cretaceous mammal specimen form Brazil (Bertini et al. 1993) which means that these highly specialized notosuchians and mammals appear to have coexisted here during the deposition of the Adamantina Formation.

Notosuchus is the single known, heterodont notosuchian from Argentina and it was found in the Santonian aged Bajo de la Carpa Formation. This formation, however, did not contain mammalian remains. On the other hand, the Cenomanian-Turonian Candeleros Formation (Apesteguía et al. 2002, Rougier et al. 2011), especially the Campanian Los Alamitos Formation (Bonaparte 1996, Chornogubsky 2003, Gurovich 2005, Pascual and OrtizJaureguizar 2007), the Campanian Allen Formation (Rougier et al. 2003), the CampanoMaastrichtian La Colonia Formation, the Late Cretaceous Portezuelo Formation (Gurovich 2005), and the Campano-Maastrichtian Rio Colorado Formation provide a rich assemblage of different groups of mammals. Based on this fossil record, it can be concluded that on the Patagonian landmass there is no evidence for the coexistence of highly specialized notosuchians and mammals.

Regarding the question of potential coexistence of heterodont notosuchians and mammals in Bolivia, the situation is much more similar to that found in Patagonia than to that in Brazil because a single taxon (Yacarerani) represents the chewing crocodyliforms but a relatively rich material of mammals (tribosphenic and non-tribosphenic therians, ?dryolestoids indet., 
Gayet et al. 2001; several taxa of marsupials, Marshall et al. 1983, Muizon et al. 1983, Case and Woodburne 1986) have been documented from this region. However, whereas Yacarerani is form the Cajones Formation, the mammals are from the Middle Maastrichtian El Molino Formation. Although the age of the Cajones Formation is not clear (Turonian to Santonian or Maastrichtian, see in the analysis of Yacarerani), the two different stratigraphic units indicate the absence of evidence for the coexistence of heterodont notosuchians and mammals during the Cretaceous of Bolivia.

\section{Size and trophic partitioning}

From a stratigraphic point of view, direct evidence for the coexistence of heterodont notosuchians characterized by highly specialized feeding mechanism and mammals occurs only in the Middle Cretaceous of Central East Africa and in the Late Cretaceous of Brazil. Middle Cretaceous Central East African crocodyliforms are Malawisuchus and Pakasuchus which possess a skull length of $7.6 \mathrm{~cm}$ and $6.1 \mathrm{~cm}$, respectively, and a body length of 50-60 cm. In Malawisuchus, teeth are relatively high and large (mesiodistal width ca. $4 \mathrm{~mm}$ ) with a pointed central cusp and smaller surrounding cusps which features are rather typical for carnivorous/insectivorous diet. A proal mandibular movement was demonstrated in this form (Clark et al. 1989). Pakasuchus has bicrested molariform teeth (mesiodistal width ca. $5 \mathrm{~mm}$ ) divided occlusally by a mesiodistal or slightly labiomesial-linguodistal trough. This tooth morphology resembles the precisely occluding carnassial teeth $\left(\mathrm{P}^{4}-\mathrm{M}_{1}\right)$ of some carnivore mammals. Although an anteroposterior shifting of the mandibles during the powerstroke was suggested (O'Connor et al. 2010) in this form (if there was any then it could have been rather palinal), no detailed information on the wear facets are available to support this hypothesis. The single known mammal fossil, a possible gondwanatherian from the same horizon is known only on the basis of a fragmentary, approximately $2 \mathrm{~cm}$ long mandible with the largest 
$3^{\text {rd }}$ cheek-tooth being the largest $(2.3 \mathrm{~mm}$ mesiodistal length and $1.9 \mathrm{~mm}$ labiolingual width). This suggests a body length of approximately half the size of Pakasuchus. As in other gondwanatherians, the teeth are extremely hypsodont and bear a roughly flat occlusal surface, the morphology of which suggests the occurrence of palinal movement in this form, as it was demonstrated in other gondwanatherians and multituberculates (Krause 1982, Krause et al. 2003, Gurovich 2005). It can be seen, however, that neither the size nor tooth crown morphology are similar between heterodont notosuchians and this possible gondwanatherian. Supposing an adult ontogenetic stage in these specimens, it can be suggested that both size and dietary niche partitioning at least between these heterodont notosuchians and mammals of this region could have been present that might have contributed to the success of these Middle Cretaceous African notosuchians.

In Brazil, a fragmentary lower jaw with a single premolar was found in the Adamantina Formation (Bertini et al. 1993, Candeiro et al. 2006), a stratigraphic unit that also included Adamantinasuchus, Mariliasuchus, Sphagesaurus, and Armadillosuchus (Mariliasuchus is also known from the Marília Formation, Candeiro 2005). The single tooth in this jaw is slightly larger than $1 \mathrm{~mm}$ mesiodistally, so the skull was very small, most probably no longer than $2 \mathrm{~cm}$. The tooth is labioligually flattened, tall and pointed with crests both mesially and distally. These features indicate an insectivorous/carnivorous diet for this small animal (estimated body length is approximately between $12-20 \mathrm{~cm}$ ) and suggest the absence of any kind of anteroposterior mandibular shifting during the powerstroke. Heterodont notosuchians from the Adamantina Formation with an estimated body length between 50-200 cm fall into a much larger size category. In addition, their dental morphology and recognized jaw mechanism also suggest a different, rather omnivorous, facultatively herbivorous diet, so, at least the available material do not show any size or dietary niche overlap between the 
Adamantina notosuchians and mammals. However, we have to keep in mind that the absence of evidences is not the evidence of absence.

Although there is no direct evidence, gondwanatherians might have coexisted and some of them overlapped both in size (5-6 $\mathrm{mm}$ large teeth) and dietary habit (palinal jaw movement, omnivorous/herbivorous diet) with the specialized notosuchians in the Late Cretaceous of South America. Besides gondwanatherians, some dryolestoids also show some overlap in body size. Most mammals (at least 34 different species assigned to eutriconodonts, symmetrodonts, dryolestoids, gondwanatherians, and marsupials) known from the postBarremian Cretaceous deposits of South America, however, represent a different size range with a skull length between 2-6 cm and an estimated body size of 10-60 cm (Bonaparte 1986 a, b, 1996, Jacobs et al. 1988, Chornogubsky 2003, Krause et al. 2003, Gurovich 2005, Pascual and Ortiz-Jaureguizar 2007, Rougier et al. 2011).

Based on teeth, four Late Cretaceous South American mammalian taxa (Mesungulatum houssayi, Paraungulatum rectangulares, Quirogatherium majori, Gondwanatherium patagonicum) overlap in size with those of the smallest heterodont crocodyliforms (e.g Adamantinasuchus, Candidodon). (Quirogatherium might be a junior synonym of Mesungulatum [Pascual and Ortiz-Jaureguizar 2007]). These mammalian forms possess 4 to 6 mm large molars (Bonaparte 1996, Chornogubsky 2003, Gurovich 2005) which are comparable to those of the small- to medium-sized, specialized notosuchians. However, all of these large-sized mammalian species are from the Campanian Los Alamitos Formation of Argentina which did not provide any evidence of heterodont notosuchians. If the above discussed Tanzanian mandible is indeed a gondwanatherian (Krause et al. 2003) and it has a Middle Cretaceous age (as it was demonstrated in the work of O'Connor et al. 2010), then this group should have existed also in South America already from the Middle Cretaceous to the early Paleogene, and perhaps these highly specialized mammals also occured in the Late 
Cretaceous Bauru Basin. Among these relatively large sized mammals, Gondwanatherium has large, hypsodont postcanine teeth with transversal enamel bands and marked, horizontally extended, occlusal wear facets (Bonaparte 1986b, Krause and Bonaparte 1993) the latter of which indicate palinal movement (Gurovich 2005). If gondwanatherians indeed existed in the Bauru Basin (which cannot be supported at present), then they really coud have overlapped with some heterodont notosuchians both in size and dietary habit and could have competed with the latter forms.

Nevertheless, in extant ecosystems, both dietary overlap and partitioning are very frequent both among closely (e.g. sympatric species of a single genus) and distantly related taxa (e.g. lizard versus crocodiles or lizards versus mammals). For example, the coexisting Osteolaemus tetraspis and the Varanus niloticus were studied by Luiselli et al. (1999) in swamp rain forests of south-eastern Nigeria. Osteolaemus was significantly smaller than $V$. niloticus. Both species occupied the same habitat, and their general diet composition was also similar with an overlap of $78.2 \%$. These features suggest that $O$. tetraspis and $V$. niloticus could be potential competitors in the freshwater ecosystems of the Nigerian rainforest. However, interspecific competition was minimal because the main prey type for these species (crabs) is not limited in the environment (Luiselli et al. 1999). Similar dietary overlap (49.5\%-68.7\% in different seasons) of the red fox (Vulpes vulpes) and stone marten (Martes foina) was also documented. Although these species of carnivorous mammals show a marked difference in body size, their dentition and food preference is quite similar (Papakosta et al. 2010). Significant dietary niche overlap between varanid lizards, foxes and cats has been poited out by Sutherland et al. (2011). An interesting example of habitat partitioning has been documented among four extant South American crocodylian species (Caiman crocodylus, Paleosuchus trigonatus, Paleosuchus palpebrosus and Melanosuchus niger; Magnusson 1985). Although these species occur in the same habitats, the four species generally show distinct habitat segregation that 
has significant consequences to the diets of the four species (Magnusson et al. 1987, Farlow and Pianka 2002).

The fossil record of Cretaceous Western Gondwanan heterodont notosuchians and coexisting mammals are, however, too scanty to demonstrate any of these habitat relationships between the different taxa in detail. The only point that certainly can be confirmed is the particular diversity of these highly specialized, mammal-like crocodyliforms which at least in some places of the Cretaceous Western Gondwana strongly influenced the composition of the ecosystem and perhaps also the abundance and diversity of mammals.

\section{Conclusions}

The present study demonstrated that highly specialized, heterodont dentition appeared various times convergently in several lineages of Crocodyliformes. In the heterodont dentition, tooth count is usually reduced and in various taxa tooth row shows a marked regionalization (i.e. presence of incisiviform, caniniform, molariform teeth). It is also clear that basalmost crocodyliforms had a relatively simple, homodont dentition with pointed, conical, slightly curved teeth that can be regarded as the plesiomorhic character of the group. In these basal forms, well-controlled dental occlusion did not exist and jaw closure was simply orthal. The earliest indication of specialized, heterodont dentition can be seen in the Early Jurassic protosuchian Kayenta form. Here tooth-tooth occlusion and efficient oral food processing was present but mandibular movement during the powerstroke was still simply orthal. The Kayenta form indicates that the efficient oral food processing developed already among the earliest crocodyliforms. The Early Cretaceous Edentosuchus, another protosuchian being a very close relative of the Kayenta form also possessed strongly heterodont dentition but the oral food processing powered by a simple orthal jaw closure was not complemented by 
occlusion of the upper and lower teeth which indicates that chewing was not as sophisticated in Edentosuchus as in the Kayenta form.

Along with a highly diverse and complex dentition, complex jaw mechanisms first occured among notosuchians during the Cretaceous, but not among the basalmost forms. Basal forms, such as Araripesuchus spp., Uruguaysuchus, Libycosuchus and Simosuchus have heterodont dentition but, except for Araripesuchus wegeneri, well controlled dental occlusion was not present. The more derived branch of notosuchians was named here as Propalinalia. In Propalinalia, besides orthal jaw closure and precise tooth-tooth contact, various lineages can be characterized by either proal (protractive powerstroke, pterygoid muscles pull the mandible forward) or palinal (retractive powerstroke, external adductors are highly developed and pull the mandible backwards) jaw movement which occured during the last phase of powerstroke. Except for the Chinese Chimaerasuchus, all notosuchians with proal or palinal jaw mechanism are from Western Gondwana. Phylogenetic analyses provided by several authors unambiguously indicate that both proal and palinal movements occured at least two times in the evolution of Propalinalia. Proal jaw movement was characteristic in the Early Creatceous Chimaerasuchus and Malawisuchus, representing two independent lineages evolving this jaw mechanism. Palinal movement can be demonstrated certainly in Late Cretaceous South American forms. These are Mariliasuchus, Adamantinasuchus and Yacarerani on one lineage, and sphagesaurids such as Armadillosuchus and Sphagesaurus on the other one. Sphagesaurids were the most advanced group of Propalinalia in having palinal movement with an alternating jaw closure. This supposes some kind of lateromedial movement of the mandibles but importantly, dental occusion did not occur during side switching. Propalinalia also includes the Early Cretaceous Candidodon and Pakasuchus, and the Late Cretaceous Notosuchus, but apart from precise tooth-tooth occlusion, anteroposterior shifting of the mandibles cannot be supported in any of these taxa. 
Among modern neosuchians, heterodont dentition with posterior crushing teeth developed in various lineages from Early Cretaceous to extant forms. Jaw closure is simply orthal and tooth-tooth occlusion is usually not present in these forms but wear facets are the result of consuming hard food item. The only exceptions appear to have been Unasuchus and Allognathosuchus, where dental occlusion existed (jaw closure was still orthal).

Evidence for a complex jaw mechanism can be found only among the basal eusuchian hylaeochampsids. Iharkutosuchus possesses an extremely heterodont dentition where, at least in the posterior section of the tooth row, bilateral occlusion existed, and a lateromedial rotation of the mandibles was powered by the obliquely oriented pterygoid muscles. In contrast to sphagesaurids, dental occlusion in Iharkutosuchus was present and efficient during the lateromedial movement. Complemented by a relatively fast tooth replacement, this was one of the most sophisticated methods of dental occlusion and intraoral food processing demonstrated so far among crocodyliforms.

Whereas heterodont protosuchians and notosuchians were terrestrial animals, heterodont neosuchians (forms with crushing teeth and Iharkutosuchus) were semi-aquatic forms. All heterodont forms were relatively small bodied animals with a body length between 40 and $200 \mathrm{~cm}$. Based on anatomical and sedimentological-taphonomical features, burrowing in heterodont notosuchians can be supported or at least supposed in various forms (e.g. Malawisuchus, Adamantinasuchus, Mariliasuchus, Armadillosuchus). However, whereas in Mariliasuchus and Armadillosuchus the purpose of digging could have been to reach the food resource, Malawisuchus and Adamantinasuchus probably made their underground homes by digging. Regarding the diet of heterodont crocodyliforms, it is evident that most of the forms were not predators as it is typical in other crocodyliforms but rather consumed and efficiently processed orally a great variety of food. Whereas the small bodied forms with small, pointed teeth (e.g. Kayenta form, Edentosuchus, Malawisuchus) could have been partially 
insectivorous, other forms were probably predominantly omnivorous or alternatively herbivorous. The only member of heterodont notosuchians which could have been mostly herbivorous is Simosuchus. Dietary niche overlap or partitioning most probably existed between heterodont crocodyliforms and mammals but the fossil record is too scant to support this hypothesis. The evolution and diversity of complex jaw mechanisms and efficient oral food processing in crocodyliforms strongly resemble those of the masticatory system of mammals and suggest that the diverse niches filled in predominantly by mammalian groups in North America and Asia were occupied in other Mesozoic ecosystems (e.g. in numerous Gondwanan habitats and on some islands of the Western Tethyan archipelago) by highly specialized crocodyliforms.

\section{Acknowledgements}

I am really grateful to M. Delfino and an anonymous reviewer for their critical comments and suggestions that greatly imporved this manuscript. I am very grateful to E. Prondvai for her critical comments and questions. I thank the following colleagues and institutes to the access the crocodyliform material in their care: I. Souza de Carvalho and T. Marinho (Federal University of Rio de Janeiro, Rio de Janeiro, Brazil); A. Celso, F. Iori and S. Aparecida (Monte Alto Musuem, Sao Paolo State, Brazil); A. Carvalho (University of Sao Paolo, Sao Paolo, Brazil); A. Kellner, D. Dias Rego Henriques, L. Carvalho (National Museum, Rio de Janeiro, Brazil); S. Chapman, P. M. Barrett, C. McCarthy (Natural History Museum, London, U.K.); O. Rauhut (Paläontological Institute, München, Germany); N. Micklich and M. Blume (Darmstadt, Germany); D. Schwarz-Wings (Humboldt-Universität zu Berlin Museum für Naturkunde, Berlin, Germany); X. Xu and J. Li (Institute for Vertebrate Paleontology and Paleoantropology, Beijing, China); P. and A. Mechin (Vitrolles, France); T. Tortosa (Natural History Museum, Aix-en-Provence, France); U. Göhlich and R. Gemel (Natural History 
Museum, Vienna, Austria); S. Apesteguía, P. Gallina, F. Novas (Natural History Museum, Buenos Aires, Argentina); J. Company (Polytechnic University of Valencia, Valencia, Spain). I thank J. Sertich (Denver Musuem of Natural History, USA), P. O’Connor (Ohio University College, Athens, USA), J. Clark (George Washington University, Washington DC, USA), I. Carvalho (UFRJ) Z. Csiki (University of Bucharest), E. Buffetaut (CNRS, Paris, France), E. Frey (Staatliches Museum für Naturkunde, Karlsruhe, Germany), and D. Schwarz-Wings (Humboldt-Universität zu Berlin Museum für Naturkunde, Berlin, Germany), P. M. Barrett (Natural History Museum, London, U.K.), L. Chiappe, (Natural History Museum, Los Angeles, USA), C. Holliday (University of Missouri, Columbia, USA), M. Delfino (University of Torino, Torino, Italy), E. Prondvai, M. Rabi and L. Makádi (MTA-ELTE Lendület Dinosaur Research Group, Budapest, Hungary) for useful consultations, comments and suggestions. I am gratuful to J. Sertich, D. Pol, A.Turner, P. O’Connor, M. Delfino, and F. Novas for providing me photographs on various crocodyliform specimens.

I thank E. Hankó and Zs. Hajdu for digital work on the illustrations. F. Varriale (Johns Hopkins University, Baltimore, USA) showed me preparation of molds taken from the teeth. I am grateful to Á. Görög and K. Bóka (Eötvös University, Budapest, Hungary), K. Buczkó (Hungarian Natural History Museum, Budapest, Hungary), and P. Ozsvárt (MTA-MTM Research Group for Paleontology, Budapest, Hungary) for help with the SEM. I thank P. Ungar (University of Arkansas, USA) for sending us the Microware software.

Financial support is thanked to Bolyai Fellowship, OTKA (PD 73021, NF 84193), MTAELTE Lendület Dinosaur Research Group, Hantken Foundation, The Jurassic Foundation, National Geographic Society, Hungarian Natural History Museum, European Union "Synthesys" Grant. 


\section{References}

Abel O. 1928. Allognathosuchus, ein an die cheloniphage Nahrungsweise angepasster Krokodiltypus des nordamerikanischen Eozäns. Paläont Z. 9:367-374.

Aguilera E, Salas H, Peña E. 1989. La Formación Cajones: Cretácico terminal del subandino central de Bolivia. Rev Técnica YPFB. 10:131-148.

de Andrade MB. 2005. Revisão sistemática e taxonômica dos Notosuchia (Metasuchia, Crocodylomorpha). [Unpublished PhD thesis]. Instituto de Geociências e Ciências Exatas, Universidade Estadual Paulista, Rio Claro. 239p.

de Andrade MB, Edmonds R, Benton, MJ, Schouten R. 2011. A new Berriasian species of Goniopholis (Mesoeucrocodylia, Neosuchia) from England, and a review of the genus. Zool J Linn Soc. 163:S66-S108.

de Andrade MB, Bertini RJ. 2008a. A new Sphagesaurus (Mesoeucrocodylia: Notosuchia) from the Upper Cretaceous of Monte Alto City (Bauru Basin, Brazil), and a revision of the Sphagesauridae. Historical Biology. 20:101-136.

de Andrade MB, Bertini RJ. 2008b. Morphological and anatomical observations about Mariliasuchus amarali and Notosuchus terrestris (Mesoeucrocodylia) and their relationships with other South American notosuchians. Arquiv Mus Nac Rio de Janeiro. $66: 5-62$.

de Andrade MB, Bertini RJ. 2008c. Morphology of the dental carinae in Mariliasuchus amarali (Crocodylomorpha, Notosuchia) and the pattern of tooth serration among basal Mesoeucrocodylia. Arquiv Mus Nac Rio de Janeiro. 66:63-82. 
Andrews CW. 1913. On the skull and part of the skeleton of a crocodile from the Middle Purbeck of Swanage, with the description a new species (Pholidosaurus laevis) and a note on the skull of Hylaeochampsa. Ann Mag Nat Hist. 8(11):485-494.

Angielczyk KD. 2004. Phylogenetic evidence for and implications of a dual origin of propaliny in anomodont therapsids (Synapsida). Paleobiology. 30(2):268-296.

Apesteguía S. 2004. Bonitasaura salgadoi gen. et sp. nov.: a beaked sauropod from the Late Cretaceous of Patagonia. Naturwissenschaften. 91:493-497 .

Apesteguía S, Novas FE. 2003. Large Cretaceous sphenodontian from Patagonia provides insight into lepidosaur evolution in Gondwana. Nature. 425:609-612.

Apesteguía S, Rougier GW, Forasiepi AM, Novas FE, Novacek M. 2002. The mammals of 'La Buitrera', Candeleros Formation (Early Cenomanian, Late Cretaceous), Río Negro Province, Argentina. VIII Congreso Argentino de Paleontología y Bioestratigrafia; 2002; Corrientes, Argentina.

Barrett PM. 2000. Prosauropod dinosaurs and iguanas: speculations on the diets of extinct reptiles. In: Sues H-D, editor. Evolution of herbivory in terrestrial vertebrates: perspectives from the fossil record. New York: Cambridge University Press. p 42-78.

Bennett SC. 2012. The phylogenetic position of the Pterosauria within the Archosauromorpha re-examined. Historical Biology. iFirst article, 2012, 1-19.

Benton MJ. 2004. Origin and relationships of Dinosauria. In: Weishampel DB, Dodson P, Osmolska H, editors. The Dinosauria (second edition). Berkeley: University of California Press. p. 7-20.

Benton MK, Clark JM. 1988. Archosaur phylogeny and the relationships of the Crocodylia. In: Benton MK, editor. The phylogeny and classification of the tetrapods, Vol. 1. London: Clarendon Press. p. 95-338. 
Bertini RJ, Marshall LG, Brito P. 1993. Vertebrate faunas from the Adamantina and Marilia formations (Upper Bauru Group, late Cretaceous, Brazil) in their stratigraphic and paleobiogeographic context. Neues Jahrb Geol P-A. 188:71-101.

Bonaparte JF. 1986a. Sobre Mesungulatum houssayi y nuevos mamíferos cretácicos de Patagonia, Argentina. Actes Congr Argentina Paleontol. 4(2):48-61.

Bonaparte JF. 1986b. A new and unusual Late Cretaceous mammal from Patagonia. J Vertebr Paleontol. 6:264-270.

Bonaparte JF. 1991. Los vertebrados fósiles de la formación Río Colorado, de la ciudad de Neuquén y sus cercanías, Cretácico superior, Argentina. Rev Mus Argent Cienc Nat Bernardino Rivadavia Paleontol. 4:17-123.

Bonaparte JF. 1996. Cretaceous tetrapods of Argentina. Münch Geowiss Abh. 30:73-130 Bramble D. 1974. Occurrence and significance of the Os transiliens in gopher turtles. Copeia. 1974(1):102-109.

Bramble D, Wake DB. 1985. Feeding mechanisms of lower tetrapods. In: Hildebrand M, Bramble D, Liem K, Wake DB editors. Functional Vertebrate Morphology. Cambridge: Harward University Press. p 230-261.

Brito SP, Andrade DV, Abe AS. 2002. Do caimans eat fruit? Herpetol Nat Hist. 9(1):95-96. Brinkmann W. 1992. Die Krokodilier-Fauna aus der Unter-Kreide (Ober-Barremium von Uña (Provinz Cuenca, Spanien). Berl Geowiss Abh (E). 5:1-123.

Brizuela S, Albino AM. 2009. The dentition of the Neotropical lizard genus Teius Merrem 1820 (Squamata Teiidae). Trop Zool. 22:183-193.

Brochu CA. 1999. Phylogenetics, taxonomy, and historical biogeography of Alligatoroidea. J Vertebr Paleontol. 19(6):9-100.

Brochu CA. 2011. Phylogenetic relationships of Necrosuchus ionensis Simpson, 1937 and the early history of caimanines. Zool J Linn Soc. 163:S228-S256. 
de Broin F, de la Fuente MS. 1993. Les tortues fossiles d'Argentine: Synthése. Ann Paléont. 79:169-232.

Brown B. 1934. A change of names. Science. 79(2039):80.

Buckley GA, Brochu CA, Krause DW, Pol D. 2000. A pug-nosed crocodyliform from the Late Cretaceous of Madagascar. Nature. 405:941-944.

Buffetaut E. 1975. Sur l'anatomie et la position systématique de Bernissartia fagesii Dollo, L., 1883, crocodilien du Wealden de Bernissart, Belgique. Bull Inst R Sc N B-S. 51:1-20.

Buffetaut E. 1976. Die biogeographische Geschichte der Krokodilier, mit Beschreibung einer neuen Art, Araripesuchus wegeneri. Sond Geol Rundsch. 70:611-624.

Buffetaut E. 1979. Revision der Crocodylia (Reptilia) aus der Gosau-Schichten (Ober-Kreide) von Österreich. Beitr Paläont Österreich. 6:89-105.

Buffetaut E. 1981. Die biogeographische Geschichte der Krokodilier, mit Beschreibung einer neuen Art, Araripesuchus wegeneri. Geol Rundsch. 70(2):611-624

Buffetaut E. 1982. Radiation évolutive, paléoécologie et biogéographie des Crocodiliens mésosuchienes. Mém Soc Geol France. 142: 1-88.

Buffetaut E, Ford RLE. 1979. The crocodilian Bernissartia in the Wealden of the Isle of Wight. Palaeontology. 22(4):905-912.

Busbey AB. 1982. Form and function of the jaw musculature of Alligator mississippiensis. [PhD dissertation]. [Chicago (IL)]: University of Chicago.

Busbey AB. 1989. Form and function of the feeding apparatus of Alligator mississippiensis. J Morphol. 202:99-127.

Busbey AB. 1995. The structural consequences of skull flattening in crocodilians. In: Thomson JJ editor. Functional morphology in vertebrate paleontology. Cambridge: Cambridge University Press. p. 173-192. 
Buscalioni AD, Sanz JL. 1990. The small crocodile Bernissartia fagesii from the Lower Cretaceous of Galve (Teruel, Spain). Bull Inst R Sc N B-S. 60:129-150.

Buscalioni AD, Ortega FL, Vasse D. 1997. New crocodiles (Eusuchia: Alligatoroidea) from the Upper Cretaceous of southern Europe. CR Hebd Acad Sci IIA. 325:525-530.

Buscalioni AD, Piras P, Vullo R, Signore M, Barbera C. 2011. Early usuchia crocodylomorpha from the vertebrate-rich Plattenkalk of Pietraroia (Lower Albian, southern Apennines, Italy). Zool J Linn Soc-Lond. 163:199-227.

Butler PM. 1985. Homologies of molar cusps and crests, and their bearing on assessments of rodent phylogeny. In: Luckett WP and Hartenberger J-L, editors. Evolutionary relationships among rodents—a multidisciplinary analysis. New York: Plenum. p 381401.

Butler PM. 2000. Review of the early allotherian mammals. Acta Palaeontol Pol. 45(4):317342.

Campbell HW. 1972. Ecological or physiological interpretations of crocodilian nesting habits. Nature. 238:404-405.

Candeiro CRA. 2005. Geologia e Paleontologia de vertebrados da Formação Marília (Neomaastrichtiano) no sítio paleontológico de Peirópolis. Caminh Geog. 11(16):117124.

Candeiro CRA, Santos AR, Rich TH, Marinho TS, Oliveira EC. 2006. Vertebrate fossils from the Adamantina Formation (Late Cretaceous), Prata paleontological district, Minas Gerais state, Brazil. Géobios. 39:319-327.

Carpenter K, Lindsey D. 1980. The dentary of Brachychampsa montana Gilmore (Alligatorinae; Crocodylidae), a Late Cretaceous turtle-eating alligator. J Paleontol. $54: 1213-1217$. 
Carvalho IS. 1994. Candidodon: um crocodilo com heterodontia (Notosuchia, Crétáceo Inferior-Brasil). Anais da Academia Brasiliera de Ciencias. 66:331-346.

Carvalho IS, Bertini RJ. 1999. Mariliasuchus, um novo Crocodylomorpha (Notosuchia) do Cretáceo da Bacia Bauru, Brasil. Revista Geologia Colombiana. 24:83-105.

Carvalho IS, Campos DA. 1988. Um mamífero triconodonte do Cretáceo Inferior do Maranhão, Brasil. An Acad Bras Cienc. 60:437-446.

Carvalho IS, Vasconcellos FM, Tavares SAS. 2007. Montealtosuchus arrudacamposi, a new peirosaurid crocodile (Mesoeucrocodylia) from the Late Cretaceous Adamantina Formation of Brazil. Zootaxa. 1607:35-46.

Case JA, Woodburne MO. 1986. South American marsupials: a successful crossing of the. Cretaceous-Tertiary boundary. Palaios 1:413-416.

Charles C, Jaeger JJ, Michaux J, Viriot L. 2007. Dental microwear in relation to changes in the direction of mastication during the evolution of Myodonta (Rodentia, Mammalia). Naturwissenschaften. 94:71-75.

Chornogubsky CL. 2003. Revisión preliminar de los mamíferos de la Formación Los Alamitos (Campaniano Maastrichtiano, provincia de Río Negro, Argentina) [PhD dissertation]. [Buenos Aires, Argentina]: Universidad de Buenos Aires.

Clark JM. 1986. Phylogenetic relationships of the crocodylomorph archosaurs [Ph.D. dissertation]. [Chicago (IL)]: The University of Chicago.

Clark JM. 1994. Patterns of evolution in Mesozoic Crocodyliformes. In: Fraser NC, Sues HD, editors. In the shadow of the dinosaurs. New York: Cambridge University Press. p. 84 97.

Clark JM. 2011. A new shartegosuchid crocodyliform from the Upper Jurassic Morrison Formation of western Colorado. Zool J Linn Soc-Lond. 163:152-172. 
Clark JM, Fastovsky DE. 1986. Vertebrate biostratigraphy of the Glen Canyon Group in northern Arizona. In: Padian K, editor. The beginning of the age of dinosaurs: vertebrate faunas across the Triassic-Jurassic boundary. New York: Cambridge University Press. p. $285-301$.

Clark JM, Jacobs L, Downs WR. 1989. Mammal-like dentition in a Mesozoic crocodylian. Science. 244:1064-1066.

Clark JM, Norell MA. 1992. The Early Cretaceous crocodylomorph Hylaeochampsa vectiana from the Wealden of Isle of Wight. Am Mus Novit. 3032:1-19.

Clark JM, Xu X, Forster CA, Wang Y. 2004. A Middle Jurassic 'sphenosuchian' from China and the origin of the crocodylian skull. Nature. 430:1021-1024.

Cleuren J, De Vree F. 2000. Feeding in crocodiles. In: Schwenk K, editor. Feeding, form, function, and evolution in tetrapod vertebrates. New York: Academic Press. p 337-358.

Colbert E, Mook CC. 1951. The ancestral crocodilian Protosuchus. B Am Mus Nat Hist. 97(3):143-182.

Company J, Pereda-Suberbiola X, Ruiz-Omeňaca JI, Buscalioni AD. 2005. A new species of Doratodon (Crocodyliformes:Ziphosuchia) from the Late Cretaceous of Spain. J Vertebr Paleontol. 25(2):343-353.

Cope ED. 1861. Recent species of Emydosaurian reptiles represented in the Museum of the Academy. Proc Acad Nat Sci Philadelphia. 12:49-551.

Crompton AW. 1972. Postcanine occlusion in cynodonts and tritylodontids. Bull British Mus Nat Hist. Geol. 21(2):27-71.

Crompton AW. 1995. Masticatory function in nonmammalian cynodonts and early mammals. In: Thomason J, editor. Functional morphology in vertebrate paleontology. New York: Cambridge University Press. p. 55-75. 
Crompton A, Hiiemae K. 1970. Molar occlusion and mandibular movements during occlusion, Didelphis marsupialis. L. Zool. J. Linn. Soc. 49:21-47.

Curtis N, Jones MEH, Shi J, O’Higgins P, Evans SE, Fagan MJ. 2011. Functional Relationship between Skull Form and Feeding Mechanics in Sphenodon, and Implications for Diapsid Skull Development. PLoS ONE 6(12): e29804. doi:10.1371/journal.pone.0029804.

Dalla Vecchia FM. 2009. Anatomy and systematics of the pterosaur Carniadactylus nov. g. rosenfeldi (Dalla Vechia, 1995). Riv Italiana Paleontol Strat. 115:159-188.

Dalla Vecchia FM, Wild R, Hopf H, Reitner J. 2002. A crested rhamphorhynchoid pterosaur from the Late Triassic of Austria. J Vertebr Paleontol. 22:196-199.

Daudin FM. 1802. Histoire Naturelle, Générale et Particulière des Reptiles, Vol. 4. F. Dufart, Paris.

Delfino M, Codrea V, Folie A, Dica P, Godefroit P, Smith T 2008. A complete skull of Allodaposuchus precedens Nopcsa, 1928 (Eusuchia) and a reassessment of the morphology of the taxon based on the Romanian remains. J Vert Paleontol. 28(1):111122.

Delfino M, Martin JE, Buffetaut E. 2008. A new species of Acynodon (Crocodylia) from the Upper Cretaceous (Santonian-Campanian) of Villaggio del Pescatore, Italy. Palaeontol. 51(5):1091-1106.

Delfino M, Smith T. 2012. Reappraisal of the morphology and phylogenetic relationships of the alligatoroid Diplocynodon deponiae Frey, Laemmert and Riess 1987, based on a 3Dprepared specimen. J Vert Paleontol, 32(6):1358-1369.

Desojo JB, Báez AM. 2007. Cranial morphology of the Late Triassic South American archosaur Neoaetosauroides engaeus: evidence for aetosaurian diversity. Palaeontology 50(1):267-276. 
Dollo L. 1883. Première note sur les crocodiliens de Bernissart [First note on the crocodilians of Bernissart]. Bull Mus Roy Hist Nat Belg. 2:309-338

van Drongelen W, Dullemeijer P. 1982. The feeding apparatus of Caiman crocodilus: a functional morphological study. Anat Anzeiger. 151:337-366.

Eberth DA, Brinkman DB, Chen P.-J, Yuan F.-T, Wu S.-Z, Li G, Cheng X.-S. 2001. Sequence stratigraphy, paleoclimate patterns, and vertebrate fossil preservation in Jurassic-Cretaceous strata of the Junggar Basin, Xinjiang Autonomous Region, People's Republic of China. Can J Earth Sci. 38:1627-1644.

Endo H, Aoki R, Taru H, Rimura J, Sasaki M, Yamamoto M, Arishima K, Hayashi Y. 2002. Comparative functional morphology of the masticatory apparatus in the long-snouted crocodiles. Anat Histol Embryol. 31(4):206-213.

Erickson BR. 1972. Albertochampsa langstoni, gen. et sp. nov., a new alligator from the Cretaceous of Alberta. Sci Publ Sci Mus Minnesota. 2:1-13.

Estes R. 1969. Relationships of two lizards (Sauria, Teiidae). Breviora. 317: 1-8.

Estes R, Williams EE. 1984. Ontogenetic variation in the molariform teeth of lizards. J Vertebr Paleontol. 4(1):96-107.

Evans AR, Sanson GD. 1998: The effect of tooth shape on the breakdown of insects. J Zool. 246:391-400.

Farlow JO, Pianka ER. 2002. Body size overlap, habitat partitioning and living space requirements of terrestrial vertebrate predators: implications for the paleoecology of large theropod dinosaurs. Hist Biol. 16(1): 21-40.

Fernandes LA, Coimbra AM. 1996. A Bacia Bauru (Cretáceo Superior, Brasil). An Acad Bras Cienc. 68:195-205. 
Fernandes LA, Giannini PCF, Góes AM. 2003. Araçatuba Formation: palustrine deposits from the initial sedimentation phase of the Bauru Basin. An Acad Bras Cienc. 75:173187.

Fiorelli L, Calvo JO. 2008. New remains of Notosuchus terrestris Woodward, 1896 (Crocodyliformes: Mesoeucrocodylia) from Late Cretaceous of Neuquén, Patagonia, Argentina. Arquiv Mus Nac Rio de Janeiro. 66:83-124.

Fiorillo AR. 1998. Dental microwear patterns of the sauropod dinosaurs Camarasaurus and Diplodocus: Evidence for resource partitioning in the Late Jurassic of North America. Hist Biol. 13:1-16.

Fraser NC, Irmis RB, Elliot DK. 2005. A procolophonid (Parareptilia) from the Owl Rock Member, Chinle Formation of Utah, USA. Palaeontol Electr. 8(1):13A.

Galton PM. 1986. Herbivorous adaptations of Late Triassic and Early Jurassic dinosaurs. In: Padian K editor. The Beginning of the Age of the Dinosaurs: Faunal Change across the Triassic-Jurassic Boundary. Cambridge University Press, Cambridge, U.K. p. 203-221. Galton PM, Upchurch P. 2004. Prosauropoda. In: Weishampel DB, Dodson P, Osmolska H, editors. The Dinosauria (second edition). Berkeley: University of California Press. p. 232258.

Gambaryan PP, Kielan-Jaworowska Z. 1995. Masticatory musculature of Asian taeniolabidoid multituberculate mammals. Acta Palaeontol Pol. 40:45-108.

Gans C. 1973. Locomotion and burrowing in limbless vertebrates. Nature. 242:414-415.

Gans F, de Vree G, Gorniak C. 1978. Analysis of Mammalian Masticatory Mechanisms: Progress and Problems. Zbl Vet Med C Anat Histol Embryol. 7:226-244.

Gasparini ZB. 1971. Los Notosuchia del Cretácio de America del sur como un nuevo infraorden de los Mesosuchia (Crocodilia). Ameghiniana. 8:83-103. 
Gasparini ZB, Chiappe LM, Fernandez M. 1991. A new Senonian peirosaurid (Crocodylomorpha) from Argentina and a synopsis of the South American Cretaceous crocodilians. J Vertebr Paleontol. 11(3):316-333.

Gayet M, Marshall LG, Sempere T, Meunier FJ, Capetta H, Rage JC. 2001. Middle Maastrichtian vertebrates (fishes, amphibians, dinosaurs and other reptiles, mammals) from Pajcha Pata (Bolivia). Biostratigraphic, palaeoecologic and palaeobiogeographic implications. Palaeogeo Palaeocl. 169:39-68.

Gilmore CW. 1911. A new fossil alligator from the Hell Creek Beds of Montana. Proc Nat Mus USA. 41(1860):297-302.

Gilmore CW. 1940. New fossil lizards from the Upper Cretaceous of Utah. Smithsonian Misc Coll. 99:1-3.

Gingerich PD. 1977. Patterns of evolution in the mammalian fossil record. In: Hallam A, editor. Patterns of Evolution. Amsterdam: Elsevier Scientific Publishing Co. p. 469-500.

Gomani E. 1997. A crocodyliform from the Early Cretaceous dinosaur beds, northern Malawi. J Vertebr Paleontol. 17:280-294.

Gorniak, G. 1977. Feeding in golden hamsters, Mesocritus auratus. J Morphol. 154:427-458.

Gorniak GC, Rosenberg HI, Gans C. 1982. Mastication in the Tuatara, Sphenodon punctatus (Reptilia: Rhynchocephalia): structure and activity of the motor system. J Morphol. $171: 321-353$.

Gow C. 2000. The skull of Protosuchus haughtoni, an Early Jurassic crocodyliform from southern Africa. J Vertebr Paleontol. 20:49-56.

Greaves WS. 1973. The inference of jaw motion from tooth wear facets. J Paleontol. 47:1000-1001.

Grine F. 1986. Dental evidence for dietary differences in Australopithecus and Paranthropus: a quantitative analysis of permanent molar microwear.J Hum Evol. 15:783-822. 
Gurovich Y. 2005. Bioevolutionary aspects of Mesozoic mammals: description, phylogenetic relationships and evolution of the Gondwanatheria (Late Cretaceous and Paleocene of Gondwana). PhD thesis]. [Buenos Aires, Argentina]: Universidad de Buenos Aires.

Hay OP. 1930. Second Bibliography and Catalogue of the Fossil Vertebrata of North America. Carnegie Institution of Washington. 390(II):1-1074.

Herrel A, Vanhooydonck B,Van Damme R. 2004. Omnivory in lacertid lizards: Adaptive evolution or constraint?. J Evol Biol. 17:974-984.

Hiiemae K, 2000. Feeding in mammals. In: Schwenk K, editor. Feeding: Form, Function and Evolution in Tetrapod Vertebrates. San Diego (CA): Academic Press. p. 411-448.

Holland WJ. 1924. The skull of Diplodocus. Mem Carnegie Mus Nat Hist. 9:279-403.

Holliday C, Witmer LM. 2007. Archosaur adductor chamber evolution: integration of musculoskeletal and topological criteria in jaw muscle homology. J Morphol. 268:457484.

Holliday CM. 2009. New insights into dinosaur jaw muscle anatomy. Anat Rec. 292:12461265.

Holliday CM Witmer LM. 2009. The epipterygoid of crocodyliforms and its significance for the evolution of the orbitotemporal region of eusuchians. J Vert Paleontol. 29(3):715-733.

Hotton N. (1955) A survey of adaptive relationships of dentition to diet in the North American Iguanidae. Am Midi Nat. 53:88-114.

Huxley TH. 1875. On Stagonolepis robertsoni and on the evolution of the Crocodilia. Q J Geol Soc Lond. 31:423-438

Iordansky NN. 1964. The jaw muscles of the crocodiles and some relating structures of the crocodilian skull. Anat Anzeiger. 115:256-280.

Iordansky NN. 1973. The skull of the Crocodilia. In: Gans C, Parson TS, editors. Biology of the Reptilia, Part 4D. New York: Academic Press. p. 201-262. 
Iori F. V. 2008. Um novo Crocodylomorpha Notosuchia Sphagesauridae (Bacia Bauru, Cretáceo Superior) do município de Monte Alto, estado de São Paulo [Thesis]. Instituto de Geociências, Universidade Federal do Rio de Janeiro, 73p.

Iori FV, Carvalho IS. 2009. Morrinhosuchus luziae, um novo Crocodylomorpha Notosuchia da Bacia Bauru, Brasil. Revista Brasileira de Geociências. 39:717-725.

Iori FV, Carvalho IS. 2011. Caipirasuchus paulistanus, a new sphagesaurid (Crocodylomorpha, Mesoeucrocodylia) from the Adamantina Formation (Upper Cretaceous, Turonian-Santonian), Bauru Basin, Brazil. J Vertebr Paleontol. 3186):12551264.

Jacobs LL. 1993. Quest for the African dinosaurs: ancient roots of the modern world. 1st edition. New York: Villard Books. 316 pp.

Jacobs LL, Congleton JD, Brunet M, Dejax J, Flynn LJ, Hell JV, Mouchelin G. 1988. Mammal teeth from the Cretaceous of Africa. Nature. 336:158-160.

Jacobs LL, Winkler DA, Kaufulu ZM, Downs WR. 1990. The dinosaur beds of northern Malawi, Africa. Natl Geogr Res. 6:196-204.

Jones MEH, Curtis N, Fagan MJ, O’Higgins P, Evans SE. 2011. Hard tissue anatomy of the cranial joints in Sphenodon (Rhynchocephalia): sutures, kinesis, and skull mechanics. Palaeontol Electr. 14:17A.

Jones MEH, O’higgins P, Fagan MJ, Evans SE Curtis N. 2012. Shearing Mechanics and the Influence of a Flexible Symphysis During Oral Food Processing in Sphenodon (Lepidosauria: Rhynchocephalia). Anat Rec. 295:1075-1091.

Kälin TA. 1933. Beitrage zur vergleichenden Osteologie des Crocodiliden schadels. Zool Jahrbuch Abt Anat. 57(4):535-714. 
Kellner AWA, Campos DA, Price LI. 1995. New material of Sphagesaurus (Sphagesauridae, Crocodilia) from the Late Cretaceous of Brazil. Atas do XIV Congresso Brasileiro de Paleontologia: 70-71; Uberaba, Brasilia.

Kellner AWA, Figueiredo RG, Azevedo SAK, Campos DA. 2011. A new Cretaceous notosuchian (Mesoeucrocodylia) with bizarre dentition from Brazil. Zool J Linn Soc Lond. 163(1):109-115.

Kemp TS. 1982. Mammal-like reptiles and the origin of mammals. London: Academic Press. $363 \mathrm{pp}$.

Kemp TS. 2005. The origin and evolution of mammals. New York: Oxford University Press. $331 \mathrm{pp}$.

Kielan-Jaworowska Z, Cifelli RL, Luo Z-X. 2004. Mammals from the age of dinosaurs. Columbia University Press, New York. 630 pp.

King G. 1996. Reptiles and Herbivory. London: Chapman and Hall.160 pp.

Kley NJ, Kearney M. 2007. Adaptations for digging and burrowing. In: Hall BK, editor. Fins into Limbs: Evolution, Development, and Transformation. Chicago (IL): University of Chicago Press. p. 284-309.

Kley NJ, Sertich JJW, Turner AH, Krause DW, O’Connor PM, Georgi JA. 2010. Craniofacial morphology of Simosuchus Clarki (Crocodyliformes: Notosuchia) from the Late Cretaceous of Madagascar. J Vertebr Paleontol. 30:1-89.

Knauer J, Siegl-Farkas Á. 1992. Palynostratigraphy of the Senonian formations covering the Late Cretaceous bauxites of the Bakony mountains. A Magyar Állami Földtani Intézet Évi Jelentése az 1990-es Évröl. p. 463-471. [In Hungarian].

Kosma R. 2004 The dentitions of recent and fossil Scincomorphan lizards (Lacertilia, Squamata) - systematics, functional morphology, Palaeoecology [Unpublished $\mathrm{PhD}$ thesis] Hannover. 187 pp. 
Krause DW. 1982. Jaw movement, dental function and diet in the Paleocene multituberculate Ptilodus. Paleobiology. 8(3):265-281.

Krause DW, Bonaparte JF. 1993. Superfamily Gondwanatherioidea: a previously unrecognised radiation of multituberculate mammals in South America. P Natl Acad Sci USA. 90:9379-9383.

Krause DW, Prasad GVR, Koenigswald Wv, Sahni A, Grine FE (1997) Cosmopolitanism among Gondwanan Late Cretaceous mammals. Nature. 390:504-507

Krause DW, Gottfried MD, O’Connor PM., Roberts EM. 2003. A Cretaceous mammal from Tanzania. Acta Palaeontol Pol. 48(3): 321-330.

Krause DW, Sertich JJW, Rogers RR, Rasoamiaramanana AH, Kast SC, Buckley G. 2010. Overview of the discovery, distribution, and geological context of Simosuchus clarki (Crocodyliformes: Notosuchia) from the Late Cretaceous of Madagascar. In: Krause DW, Kley NJ, editors. Simosuchus clarki (Crocodyliformes: Notosuchia) from the Late Cretaceous of Madagascar. J Vertebr Paleont 30(Supplement 1): 4-12.

Kuhn O. 1968. Die Vortzeitlichen Krokodile. Krailing,München: Verlag Oeben. 124 pp. Leanza HA, Apesteguía S, Novas FE, Fuente MS. 2004. Cretaceous terrestrial beds from the Neuquén Basin (Argentina) and their tetrapod assemblages. Cretaceous Res. 25:61-87. Lecuona A, Pol D. 2008. Tooth morphology of Notosuchus terrestris (Notosuchia:

Mesoeucrocodylia): new evidence and implications. Comptes Rendus Palevol. 7:407-417.

Li J. 1985. A revision of Edentosuchus tienshanensis Young from the Tugulu Group of Xingjiang Autonomous Region. Vertebrat Palasiatic. 23:196-206.

Lieberman DE, Crompton AW. 2000. Why fuse the mandibular symphysis? A comparative analysis. Am J Phys Anthropol. 112: 517-540.

López RM. 1975. Informe estratigráfico de los ríos Alto Moile, Alto Isarsama, Ichoa, Alto Beni y Tequeje. Informe YPFB GXG-2600. 
López-Martínez N, Canudo JI, Ardévol L, Pereda Suberbiola X, Orue-Extebarria X, CunecaBescó SG, Ruiz-Omenaca JI, Murelaga, X, Feist M. 2001. New dinosaur sites correlated with Upper Maastrichtian pelagic deposits in the Spanish Pyrenees: implications for the dinosaur extinction pattern in Europe. Cretaceous Res. 22:41-61.

Lucas SG, Estep JW. 2000. Osteology of Allognathosuchus mooki Simpson, a Paleocene crocodilian from the San Juan Basin, New Mexico, and the monophyly of Allognathosuchus. In: Lucas SG, editor. New Mexico’s Fossil Record 2, New Mexico Mus Nat Hist Sci Bull 16:155-168

Luiselli L, Aakani GC, Capizzi D. 1999. Is there any interspecific competition between dwarf crocodiles (Osteolaemus tetraspis) and Nile monitors (Varanus niloticus ornatus) in the swamps of central Africa? A study from south-eastern Nigeria. J Zool Lond. 247:127131.

Magnusson WE. 1985. Habitat selection, parasites and injuries in Amazonian crocodilians, Amazoniana 9:192-204.

Magnusson WE, Viera da Silva E, Lima AP. 1987. Diets of Amazonian crocodilians, J Herpetol. 21:85-95.

Marshall G, Muizon CH, Sigé B. 1983. Late Cretaceous Mammals (Marsupialia) from Bolivia. Géobios. 16:739-745.

Marinho TS, Carvalho IS. 2009. An armadillolike sphagesaurid crocodylifom from the Late Cretaceous of Brazil. J S Am Earth Sci. 27:36-41.

Martin JE. 2007. New material of the Late Cretaceous globidontan Acynodon iberoccitanus (Crocodylia) from southern France. J Vertebrate Paleontol. 27(2):362-372.

Martin JE, Buffetaut E. 2005. An overview of the Late Cretaceous crocodilian assemblage from Cruzy, southern France. Kaupia. 14:33-40. 
Martin J, Csiki Z, Grigorescu D, Buffetaut E. 2006. Late Cretaceous crocodilian diversity in Haţeg Basin, Romania. Hantkeniana. 5:31-37.

Martin JE, Rabi M, Csiki Z. 2010. Survival of Theriosuchus (Mesoeucrocodylia: Atoposauridae) in a Late Cretaceous archipelago: a new species from the Maastrichtian of Romania. Naturwissenschaften. 97:845-854

Maynard Smith J, Savage RJG. 1959. The mechanics of mammalian jaws. School Sci Rev. 40:289-301.

McKenna MC, Bell SK. 1997. Classification of Mammals Above the Species Level. New York: Columbia University Press. 631 pp.

Meek A. 1893. On the occurrence of a Jacobson's organ, with notes on the development of the nasal cavity, the lachrymal duct, and the Harderian gland in Crocodilus porosus. $\mathrm{J}$ Anat Physiol. 27:151-160.

Mills JRE. 1967. A comparison of lateral jaw mowements in some mammals from wear facets on the teeth. Arch Oral Biol. 12:645-661.

Montanucci RR. 1968. Comparative dentition in four iguanid lizards. Herpetologica. 24:305315.

Mueller-Töwe IJ. 2006. Feeding options in Steneosaurus bollensis (Mesoeucrocodylia, Thalattosuchia). Hantkeniana Spec Vol 5:46-48.

Muizon C, Gayet M, Lavenu A, Marshall LG, Sigé B, Villarroel C. 1983. Late Cretaceous vertebrates, including mammals, from Tiupampa, south-central Bolivia. Geobios. 16: $747-753$.

Nash D. 1968. A crocodile from the Upper Triassic of Lesotho. J Zool. 156:163-179

Nash DS. 1975. The morphology and relationships of a crocodilian, Orthosuchus stormbergi, from the Upper Triassic of Lesotho. Ann S Afr Mus. 67:227-329. 
Nesbitt SJ. 2011. The early evolution of archosaurs: relationships and the origin of major clades. Bull Am Mus Nat Hist. 353:1-292 .

Nobre PH. 2004. Morfologia pós-craniana de Candidodon itapecuruense (Crocodylomorpha, Mesoeucrocodylia) do Cretáceo do Brasil. Rev Bras Paleontol. 7(1):87-92.

Nobre PH, Carvalho IS. 2002. Osteologia do crânio de Candidodon itapecuruense (Crocodylomorpha, Mesoeucrocodylia) do Cretáceo do Brasil. In: Simpósio sobre o Cretáceo no Brasil, 6, Simpósio sobre el Cretácico de América de Sur, 2, São Pedro, 2002. Bol Univ Estadual Paulista, Rio Claro. pp. 77-82.

Nobre PH, Carvalho IS. 2006. Adamantinasuchus navae: a new Gondwanan Crocodilomorpha (Mesoeucrocodylia) from the Late Cretaceous of Brazil. Gondwana Res. 10:370-378.

Nobre PH, Carvalho IS, Vasconcellos FM, Nava WR. 2007. Mariliasuchus robustus, um novo Crocodylomorpha (Mesoeucrocodylia) da Bacia Bauru, Brasil. Anu Inst GeociêncUniv Fed Rio de Janeiro. 30:32-42.

Nobre PH, Carvalho IS, de Vasconcellos FM, Souto PR. 2008. Feeding behavior of the Gondwanic Crocodylomorpha Mariliasuchus amarali from the Upper Cretaceous Bauru Basin, Brazil. Gondwana Res. 13:139-145.

Norell MA, Clark JM, Hutchinson JH. 1994. The Late Cretaceous alligatoroid Brachychampsa montana (Crocodylia): new material and putative relationships. Am Mus Novit. 3116:1-26.

Norman DB. 2004. Basal Iguanodontia. In: Weishampel DB, Dodson P, Osmolska H, editors. The Dinosauria (second edition). Berkeley: University of California Press. p. 413-437. Norman DB, Witmer LM, Weishampel DB. 2004. Basal Ornithischia. In: Weishampel DB, Dodson P, Osmolska H, editors. The Dinosauria (second edition). Berkeley, University of California Press. p. 325-334. 
Novas FE, Pais DF, Pol D, Carvalho IS, Scanferla A, Mones A, Riglos MS. 2009. Bizarre notosuchian crocodyliform with associated eggs from the upper Cretaceous of Bolivia. $\mathrm{J}$ Vertebr Paleontol. 29:1-5.

Nowinski A.1971. Nemegtosaurus mongoliensis n. gen., n. sp. (Sauropoda) from the uppermost Cretaceous of Mongolia. Palaeontol Polon. 25:57-81

Nydam RL. 1999. Polyglyphanodontinae (Squamata: Teiidae) from the medial and Late Cretaceous: new taxa from Utah, U.S.A. and Baja California del Norte, Mexico. Utah Geol Surv Misc Publ. 99(1):303-317.

Nydam RL, Gauthier JA, Chiment JJ. 2000. The mammal-like teeth of the Late Cretaceous lizard Peneteius aquilonius Estes 1969 (Squamata, Teiidae). J Vertebr Paleontol. 20(3):628-631.

Nydam RL, Cifelli RL. 2002. A new teiid lizard from the Cedar Mountain Formation (Albian-Cenomanian boundary) of Utah. J Vertebr Paleontol. 22(2):276-285.

Nydam RL, Cifelli RL. 2005. New data on the dentition of the scincomorphan lizard Polyglyphanodon sternbergi. Acta Palaeontol Pol. 50(1): 73-78.

O’Connor PM, Gottfried MD, Stevens NJ, Roberts EM, Ngasala S, Kapilima S, Chami R. 2006. A new vertebrate fauna from the Cretaceous Red Sandstone Group, Rukwa Rift Basin, southwestern Tanzania. J Afr Earth Sci. 44:277-288.

O’Connor PM, Sertich JJW, Stevens NJ, Roberts EM, Gottfried MD, Hieronymus TL, Jinnah ZA, Ridgely R, Ngasala SE, Temba J. 2010. The evolution of mammal-like crocodyliforms in the Cretaceous Period of Gondwana. Nature. 466:748-751.

Ortega F, Gasparini Z, Buscalioni AD, Calvo JO. 2000. A new species of Araripesuchus (Crocodylomorpha, Mesoeucrocodylia) from the lower Cretaceous of Patagonia (Argentina). J Vertebr Paleontol. 20:57-76. 
Osmólska H. 1972. Preliminary note on a crocodilian from the Upper Cretaceous of Mongolia. Palaeontol Pol. 27:43-47.

Owen R. 1874. Monograph on the fossil Reptilia of the Wealden and Purbeck Formations. Suppl. No. 6 (Hylaeochampsa). Paleontogr Soc Monographs. 27:1-7.

Owen R. 1879. Monograph on the fossil Reptilia of the Wealden and Purbeck formations. Supplement no. IX. Crocodilia (Goniopholis, Brachydectes, Nannosuchus, Theriosuchus, and Nuthetes). Palaeontograph Soc. 1879:1-19

Ösi A. 2008. Cranial osteology of Iharkutosuchus makadii, a Late Cretaceous basal eusuchian crocodyliform from Hungary. Neues Jahrb Geol Pal Abh. 248(3):279-299.

Ösi A. 2010. Jaw mechanism, dental occlusion and effective oral food processing in heterodont crocodyliforms: an unexpected variability. $70^{\text {th }}$ Annual Meeting of the Society of Vertebrate Paleontologists, Pittsburgh, USA. J Vertebr Paleontol. 142A.

Ösi A. 2011. Feeding-related characters in basal pterosaurs: implications from jaw mechanism, dental function and diet. Lethaia. 44:136-152.

Ösi A. and Barrett, P. M. 2011. Dental wear and oral food processing in Caiman latirostris: analogue for fossil crocodylians with crushing teeth. Neues Jahrb Geol Pal Abh. 261:201207.

Ösi A, Weishampel DB. 2009. Jaw Mechanism and Dental Function in the Late Cretaceous Basal Eosuchian Iharkutosuchus. J Morphol. 270(8): 903-920.

Ösi A, Clark JM, Weishampel DB. 2007. First report on a new basal eusuchian crocodyliform with multi-cusped teeth from the Upper Cretaceous (Santonian) of Hungary. Neues Jahrb Geol Pal Abh. 243(2):169-177.

Ösi A, Prondvai E, Frey E, Pohl B. 2010. New interpretation of the palate of pterosaurs. Anat Rec. 293:243-258. 
Ösi A, Makádi L, Rabi M, Szentesi Z, Botfalvai G, Gulyás P. 2012. The Late Cretaceous continental vertebrate fauna from Iharkút, western Hungary: a review. In: Godefroit P, editor. Bernissart dinosaurs and Early Cretaceous ecosystems. Indiana: Indiana University Press. p. 533-570

Papakosta M, Bakaloudis D, Kitikidou K, Vlachos C, Chatzinikos E. 2010. Dietary overlap among seasons and habitats of red fox and stone marten in Central Greece. Eur J Sci Res. $45: 122-127$.

Parsons TS. 1970. The nose and Jacobson's organ. In: Gans C, Parsons TS, editors. Biology of the Reptilia, Vol. 2. New York: Academic Press. p. 99-191.

Pascual R, Ortiz-Jaureguizar E. 2007. The Gondwanan and South American episodes: two major and unrelated moments in the history of the South American mammals. J Mammal Evol. 14:75-137.

Pierce SE, Benton MJ. 2006. Pelagosaurus typus Bronn, 1841 (Mesoeucrocodylia: Thalattosuchia) from the Upper Lias (Toarcian, Lower Jurassic) of Somerset, England. J Vert. Paleontol. 26(3):621-635.

Pol D. 2003. New remains of Sphagesaurus huenei (Crocodylomorpha: Mesoeucrocodylia) from the Late Cretaceous of Brazil. J Vertebr Paleontol. 23:817-831.

Pol D. 2005. Postcranial remains of Notosuchus terrestris Woodward (Archosauria: Crocodyliformes) from the Upper Cretaceous of Patagonia, Argentina. Ameghiniana. $42: 21-38$.

Pol D, Apesteguia S. 2005. New Araripesuchus remains from the Early Late Cretaceous (Cenomanian-Turonian) of Patagonia. Am Mus Novit. 3490:1-38.

Pol D, Ji S, Clark JM, Chiappe LM. 2004. Basal crocodyliforms from the Lower Cretaceous Tugulu Group (Xinjiang, China), and the phylogenetic position of Edentosuchus. Cretaceous Res. 25:1-20. 
Pol D, Norell MA. 2004a. A new crocodyliform from Zos Canyon, Mongolia. Am Mus Novit. 3445:1-36.

Pol D, Norell MA. 2004b. A new gobiosuchid crocodyliform taxon from the Cretaceous of Mongolia. Am Mus Novit. 3458:1-31.

Pol D, Powell JE. 2011. A new sebecid mesoeucrocodylian from the Rio Loro Formation (Palaeocene) of north-western Argentina. Zool J Linn Soc. 163:S7-S36.

Pough FH. 1973. Lizard energetics and diet. Ecology. 54:837-844.

Price LI. 1950. On a new Crocodilia, Sphagesaurus from the Cretaceous of State of São Paulo, Brazil. An Acad Bras Ciênc. 22:77-83.

Price LI. 1955. Novos crocodilídeos dos arenitos da Série Baurú, Cretáceo do Estado de Minas Gerais. An Acad Bras Ciênc. 27(4):487-498

Price LI. 1959. Sobre um crocodilídeo notossúquio do Cretáceo Brasileiro. Bol Depart Nac Produc Mineral Geol Mineral. 188:1-55.

Prondvai E, Ösi A. 2011. Potential for intracranial movements in pterosaurs. Anat Rec. 294:813-830.

Rabi M, Ösi A. 2010. Specialized basal eusuchian crocodilians in the Late Cretaceous of Europe: evidence for the hylaeochampsid affinites of Acynodon and its implication on alligatoroid biogeography. Abstract Volume of the 8th Meeting of the European Association of Vertebrate Palaeontologists; Aix-en-Provance, France.

Reisz RR, Sues HD. 2000. Herbivory in Late Paleozoic and Triassic Terrestrial Vertebrates. In: Sues HD, editor. Evolution of Herbivory in Terrestrial Vertebrates. Cambridge University Press. p. 9-41.

Rensberger JM. 1973. An occlusal model for mastication and dental wear in herbivorous mammals. J Paleontol. 47:515-528. 
Robinson PL. 1976. How Sphenodon and Uromastix grow their teeth and use them. In:

Bellairs Ad'A, Cox CB, editors. Morphology and Biology of the Reptiles. Academic Press, London. p. 43-64.

Rogers JV II. (2003). Pachycheilosuchus trinquei, a new procoelous crocodyliform from the Lower Cretaceous (Albian) Glen Rose Formation of Texas. J Vertebr Paleont. 23(1): 128145.

Rossmann T. 2000. Ungewöhnliche Krokodilkonstruktionen der Vorzeit: 2. Krokodile mit Knackzähnen. Stud Int J. 7(2):82-84.

Rougier GW, Martinelli AG, Forasiepi A. 2003. The Mesozoic mammalian record in South America: a reapprasial. Seattle Annual Meeting, Terrestrial Paleobiology of South America, Cretaceous through Neogene, 2003; Seattle.

Rougier GW, Apesteguia S, Gaetano LC. 2011. Highly specialized mammalian skulls from the Late Cretaceous of South America. Nature. 479:98-102.

Rusconi C. 1933. Sobre reptiles cretáceos del Uruguay (Uruguaysuchus aznarezi, n. g. n. sp) y sus relaciones com los notosúquidos de Patagônia. Bol Inst Geol y Perforaciones Montevideo Uruguay. 19:1-64.

Rybczynski N, Reisz RR. 2001. Earliest evidence for efficient oral processing in a terrestrial herbivore. Nature. 411:684-687.

Salisbury SW, Molnar RE, Frey E, Willis PMA. 2006. The origin of modern crocodyliforms: new evidence from the Cretaceous of Australia. P Roy Soc B 273:2439-2448 Sanson GD. 1989. Morphological adaptations of teeth to diets and feeding in the Macropodoidea. In: Grigg G, Jarman P, Hume I, editors. Kangaroos, wallabies and ratkangaroos. NSW Australia: Surrey Beatty and Sons. p 151-168. 
Schumacher GH. 1973. The head muscles and hyolaryngeal skeleton of turtles and crocodilians. In: Gans C, Parson TS, editors. Biology of the Reptilia. London: Academic Press. p. 101-199.

Schwarz D, Fechner R. 2004. Lusitanisuchus, a new generic name for Lisboasaurus mitracostatus (Crocodylomorpha: Mesoeucrocodylia), with a description of new remains from the Upper Jurassic (Kimmeridgian) and Lower Cretaceous (Berriasian) of Portugal. Can J Earth Sci. 41(10):1259-1271.

Schwarz D, Salisbury SW. 2005. A new species of Theriosuchus (Atoposauridae, Crocodylomorpha) from the Late Jurassic (Kimmeridgian) of Guimarota, Portugal. Géobios. 38:779-802

Schwenk K. 2000. Feeding in lepidosaurs. In: Schwenk K, editor. Feeding, Form, Function, and Evolution in Tetrapod Vertebrates. Academic Press, New York. p. 175-291.

Sereno PC, Beck AL, Dutheil DB, Larsson HCE, Lyon GH, Moussa B, Sadleir RW, Sidor CA, Varricchio DJ, Wilson GP, Wilson JA. 1999. Cretaceous sauropods from the Sahara and the uneven rate of skeletal evolution among dinosaurs. Science. 286:1342-1347.

Sereno PC, Wilson JA, Witmer LM, Whitlock JA, Maga A, Ide O, Rowe TA. 2007. Structural Extremes in a Cretaceous Dinosaur. PLoS ONE. 2(11):e1230.

Sereno PC, Larsson HCE. 2009. Cretaceous crocodyliforms from the Sahara. ZooKeys. 28:143.

Smith KK. 1993. The form of the feeding apparatus in terrestrial vertebrates: studies of adaptation and constraint. In: Hanken J, Hall BK, editors. The skull. Chicago: University of Chicago Press. p. 150-196.

Soto M, Pol D, Perea D. 2011. A new specimen of Uruguaysuchus aznarezi Crocodyliformes: Notosuchia) from the middle Cretaceous of Uruguay and its phylogenetic relationships. Zool J Linn Soc Lond. 163:173-198. 
Stecher R. 2008: A new Triassic pterosaur from Switzerland (Central Austroalpine, Grisons), Raeticodactylus filisurensis gen. et sp. nov. Swiss J Geosci. 101:1-17.

Stromer E. 1914. Ergebnisse der Forschungreisen Prof. E. Stromers in den Wusten Agyptens:

II. Wirbeltier-Reste der Baharije-Stufe (unterstes Cenoman). 1. Einleitung und 2.

Libycosuchus. Abh Kon Bay Akad Wiss Math-Phys K1. 27(3):1-15

Sues HD, Clark JM, Jenkins JrF. 1994. A review of the Early Jurassic tetrapods from the Glen Canyon Group of the American southwest. In: Fraser N, Sues H-D, editors. In the shadow of the dinosaurs: Early Mesozoic tetrapods. New York: Cambridge University Press. p. 284-294.

Sullivan RM, Lucas SG. 2003. Brachychampsa montana Gilmore (Crocodylia, Alligatoroidea) from the Kirtland Formation (upper Campanian), San Juan Basin, New Mexico. J Vertebr Paleont 23(4): 832-841.

Sutherland DR, Glen AS, de Tores PJ. 2011. Could controlling mammalian carnivores lead to mesopredator release of carnivorous reptiles? Proc Roy Soc B. 278:641-648.

Szalai E. 2005. Paleomagnetic studies from Iharkút. Unpublished manuscript, Eötvös Loránd University, Department of Physical and Applied Geology. [In Hungarian]

Teaford MA. 1988. A review of dental microwear and diet in modern mammals. Scan Microscopy. 2:1149-1166.

Teaford MF, Oyen OJ. 1989. Live primates and dental replication: New problems and new techniques. Am J Phys Anthropol. 80:73-81.

Throckmorton GS. 1976. Oral food processing in two herbivorous lizards, Iguana iguana (Iguanidae) and Uromastix aegyptus (Agamidae). J Morphol. 148:363-390.

Throckmorton GS. 1979. The effect of wear on the cheek teeth and associated dental tissues of the lizard Uromastix aegyptius (Agamidae). J Morph. 160:195-208. 
Throckmorton GS, Hopson JA. 1981. A redescription of Toxolophosaurus cloudi Olson, a Lower Cretaceous herbivorous sphenodontid reptile. J Paleontol. 55:586-597.

Tomo S, Tomo I, Townsend GC, Hirata K. 2007. Masticatory muscles of the great-grey kangaroo (Macropus giganteus). Anat Rec. 290:382-388.

Tucker AD, Limpus CJ, McCallum HI, McDonald KR. 1996. Ontogenetic dietary partitioning by Crocodylus johnstoni during the dry season. Copeia. 1996: 978-988.

Turner AH. 2006. Osteology and phylogeny of a new species of Araripesuchus

(Crocodyliformes: Mesoeucrocodylia) from the Late Cretaceous of Madagascar. Hist Biol. $18: 255-369$.

Turner AH, Brochu C. 2010. A Reevaluation of the crocodyliform Acynodon from the Late Cretaceous of Europe. J Vertebr Paleontol. 30:178A.

Turner AH, Sertich JJW. 2010. Phylogenetic history of Simosuchus clarki (Crocodyliformes: Notosuchia) from the Late Cretaceous of Madagascar. In: Krause DW, Kley NJ, editors. Simosuchus clarki (Crocodyliformes: Notosuchia) from the Late Cretaceous of Madagascar. J Vertebr Paleont 30(Supplement 1): 177-236.

Ungar PS. 1996. Dental microwear of European Miocene catarrhines: Evidence for diets and tooth use. J Hum Evolution. 31:355-366.

Upchurch P, Barrett PM. 2000. The evolution of sauropod feeding mechanisms. In: Sues HD, editor. Evolution of herbivory in terrestrial vertebrates: perspectives from the fossil record. Cambridge University Press, New York. p 79-122.

Varriale F. 2012. The neoceratopsian horizontal shelf is not horizontal, and other new information about this structure. $72^{\text {th }}$ Annual Meeting of the Society of Vertebrate Paleontologists, Raleigh, USA. J Vertebr Paleontol. 188A. 
Vasconellos FM, Carvalho IS. 2005. Estágios de desenvolvimento de Mariliasuchus amarali, Crocodyliformes Mesoeucrocodylia da Formação Adamantina, Cretáceo Superior da Bacia Bauru, Brasil. Anu Inst Geociên. 28:49-69.

Vickaryous MK, Maryanska T, Weishampel DB. 2004. Ankylosauria. In: Weishampel DB, Dodson P, Osmolska H, editors. The Dinosauria (second edition). Berkeley, University of California Press. p. 363-392

Walker AD. 1961. Triassic reptiles from the Elgin area: Stagonolepis, Dasygnathus and their allies. Philosophical Transactions of the Royal Society of London, Series B 244:103-204. Walker AD. 1990. A revision of Sphenosuchus acutus Haughton, a crocodylomorph reptile from the Elliot Formation (Late Triassic or Early Jurassic) of South Africa. Philos T R Soc Lond B. 330:1-120 .

Walker CA, Hoeck HN, Perez L. 1978. Microwear of mammalian teeth as an indicator of diet. Science. 201:808-810.

Weijs WA. 1975. Mandibular movements of the albino rat during feeding. J Morph. 145: $107-124$.

Weijs WA. 1994. Evolutionary approach of masticatory motor patterns in mammals. In: Bels V, Chardon M, Vandewalle P, editors. Biomechanics of Feeding in Vertebrates. SpringerVerlag, Berlin. p. 281-320.

Weishampel DB. 1984. Evolution of Jaw Mechanisms in Ornithopod Dinosaurs. Adv Anat Embr Cell Biol. 87:1-109.

Weitzel K. 1935. Hassiacosuchus haupti n.g n.sp. ein durophages Krokodil aus dem Mitteleozän von Messel. Not Ver Erdk hess geol Landesanst Darmstadt. 16:40-49. Whetstone KN, Whybrow PJ. 1983. A “cursorial” crocodilian from the Triassic of Lesotho (Basutoland), southern Africa. Occ P Mus Nat Hist. The University of Kansas 106:1-37. 
Wild R. 1978: Die Flugsaurier (Reptilia, Prerosauria) aus der oberen Trias von Cene bei Bergamo, Italien. Bollettino della Società Paleontologica Italiana 17:176-256.

Willis PMA, Mackness BS. 1996. Quinkana babarra, a new species of ziphodont mekosuchine crocodile from the Early Pliocene Bluff Downs Local Fauna, Northern Australia with a revision of the genus. Proc Linn Soc N S W. 116:143-151.

Witmer LM. 1997. The evolution of the antorbital cavity of archosaurs: a study in soft-tissue reconstruction in the fossil record with an analysis of the function of pneumaticity. Mem Soc Vert Paleont- J Vertebr Paleont. 17(Supplement to 1):1-73.

Wood AE. 1965. Grades and clades among rodents. Evolution 19:115-130.

Woodward AS. 1896. On two Mesozoic crocodilians from the red sandstones of the territory of Neuquen (Argentina Republic). Notosuchus (genus novum) and Cynodontosuchus (genus novum). Anal Mus La Plata. Paleontol. 4:1-20.

Wu XC, Brinkman DB, Russell AP. 1996. A new alligator from the Upper Cretaceous of Canada and the relationships of early eusuchians. Palaeontology. 39:351-375.

Wu XC, Brinkman DB, Lu JC. 1994. A new species of Shantungosuchus from the Lower Cretaceous of Inner Mongolia (China), with comments on S. chuhsienensis Young, 1961 and the phylogenetic position of the genus. J Vertebr Paleontol. 14:210-229.

Wu XC, Sues HD. 1996. Anatomy and phylogenetic relationships of Chimaerasuchus paradoxus, an unusal crocodyliform reptile from the Lower Cretaceous of Hubei, China. J Vertebr Palaeontol. 16(4):688-702.

Wu XC, Sues HD, Sun A. 1995. A plant-eating crocodyliform from the Cretaceous of China. Nature. 376:678-680.

Young CC. 1973. A new fossil crocodile from Wuerho. Memoirs of the Institute of Vertebrate Paleontology and Paleoanthropology 11:37-44 (in Chinese). 
El-Zaatari S, Grine FE, Teaford MF, Smith HF. 2005. Molar microwear and dietary reconstructions of fossil Cercopithecoidea from the PlioPleistocene deposits of South Africa. J Hum Evol. 49:180-205.

Zaher H, Pol D, Carvalho AB, Riccomini C, Campos D, Nava W. 2006. Redescription of the cranial morphology of Mariliasuchus amarali, and its phylogenetic affinities (Crocodyliformes, Notosuchia). Am Mus Novit. 3512:1-40.

\section{Figure captions:}

Figure 1. The relative size of moment arms (c) in mammals (after Smith, 1993) and crocodylians (Ösi and Weishampel 2009). A, mandible of an ungulate. B, Mandible of a carnivore mammal. C, Mandible of Iharkutosuchus makadii. D, Mandible of Pelagosaurus typus (redrawn from Pierce and Benton 2006). Mandibles of mammals and crocodylians are scaled to the same size. Note that when the jaw joint is situated highly dorsally above the occlusal plane, the relative size of moment arms of the muscles originating deep ventrally (m. masseter of mammals, MPT, MAMP of crocodylians) increases. Distance between the occlusal plane and the jaw joint is indicated with ' $a$ ', the relative size of the moment arm of 
m. temporalis with 'b', the relative size of the moment arm of m. masseter with 'c', the relative size of the moment arm of $\mathrm{m}$. adductor mandibulae externus profundus (MAMEP) and m. pseudotemporalis (MPS) with 'd', and the relative size of the moment arm of the pterygoid muscles (MPT) and m. adductor mandibulae posterior (MAMP) with 'e'. Figure 2. Skull of different heterodont crocodyliforms demonstrating the variability of proportions, shape and size of the rostrum and cranial openings. Not to scale.

Figure 3. Divesity in the dentition of heterodont crocodyliforms. A, Mariliasuchus amarali posterior tooth. B, Edentosuchus tienshanensis left mandibular tooth row. C, Armadillosuchus arrudai 4th right dentary tooth. D, Candidodon itapecuruense posterior maxillary tooth. E, Sphagesaurus montealtensis maxillary tooth row. F, Chimaerasuchus paradoxus left multicusped maxillary tooth. G, Malawisuchus mwakasyungutiensis maxillary tooth. H, Iharkutosuchus makadii posterior left maxillary teeth. I, Unasuchus reginae isolated posterior crushing tooth. J, Bernissartia fagesii left posterior crushing teeth. K, Simosuchus clarki anterior mandibular teeth. L, Dentition of the left mandible of Allognathosuchus haupti. Figure 4. Origin and insertion areas of the most important cranial adductor muscles in Alligator mississippiensis (after Iordansky 1964, Busbey 1989, Holliday and Witmer 2007). A, Origin surfaces of the most important cranial adductor muscles in the temporal region in ventral view. B, Origin surfaces of MAMEP in the supratemporal region in dorsal view. C, Insertion areas of the most important cranial adductor muscles on the post dentary part of the mandible in medial view. $\mathrm{D}$, Representative lines of action of the major jaw adductors in dorsal and $\mathrm{E}$, and lateral view.

Figure 5. Proal and palinal movement in extant vertebrates. A-F, proal movement (protractive powerstroke) in Sphenodon with particular phases of one chewing cycle (A-D), the morphology of the mandibular glenoid surface with the position of the quadrate condyles at the beginning of the chewing cycle (E), and the orientation of pterygoid muscles (F) pulling 
the mandibles forward during jaw closure and occlusion (Gorniak et al. 1982, Jones et al. 2012). G-I, proal movement in the rat with the origin and insertion surfaces of masseter muscles $(\mathrm{G})$ responsible for the protraction of the mandibles. H-I, the movements of the molars across each other during the powerstroke of one chewing cycle. The solid lines indicate the respective positions of the upper and lower molars at the start of the powerstroke, the dashed line the position of the lower molars at the end of the powerstroke in lateral $(\mathrm{H})$ and occlusal view. The crosses represent the $\mathrm{M}_{1}$ marker at both positions, while the dots indicate the marker in the intermediate frames (modified after Weijs 1975). J-O, Palinal movement (retractive powerstroke) in Gopherus with particular phases of one chewing cycle (J-M), the morphology of the mandibular glenoid surface with the position of the quadrate condyles at the beginning of the chewing cycle $(\mathrm{N})$, and the orientation of external adductor muscles $(\mathrm{O})$ pulling the mandibles backward during jaw closure and occlusion (modified after Bramble 1974). The inserts (in A-D and J-M) show the path of the tip of the mandible (with green point) relative to the head.

Figure 6. Four basic types of mandibular movement occured during the evolution of the Crocodyliformes. A-E, Phases of orthal jaw movement. The mandible opens (A) and closes (B), and the quadrate condyles precisely fit into the complementary surface of the glenoid surface (C). D, The position of the upper and lower teeth in lateral (left) and mesiodistal (right) views when occlusion is not present. E, The position of the upper and lower teeth in medial (left) and mesiodistal (right) views when occlusion is present. F-J, Phases of proal movement. The mandible opens and slightly moves backward (F) then closes (G), and at the end of the closing phase, in a separated step, it moves anteriorly $(\mathrm{H})$. I, The glenoid surface is anteroposteriorly more elongated than the quadrate condyles. J, Shearing contact of the teeth (the lower tooth moves anteriorly) in proal movement. $\mathrm{K}-\mathrm{O}$, Phases of palinal movement. The mandible opens and slightly moves anteriorly $(\mathrm{K})$ then closes $(\mathrm{L})$, and at the end of the 
closing phase it moves backward and slightly upward (M). N, The glenoid surface is anteroposteriorly more elongated than the quadrate condyles; O, Shearing contact of the teeth (the lower tooth moves backward and upward) in palinal movement. P-T, Phases of lateromedial mandibular movement. The mandible opens $(\mathrm{P})$ then closes $(\mathrm{Q})$, and at the end of the closing phase it rotates lateromedially (R). S, The mechanism of lateromedial mandibular movement in the jaw joint (i.e. rotation of the mandible around the quadrate condyle). T, Tooth-tooth occlusion of posterior grinding teeth in lateromedial mandibular movement.

Figure 7. The protosuchian Kayenta form (UCMP 97638) from the Early Jurassic Glen Canyon Group, Arizona, USA. Skull in dorsal (A), occipital (B), lateral (C), and ventral (D) views. Mandible in occlusal (E) and left lateral (F) views.

Figure 8. Dentition of the protosuchian Kayenta form (UCMP 97638) from the Early Jurassic Glen Canyon Group, Arizona, USA. Rigth maxillary tooth row in ventrolateral (A) and lateral-ventrolateral (B) views. C, Right mandibular dentition in occlusal view. D, SEM close-up of right 3rd bicusped maxillary tooth crown. E, Posterolateral view of right 3rd and 4th bicusped maxillary teeth. F, Oblique view of the right 4th and 5th bicusped teeth. G, Details of the worn occlusal surface of the right 4th maxillary tooth. The "bubbles" on E, F, G are artifacts.

Figure 9. Reconstruction of the origin and insertion areas of the most important cranial adductor muscles in the protosuchian Kayenta form from the Early Jurassic Glen Canyon Group, Arizona, USA. A, Origin surfaces of MPTV, MPTD and MAMP in ventral view. B, Origin and insertion areas of the most important cranial adductor muscles in lateral view. Figure 10. Tooth rows and occlusion map of the teeth of the protosuchian Kayenta form from the Early Jurassic Glen Canyon Group, Arizona, USA. A, Mandibular tooth row (wear facets with grey). B, Maxillary tooth row (wear facets with grey). C, Position of mandibular teeth 
(red outlines) relative ot the maxillary teeth (in grey). D, Position of mandibular teeth (red outlines) relative ot the maxillary teeth (wear facets in grey). E, Position and orientation of the maxillary (grey) and dentary teeth relative to each other in mesiodistal view. F, Position and orientation of the maxillary (grey) and dentary teeth relative to each other in lateral view. Figure 11. Edentosuchuss tienshanensis (IVPP V3236 (1, 2), holotype) from the Early Cretaceous of China. A, Skull table in dorsal view. Fused left and right mandibles in occlusal (B), ventral (C), left lateral (D), and right lateral (E) views.

Figure 12. Edentosuchuss tienshanensis (GMPKU-P 200101) from the Early Cretaceous of China. Skull in dorsal (A), lateral (B), and ventral (C) views. D, Anterior part of the left tooth row. E, Posterior left molariform maxillary teeth. F, Posterior right molariform maxillary teeth. G, Anterior part of the right tooth row.

Figure 13. Malawisuchus mwakasyungutiensis from the Early Cretaceous of Africa. Skull (MAL-49) in dorsal (A), ventral (B), and left lateral (C) views. D, Left tooth row in anterolateral view. E, Skull (MAL-46) in left lateral view. Upper molariform tooth in lingual (F) and occlusal (G) views.

Figure 14. Reconstruction of the origin and insertion areas of the most important jaw adductor muscles in Malawisuchus mwakasyungutiensis from the Early Cretaceous of Africa. A, Origin surfaces of MAMEP (A) and MPTV (B). C, Origin and insertion areas of some jaw adductor muscles in lateral view.

Figure 15. Chewing cycle of Malawisuchus mwakasyungutiensis. A, The cylce starts with the opening and slight backward shifting of the mandibles. B, In the beginning of the closing phase, the mandible still shifts backwards. C, The mandible is in a closed position so that the upper and lower teeth come into contact. D, The mandible shifts forward bringing the upper and lower molariform teeth into a shearing contact. E, Shearing contact of the upper and 
lower molariform teeth when the mandible shifts forward. Red point indicates the pivot point of the lower jaw movement.

Figure 16. Chimaerasuchus paradoxus (IVPP V8274) from the Early Cretaceous of China. Rostrum in dorsal (A), ventral (B), lateral (C), and anterior (D) views.

Figure 17. Dentition of Chimaerasuchus paradoxus (IVPP V8274) from the Early Cretaceous of China. Two isolated caninifom (A, B) and one isolated molariform (C) tooth. D, 2nd left maxillary tooth in mesial view. E, 2nd and 3rd maxillary teeth in ventromedial view. F, 2nd and 3rd maxillary teeth in occlusal view. G, 2nd and 3rd maxillary teeth in ventrolateral view. $\mathrm{H}, 2$ nd maxillary tooth in ventromedial view. I, Details of the largest, mesial cusp of the central row of cusps on the 2 nd left maxillary tooth. J, Details of the wear facet on the largest, mesial cusp of the central row of cusps on the 2 nd left maxillary tooth.

Figure 18. Notosuchus terrestris from the Late Cretaceous of Argentina. Skull (RN 1037) in dorsal (A), ventral (B), and left lateral (C) views. D, Secondary bony palate (RN 1040) in dorsal view. E, Right upper dentition in lateral $(\mathrm{E})$ and ventrolateral $(\mathrm{F})$ views.

Figure 19. Dentition and quadrate-mandibular joint in Notosuchus terrestris. A, Upper dentition with wear facets (grey). B, Lower dentition with wear facets (grey). C, The position of the lower dentition (red outlines) relative to the upper dentition. D, Occlusion of upper and lower teeth with ortal movement. E, Occlusion of upper and lower teeth with proal movement. F, Left quadrate-mandible joint in medial view. G, Left quadrate-mandible joint in dorsal view. The red line indicates the border of the glenoid surface.

Figure 20. Mariliasuchus amarali (MZSP-PV-51) from the Upper Cretaceous of Brazilia. Skull in dorsal (A), anterior (B), occipotal (C), ventral (D), and lateral (E) views. Fused mandible in left lateral $(\mathrm{F})$, ventral $(\mathrm{G})$, and dorsal $(\mathrm{H})$ views. Figure 21. Dentition of Mariliasuchus amarali. A, Right upper dentition (MZSP-PV-51). B, Left lower dentition (MZSP-PV-51). C, Right upper posterior molariform teeth (MZSP-PV- 
51). D, Right lower posterior molariform teeth (MZSP-PV-51). E, Left upper posterior molariform teeth (MZSP-PV-51). F, Left lower posterior molariform teeth (MZSP-PV-51). G, Left upper dentition of UFRJ 106-R in medial view. H, Right upper dentition of UFRJ 106-R in medial view. I, Upper dentition of MN 6756 in posterior view. J, Lower dentition of MN 6756 in posterior view.

Figure 22. Overview on the dentition of Mariliasuchus amarali. A, Dentition of MZSP-PV-51 in right lateral view when mandibles are not completely closed (A) and in a fully closed position (B). C, Labial wear facet on the upper left caniniform in MZSP-PV 50. D, Labial and apical wear on the lower anterior teeth of MZSP-PV 50 in ventral view. E, Labial and apical wear on the lower anterior teeth of MZSP-PV 50 in anterior view.

Figure 23. SEM images of the wear pattern on the teeth of Mariliasuchus amarali. A, Lingual wear on the upper caniniform tooth (UFRJ 106 R). B, Lingual wear on the left 3rd maxillary tooth (UFRJ $106 \mathrm{R}$ ). C, Wear pattern of the right maxillary posterior tooth (MZSP-PV-51) in occlusal view. D, Details of the wear facet of the right maxillary posterior tooth (MZSP-PV51). E, Wear pattern of the right maxillary posterior tooth (MZSP-PV-51) in lingual view ("bubbles" are artifacts). F, Wear pattern of the 5th right maxillary tooth (MZSP-PV-51) in lingual view. G, Details of the wear facet of the 5th right maxillary tooth (MZSP-PV-51) in lingual view. $\mathrm{H}$, Wear pattern of the left 6 th mandibular tooth in occlusal view showing the last stage of wear in Mariliasuchus. I, Details of the wear pattern of the 5th right maxillary tooth (MZSP-PV-51).

Figure 24. Reconstruction of the origin and insertion areas of the most important jaw adductor muscles in Mariliasuchus amarali from the Late Cretaceous of Brazil. A, Origin surfaces of jaw adductors in the temporal region in ventral view. B, Origin surfaces of MAMEP in dorsal view. C, Insertion areas of some jaw adductor muscles in medial view. D, Line of action of 
different jaw adductors when the mandibles are open (for color coding see Fig. 4). The glenoid surface is indicated with a red line.

Figure 25. Chewing cycle of Mariliasuchus amarali. A, The cylce starts with the opening of the mandibles. B, In the beginning of the closing phase, the mandible shifts forward. $\mathrm{C}$, When the mandible is in a closed position, the upper and lower teeth come into contact. D, The mandible is pulled upward and backward bringing the lingual surface of the upper and labial surface of the lower molariform teeth into a shearing contact. E, The interaction of the upper and lower molariform teeth when the mandible shifts upward and backward. The solid lines indicate the respective position of the upper and lower molariform teeth. Dashed lines show the position of the lower molariform teeth at the end of the powerstroke. Wear facets on the lower teeth are in grey. The crosses connected with solid lines represent the shifting route of one of the lower molariform teeth during the palinal powerstroke. F, Right quadratemandibular joint of Mariliasuchus amarali in medial view in open (F) and closed (G) position. The red line marks the glenoid surface and the red point indicates the pivot point (jaw joint).

Figure 26. Armadillosuchus arrudai from the Late Cretaceous of Brazil. Skull (UFRJ 303R) in dorsal (A), ventral (B), and left lateral (C) views. D, Rostrum and corresponding part of the fused mandibles (MPMA-64-0001-04) in occluding position in ventral view. E, Anterior fragment of the rostrum (MPMA-64-0001-04) in lateral view. F-G, Anterior fragment of the fused mandibles (MPMA-64-0001-04) in left (F) and right (G) lateral views. H, Rostrum and corresponding part of the fused mandibles (MPMA-64-0001-04) in occluding position on the left side in posterior view. Note that when the teeth of the left side are in occlusion then those of the right side are not close enough to come into occlusion indicating the presence of an alternating dental occlusion in Armadillosuchus (Marino and Carvalho 2009). 
Figure 27. Upper dentition and their wear facets in Armadillosuchus arrudai (MPMA-640001-04) from the Late Cretaceous of Brazil. A-B, Worn right maxillary teeth in occlusal (A) and lingual (B) views. C-E, Details of the lingual wear facets on the right maxillary teeth. FG, Right upper caniniform tooth with lingual wear facets in lingual (F) and ventral (G) views. $\mathrm{H}$, Details of the lingual wear facets of the apical region of the right upper caniniform tooth. Figure 28. Lower dentition and wear in Armadillosuchus arrudai (MPMA-64-0001-04) from the Late Cretaceous of Brazil. A, 4th right dentary tooth in labial view. B, 4th left dentary tooth in dorsolabial view. C, Anterior part of the fused mandibles of MPMA-64-0001-04 in anterolateral view. D, Details of the apical and labial wear facet of the 4th left dentary tooth in labial view.

Figure 29. Sphagesaurus montealtensis (MPMA 15-001/90) from the Late Cretaceous of Brazil. Skull in dorsal (A), ventral (B), and right lateral (C) views. Fused mandibles in dorsal (D), ventral (E), and right lateral (F) views. G, Close-up of the right posterior maxillary teeth in lingual view.

Figure 30. Sphagesaurus sp. ('Caipirasuchus paulistanus' Iori and Carvalho, 2011) (MPMA 67-0001/00) from the Late Cretaceous of Brazil. Skull in dorsal (A), anterior (B), ventral (C), occipital (D), and left lateral (E) views. F, The left temporal region in lateroventral view. Fused mandibles in dorsal $(\mathrm{G})$ and right lateral $(\mathrm{H})$ views.

Figure 31. Dentition and dental wear of Sphagesaurus ('Caipirasuchus paulistanus' Iori and Carvalho, 2011) (MPMA 67-0001/00) from the Late Cretaceous of Brazil. A, Upper dentition in occlusal view. B, Right posterior maxillary teeth in medial view. C-D, Details of the worn surface of some posterior maxillary teeth. Note that the relatively smooth wear facets are devoid of scratches.

Figure 32. Reconstruction of the origin and insertion areas of the most important jaw adductor muscles in Sphagesaurus from the Late Cretaceous of Brazil. A, Origin surfaces of jaw 
adductors in the temporal region in ventral view. B, Origin surfaces of MAMEP in dorsal view. C, Insertion areas of some jaw adductor muscles in medial view.

Figure 33. Chewing cycle of Sphagesaurus. A, The cylce starts with the opening of the mandibles with a slight posterior component in the lower jaw movement. B, In the beginning of the closing phase the mandible shifts forward. $\mathrm{C}$, The mandible is pulled upward and slightly backward into a closed position whereby the upper and lower teeth on one side come into contact. $\mathrm{D}$, The mandible is pulled dominantly backward bringing the lingual surface of the upper and labial carinate surface of the lower molariform teeth into a shearing contact. Red point indicates the pivot point of the mandibular movement. E-H, Position of the mandibular tooth rows (in red) relative to the upper ones during the powerstroke. The black and red dots show the centerpoint of the glenoid surface. E, In this phase the mandible occupies its posteriormost position. F, The mandible is slightly open, moves forward and occupies its anteriormost position. G, The fused mandibles rotate lateromedially around one of the quadrate condyles and bring one side of the upper and lower teeth into contact. $\mathrm{H}$, The mandibles move backward to produce shearing contact between the carinate surfaces of the occluding teeth.

Figure 34. Adamantinasuchus navae (UFRJ-DG 107-R) from the Late Cretaceous of Brazil. Skull and mandible in right lateral (A), dorsal (B), and left lateral (medial) (C) views. D, Details of the rostrum in right lateral view. Right upper molariform teeth in linguoventral (E) and ventral $(\mathrm{F})$ views.

Figure 35. Yacarerani boliviensis (MNK-PAL5063) from the Late Cretaceous of Bolivia. Skull and left mandible in dorsal (A), ventral (B), right lateral (C), and occipital (D) views. E, The anterior part of the dentition of the rostrum in right lateral view and of the left dentary in medial view. F, Skull and left mandible in anterior view. 
Figure 36. Chewing cycle of Yacarerani. A, The cylce starts with the opening of the mandibles. B, In the beginning of the closing phase, the mandible shifts forward. $\mathrm{C}$, The mandible is pulled upward and slightly backward into a closed position whereby the upper and lower teeth come into contact. D, The mandible is pulled dominantly backward and slightly upward bringing the distolingual surface of the upper and mesiolabial cuspidate surface of the lower molariform teeth into contact. Red point indicates the pivot point. E, Lower dentition. F, Upper dentition. G, Position of the mandibular tooth rows (in red) relative to the upper ones during the beginning of the powerstroke. $\mathrm{H}$, The mandibles move backward to produce grinding contact between the cuspidate surfaces of the occluding upper and lower teeth.

Figure 37. Candidodon itepecuruense (UFRJ DG 114-R) from the Early Cretaceous of Brazil. Skull in dorsal (A), ventral (B), left lateral (C), anterior (D), and posterior (E) views. Figure 38. Dentition of Candidodon itepecuruense (UFRJ DG 114-R) from the Early Cretaceous of Brazil. A, Right maxillary dentition in lingual view. B, Left maxillary dentition in lingual view. C, Details of the right posterior teeth in lingual view. D, Right 5 th and 6th maxillary teeth in distolingual view. E, Left 4th maxillary tooth in lingual view ("bubbles" are artifacts). F, Left 6th maxillary tooth in lingual view. G, Details of the apicolingual wear facet of the left 4th maxillary tooth in lingual view. Note the step relationship of EDI. H, Details of the mesial wear facet of the left 6th maxillary tooth in mesiolingual view.

Figure 39. Pakasuchus kapilimai (RRBP 08631) from the Early Cretaceous of Africa. Skull and mandible in dorsal (A) and left lateral (B) views.

Figure 40. Libycosuchus brevirostris (BSM 1917 VIII 574a) from the Late Cretaceous of North Africa. Skull in dorsal (A), ventral (B), right lateral (C), and occipital (D) views. Fused mandibles in right lateral (E) and dorsal (F) views. 
Figure 41. Simosuchus clarki (UA 8679) from the Late Cretaceous of Madagascar. Skull in dorsal (A), ventral (B), and right lateral (C) views. Mandible in dorsal (D) and right lateral (E) views. F, Premaxillary teeth in labial view. G, Posterior tooth crown in labial view.

Figure 42. Reconstruction of the origin and insertion areas of the most important jaw adductor muscles in Simosuchus from the Late Cretaceous of Madagascar. A, Origin surfaces of some jaw adductors in the temporal region in ventral view. B, Origin surfaces of MAMEP in dorsal view. C, Insertion areas of some jaw adductor muscles in medial view.

Figure 43. Bernissartia fagesii (IRScNBr 46) from the Early Cretaceous of Belgium. Skull in dorsal (A) and ventral (B) views, and skull and mandible in left lateral (C) view. D, Details of the posterior section of the left upper and lower tooth rows in lateral view.

Figure 44. Caiman latirostris (NHM RR 2009.2). Skull in dorsal (A), ventral (B), and lateral (C) views. D, The left temporal region in lateroventral view. Mandible in dorsal (E) and left lateral $(\mathrm{F})$ views.

Figure 45. Allognathosuchus haupti from the Eocene of Germany. Skull (HLMD ME 5261) (A) and reconstructed explanatory drawing of the skull (B) in dorsal view. C, Skull and mandible (HLMD ME 4445) in left lateral view. Reconstruction of the skull and mandible in left lateral view. E, Details of the posterior section of the left upper and lower tooth rows in lateral view. Right fragmentary mandible (HLMD ME 5262) in medial (F) and dorsal (G) views. H, Left dentary (HLMD ME 4415) in lateral view. I, Isolated posterior crushing teeth in lingual (I) and dorsolingual ( $\mathrm{J})$ views.

Figure 46. Dentition of Bernissartia from the Early Cretaceous of western Europe. A, Left posterior maxillary teeth of Bernissartia fagesii (IRScNBr 46) in occlusal view. B, Isolated teeth of Bernissartia sp. (BMNH R 9296) in occlusal view from the Barremian of England. C, Globular tooth of Bernissartia sp. (BMNH R 9296 (1)) in occlusal view. D, Posterior crushing tooth of Bernissartia sp. (BMNH R 9296 (2)) in mesiolabial view. E, Close-up of the 
eroded tooth crown of Bernissartia sp. (BMNH R 9296 (2)) in oblique view. F, Posterior crushing tooth of Bernissartia sp. (BMNH R 9296 (3)) in oblique view. G, Close-up of the eroded tooth crown of Bernissartia sp. (BMNH R 9296 (3)) in oblique view ("bubbles" are artifacts).

Figure 47. Teeth and dental wear in Unasuchus reginae and Brachychampsa sp. Isolated tooth (IPFUB 102/11.13) of Unasuchus reginae from the Early Cretaceous of Portugal (A) and close-up of its wear facet (B) ("bubbles" are artifacts). Isolated tooth (IPFUB 102/11.14) of Unasuchus reginae (C) and close-up of its wear facet (D). Isolated tooth (BMNH R8402) assigned to Brachychampsa from the Late Cretaceous of North America (E) and close-up of its wear facet $(\mathrm{F})$.

Figure 48. Dentition and wear patterns in Caiman latirostris (modified from Ösi and Barrett 2011). A, Eroded right posterior crushing teeth in the upper and lower jaws in specimen NHM RR 2009.1. B, Completely eroded tooth crowns straddled by more complete crowns missing only the enamel (15th and 17th) in specimen NHM RR 2009.2. C, Irregular wear facet with the residual dentine bump (11th tooth) in the right maxilla of NHM RR 2009.1 ("bubbles" are artifacts). D, Eroded 11th tooth crown in the right dentary of NHM RR 2009.1. E, Extended wear facet with heavy pits and scratches on the dentine of the 11th maxillary tooth of NHM RR 2009.1. F, Wear facet with irregular enamel-dentine interface of the 11th maxillary tooth of NHM RR 2009.1 (“bubbles” are artifacts).

Figure 49. Jaw mechanism in Allognathosuchus haupti. A, The mandibles are open and ready to capture the food. B, Mandibles are closed by simple orthal jaw closure. There is no anteroposterior or lateromedial sliding of the jaws. Red point indicates the pivot point (jaw joint). Occlusion of the posterior crushing teeth in lateral (C) and mesial (D, E) views. 
Figure 50. Iharkutosuchus makadii from the Late Cretaceous of Hungary. Skull (MTM 2006.52.1.) in dorsal (A), ventral (B) and left lateral (C) views. Left complete mandible (MTM PAL2012.29.1.) in lateral (D), occlusal (E) and medial (F) views.

Figure 51. Dentition of Iharkutosuchus makadii from the Late Cretaceous of Hungary. Isolated anterior incisiviform tooth (MTM 2006.80.1.) in lingual (A) and mesial (B) views. Right 15 th to 11 th multicusped maxillary teeth (MTM 2006.57.1.) in lingual (C), occlusal (D), and labial (E) views. F, Posterior (17th, 18th) grinding teeth (MTM 2006.53.1) in occlusal view. G, Completely worn posterior grinding teeth of the holotype skull (MTM 2006.52.1) in occlusal view. Numbers indicate tooth positions.

Figure 52. Different stages of wear on the supposed anterior incisiviform teeth of Iharkutosuchus makadii (MTM 2006.80.1., from Ösi and Weishampel 2009) in lateral (A-D) and occlusal $(\mathrm{E}-\mathrm{H})$ views.

Figure 53. Macrowear patterns on the teeth of Iharkutosuchus makadii (modified from Ösi and Weishampel 2009). A, Strongly worn posterior molariform tooth with short and poorly oriented scratches. B, Mesiodistally oriented scratches on a left isolated 17th maxillary tooth. C, Poorly oriented scratches on the mesiolingual part of a left isolated 17th maxillary tooth. D, Labiolingually oriented scratches on the dentine of the left 17th tooth in holotype (MTM 2006.52.1) maxilla. E, Labiolingually oriented scratches on the dentine of the left 15 th tooth in holotype maxilla. Note the deep area between the 15th and 16th teeth worn by the occluding opposite lower tooth. F, Almost labiolingually oriented, long, parallel scratches on the dentine of the right 16 th tooth in holotype maxilla. G, Labiolingually and mesiolabialdistolingually oriented, parallel scratches on the dentine of the right 17 th tooth in maxilla of the juvenile skull (MTM 2006.56.1). H, Close-up of scratches on the dentine of the right 17th tooth in maxilla of the juvenile skull (MTM 2006.56.1). 
Figure 54. Scanning electron micrographs of the occlusal surface of the 17 th teeth Iharkutosuchus (MTM 2006.80.1., modified from Ösi and Weishampel 2009). A, Position of the microwear sites $(\mathrm{B}, \mathrm{C}, \mathrm{D})$ on the studied left 17 th tooth. Directions are indicated for the tooth and the micrographs. B, Micrograph from the lingual side of the crown, scratches show a nearly mesiodistal orientation. $\mathrm{C}$, Micrograph from the labial side of the main cusp shows a high number of scratches with variable orientations. D, Micrograph from the lingual side of the crown. E, Position of the microwear site $(\mathrm{F})$ on the studied right 17 th tooth. F, Micrograph from a worn secondary cusp, note the strong labiolingual scratches.

Figure 55. Reconstruction of the origin and insertion areas of the most important jaw adductor muscles in Iharkutosuchus from the Late Cretaceous of Hungary. A, Origin surfaces of some jaw adductors in the temporal region in ventral view. B, Insertion areas of some jaw adductor muscles on the mandible in medial view.

Figure 56. Diagramatic sketch of the dental occlusion and oral food processing by the teeth of Iharkutosuchus makadii (modified from Ösi and Weishampel 2009). Initial contact between upper (in grey) and lower premolariform and anterior molariform teeth in lateral (A) and occlusal (B) views. C, Initial contact between upper (16th and 17th) and lower (13th to 15th) molariform teeth in occlusal view. D, One cycle of occlusion between anterior teeth (arrows show the direction of movement of the lower tooth). E, One cycle of occlusion between the largest, 17th (upper) and 5th (lower) teeth. F-G, The reconstructed upper and lower dentitions of Iharkutosuchus makadii superimposed in centric relation (F) and in the middle of the chewing stroke $(\mathrm{G})$. In this phase, lateral jaw movement is accomplished by rotation of the mandibles about the right quadrate condyle. No symphyseal movement is shown here. Heavy lines in the tooth row $(\mathrm{G})$ showing the path and distances of the mandibular teeth relative to the upper teeth. 
Figure 57. Acynodon iberoccitanus from the Late Cretaceous of France. Skull (ACAP-FX1) in dorsal (A), ventral (B), left lateral (ACAP-FX2) (C), and occipital (ACAP-FX1) (D) views. Left mandible (ACAP uncatalogued) in lateral (E), medial (F), and occlusal (G) views. Figure 58. Dentition of Acynodon. A, Acynodon iberoccitanus spatulated anterior maxillary teeth (ACAP-FX2). B, Close-up view of spatulated anterior maxillary teeth in Acynodon iberoccitanus. C-D, Posterior left maxillary crushing teeth of Acynodon iberoccitanus in occlusal (C) and mesiolingual (D) views. E, Posterior right maxillary crushing teeth of Acynodon iberoccitanus in labial view. F, Posterior left maxillary crushing teeth of Acynodon adriaticus (MCSNT 57248) in labial view. G, Premaxillary conical teeth of Acynodon adriaticus in labial view.

Figure 59. The occurrence of heterodonty and different types of jaw mechanisms mapped onto the phylogenetic relationships of the Crocodyliformes. The phylogenetic tree is based on the composition of the results of Pol and Powell (2011), Andrade et al. (2011) and Brochu (2011).

Figure 60. The occurrence of heterodont dentition and different types of jaw mechanisms mapped onto four different interpretations of the phylogentic relationships of Notosuchia. Note that in all interpretations, both proal and palinal jaw movements appeared at least two times independently during the evolution of Notosuchia.

Figure 61. Different food preferences suggested on the basis of craniodental features mapped onto the phylogenetic hypothesis of Iori and Carvalho (2011). Body length is calculated from the avaliable postcranial material or adopted from literature. 
Table 1. Measurements and data on heterodont crocodyliforms used in this study. 


\begin{tabular}{|c|c|c|c|c|c|c|c|c|}
\hline Taxon & $\begin{array}{c}\text { skull } \\
\text { length } \\
\text { (cm) }\end{array}$ & $\begin{array}{c}\text { estimated } \\
\text { body length } \\
(\mathrm{cm})\end{array}$ & skull shape & $\begin{array}{c}\text { external } \\
\text { nares } \\
\text { facing } \\
\end{array}$ & orbits facing & $\begin{array}{c}\text { occipital } \\
\text { condyle facing }\end{array}$ & limb posture & habitat \\
\hline Edentosuchus & $3.5-5$ & $30-40$ & oreinirostral & anteriorly & $\begin{array}{l}\text { dorsolaterally } \\
\text { laterally, }\end{array}$ & $?$ & $?$ & terrestrial \\
\hline Kayenta form & 6 & $40-50$ & oreinirostral & anteriorly & $\begin{array}{c}\text { anterolaterally } \\
\text { laterally, }\end{array}$ & posteroventrally & $?$ & terrestrial \\
\hline $\begin{array}{l}\text { Araripesuchus tsan. } \\
\text { Araripesuchus }\end{array}$ & 9.1 & $\sim 70$ & oreinirostral & anteriorly & $\begin{array}{l}\text { anterolaterally } \\
\text { laterally, }\end{array}$ & posteriorly & erect & terrestrial \\
\hline buitrer. & 13 & $80-90$ & oreinirostral & anteriorly & $\begin{array}{c}\text { anterolaterally } \\
\text { laterally, }\end{array}$ & $?$ & erect & terrestrial \\
\hline Araripesuchus gom. & 11.8 & 64 & oreinirostral & anteriorly & anterolaterally & posteriorly & erect & terrestrial \\
\hline Araripesuchus pat. & $\sim 7$ & $40-50$ & oreinirostral & anteriorly & $\begin{array}{c}\text { laterally, dorsolaterally } \\
\text { laterally, }\end{array}$ & posteriorly & erect & terrestrial \\
\hline Araripesuchus weg. & 12.73 & 81 & oreinirostral & anteriorly & $\begin{array}{c}\text { anterolaterally } \\
\text { laterally, }\end{array}$ & posteroventrally & erect & terrestrial \\
\hline Libycosuchus & 18 & 130 & oreinirostral & anteriorly & $\begin{array}{l}\text { anterolaterally } \\
\text { laterally, }\end{array}$ & posteroventrally & $?$ & ?terrestrial \\
\hline Uruguaysuchus & 13 & $80-90$ & oreinirostral & anteriorly & $\begin{array}{l}\text { anterolaterally } \\
\text { laterally, }\end{array}$ & posteriorly & $?$ & terrestrial \\
\hline Simosuchus & 12.7 & $60-65$ & platyrostral & anteriorly & $\begin{array}{l}\text { anterolaterally } \\
\text { laterally, }\end{array}$ & posteroventrally & semi-erect & terrestrial \\
\hline Malawisuchus & 7.6 & 60 & oreinirostral & anteriorly & $\begin{array}{l}\text { anterolaterally } \\
\text { laterally, }\end{array}$ & posteroventrally & erect & terrestrial \\
\hline Candidodon & 7.8 & $50-70$ & $\begin{array}{l}\text { oreinirostral } \\
\text { oreinirostral }\end{array}$ & anteriorly & $\begin{array}{l}\text { anterolaterally } \\
\text { laterally, }\end{array}$ & $?$ & erect & terrestrial \\
\hline Adamantinasuchus & 6 & $40-50$ & $?$ & anteriorly & $\begin{array}{l}\text { anterolaterally } \\
\text { laterally, }\end{array}$ & $?$ & $?$ & terrestrial \\
\hline Yacarerani & 9.5 & $70-80$ & oreinirostral & anteriorly & $\begin{array}{l}\text { anterolaterally } \\
\text { laterally, }\end{array}$ & posteroventrally & $?$ & terrestrial \\
\hline Mariliasuchus & 10.2 & $70-80$ & oreinirostral & anteriorly & $\begin{array}{l}\text { anterolaterally } \\
\text { laterally, }\end{array}$ & posteroventrally & $?$ & terrestrial \\
\hline Notosuchus & 14.2 & $100-150$ & oreinirostral & anteriorly & $\begin{array}{l}\text { anterolaterally } \\
\text { ?laterally, }\end{array}$ & posteroventrally & erect & terrestrial \\
\hline $\begin{array}{l}\text { Chimaerasuchus } \\
\text { Armadillosuchus }\end{array}$ & $\begin{array}{c}\sim 12-15 \\
\sim 30\end{array}$ & $\begin{array}{c}\sim 100-150 \\
180-200\end{array}$ & $\begin{array}{l}\text { oreinirostral } \\
\text { platyrostral }\end{array}$ & $\begin{array}{l}\text { anteriorly } \\
\text { anteriorly }\end{array}$ & $\begin{array}{l}\text { anterolaterally } \\
\text { dorsolaterally }\end{array}$ & $\begin{array}{l}? \\
?\end{array}$ & $\begin{array}{l}\text { erect } \\
\quad ?\end{array}$ & $\begin{array}{l}\text { terrestrial } \\
\text { terrestrial }\end{array}$ \\
\hline
\end{tabular}




\begin{tabular}{|c|c|c|c|c|c|c|c|c|}
\hline Pakasuchus & 6.1 & 55 & oreinirostral & anteriorly & $\begin{array}{c}\text { laterally, } \\
\text { anterolaterally } \\
\text { laterally, }\end{array}$ & $?$ & erect & terrestrial \\
\hline Sphagesaurus & 17.5 & $120-140$ & oreinirostral & anteriorly & anterolaterally & $?$ & $?$ & $\begin{array}{c}\text { terrestrial } \\
\text { semi- }\end{array}$ \\
\hline Unasuchus & $\sim 5$ & $\sim 40$ & platyrostral & dorsally & $?$ & $?$ & $?$ & $\begin{array}{c}\text { aquatic } \\
\text { semi- }\end{array}$ \\
\hline Bernissartia & 12 & 91 & platyrostral & dorsally & dorsally, dorsolaterally & posteriorly & sprawling & $\begin{array}{l}\text { aquatic } \\
\text { semi- }\end{array}$ \\
\hline Hylaeochampsa & 15-17 & 140-150 & platyrostral & dorsally & dorsally, dorsolaterally & posteriorly & $?$ & $\begin{array}{l}\text { aquatic } \\
\text { semi- }\end{array}$ \\
\hline Iharkutosuchus & 11.1 & 80 & platyrostral & dorsally & dorsally, dorsolaterally & posteriorly & $?$ & $\begin{array}{l}\text { aquatic } \\
\text { semi- }\end{array}$ \\
\hline Acynodon & 18.6 & $130-140$ & platyrostral & dorsally & dorsally, dorsolaterally & posteriorly & sprawling & $\begin{array}{l}\text { aquatic } \\
\text { semi- }\end{array}$ \\
\hline Brachychampsa & 39.2 & $250-300$ & platyrostral & dorsally & dorsally, dorsolaterally & posteriorly & sprawling & $\begin{array}{l}\text { aquatic } \\
\text { semi- }\end{array}$ \\
\hline Albertochampsa & 21.0 & 160 & platyrostral & dorsally & dorsally, dorsolaterally & posteriorly & sprawling & $\begin{array}{l}\text { aquatic } \\
\text { semi- }\end{array}$ \\
\hline Stangerochampsa & 20.0 & 160 & platyrostral & dorsally & dorsally, dorsolaterally & posteriorly & sprawling & $\begin{array}{l}\text { aquatic } \\
\text { semi- }\end{array}$ \\
\hline Allognatosuchus & $\sim 14$ & 〜120-150 & platyrostral & dorsally & dorsally, dorsolaterally & posteriorly & sprawling & $\begin{array}{l}\text { aquatic } \\
\text { semi- }\end{array}$ \\
\hline $\begin{array}{l}\text { Caiman latirostris } \\
\text { Osteolaemus } \\
\text { tetraspis }\end{array}$ & $\begin{array}{c}24 \\
16-20\end{array}$ & $\begin{array}{l}200-350 \\
140-190\end{array}$ & $\begin{array}{l}\text { platyrostral } \\
\text { platyrostral }\end{array}$ & $\begin{array}{l}\text { dorsally } \\
\text { dorsally }\end{array}$ & $\begin{array}{l}\text { dorsally, dorsolaterally } \\
\text { dorsally, dorsolaterally }\end{array}$ & $\begin{array}{l}\text { posteriorly } \\
\text { posteriorly }\end{array}$ & $\begin{array}{l}\text { sprawling } \\
\text { sprawling }\end{array}$ & $\begin{array}{l}\text { aquatic } \\
\text { semi- } \\
\text { aquatic }\end{array}$ \\
\hline
\end{tabular}

